\title{
Automating the planning of container loading for Atlas Copco: Coping with real-life stacking and stability constraints
}

Jonas Olsson, Torbjörn Larsson and Nils-Hassan Quttineh

The self-archived postprint version of this journal article is available at Linköping University Institutional Repository (DiVA):

http://urn.kb.se/resolve?urn=urn:nbn:se:liu:diva-161866

N.B.: When citing this work, cite the original publication.

Olsson, J., Larsson, T., Quttineh, N., (2019), Automating the planning of container loading for Atlas Copco: Coping with real-life stacking and stability constraints, European Journal of Operational Research, 280(3), 1018-1034. https://doi.org/10.1016/j.ejor.2019.07.057

Original publication available at:

https://doi.org/10.1016/j.ejor.2019.07.057

Copyright: Elsevier (24 months)

http://www.elsevier.com/

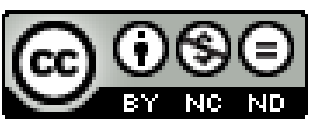




\title{
Automating the planning of container loading for Atlas Copco ${ }^{\text {th }}$ : coping with real-life stacking and stability constraints
}

\author{
Jonas Olsson, Torbjörn Larsson*, Nils-Hassan Quttineh \\ Department of Mathematics, Linköping University, SE-581 83 Linköping, Sweden
}

\begin{abstract}
The Atlas Copc园 distribution center in Allen, TX, supplies spare parts and consumables to mining and construction companies across the world. For some customers, packages are shipped in sea containers. Planning how to load the containers is difficult due to several factors: heterogeneity of the packages with respect to size, weight, stackability, positioning and orientation; the set of packages differs vastly between shipments; it is crucial to avoid cargo damage. Load plan quality is ultimately judged by shipping operators.

This container loading problem is thus rich with respect to practical considerations. These are posed by the operators and include cargo and container stability as well as stacking and positioning constraints. To avoid cargo damage, the stacking restrictions are modeled in detail. For solving the problem, we developed a two-level metaheuristic approach and implemented it in a decision support system. The upper level is a genetic algorithm which tunes the objective function for a lower level greedy-type constructive placement heuristic, to optimize the quality of the load plan obtained.

The decision support system shows load plans on the forklift laptops and has been used for over two years. Management has recognized benefits including reduction of labour usage, lead time, and cargo damage risk.
\end{abstract}

\footnotetext{
This project for Atlas Copco took place in 2015 and 2016. The company has since been split into Atlas Copco AB and Epiroc AB as of 2018, and the packing and container loading activities described in this paper are now part of the latter company.

* Corresponding author

Email addresses: jonasolovolsson@gmail.com (Jonas Olsson), torbjorn.larsson@liu.se (Torbjörn Larsson), nils-hassan.quttineh@liu.se (Nils-Hassan Quttineh)
} 
Keywords: logistics, packing, decision support systems, metaheuristics 2010 MSC: 90B06, 90B90, 90C59

\section{Introduction}

Atlas Copco is a Swedish company founded in 1873, which provides sustainable productivity solutions to customers in 180 countries across a wide range of industries. In 2016, the company had revenues of BEUR 10.7 and more than 42000 employees globally (Atlas Copco AB, 2017).

The company因 has a Distribution Center (DC) in Allen, TX, USA, which ships a large variety of spare parts and consumables to customers in drilling and rock excavation. Air-freight is the most common mode of transport for customers overseas, followed by sea container shipping which is slower but cheaper. The DC ships up to five containers per week. It is common that packages for multiple orders from a customer are accumulated over several days or weeks, and then shipped in a single $20 \mathrm{ft}$. or $40 \mathrm{ft}$. intermodal container. The shipping is made when the predicted volume utilization is high enough or when the customer requests the shipment to be made. It is crucial to avoid damage during transport by loading the container well and securing the cargo (using bands, straps and airbags), because the items shipped can have a high monetary value and also because cargo damage can ultimately cause costly downtime for the operations of the customers.

Prior to the implementation of our solution, the planning of the container loading was entirely manual. Due to significant variation in size, weight, stackability and orientation among packages, the planning often involved "pre-staging", that is, an attempt to physically perform the loading on the warehouse floor (see Figure 1). Its purpose was to ensure that all packages would fit and that the container would be "sufficiently full" (to warrant the fixed transportation cost), while at the same time avoiding apparent risks for cargo damage by ensuring cargo and container stability and by considering limitations on how packages can be stacked. The overall quality of a loading was ultimately judged by the DC shipping operators. Pre-staging was performed repeatedly by the operators, before giving customer service "go ahead" to order the container to be loaded. This time-consuming trial-anderror process caused excessive labour usage, delays in ordering containers, 
and consequently increased lead times for customers. The DC management understandably wanted to eliminate this process.

The complexity and diversity of shipments called for an optimization software. The ambition was to be able to use plans generated by the software to load containers without pre-staging, while maintaining or improving volume utilization and quality of loading (as defined by the DC operators). When loading, operators use forklifts equipped with small laptops, which could be used to show step-by-step instructions for placing each package (see Figure 2). Minor adjustments of the placements can of course be made. Further, running the software repeatedly as new orders arrive would allow customer service to know when to order a container.

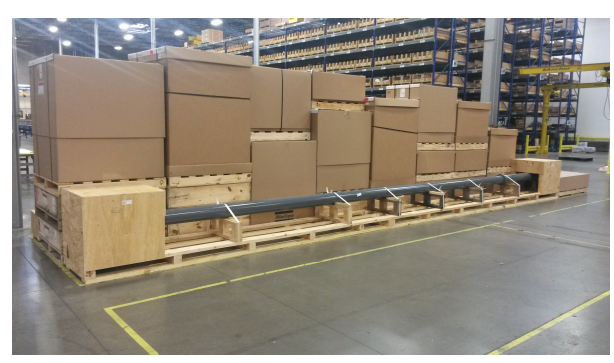

Figure 1: Pre-staging of a $40 \mathrm{ft}$. shipping Figure 2: Laptop in forklift that could be container (single column)

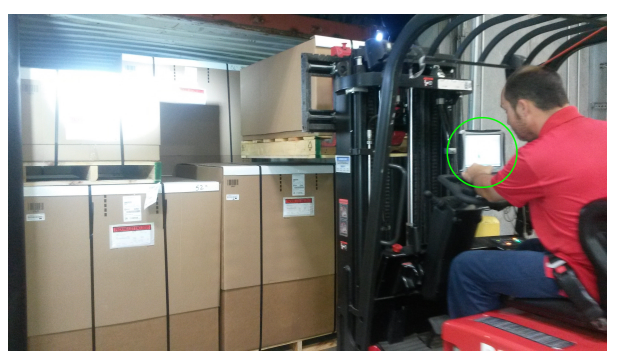

used to visualize a step-by-step load plan (single column)

A search for a container loading optimization software was conducted, starting from Douglas (2008), and there seemed to be plenty of attractive commercial options available. However, management determined that an "off-the-shelf" solution would require too much tailoring to fit the unique needs, and it was decided that an in-house software should be developed.

\subsection{Our problem in relation to state-of-the-art}

The state-of-the-art of the research on container loading is reviewed by Bortfeldt and Wäscher (2013) and Zhao et al. (2016). The former reference defines container loading as three-dimensional packing of boxes into rectangular containers, such that some objective function is optimized subject to applicable constraints. Hard constraints concerning geometry, such as boxes being inside the containers, and boxes not intersecting, are always included. Following these references, we also refer to cargo as boxes, except in contexts directly related to the Allen DC, when the term package is used. 
From these reviews we conclude that our container loading problem has several special characteristics. First, the packages to be loaded are given and expected to fit into a single container. Since the container selection is given, this is an output maximization problem (e.g., maximize volume utilization), in the typology by Wäscher et al. (2007). However, in such problem the container(s) are, by definition, not sufficient to accommodate all boxes, which is not the case in our setting. Further, in addition to volume utilization, we have multiple goals which are essential for the load plan quality, such as load stability. Second, seven out of the ten categories of constraints defined by Bortfeldt and Wäscher (2013) are included. No article in their review considers more than seven categories, and only $16 \%$ of the articles consider more than three constraints; hence, our problem is highly constrained. Third, our problem requires a much more realistic modelling of stacking constraints than what is common in the literature. Only $15 \%$ of the articles reviewed by Bortfeldt and Wäscher consider any stacking constraint. They also note (see Section 4.2.3) that the common approaches for modelling stacking strength, which are classification of boxes and maximum pressure, do not reflect that a box can withstand a higher pressure on the edges than in the center of its top surface. Fourth, our problem is heterogeneous in two respects (see Section 2.1). Since the number of sizes of packages is large compared to the total number of packages, our problem is strongly heterogeneous as defined by Wäscher et al. (2007). It is also heterogeneous in the sense that packages have ten attributes, in addition to size.

Both Bortfeldt and Wäscher (2013) and Zhao et al. (2016) remark that few articles adequately deal with issues relevant to real-life container loading. Zhao et al. conclude that "Only a small proportion of papers comprehensively address [real-world constraints], while most other papers focus on a few specific constraints. Even within those papers discussing real-world constraints, not all critical constraints are taken into consideration." Bortfeldt and Wäscher summarize that "In particular with respect to the inclusion of practically relevant constraints, research still has to be looked upon as being in its infancy." Regarding multiple goals, Bortfeldt and Wäscher (Section 5.2) remark that "only very few publications consider soft constraints. Nevertheless, violations of soft constraints can often be measured quantitatively. In this case, in addition to the underlying objective function, such measures can be taken as additional criteria for the evaluation of solutions 
(loading patterns), preparing the ground for multi-objective approaches." Finally, both reviews identify that a potential cause for neglecting issues relevant in real life is that the same data sets, which lack practical elements, frequently have been used to evaluate new approaches.

In the last few years, several papers have tackled real-life container loading problems, for example Costa and Captivo (2016), Paquay et al. (2016), Correcher et al. (2017), Toffolo et al. (2017), Moura and Bortfeldt (2017), and Alonso et al. (2019). However, even though data sets from companies have been used to evaluate performance of approaches, we note that evidence of successful implementations in day-to-day operations of companies is still lacking.

\subsection{Our work}

In the development of a software for the container loading problem at the DC, we looked for algorithmic approaches that could deal with the problem's full complexity, and especially approaches that involved at least four constraints, including stacking and stability. The approach by Techanitisawad and Tangwiwatwong (2004) was the most promising one found, since its overall design has an advantageous flexibility with respect to adding and altering both constraints and goals (cf. Figure 4 in the reference). Some useful concepts were also identified from other papers, see Section 2.5.

Our algorithm had to incorporate the essential judgments made by the DC shipping operators, via constraints and goals. None of this was however well-defined in the early stages of our work, which therefore included many experiments, for example pre-staging based on load plans generated by early versions of the software. This development methodology was possible thanks to the flexibility of the design by Techanitisawad and Tangwiwatwong (2004).

The paper proceeds as follows. In Section 2 we outline and analyse the container loading problem at the Allen DC, and review the literature that inspired the algorithmic approach used. In Section 3 the approach is given in more detail. Section 4 reports results for real-life instances. Section 5 gives some conclusions and reflections on benefits of using the developed software, and also discusses the applicability of our general solution concept to other highly constrained container loading problems. This paper is a short and streamlined version of the bachelor thesis by Olsson (2017). The major part 
of the development work at the DC took place in 2015 and 2016.

\section{Problem analysis}

The packing and shipping container loading process at the Allen DC is illustrated in Figure 3. At Levels 1 and 2, items are put into packages. At

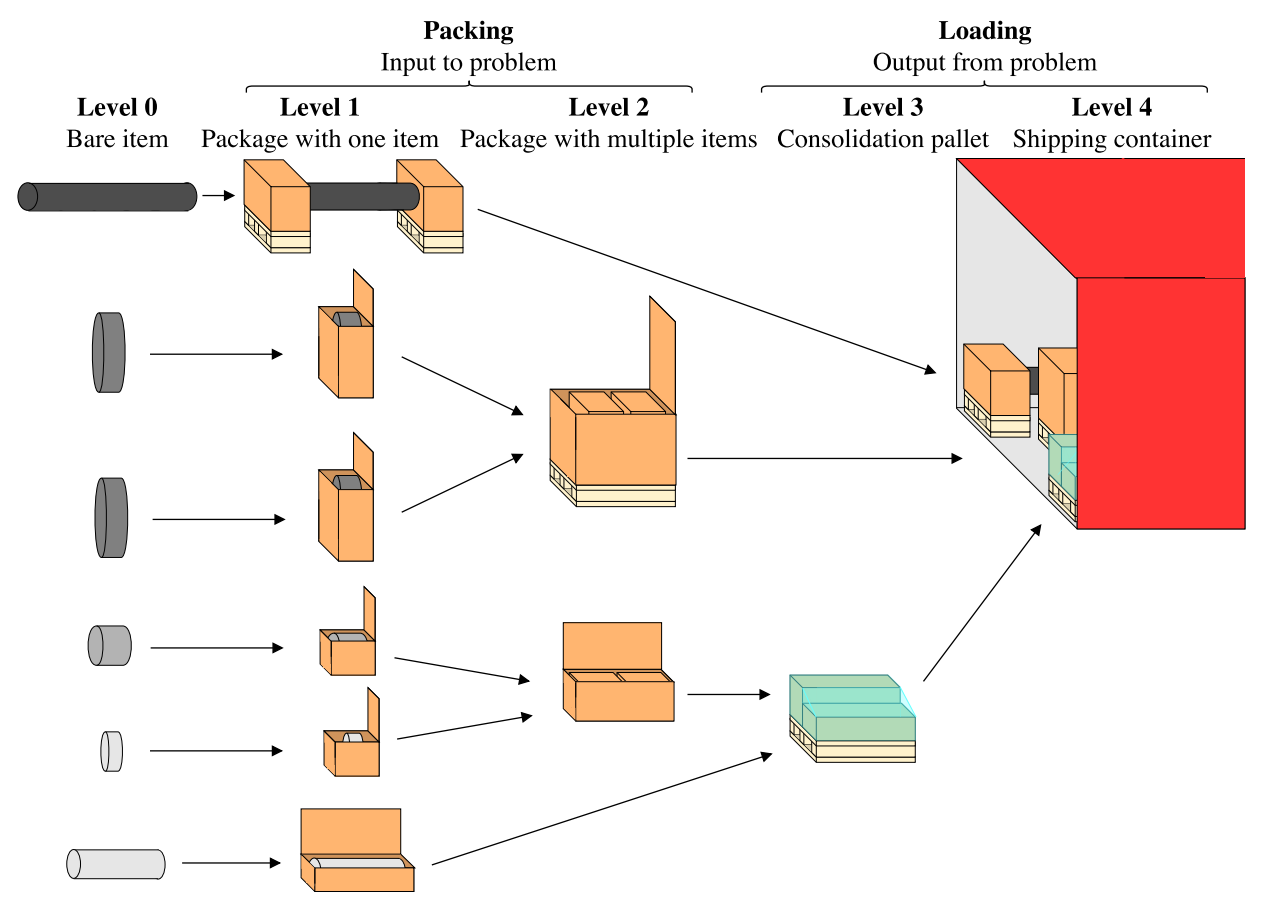

Figure 3: Packing and loading hierarchy (2 columns)

Level 3, smaller packages from Levels 1 and 2 are placed onto consolidation pallets, which are loaded into the container together with larger packages. These pallets prevent smaller packages from sliding and falling during transport. A load plan specifies Levels 3 and 4, that is, positions and orientations of smaller packages on consolidation pallets, and positions and orientations of larger packages and pallets within the container. We study the loading problem at Levels 3 and 4 only. The packages to be loaded are chosen such that they respect the weight limit of the shipping container and are expected to fit into the container. A primary loading goal is thus to fit all the packages into the container. (A leftover package may be shipped using air freight, or left behind and shipped in the next container.) 


\subsection{Characteristics of problem instances}

A problem instance consists of three components: packages to be loaded, a shipping container and available consolidation pallet types.

On Levels 1 and 2, there are 25 packaging options. Five of these are tailored and allow for customized packaging, creating an unlimited number of possible package sizes for Levels 3 and 4 . The most common packaging material is boxes of corrugated board. The packages have attributes that are inherited from their contents and packaging. Apart from size, the attributes are: 1) weight, 2) strength of packaging material if it provides support with edges or corners, 3 ) strength of packaging material if it provides support with only lid, 4) if a pallet is attached to the package, 5) whether the package has hazardous content, 6) if the pallet orientation differs from the agreed standard (pallet runners are parallel to the length of the package), and if so, how, 7) if the package must be placed on the floor, 8) if it is not feasible to stack a different package above, 9) if it is not feasible to stack a similar package above, and 10) reduced or increased stacking strength. The default value is false for attributes $6-9$, and 1.0 for attribute 10 . (The latter is $<1.0$ if fragile and $>1.0$ if extra strong.) Attributes 6-10 are called exceptions, while 5 and 7-10 are non-standard attributes.

Figure 4 illustrates the heterogeneity of the cargo. It shows weights, volumes and ratios between the longest and shortest package dimensions for 1948 packages from 50 shipments, with 11 to 88 packages per shipment. The packages were of 362 different sizes (due to $41 \%$ tailored packaging). The highest weight and volume were $3004 \mathrm{~kg}$ and $7.978 \mathrm{~m}^{3}$. The largest dimension ratio was 25.7. These shipments are studied in Section 4 .
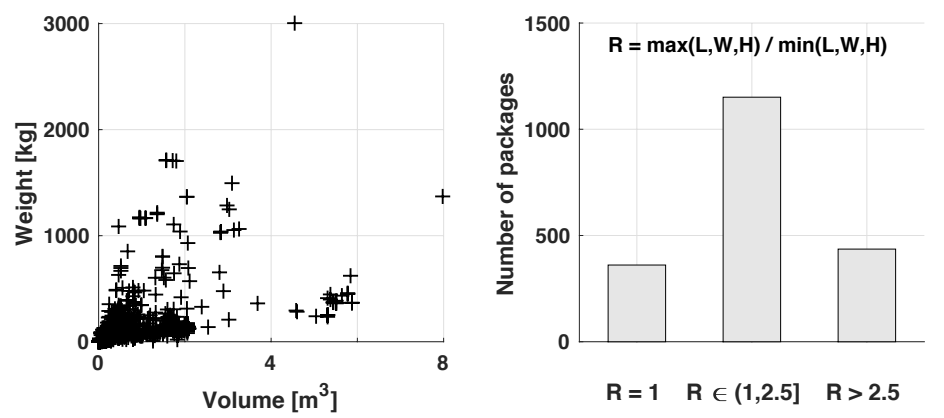

Figure 4: Characteristics for 1948 packages. Here, $R$ is the ratio between the largest and the smallest of a package's outer length (L), width (W) and height (H) (single column)

The choice of a $20 \mathrm{ft}$. or $40 \mathrm{ft}$. container may be limited by restrictions 
on the route to the customer's site, or by the lengths of the packages.

A consolidation pallet is an open box on a pallet or a pallet wrapped in plastic. The height can be adjusted within certain limits. When selecting consolidation pallets, the goal is to minimize their volume. Typically, zero to three consolidation pallets are used (of same or different types).

\subsection{Assessment of constraints}

Bortfeldt and Wäscher (2013) classify container loading constraints in ten categories. Further, there are hard constraints, which must be fulfilled, and soft constraints, which may be violated, at least within certain limits. In agreement with this, the DC shipping operators describe certain arrangements as unacceptable, and others as acceptable but not preferred. We model the former as hard constraints and the latter as soft constraints without limits, also called goals. These goals and the primary goal of fitting all packages into the container are summarized in Section 2.3. Seven of the categories of constraints are applicable to our problem: weight limits, weight distribution, orientation, complexity, stacking, positioning and stability.

\subsubsection{Weight limits, weight distribution, orientation and complexity}

The total weight of the boxes to be loaded into the shipping container is assumed to respect its weight limit.

Weight distribution can be captured in different ways. Lim et al. (2013), Pollaris et al. (2015), and Ramos et al. (2018) focus on truck axle weight. Bortfeldt and Wäscher (2013) note that several authors require the center of gravity being sufficiently close to the mid-point and the bottom of the container (see also Costa and Captivo, 2016). We adapt this as a goal for loading, referred to as container stability. Ramos et al. (2018) note that by not incorporating hard constraint based on truck axle weight, compliance with regulatory and technical requirements is not guaranteed.

Orientation constraints describe how boxes may be placed. We consider only orthogonal placements and cargo with a given vertical direction upon loading, since boxes are placed on pallets or marked "this way up". Hence, only two orientations are possible for all boxes, which is a hard constraint. Horizontal direction may matter for boxes with pallets attached when loading using a forklift. One orientation is preferred if the pallet runners are going in only one direction, and this is considered as a goal. 
Complexity constraints refer to technological and human limitations. For example, Bortfeldt and Wäscher (2013) stress that solutions must be visualized and easy to understand for the loading operators. Indeed, the need to visualize load plans using the forklift laptops is a complexity constraint in our case. Another is the goal to enable loading of pallets by forklifts, where the length of the forks pose a technological limitation. However, this could as well be classfied as a relative positioning contraint (see Section 2.2.2) as it concerns how boxes can be located relative to each other.

\subsubsection{Stacking and positioning}

Stacking constraints refer to how boxes can be placed on top of each other without causing damage. We consider these constraints as hard. With reference to Bortfeldt and Wäscher (2013), four factors have a significant impact upon how much weight or pressure a box may endure before bursting: 1) the strength of the packaging, which depends on the construction and the material of the box, 2) the content of the box, which can better withstand the load above if it is filled with non-fragile content, 3) the way of stacking, especially how many horizontal edges and corners (vertical edges) that are supporting a box above, since most of the strength of a box comes from the side walls (horizontal edges) and corners, and 4) transportation aspects, such as duration and the humidity throughout the route.

The DC operators recognize all four factors to be relevant in our problem. Initial experiments revealed that the third factor has a significant impact on stacking strength. For example, support from only one corner allows for a very restricted weight to be loaded above compared to the strength provided by support from two or more corners. Further, plenty of wall strength is still gained if the box above almost reaches the edges of the box below. Junqueira et al. (2012) and Alonso et al. (2014) limit stacking by a maximum weight per unit area of the top surface of the box below. Bischoff (2006) note that this approach to load bearing assumes that the same pressure can be supported anywhere on the top surface, while in practice the areas closer to the edges may withstand higher pressure than in the centre (in agreement with our empirical findings). Our stacking strength estimate is based on the Edge Crush Test (ECT), see Fibre Box Association (2008), which is a measure of a physical property of a corrugated board. The ECT value alone does not answer how much weight or pressure a box may endure before 
bursting, but it is used together with other parameters to estimate stacking strength.

Positioning constraints restrict the location of boxes within a container. We consider these constraints, except one, as hard. Absolute and relative positioning constraints refer to how single boxes can be located relative the container and relative other boxes, respectively. We face two absolute positioning constraints: "must be placed on the floor" (for example due to liquid content or heavy weight), and "not put boxes without pallets on the floor" (for example due to moisture on the floor). The latter constraint is soft and treated as a goal. There are two relative constraints: a box with hazardous content has to be easily accessible (no other box in front of it or above it), and the exceptions "not feasible to stack a different box above" and "not feasible to stack a similar box above" (which are due to some boxes not being rectangular, see the package in the top of Figure 3). The stackability attributes facilitate treating non-rectangular boxes as rectangular. The "different box" attribute should be true for any non-rectangular box. The "similar box" attribute is more limiting, as it implies that nothing can be stacked on top. (The package in the top of Figure 3 should have the former attribute as true, but possibly the latter as false.)

\subsubsection{Stability}

Stability constraints ensure that boxes do not slide or fall during transport, and there are two subcategories. Vertical stability constraints prevent boxes from falling down when the container is still, while horizontal stability constraints prevent boxes from shifting when the container is moving. Bortfeldt and Wäscher (2013) note that one way of dealing with the former is to require sufficient support of the base area of a box placed on top of other boxes. Ramos et al. (2016, Section 2.1) distinguish approaches found in the literature as: full base support, partial base support, or static mechanical equilibrium. As for horizontal stability, Bortfeldt and Wäscher (2013) note that one approach is to strive for support for the side surfaces of each box. For example, Costa and Captivo (2016) have, whenever possible, at least three sides touching the container walls or other boxes. In our case, placements resulting in interlocking of packages is preferred by the operators, while towers of boxes should be avoided. Vertical stability aspects pose hard constraints while horizontal stability aspects are goals. 


\subsection{Goals for loading}

There are four categories of goals, described by measurable objectives:

- Goal 1. Fit all packages into the container, by minimizing leftover packages with respect to: i) number, ii) volume, and iii) weight.

- Goal 2. Achieve horizontal cargo stability: i) by using consolidation pallets for smaller packages, and ii) by supporting the sides of packages and consolidation pallets.

- Goal 3. Achieve container stability (sufficiently good weight distribution) by placing center of gravity close to: i) lengthwise midpoint, ii) widthwise midpoint, and iii) floor.

- Goal 4. Avoid awkward placements by prioritizing to: i) place pallets in the same direction as their pallet runners, ii) enable loading of pallets by forklift, and iii) avoid packages without pallets on the floor.

Goal 1 is the primary concern. Goal 2 strives to reduce the risk of cargo damage during transport, without extensive use of securing materials. In exceptional cases (e.g., if the risk of cargo damage is too high) Goal 1 is sacrificed to better achieve Goal 2. Goal 3 strives for transportation safety; an unevenly distributed heavy load could adversely impact the stability of a truck transporting the container.

The consequence of not achieving Goal 4 is expending more labour on loading. If a package with pallet is not placed in the same direction as its pallet runners, the operator has to push and rotate the pallet using the forklift or by hand. A package with pallet is defined as unreachable if the forks cannot access at least $20 \%$ of its length, due to another package below blocking the truck. If the package is unreachable, the operator must adjust it by hand inside the container, or gain more leeway by stacking the packages outside the container first. Further, if a package without a pallet is to be placed on the floor, the operator may place some material below the package in order to protect it from moisture.

\subsection{A summary of our container loading problem}

Given a set of boxes which respects the container weight limit and is expected to fit into a single container, our loading problem can be given the following multi-objective description, inspired by the problem definition by Paquay et al. (2016, Section 2). 
maximize fulfilment of Goals 1-4

w.r.t. box placements that are feasible with respect to

hard geometry and orientation constraints

stacking constraints

positioning constraints

vertical stability constraints

Its solution is a load plan. The simultaneous fulfilment of Goals 1-4 is rated by a fitness function of a Genetic Algorithm (GA). Hence, a single-objective description of the problem is to maximize the load plan fitness.

\subsection{Derivation of solution approach}

Zhao et al. (2016) note that container loading is more often approached by heuristics than by mathematical models and exact algorithms. This is due to modelling challenges, and because derived models typically scale badly with problem size. Bortfeldt and Wäscher (2013) state that in the foreseeable future, only heuristics will be able to provide solutions of sufficient quality within acceptable computation times, for instances that are of relevant sizes and which include practical constraints. See Alonso et al. (2019) for recent progress in using mathematical models for large problems with practical constraints.

It is also noted by Zhao et al. (2016) that all heuristic approaches for container loading include a mechanism to place boxes, called a placement heuristic, which is often followed by an improvement heuristic, based on some metaheuristic principle. They distinguish between placement heuristics based on wall building, layer building, block building, and "boxes placed one at a time". Two common methods for placing boxes are to evaluate combinations of boxes and orientations together with either available spaces or placement positions, which are defined as points in three dimensions.

Zhao et al. (2016) distinguish methods based on how the box placement ordering is generated. In static sequencing, the ordering is determined before any placement is made and remains fixed throughout the loading procedure. In dynamic sequencing, the choice of which box to place next may depend upon previous placements; hence the ordering is known only afterwards. Both sequencing principles typically make use of some sort of scoring to generate the ordering, and the differences lie in which criteria that can be used for the scoring. Static criteria focus on characteristics of the boxes, 
while dynamic criteria can also include aspects such as characteristics of the remaining boxes and the available spaces for them.

In pursuit of a solution approach, we first classified our problem according to the criteria of Wäscher et al. (2007). The loading of consolidation pallets, at Level 3, is classified as a Single Bin Size Bin Packing Problem (SBSBPP) or as a Multiple Bin Size Bin Packing Problem (MBSBPP), while the loading of the shipping container, at Level 4, is a Single Knapsack Problem (SKP). Combining this classification with our analysis of constraints and goals led us to study four out of the 163 publications evaluated by Bortfeldt and Wäscher (2013) in more depth. The classifications (Wäscher et al. 2007) and constraint categories (Bortfeldt and Wäscher, 2013) of the problems considered in these four papers, which inspired our solution approach when the development project began (back in 2015), are in Table 1 compared with our problem. Here, RBPP stands for Residual Bin Packing Problem and Ext refers to an extension that also includes vehicle routing.

Table 1: Comparison of our problem and earlier works. The ten constraint categories are: 1. weight limit, 2. weight distribution, 3. loading priorities, 4. orientation, 5. stacking, 6. complete shipment, 7. allocation, 8. positioning, 9. stability, 10. pattern complexity. Here, a checkmark indicates that the constraint category is considered

\begin{tabular}{|c|c|c|c|c|c|c|c|c|}
\hline \multirow[t]{2}{*}{ Reference / Problem type } & \multicolumn{8}{|c|}{ Constraint categories } \\
\hline & 1 & 2 & 3 & $\begin{array}{lll}4 & 5 & 6\end{array}$ & 7 & 8 & 9 & 10 \\
\hline $\begin{array}{l}\text { Techanitisawad and } \\
\text { Tangwiwatwong (2004) } \\
\text { SKP, MBSBPP }\end{array}$ & & $\checkmark$ & & $\checkmark \checkmark$ & & & $\checkmark$ & \\
\hline $\begin{array}{l}\text { Lin et al. (2006) } \\
\text { SBSBPP, MBSBPP, RBPP }\end{array}$ & & & & $\checkmark \checkmark \checkmark$ & & $\checkmark$ & $\checkmark$ & \\
\hline $\begin{array}{l}\text { Wang et al. (2010) } \\
\text { SBSBPP Ext }\end{array}$ & $\checkmark$ & & & $\checkmark \checkmark \checkmark$ & $\checkmark$ & $\checkmark$ & $\checkmark$ & \\
\hline $\begin{array}{l}\text { Tarantilis et al. (2009) } \\
\text { SBSBPP Ext }\end{array}$ & $\checkmark$ & & & $\checkmark \checkmark \checkmark$ & $\checkmark$ & $\checkmark$ & $\checkmark$ & \\
\hline $\begin{array}{l}\text { Our work } \\
\text { SKP, SBSBPP, MBSBPP }\end{array}$ & $\checkmark$ & $\checkmark$ & & $\begin{array}{lc}\checkmark & \checkmark \\
& (1)\end{array}$ & (2) & $\begin{array}{l}\checkmark \\
(3)\end{array}$ & $\begin{array}{l}\checkmark \\
(4)\end{array}$ & $\checkmark$ \\
\hline
\end{tabular}

With reference to the problem description in Section 2.4, the constraint categories considered in our problem are taken into account as follows. The objective function includes soft constraints from categories $2,4,8,9$, and 10 . 
Constraints (i), (ii), (iii) and (iv) include hard constraints from categories 4 , 5,8 and 9 , respectively.

Techanitisawad and Tangwiwatwong (2004) present an integrated container selection and loading heuristic, with the selection made in an outer iteration and the loading in an inner iteration. The overall heuristic runs until all boxes are loaded. The loading procedure is a two-level heuristic guided by two scoring functions, the first of which selects a location and the second selects a box to place next. Each function includes nine components weighted together. The functions are tuned through a GA where the chromosome of an individual is an instance of the 18 weights. The nine components in the second function reflect characteristics of the box related to size, weight, feasible orientations, stackability and number of similar boxes. Each GA individual has a fixed box ordering, that is, static sequencing is used, and boxes are placed into containers one at a time. When no feasible placement remains, the GA fitness value is a function of the volume utilization and the container stability.

Lin et al. (2006) also use a GA, and propose an algorithm consisting of a grouping procedure and a packing procedure. Boxes are grouped based upon container capacities, dimensions of boxes, and unloading sequence. The grouping procedure determines the number of groups, assigns boxes to these groups, and selects potential containers for each group. The packing procedure loads the groups of boxes into the potential containers.

Wang et al. (2010) give two loading heuristics. In the first, every box is placed as close to the inner-bottom-left corner of the container as possible. In the second, every box is placed such that the total contact area, with either already placed boxes or container walls, is maximized. Tarantilis et al. (2009) propose six variants of loading heuristics, which are similar to those suggested by Wang et al. (2010) in that they encourage a packing structure with placements at corners and support from other boxes. They argue that with such a packing structure it is more likely to obtain feasible load plans.

Although our loading problem differs from those considered in the four above-mentioned papers, we borrow certain solution concepts from each of them. From Techanitisawad and Tangwiwatwong (2004) we adopt the twolevel concept of a scoring function for box placement sequencing and the use of a GA for tuning the scoring, since this concept is flexible with respect 
to the choice of constraints and goals. Unlike their work, we use dynamic sequencing. From Lin et al. (2006) we adopt the concept of grouping boxes, in our case with respect to package sizes. The design of components of our scoring function is inspired by evaluation criteria in the placement heuristics of Wang et al. (2010) and Tarantilis et al. (2009).

\section{Approach}

The loading problem considered is to determine placements of boxes within one or more containers. Our approach is generic, and a "box" may here refer to a single package or a consolidation pallet, while a "container" may refer to either a consolidation pallet or a shipping container.

A placement is a certain box placed in a certain way with respect to orientation, location (point) and container. A potential placement has not been made but considers a box not yet placed and an orientation at a point not yet occupied; it can be infeasible with respect to hard constraints. A performed placement is a feasible placement that is actually made. A complete load plan (or simply, load plan) refers to performed placements of all boxes, or a situation where there are remaining boxes but no feasible potential placements. A load plan that is not complete is said to be partial.

The next sections describe the overall structure of our algorithm and how it is applied in four stages, inspired by the loading process in practice. Thereafter we give more details about the components of the algorithm.

\subsection{Overall algorithm for container loading}

The proposed heuristic approach distinguishes between relevant properties of a complete load plan and properties of potential placements of boxes. The former are used to describe the agreed goals for loading (given by the operators), and the latter are designed with the purpose of steering the loading towards the agreed goals. For example, one may conceive that the center of gravity heightwise would turn out lower (a goal for loading) if priority is given to placing heavier boxes closer to the container floor (a property of potential placements) throughout the loading. Similarly, one may conceive that horizontal stability, that is, preventing boxes from sliding and falling (a goal for loading) would turn out better if support from other boxes or the container walls (a property of potential placements) is prioritised.

Our approach is a two-level metaheuristic method which consists of: 
(1) a constructive greedy-type placement heuristic,

(2) a procedure to evaluate a complete load plan, and

(3) a method for tuning priorities in the placement heuristic.

The first component is on the lower level, while the second and third components are on the upper level. The metaheuristic alternates between the two levels, as illustrated in Figure 5 .

The constructive heuristic respects all hard constraints and its potential placements are guided by a scoring function. The latter contains given criteria with temporarily fixed weights, the role of which is to prioritise between potential placements with different properties. The load plan evaluation assesses the fulfilment of the overall loading goals using a given rating function. The tuning aims to find values of the scoring weights that yield good placements with respect to the quality of the complete load plan, as measured by the rating function. The goals for loading are thus considered indirectly through the scoring function and its weights.

The multiple goals for loading and the heterogeneity of the problem instances make it impossible to find values of the scoring weights that yield satisfactory results for all instances. Hence, these weights need to be tuned for each instance, and we use a GA to do so, with a choice of weight values as a chromosome (genotype) in the population (cf. Techanitisawad and Tangwiwatwong, 2004). The placement heuristic decodes a chromosome into an individual (phenotype), which is a complete load plan, and the individual's fitness value is given by the load plan rating.

We let $W$ denote the population of chromosomes and let $W(i)$ be chromosome $i$. Further, $L(i)$ and $F(i)$ denote the complete load plan produced by chromosome $i$ and its fitness value, respectively, and $F$ denotes the set of all fitness values. Our two-level metaheuristic approach is shown to the left in Figure 5. At termination, the load plan with the highest rating found is returned. Both the placement heuristic and the load plan evaluation are deterministic, while the GA is stochastic. Our overall algorithm is selfadaptive in that it is capable of evaluating and changing its own behaviour whenever better performance may be possible.

A condition for the above strategy to work is that any loading decision that affects fulfilment of the loading goals, and thereby the load plan rating, can also be affected by the scoring function. This function must therefore include properties of potential placements that are interrelated to each loading 
goal, as shown to the right in Figure 5. We call this a steering mechanism.

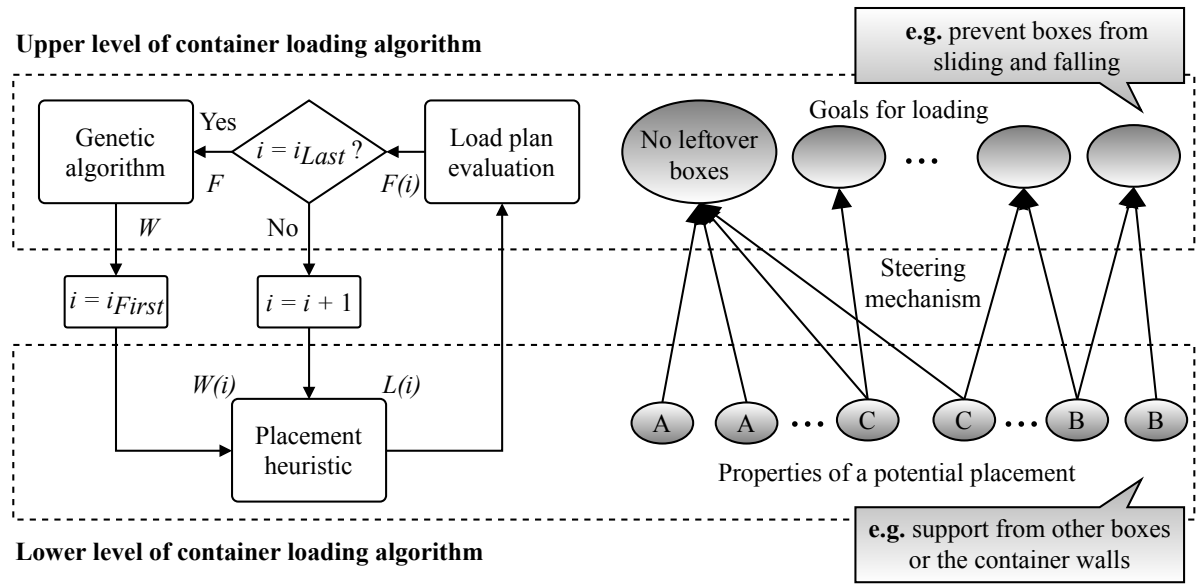

Figure 5: Illustration of upper and lower levels of the overall approach: schematic of the algorithm, and interrelations of loading goals and placement properties ( 2 columns)

In this figure "no leftover boxes" is emphasized since this goal is the most important. An interrelation between a property of a potential placement and a loading goal may be intended or occur as a side effect from a placement property introduced for another purpose. Three types of placement properties are distinguished, based on their purpose:

(A) to fit more boxes by improving the likelihood of feasible and "good" placements in subsequent iterations in the constructive heuristic,

(B) to achieve other desired goals for a complete load plan, and

(C) both (A) and (B).

Type (B) properties are more straightforward, whereas properties of type (A) are more common. An example of a type (A) property is "box ending at same height as adjacent box to the left", as it creates a flat base on which other boxes can be stacked. The property "support from other boxes or container walls" is an example of type (B). The property "heavier boxes closer to container floor" is of type (C), since limitations in stacking heavy boxes, due to the risk of damaging boxes below, makes it type (A), while the resulting lower center of gravity makes it type (B).

\subsection{Stages of loading}

The heterogeneity of the cargo demands for grouping of boxes (cf. Lin et al. 2006) and loading in stages (cf. Moura and Bortfeldt, 2017). The 
loading problem on Levels 3 and 4 in Figure 3 is divided into four stages, as shown in Figure 6. In Stages 1, 3 and 4, placements are performed, while

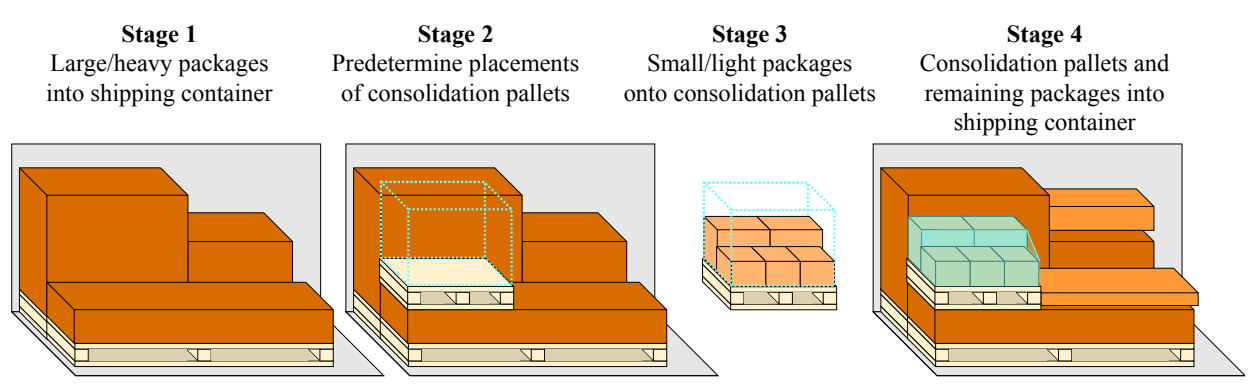

Figure 6: Stages of loading (2 columns)

Stage 2 prepares for Stages 3 and 4. Each stage proceeds with the best partial load plan found in the previous one. Each stage has its own rating function, and therefore no scoring weight values are transferred between stages. The two-level metaheuristic outlined in Section 3.1. to the left in Figure 5, is used in all four stages.

All boxes are assigned a priori to either Stages 1, 3 or 4, according to rules that are based on the packaging options used at the DC.

- Boxes in Stage 1. If at least one of the following conditions are true: i) box weight is greater than $36 \mathrm{~kg}$ ( $80 \mathrm{lbs}$ ), ii) maximum box dimension is greater than $1.5 \mathrm{~m}$, iii) the box has an attached pallet, and iv) the box needs to be placed on the container floor.

- Boxes in Stage 3. If all the following conditions hold: i) box weight is less than $36 \mathrm{~kg}$ (80 lbs), ii) maximum box dimension is less than $1.113 \mathrm{~m}$, iii) both box length and box width are less than $1.113 \mathrm{~m}$, iv) box length or box width is less than $0.732 \mathrm{~m}, \mathrm{v}$ ) box volume is less than $0.3 \mathrm{~m}^{3}$, vi) the box has no attached pallet, vii) the box needs not to be placed on the container floor, and viii) the box is not hazardous.

- Boxes in Stage 4. If not assigned to either Stage 1 or Stage 3.

In Stage 1, which is typically by far the most challenging, large and heavy boxes are loaded into the container. In Stage 2, the first step is to estimate the number of consolidation pallets needed, based on the volume of the packages in Stage 3. Each container point in the partial load plan obtained from Stage 1 is then evaluated, using a scoring function which focuses on the maximal possible height (due to the container ceiling or packages above) 
and weight (due to stacking strength of packages below) of the consolidation pallet, should it be placed at this point. This procedure yields the most suitable positions for the consolidation pallets, which in Stage 3 are loaded with the small and light packages. If no suitable positions were found in Stage 2, then consolidation pallets and Stage 3 are not used. In Stage 4, any consolidation pallets and any remaining packages are loaded into the container. Nothing may be stacked on top of a consolidation pallet.

The reason for not including consolidation pallets in Stage 1 is twofold. First, the loading of large, heavy packages should be prioritized. Second, the construction of the consolidation pallets depend of the outcome of Stage 1 .

The loading problems solved in the four stages are all of the same type as the overall problem stated in Section 2.4 and solved by the same algorithm, but with minor modifications for Stages 2 and 3. In Stage 2, consolidation pallets are considered as large boxes of variable height. Their maximal height and maximal weight capacity are goals, while their minimal height is a hard constraint. In Stage 3, a "container" to be loaded refers to a consolidation pallet, and if more than one such is used, then they are loaded simultaneously. Further, here the maximum possible weight for a consolidation pallet constitutes a hard constraint and is included in constraint (i).

Our four-stage procedure may be compared with the two-stage procedure by Moura and Bortfeldt (2017) and the stack building approaches used by Toffolo et al. (2017) and Correcher et al. (2017). Using the terminology of Zhao et al. (2016), our method is different in that we use block building only for small parts of the shipping container (with consolidation pallets as blocks), and "boxes placed one at a time" for the majority of the container, while the other authors use block building for the entire container.

There are several benefits of loading in stages. First, it simplified discussion of the software with DC personnel, since it mimics how the DC operators plan and perform loading manually. Second, it facilitates design with various goals and constraints for different stages of the loading, which helps to generate feasible solutions. Third, the time complexity is reduced by breaking up the overall problem into subproblems, since fewer potential placements are evaluated during each attempt to generate a load plan. 


\subsection{Placement heuristic}

Starting from the lower inner left corner, the algorithm constructs a load plan by placing boxes one at a time. They are put towards the right, towards the door, and upwards, without any specific priority among these directions. We consider potential placements as combinations of a box, an orientation, and a point in a container. When a box is loaded, its lower inner left corner is placed at the chosen container point.

Container points are created as boxes are loaded. We consider four types of points, shown in Figure 7 normal, projected, forward adjusted duplicate and backward adjusted duplicate. Normal points are generated on top of,

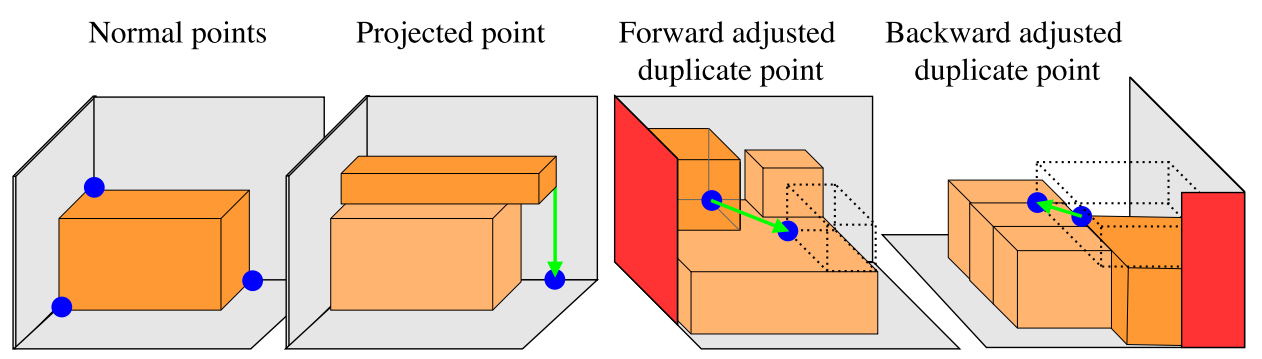

Figure 7: Types of points: normal, projected, forward adjusted duplicate and backward adjusted duplicate ( 2 columns)

in front of, and to the right of the last placed box. A normal point with no base support can be vertically projected to a point with base support. Normal and projected points, which are inspired by Martello et al. (2000) and Crainic et al. (2008), can be used for any box, while the adjusted points are generated uniquely for each remaining box. In order to gain stacking strength, existing points are duplicated and adjusted forward (lengthwise, widthwise, or diagonally) such that the remaining box ends exactly at the edges or corners of the box below. Analogously, in order to avoid intersection with the container walls or other boxes, existing points are duplicated and adjusted backward (lengthwise, widthwise, or diagonally) such that the remaining box ends i) exactly at the edges or corners of the box below, or ii) right before the walls of the container.

We use dynamic sequencing based on scoring of feasible potential placements. Each next placement is chosen in a greedy manner, that is, a maximal score is searched. The scoring incorporates relevant properties of feasible placements, such as consideration of unutilized volume, characteristics of remaining boxes, and to what extent the boxes have support. For a feasible 
potential placement, indexed by $k$, each property, indexed by $j$, is quantified by a scoring component, denoted $s_{j}(k)$, such that a better placement with respect to the property yields a higher value of the component.

There are three types of scoring components, with index sets $J_{1}, J_{2}$ and $J_{3}$. The first type is generic and designed so that $s_{j}(k) \in[0,1], j \in J_{1}$. The second type is designed so that $s_{j}(k) \in \mathbb{R}_{+}, j \in J_{2}$, and quantifies properties that are related to specific difficult loading aspects which may be necessary to prioritise in order to obtain a good load plan. Usually such properties are relevant only for a small subset of the potential placements. The third type is designed to enable its components $s_{j}(k) \in \mathbb{R}_{+}, j \in J_{3}$, to dominate the scoring entirely. In our case, $\left|J_{3}\right|=1$, and this component guarantees that consolidation pallets are placed at the points suggested by Stage 2 .

Each component $j \in J_{1} \cup J_{2}$ is given a weight $w_{j} \in[0, \bar{w}]$, to reflect its importance and thus the priority of the corresponding property when making a placement. To ensure scoring values of suitable magnitude, the two types of scoring weights are normalized separately. The score of feasible potential placement $k$ is

$$
S(k)=\frac{1}{\sum_{j \in J_{1}} w_{j}} \sum_{j \in J_{1}} w_{j} s_{j}(k)+\frac{1}{\bar{w}} \sum_{j \in J_{2}} w_{j} s_{j}(k)+\sum_{j \in J_{3}} s_{j}(k) .
$$

This design of the scoring function enables the second term to dominate the first, and the third term to dominate the second, if necessary to achieve the corresponding properties. Scoring components are further described in Section 3.3 .2 and Table 3 .

The placement heuristic is described in Algorithm 1. When assessing a potential placement, a specific box with a given orientation and a container point is considered. The score for a potential placement and the fulfilment of some of the hard constraints may depend on already performed placements; an example of the former is to what extent other boxes would be touching a box to be placed. Other aspects of a potential placement are independent of the placements already made, for example, if a box is placed on the container floor or not. Further, the evaluation of certain aspects require more intricate calculations, for example stacking strength constraints and scoring based upon unutilized volume. 


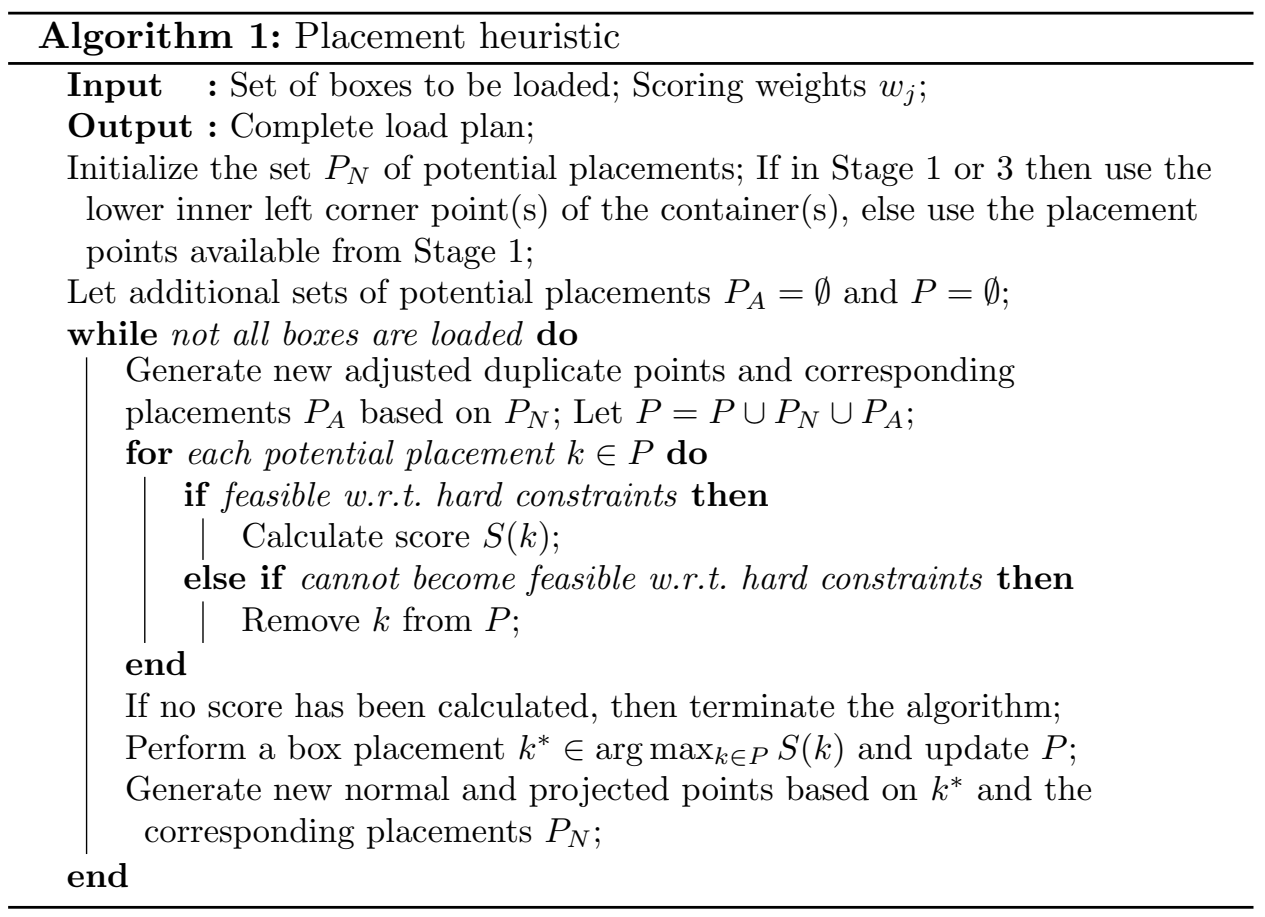

\subsubsection{Hard constraints}

We here give details on two of the hard constraints listed in Table 2. (Details on all hard constraints are given in Supplementary material, Section 3.)

Example: Our approach to stacking strength estimation has evolved from theory, experiments, crafting and testing. Feedback from the DC operators indicates that it works well in real-life situations. To calculate the maximum possible load (in lbs) on an area on top of a box, called MaxLoad, we apply the formula

$$
\text { MaxLoad }=\frac{5.87 \times E C T \times \sqrt{\text { Thickness } \times \text { Perimeter }}}{\text { EnvirFactor }},
$$

which is based on McKee et al. (1963). Here, the ECT value (in lbs per inch) is for the box to be stacked upon, Thickness is its wall thickness (in inches), Perimeter is the total length of its edges that support the box that is stacked, and EnvirFactor is an environmental factor (Fibre Box Association, 2008). Via ECT and Thickness, the strength of the packaging is captured. Typically ECT $\in[48,80]$ and Thickness $=0.25$ hold for packages at the DC. Depending on the content of a box (fragile or extra 
Table 2: Hard constraints in placement heuristic. Here, a checkmark indicates that the constraint is considered in the corresponding stage

\begin{tabular}{|c|c|c|c|c|c|c|}
\hline \multirow{2}{*}{\multicolumn{2}{|c|}{ No. Cat. }} & \multirow[t]{2}{*}{ Placement constraint } & \multicolumn{4}{|c|}{ Stage } \\
\hline & & & 1 & 2 & 3 & 4 \\
\hline 1 & Geom. & Box entirely inside container & $\checkmark$ & $\checkmark$ & $\checkmark$ & $\checkmark$ \\
\hline 2 & Geom. & Box not intersecting other boxes & $\checkmark$ & $\checkmark$ & $\checkmark$ & $\checkmark$ \\
\hline 3 & 4 & Box can have only two orientations & $\checkmark$ & $\checkmark$ & $\checkmark$ & $\checkmark$ \\
\hline 4 & 5 & Stacking strength of boxes below not exceeded & $\checkmark$ & $\checkmark$ & $\checkmark$ & $\checkmark$ \\
\hline 5 & 8 & $\begin{array}{l}\text { Box not on top of different box if the other } \\
\text { box is submitted as "not feasible to stack a } \\
\text { different package above" }\end{array}$ & $\checkmark$ & $\checkmark$ & $\checkmark$ & $\checkmark$ \\
\hline 6 & 8 & $\begin{array}{l}\text { Box not on top of similar box if the other box } \\
\text { is submitted as "not feasible to stack a similar } \\
\text { package above" }\end{array}$ & $\checkmark$ & $\checkmark$ & $\checkmark$ & $\checkmark$ \\
\hline 7 & 8 & $\begin{array}{l}\text { Box not above floor if submitted as "must be } \\
\text { placed on the floor" }\end{array}$ & $\checkmark$ & & & $\checkmark$ \\
\hline 8 & 8 & Box not in front of or above hazardous box & $\checkmark$ & $\checkmark$ & & $\checkmark$ \\
\hline 9 & 9 & Box receives sufficient base support & $\checkmark$ & $\checkmark$ & $\checkmark$ & $\checkmark$ \\
\hline 10 & $\mathrm{~N} / \mathrm{A}$ & Consolidation pallet minimum possible height & & $\checkmark$ & & \\
\hline \multirow[t]{2}{*}{11} & 1 & Container weight capacity not exceeded & & & $\checkmark$ & \\
\hline & & Total number of placement constraints & 9 & 9 & 8 & 9 \\
\hline
\end{tabular}

strong), the ECT can be adjusted. Despite its age, the McKee formula is still commonly used in commercial software (ibid.). We note that ECT is a measure for corrugated board. However, some packages at the DC are crates of wood that are stronger than corrugated board packages, and therefore given relatively high ECT values.

In the example in Figure 8, when evaluating the potential placement of box $B 3$ on top of $B 2$ there are two contact areas of interest, $A 1$ and $A 2$. If the sum of the weights of the boxes exceeds the MaxLoad value for an area, then the placement is not feasible and rejected. Boxes that are above and only partly within the boundaries of the area contribute a weight which is proportional to the size of the base area that lies within the boundaries. In the example, the weight on $A 2$ is that of $B 3$, while the weight on $A 1$ is that of $B 3$ plus a portion of the weight of $B 2$. We have three edges on $B 2$ supporting $B 3$, while there are only two edges on $B 1$ supporting $B 2$; hence, the support from $B 2$ to $B 3$ is better than that from $B 1$ to $B 2$.

The EnvirFactor is used to better capture weight distribution and support, as well as transportation aspects (e.g. duration and humidity). Our EnvirFactor was designed through experiments with packages at the 
DC. For example, different test cases were defined based on the number of edges/corners supporting the box above. For each test case, boxes where stacked accordingly until an experienced DC operator, who observed the impact on the box below, decided that the maximum load had been reached. This weight, which is the left-hand side of equation (2), was recorded. Since all factors in the numerator on the right-hand side of this equation were known, the denominator could be calculated. The range of the outcome over the different test cases was within a factor 3.4. Similar experiments led to our final EnvirFactor, which is a product of: 1) a factor [1.0,3.4] which concerns the number of edges/corners supporting the box above, 2) a factor $[0.33,1.00]$ which concerns if the box above may receive support from other boxes (overlap), 3) a factor 3.5 which captures all the remaining aspects. Hence the value of EnvirFactor falls within the range [1.2, 11.9]. Further, it is also included that much of the stacking strength from the walls and corners is received if the box above almost reaches the edges of the box below. This is the case in Figure 8 where strength from the rightmost wall of $B 1$ is gained as $B 2$ almost reaches the rightmost edge of $B 1$. To model this partial degree of support, a logistic function of the distance between the end of the box above and the edge of the box below is used. The aim is to capture the strength gained from walls and corners only when this distance is small enough. (For more details, see Supplementary material, Sections 2 and 3.)

Example: A box has sufficient base support to provide vertical stability if: i) all four corners have support below, or ii) two corners and $80 \%$ of base area have support below, or iii) $80 \%$ of base area has support below, the box is considered long with the largest dimension greater than $1.5 \mathrm{~m}$, and the box weight is less than $36 \mathrm{~kg}$ (80 lbs). We define a box as long if the ratio between its largest and second largest dimension is greater than 2.5.

\subsubsection{Scoring (including soft constraints)}

We give details on two of the scoring components listed in Table 3 . (Details on all scoring components are given in Supplementary material, Section 4.) 
Table 3: Placement properties quantified by scoring components in placement heuristic. Here, a checkmark indicates that the component is considered in the corresponding stage

\begin{tabular}{|c|c|c|c|c|c|c|c|}
\hline \multirow{2}{*}{\multicolumn{3}{|c|}{ No. Goal Type }} & \multirow[t]{2}{*}{ Placement scoring component } & \multicolumn{4}{|c|}{ Stage } \\
\hline & & & & 1 & 2 & 3 & 4 \\
\hline 1 & $1-3$ & $J_{1}$ & Box with higher weight closer to floor & $\checkmark$ & & $\checkmark$ & $\checkmark$ \\
\hline 2 & 1,2 & $J_{1}$ & Box closer to floor & $\checkmark$ & $\checkmark$ & $\checkmark$ & $\checkmark$ \\
\hline 3 & 1,2 & $J_{1}$ & $\begin{array}{l}\text { Box closer to intersection of inner wall and } \\
\text { ceiling lengthwise }\end{array}$ & $\checkmark$ & & & $\checkmark$ \\
\hline 4 & 1,2 & $J_{1}$ & $\begin{array}{l}\text { Box with higher stacking strength - if } \\
\text { would provide support with edges/corners } \\
\text { - closer to floor }\end{array}$ & $\checkmark$ & & $\checkmark$ & $\checkmark$ \\
\hline 5 & 1,2 & $J_{1}$ & $\begin{array}{l}\text { Box with higher stacking strength - if } \\
\text { would provide support with only lid, i.e. } \\
\text { no edges/corners - closer to floor }\end{array}$ & $\checkmark$ & & $\checkmark$ & $\checkmark$ \\
\hline 6 & 1,2 & $J_{1}$ & Utilization of space length higher & $\checkmark$ & $\checkmark$ & $\checkmark$ & $\checkmark$ \\
\hline 7 & 1,2 & $J_{1}$ & Utilization of space width higher & $\checkmark$ & $\checkmark$ & $\checkmark$ & $\checkmark$ \\
\hline 8 & 1,2 & $J_{1}$ & Utilization of space height higher & $\checkmark$ & $\checkmark$ & $\checkmark$ & $\checkmark$ \\
\hline 9 & 2 & $J_{1}$ & Support behind box & $\checkmark$ & $\checkmark$ & $\checkmark$ & $\checkmark$ \\
\hline 10 & 2 & $J_{1}$ & Support in front, left and right of box & $\checkmark$ & $\checkmark$ & $\checkmark$ & $\checkmark$ \\
\hline 11 & 1 & $J_{2}$ & Backward adjusted duplicate point is used & $\checkmark$ & $\checkmark$ & $\checkmark$ & $\checkmark$ \\
\hline 12 & 1 & $J_{2}$ & Forward adjusted duplicate point is used & $\checkmark$ & $\checkmark$ & $\checkmark$ & $\checkmark$ \\
\hline 13 & 1 & $J_{2}$ & $\begin{array}{l}\text { Box ending at same height as adjacent box } \\
\text { to the left }\end{array}$ & $\checkmark$ & & $\checkmark$ & $\checkmark$ \\
\hline 14 & 1 & $J_{2}$ & Box with same height as more other boxes & $\checkmark$ & & $\checkmark$ & $\checkmark$ \\
\hline 15 & 1 & $J_{2}$ & $\begin{array}{l}\text { Box on top of similar box with exception } \\
\text { "not feasible to stack a different package } \\
\text { above" }\end{array}$ & $\checkmark$ & & & $\checkmark$ \\
\hline 16 & 1 & $J_{2}$ & $\begin{array}{l}\text { Box with exception "must be placed on the } \\
\text { floor" }\end{array}$ & $\checkmark$ & & & \\
\hline 17 & 1 & $J_{2}$ & $\begin{array}{l}\text { Box considered to be long, with the largest } \\
\text { dimension greater than } 1.5 \mathrm{~m}\end{array}$ & $\checkmark$ & & & \\
\hline 18 & 1 & $J_{2}$ & $\begin{array}{l}\text { Hazardous box closer to intersection of } \\
\text { door and ceiling lengthwise, or box with } \\
\text { exception "not feasible to stack a similar/ } \\
\text { different package above" closer to ceiling }\end{array}$ & $\checkmark$ & & & $\checkmark$ \\
\hline 19 & 4 & $J_{2}$ & Box with pallet not rotated & $\checkmark$ & $\checkmark$ & & $\checkmark$ \\
\hline 20 & 4 & $J_{2}$ & Box without pallet not on floor & $\checkmark$ & & & $\checkmark$ \\
\hline 21 & 2 & $J_{1}$ & Consolidation pallet $\mathrm{p}$ & & $\checkmark$ & & \\
\hline 22 & 2 & $J_{1}$ & Consolidation pallet possible weight & & $\checkmark$ & & \\
\hline \multirow[t]{2}{*}{23} & 2 & $J_{3}$ & Consolidation pallet at point from Stage 2 & & & & $\checkmark$ \\
\hline & & & Total number of scoring components & 20 & 11 & 13 & 19 \\
\hline
\end{tabular}


Example: The scoring component used for prioritizing placing a heavy box close to the floor is the following.

$$
s_{1}(k)=\left(1-\frac{\text { height from floor }}{\text { container height }}\right) \times \frac{\text { weight of box }}{\text { max weight }}
$$

Here, "height from floor" is the vertical position of the center of gravity for the box if placed at the container point, and "max weight" is the weight of the heaviest box in the current stage.

Example: The scoring component below measures space utilization lengthwise, prioritizing leaving little space unused.

$$
s_{6}(k)=\text { Weighting }\left(\min \left\{1, \frac{\text { box length }}{\text { support length }}\right\}\right)
$$

Here, "support length" is the length of an area, on the floor or on top of other boxes, which can give support, and Weighting(.) is a nonlinear function (see Supplementary material, Section 2). The example in Figure 9 shows spaces for the points P2, P6 and P10. It is required that a space shall have full base support. The space for $\mathrm{P} 2$ is from the point to the container walls. Two potential spaces could be assigned to P6, and the one with the largest base area is chosen. The space for P10 coincides with one of P6's spaces.

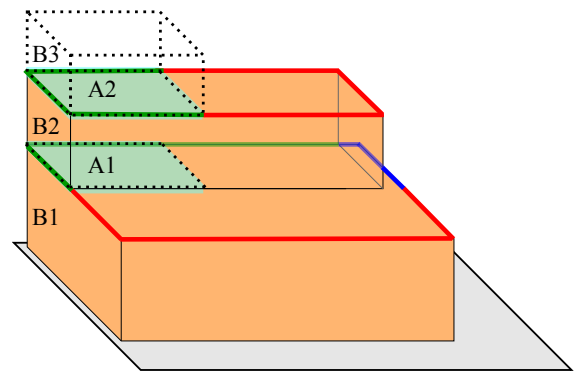

Figure 8: Evaluation of stacking strength: areas $A 1$ and $A 2$ for the potential placement of box $B 3$ on top of $B 2$ (single column)

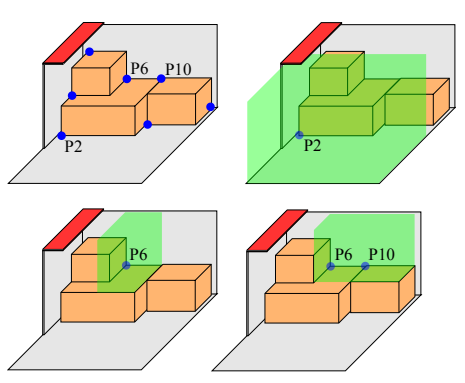

Figure 9: Evaluation of space utilization: spaces for the points $\mathrm{P} 2$, P6 and P10 (single column)

\subsection{Load plan evaluation}

Each feasible load plan is rated with respect to the goals and obtains a fitness value $F(i)$. The fitness component for goal $g$ is denoted $f_{g}(i)$. It is normalized such that $f_{g}(i) \in[0,1]$ and a higher goal fulfilment yields a higher value. Details about the fitness components used are given in Table 4 Since different goals are of different importance, they are assigned individual 
Table 4: Goals for loading quantified by fitness components in load plan evaluation. Here, a checkmark indicates that the component is considered in the corresponding stage

\begin{tabular}{|c|c|c|c|c|c|c|}
\hline \multicolumn{2}{|c|}{ No. Goal } & \multirow[t]{2}{*}{ Load plan fitness component } & \multicolumn{4}{|c|}{ Stage } \\
\hline & & & 1 & 2 & 3 & 4 \\
\hline 1 & 1 & Leftover boxes none & $\checkmark$ & $\checkmark$ & $\checkmark$ & $\checkmark$ \\
\hline 2 & 1 & Leftover boxes fewer & $\checkmark$ & $\checkmark$ & $\checkmark$ & \\
\hline 3 & 1 & Leftover boxes volume lower & $\checkmark$ & $\checkmark$ & $\checkmark$ & $\checkmark$ \\
\hline 4 & 1 & Leftover boxes weight lower & $\checkmark$ & $\checkmark$ & $\checkmark$ & $\checkmark$ \\
\hline 5 & 2 & Promising spaces for consolidation pallets & $\checkmark$ & & & \\
\hline 6 & 2 & Support behind boxes & $\checkmark$ & $\checkmark$ & $\checkmark$ & $\checkmark$ \\
\hline 7 & 2 & Support in front, left and right of boxes & $\checkmark$ & $\checkmark$ & $\checkmark$ & $\checkmark$ \\
\hline 8 & 2 & Utilization of floor space higher & $\checkmark$ & $\checkmark$ & $\checkmark$ & \\
\hline 9 & 2 & $\begin{array}{l}\text { Support for box not on floor - behind non-slim } \\
\text { box, and behind and in front of slim box }\end{array}$ & $\checkmark$ & $\checkmark$ & & $\checkmark$ \\
\hline 10 & 3 & Center of gravity lengthwise closer to center & $\checkmark$ & & & \\
\hline 11 & 3 & Center of gravity widthwise closer to center & $\checkmark$ & & & \\
\hline 12 & 3 & Center of gravity heightwise closer to floor & $\checkmark$ & & & \\
\hline 13 & 4 & Boxes with pallet not rotated & $\checkmark$ & $\checkmark$ & & \\
\hline 14 & 4 & Boxes with pallet reachable by forklift & $\checkmark$ & $\checkmark$ & & \\
\hline 15 & 4 & Boxes without pallet not on floor & $\checkmark$ & & & $\checkmark$ \\
\hline 16 & 2 & Consolidation pallets possible heiq & & $\checkmark$ & & \\
\hline 17 & 2 & Consolidation pallets possible weight & & $\checkmark$ & & \\
\hline 18 & 2 & Consolidation pallets closer to floor & & $\checkmark$ & & \\
\hline & & Total number of fitness components & 15 & 13 & 7 & 10 \\
\hline
\end{tabular}

fixed weights $c_{g} \in[1,30]$. The fitness value to be maximized is

$$
F(i)=1-\frac{1}{\sum_{g} c_{g}} \sum_{g} c_{g} \times\left(1-f_{g}(i)\right)^{2} .
$$

Clearly, this value is in the range $[0,1]$. (Details on all fitness components and their fixed weights are given in Supplementary material, Section 5.)

Example: The fitness component for the goal of minimizing the volume of leftover packages is

$$
f_{3}(i)=\frac{\text { loaded volume }}{\text { total shipment volume }} .
$$

Example: The fitness component for the goal of maximizing the support from behind for boxes is

$$
f_{6}(i)=\frac{\text { sum of support-from-behind-measure over all boxes }}{\text { loaded number of boxes }},
$$


where the support-from-behind-measure is the value of a logistic function of the contact area (with other boxes or the container walls behind the box) divided by the total area of the box's back side. (See Supplementary material, Sections 2 and 5.) The effect of the logistic function is to capture a threshold effect for what makes up sufficient support.

\subsection{Genetic Algorithm}

The GA procedure begins by randomly initializing the population $W$. In each generation, each chromosome in the population is assessed as shown to the left in Figure 5, resulting in fitness values $F$ for the population. The new population is produced as a combination of clones and children. The elitism concept is used for cloning. Children are created using the roulette wheel method for selecting parents, random crossover and mutation. Before applying the roulette wheel selection, each individual's fitness value is transformed via a nonlinear weighting function, such that $F(i)$ is replaced by Weighting $\left(F(i) / F\left(i^{*}\right)\right)$, where $i^{*}$ is the currently best individual with respect to fitness. (See Supplementary material, Section 2.) The reason for this is to amplify the probability for selecting individuals with high fitness values, relative to the currently best individual.

The GA parameter settings recommended by Techanitisawad and Tangwiwatwong (2004, Table 3) were used as a starting point, and experiments resulted in the following settings for our container loading algorithm. A chromosome consists of $\left|J_{1} \cup J_{2}\right|$ genes, each corresponding to a scoring weight $w_{j}$ which takes integer values between 0 and $\bar{w}=10,000$. The population size $|W|=3 \times\left|J_{1} \cup J_{2}\right|$, the cloning portion is $5 \%$, and the mutation probability for each gene in the chromosome of an offspring is $3 /\left|J_{1} \cup J_{2}\right|$. A mutated gene gets a random integer value in the range $[0, \bar{w}]$. In Stage 1 , $\left|J_{1} \cup J_{2}\right|=20,|W|=60$, and the gene mutation probability is $15 \%$.

\section{Results and analysis}

The proposed container loading metaheuristic has been successfully implemented as a software application at the DC. It was developed in Visual Basic for Applications (VBA) in Microsoft Access, and receives sizes and weights for packages from the enterprise software, while a separate application provides exceptions. Before running the software the user can see the calculated volume utilization. The software creates a load plan which 
suggests the placement of each package and consolidation pallet. The load plan is displayed on a laptop in a forklift upon loading.

We give computational results for 50 real problem instances. (All our problem data are given in Supplementary material, Section 8 and separate csv file.) The average number of packages is 39, average volume utilization (ratio between cargo and container volumes) is 54\%, and average number of unique package sizes is 20 . The instances and results are given in Table 5 . The computations were made on a Dell laptop with an Intel Core i5-2520M $2.50 \mathrm{GHz}$ CPU and 4 GB RAM.

Only Stage 1 results are reported, since it is by far the most crucial and challenging. On average $74.6 \%$ of the packages are loaded in Stage 1, their volume represent on average $96.4 \%$ of the total volume, and this stage includes the most fitness components (see Table 4). Since GA is stochastic, we made ten runs for each problem, with 200 GA generations in each run.

For each instance, we give the type of shipping container used (20 ft., $40 \mathrm{ft}$. or $40 \mathrm{ft}$. "high-cube"), total number of packages in all stages, ratio between cargo volume and container volume, number of unique package sizes, number of packages with non-standard attributes, proportion of Stage 1 packages, and proportion of Stage 1 package volume. The results presented are maximal number of generations before the GA found a solution with no leftover packages, range for generation when the best load plan was found, range for fitness value, and mean runtime in seconds per GA generation.

The primary goal is to fit all packages. If successful, the quality of the loading is of interest. Otherwise, the quality is of no interest. Therefore, the fitness values reported in the table are based only on components 5-15. In all runs on each problem all packages are loaded, except for two runs on each of Problems 19 and 29, and three runs on Problem 47. For 29 out of the 50 problems, all packages fit during the first GA generation in every run, and improvements were always made throughout the generations.

We conclude that the self-adaptiveness of our approach enables it to correctly identify which scoring components are crucial for the placement heuristic, to find a good overall load plan for each problem instance.

Figure 10 shows the GA fitness component values (see Table 4 ) and their ranges for the best load plan found in each of the ten runs on Problems 8, 12 and 19. (These instances are good representatives for the diversity in both problem characteristics and results.) The dot size represents the number of 
Table 5: Instances and Stage 1 results. Con. = container; Tot. = packages $(\#) ;$ Vol. = volume utilization (\%); Sizes = unique sizes (\#); Att. = non-standard attribute packages (\#); S1-P = Stage 1 packages (\%); S1-V = Stage 1 package volume (\%); Max. = max. generation with no leftover packages; Best = generation range for best found; Fitness $=$ range for fitness value $(\%)$; Time $=$ mean generation runtime (s). A '-' means $>200$

\begin{tabular}{|c|c|c|c|c|c|c|c|c|c|c|c|}
\hline Prob. & Con. & Tot. & Vol. & Sizes & Att. & S1-P & S1-V \| & Max. & Best & Fitness & Time \\
\hline 1 & 20 & 64 & 40 & 29 & 1 & 34.4 & 84.5 & 1 & 38185 & $99.4-99.6$ & 11 \\
\hline 2 & 20 & 20 & 43 & 14 & 9 & 80.0 & 98.5 & 1 & 48187 & $99.6-99.7$ & 5 \\
\hline 3 & 20 & 34 & 75 & 18 & 12 & 52.9 & 95.4 & 1 & 23199 & $99.8-99.9$ & 5 \\
\hline 4 & 40 & 30 & 47 & 12 & 3 & 96.7 & 99.5 & 1 & 46191 & $99.8-99.8$ & 18 \\
\hline 5 & 40 & 24 & 58 & 15 & 5 & 91.7 & 99.7 & 1 & 28194 & $99.8-99.8$ & 7 \\
\hline 6 & 40 & 38 & 47 & 18 & 2 & 97.4 & 99.7 & 4 & 44200 & $99.3-99.6$ & 32 \\
\hline 7 & $40 \mathrm{~h}$ & 26 & 47 & 19 & 0 & 92.3 & 99.7 & 1 & 13200 & $99.5-99.6$ & 10 \\
\hline 8 & 20 & 28 & 63 & 16 & 4 & 92.9 & 98.7 & 6 & 11195 & $99.6-99.7$ & 12 \\
\hline 9 & 40 & 40 & 46 & 19 & 3 & 95.0 & 99.9 & 1 & 133183 & $99.6-99.8$ & 29 \\
\hline 10 & 40 & 25 & 44 & 12 & 0 & 96.0 & 99.9 & 1 & 77185 & $99.7-99.8$ & 8 \\
\hline 11 & $40 \mathrm{~h}$ & 43 & 19 & 23 & 20 & 60.5 & 94.4 & 1 & 3167 & $97.6-98.8$ & 27 \\
\hline 12 & 20 & 33 & 59 & 17 & 3 & 51.5 & 97.3 & 14 & 33159 & $99.1-99.1$ & 4 \\
\hline 13 & 20 & 14 & 38 & 9 & 0 & 100.0 & 100.0 & 1 & 39180 & $99.1-99.3$ & 3 \\
\hline 14 & 40 & 34 & 47 & 19 & 13 & 97.1 & 99.9 & 1 & 33190 & $99.4-99.6$ & 25 \\
\hline 15 & 20 & 44 & 40 & 20 & 6 & 45.5 & 91.9 & 1 & $6 \quad 59$ & $99.6-99.6$ & 10 \\
\hline 16 & 20 & 46 & 71 & 30 & 7 & 76.1 & 97.2 & 196 & 39196 & $98.5-99.3$ & 28 \\
\hline 17 & 20 & 24 & 45 & 10 & 0 & 91.7 & 98.2 & 1 & $2 \quad 22$ & $99.9-99.9$ & 9 \\
\hline 18 & 40 & 11 & 21 & 9 & 7 & 81.8 & 99.6 & 1 & 1128 & $98.9-99.1$ & 1 \\
\hline 19 & 20 & 24 & 80 & 11 & 1 & 100.0 & 100.0 & - & 42180 & $99.6-99.6$ & 9 \\
\hline 20 & 20 & 49 & 43 & 25 & 4 & 46.9 & 93.6 & 6 & 37199 & $98.2-98.3$ & 11 \\
\hline 21 & 20 & 26 & 52 & 15 & 8 & 100.0 & 100.0 & 1 & $29 \quad 73$ & $99.6-99.8$ & 23 \\
\hline 22 & 40 & 19 & 59 & 12 & 2 & 94.7 & 99.9 & 1 & 17187 & $99.8-99.9$ & 6 \\
\hline 23 & 40 & 36 & 54 & 19 & 0 & 94.4 & 98.6 & 1 & 2163 & $99.8-99.8$ & 27 \\
\hline 24 & 20 & 27 & 63 & 11 & 0 & 100.0 & 100.0 & 1 & 1986 & $99.7-99.8$ & 28 \\
\hline 25 & 40 & 46 & 45 & 15 & 8 & 100.0 & 100.0 & 1 & $\begin{array}{ll}3 & 15\end{array}$ & $99.9-99.9$ & 119 \\
\hline 26 & 20 & 48 & 44 & 25 & 4 & 41.7 & 91.5 & 1 & 12152 & $99.4-99.6$ & 11 \\
\hline 27 & 20 & 63 & 61 & 29 & 4 & 52.4 & 91.3 & 7 & $6 \quad 52$ & $99.2-99.5$ & 40 \\
\hline 28 & 20 & 33 & 71 & 20 & 0 & 69.7 & 96.4 & 2 & 84164 & $99.8-99.8$ & 11 \\
\hline 29 & 20 & 38 & 72 & 13 & 3 & 100.0 & 100.0 & - & 55182 & $99.6-99.7$ & 42 \\
\hline 30 & 20 & 36 & 48 & 23 & 4 & 55.6 & 94.5 & 20 & 63198 & $99.1-99.5$ & 8 \\
\hline 31 & 20 & 16 & 45 & 14 & 0 & 100.0 & 100.0 & 1 & 8193 & $98.6-99.0$ & 6 \\
\hline 32 & 20 & 48 & 41 & 29 & 0 & 50.0 & 90.7 & 2 & 49102 & $99.0-99.4$ & 17 \\
\hline 33 & 40 & 27 & 46 & 17 & 2 & 88.9 & 99.4 & 1 & 10151 & $99.8-99.9$ & 12 \\
\hline 34 & 20 & 88 & 72 & 34 & 5 & 42.0 & 89.9 & 6 & $12 \quad 35$ & $99.4-99.8$ & 58 \\
\hline 35 & 20 & 13 & 84 & 10 & 0 & 92.3 & 99.9 & 3 & 1 & $99.8-99.8$ & 2 \\
\hline 36 & 20 & 70 & 57 & 20 & 1 & 40.0 & 92.3 & 1 & $31 \quad 55$ & $99.7-99.8$ & 32 \\
\hline 37 & 20 & 50 & 65 & 26 & 6 & 58.0 & 92.9 & 2 & $25 \quad 74$ & $99.7-99.8$ & 25 \\
\hline 38 & 20 & 69 & 75 & 23 & 3 & 50.7 & 93.1 & 20 & $8 \quad 60$ & $99.6-99.8$ & 45 \\
\hline 39 & 20 & 60 & 34 & 22 & 3 & 36.7 & 82.6 & 1 & 45105 & $99.8-99.9$ & 17 \\
\hline 40 & 40 & 33 & 52 & 19 & 2 & 90.9 & 99.1 & 1 & $27 \quad 82$ & $99.8-99.8$ & 18 \\
\hline 41 & 40 & 29 & 64 & 23 & 6 & 79.3 & 98.8 & 1 & 32167 & $99.8-99.9$ & 10 \\
\hline 42 & 20 & 35 & 28 & 21 & 7 & 71.4 & 93.5 & 1 & 2066 & $99.3-99.4$ & 23 \\
\hline 43 & 20 & 37 & 45 & 19 & 2 & 51.4 & 94.8 & 1 & 14197 & $99.6-99.7$ & 8 \\
\hline 44 & 20 & 42 & 75 & 24 & 2 & 73.8 & 96.3 & 14 & $17 \quad 78$ & $99.4-99.7$ & 23 \\
\hline 45 & 20 & 68 & 63 & 29 & 4 & 41.2 & 90.3 & 14 & 1480 & $99.1-99.5$ & 23 \\
\hline 46 & 20 & 62 & 63 & 24 & 5 & 51.6 & 94.9 & 103 & 20131 & $98.3-99.4$ & 22 \\
\hline 47 & 20 & 63 & 63 & 22 & 7 & 63.5 & 95.6 & - & 49188 & $97.9-98.9$ & 57 \\
\hline 48 & 40 & 54 & 56 & 23 & 8 & 90.7 & 98.5 & 18 & $2 \quad 27$ & $97.8-98.9$ & 125 \\
\hline 49 & $40 \mathrm{~h}$ & 44 & 68 & 24 & 1 & 79.5 & 98.2 & 5 & $8 \quad 59$ & $99.9-99.9$ & 33 \\
\hline 50 & 20 & 17 & 69 & 11 & 0 & 88.2 & 99.9 & 1 & 13109 & $99.8-99.8$ & 4 \\
\hline \multicolumn{2}{|l|}{ Mean } & 39 & 54 & 20 & 4 & 74.6 & 96.4 & & & & \\
\hline
\end{tabular}


similar values. Recalling that the highest possible fitness component value is 1 , it can be seen that the loading goals are fulfilled to a large extent and that the results are stable over the ten runs.

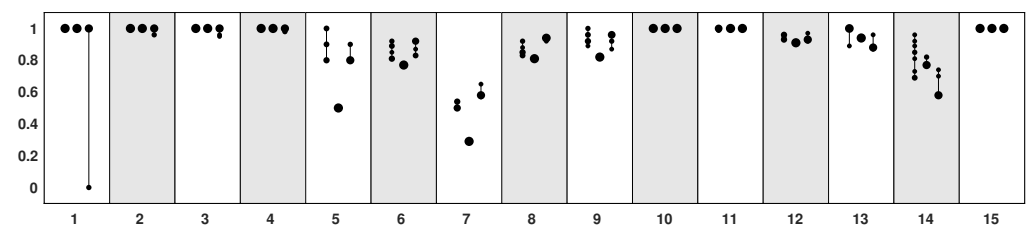

Figure 10: The values of the 15 fitness components in Stage 1 (see checkmarks in Table 4 ) for each of the Problems 8, 12 and 19, for the best load plans found in the ten runs (2 columns)

Figure 11 shows how the method successfully finds good fitness component values in the $50 \times 10=500$ runs. The high values of components 1-4 are due to several factors. First, packages are chosen so that they are expected to fit into a single container. Second, the fitness weights of these components $\left(c_{g}\right)$ are relatively large. Third, and most important, the tuning of the scoring weights and the dynamic sequencing enable the fitting of all packages; this is demonstrated in Section 4.3 .

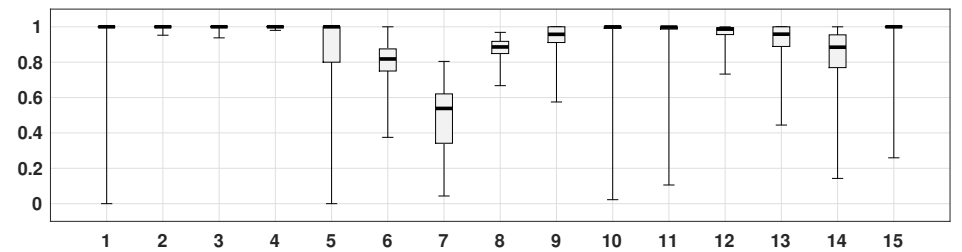

Figure 11: Distribution of Stage 1 fitness component values over the 500 runs (2 columns)

\subsection{Tuning of scoring weights}

We here study the values of the weights on the scoring components (see Table 3) and how they vary between instances. Figure 12 shows the distribution of the 20 scoring weights (in Stage 1) producing the best load plans over the ten runs for Problems 8, 12 and 19.

First, we note that for each problem instance the values of the scoring weights differ significantly. Second, the value of a certain scoring weight may vary significantly between problem instances. This confirms that the self-adaptiveness of our approach is indeed needed, to guide the placement heuristic to find good load plans. Third, in some cases a scoring weight 

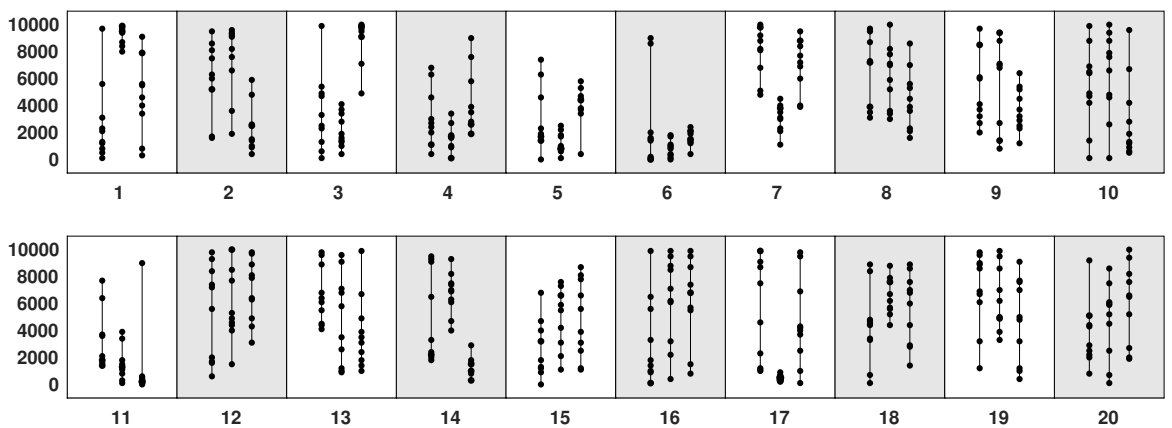

Figure 12: The values of the 20 scoring weights in Stage 1 (see checkmarks in Table 3) for the best load plan found in each of the ten runs on Problems 8, 12 and 19 (2 columns)

falls within a tight range, which indicates that the corresponding property of placements is important for finding good load plans, whereas others vary widely, indicating the opposite. (Scoring weight box plots for each problem instance, and an aggregated box plot illustrating the variation of the scoring weights over all $50 \times 10=500$ runs, are presented in Supplementary material, Section 6 , and they support the observations made above.)

\subsection{Load plan illustrations}

The purpose of the GA on the upper level of our overall approach (see Figure 5P is to tune the weights of the scoring function used in the lower level placement heuristic in order to optimize the outcome with respect to the quality of a complete load plan. We use the three earlier studied loading instances to illustrate that the GA is effective in tuning the scoring weights to values (see Figure 12) that are adequate for finding good load plans. The starting point for these illustrations is that we can by studying problem data foresee some necessary properties of good load plans. (In order to ease the practical loading of the packages, the order in which packages are printed in the visual load plan is often modified compared to the order obtained in the placement heuristic.)

For Problem 8 there is a possibility to nicely fit some large packages, widthwise and heightwise, in the back of the container. Such a load plan is found by the placement heuristic, see Figure 13, when the scoring weights $w_{7}$ and $w_{8}$ have been tuned to relatively high values. This means that the heuristic will prioritise potential placements with high widthwise and heightwise space utilization. This tight fit in the back of the container 
is further possible because packages are stacked with overlap, enabled by relatively high values of $w_{13}$, which makes the heuristic prioritise putting packages of the same height together.
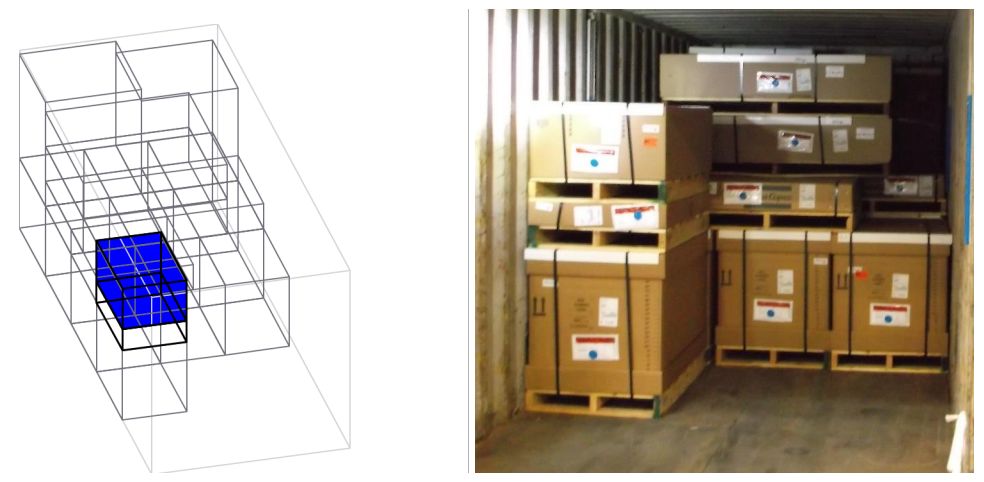

Figure 13: Laptop visualization of partial load plan for Problem 8 and actual loading, when 19 out of 28 packages have been placed. Package 19 is in blue (1.5 columns)

Problem 12 contains two long and heavy packages, $B 1$ and $B 2$, where $B 1$ is the heavier one. To fit all packages, it is necessary to stack packages $B 1$ and $B 2$ as shown in Figure 14. We note that scoring weights $w_{1}$ and $w_{2}$ are relatively high, indicating that it is beneficial to reward placements closer to the floor, especially for heavy packages. Given this, it is necessary for $w_{17}$ to be very low in order to prevent $B 1$ and $B 2$ from being placed too early, next to each other on the floor.

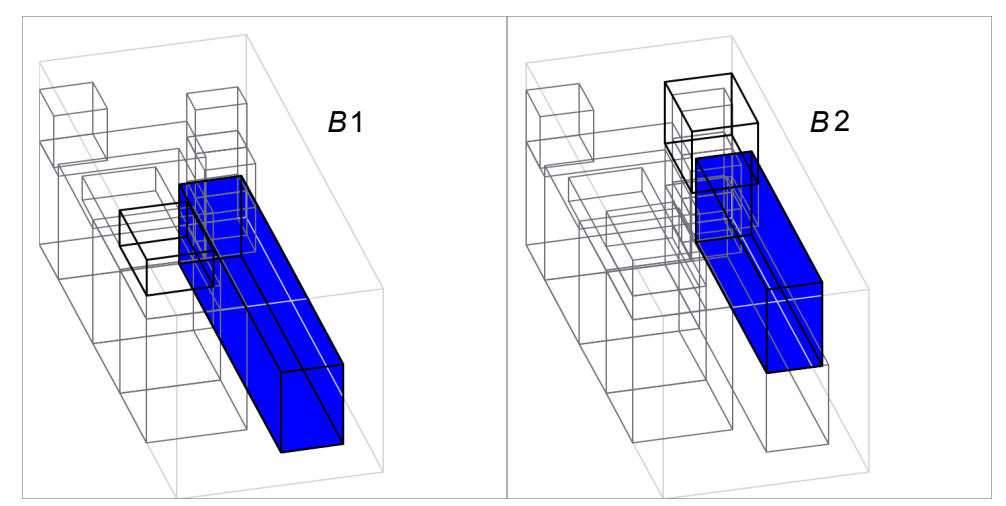

Figure 14: Laptop visualization of partial load plan for Problem 12, for placement of packages $B 1$ and $B 2$, which are packages 13 and 16 out of 33 (1.5 columns)

Problem 19 contains one huge and heavy package, requiring placement on the floor. It should clearly be placed first. Since all packages make up to as much as $80 \%$ volume utilization, it is critical to utilize the space on top of the 
huge package without exceeding stacking strength. For the heuristic to find a good load plan (see Figure 15), the scoring weight $w_{2}$ should be relatively low while $w_{3}$ should be distinctively high. Further, $w_{12}$ should be relatively high in order to prioritise the use of forward adjusted duplicate points, and thereby receive better support from below. (A step-by-step illustration of the load plan for Problem 19 is given in Supplementary material, Section 1.)

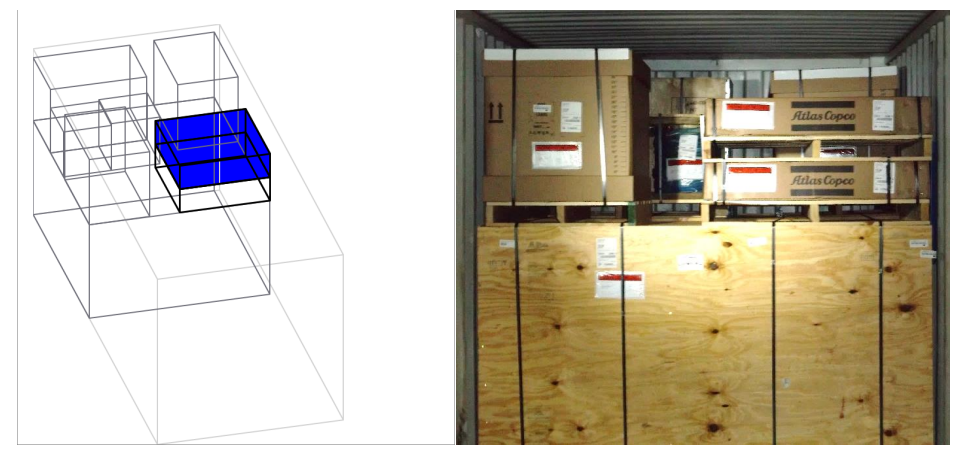

Figure 15: Partial load plan and actual loading for Problem 19, when 8 out of 24 packages have been placed ( 1.5 columns)

\subsection{Comparison with related versions of our algorithm}

We have demonstrated that our algorithm works well for the container loading problem at the DC. From a research point of view, it is of course also of interest to compare our method to previous work in the field. However, to the best of our knowledge, there is no other container loading problem with the same objectives and constraints. For example, no algorithm which considered that a box can withstand a higher pressure on the edges than in the center of its top surface was found. Hence, it is not possible to compare any other algorithm it its entirety to our full algorithm. Further, it is also of interest to verify that all components in our algorithm are indeed needed in order to achieve the successful results presented above.

To resolve these questions we designed an experiment with 21 related versions of our algorithm for Stage 1, and compared them to the algorithm implemented at the DC, referred to as version 22 . In the 21 related versions, three design choices were considered: i) the use of adjusted duplicate points; ii) the choice of components in the placement heuristic scoring function, and iii) tuning of the scoring weights versus using fixed weights. 
In the ten versions without adjusted duplicate points, only normal and projected points are used (see Figure 7). Four of the versions have scoring components based on Techanitisawad and Tangwiwatwong (2004) and for these versions their GA settings are used (ibid, Table 3). For all the other versions, the settings outlined in Section 3.5 are used. The scoring components referred to as "9+9" are identical to those in Techanitisawad and Tangwiwatwong (2004, Section 3.2.1). Here, each GA individual defines a static loading sequence. Eight of the versions have scoring components inspired by Crainic et al. (2008), referred to as "3HA" and "3AH". Here, static sequencing is used based on box properties (height, base area). Further, the scoring considers unutilized volumes, similarly to Crainic et al. The notation "20" refers to all our Stage 1 scoring components, while "18" excludes components for placing a box at an adjusted duplicate point. (Without adjusted duplicate points, scoring components 11 and 12 are redundant. Hence algorithm versions with all 20 scoring components but no adjusted duplicate points are irrelevant.) Finally, the notation "10" excludes all $J_{2}$ components.

For each related version with tuning of scoring weights, 10 runs were made for each of the 50 problem instances, with 200 GA generations in each run. For each of the 11 versions with fixed scoring weights, only one run was made for each instance since these versions are deterministic. Further, each fixed scoring weight is calculated as the average of the corresponding weight from the $50 \times 10$ runs with tuning. This means that the weights are fixed to fairly "good" values. The load plan evaluation and the hard constraints in the placement heuristic are the same for all 22 algorithm versions.

The algorithm versions are summarized in Table 6 together with the overall results in terms of fitting all packages and average fitness value. Fitness values are calculated only for runs where all packages fit. Bold numbers indicate the most successful versions, and the underlined numbers are the best. (Detailed results are given in Supplementary material, Section 7.)

The algorithm implemented at the DC (version 22) is best in terms of fitting all packages; it succeeds in 493 out of 500 runs. Close second comes version 21; it succeeds in 489 runs. Version 21 is actually slightly better than version 22 with respect to average fitness value, but the detailed results reveal that version 21 never succeeds in fitting all packages for Problem 19, whereas version 22 succeeds in 8 out of 10 runs. This is why version 22 is 
preferred.

Table 6: Stage 1 results for 20 versions in terms of fitting all packages and average fitness value (\%) for the runs where all packages fit. $\mathrm{T}=$ Tuning of the scoring weights (Yes/No); $\mathrm{C}=$ Components in the scoring function; $\mathrm{A}=$ Adjusted duplicate points used (Yes/No); Sum $=$ Sum of success rates of fitting all packages; Avg. = Average fitness values.

\begin{tabular}{c|ccccccccccc|ccccccccccc}
\hline Ver. & 1 & 2 & 3 & 4 & 5 & 6 & 7 & 8 & 9 & 10 & 11 & 12 & 13 & 14 & 15 & 16 & 17 & 18 & 19 & 20 & 21 & 22 \\
\hline $\mathrm{T}$ & $\mathrm{N}$ & $\mathrm{N}$ & $\mathrm{N}$ & $\mathrm{N}$ & $\mathrm{N}$ & $\mathrm{N}$ & $\mathrm{N}$ & $\mathrm{N}$ & $\mathrm{N}$ & $\mathrm{N}$ & $\mathrm{N}$ & $\mathrm{Y}$ & $\mathrm{Y}$ & $\mathrm{Y}$ & $\mathrm{Y}$ & $\mathrm{Y}$ & $\mathrm{Y}$ & $\mathrm{Y}$ & $\mathrm{Y}$ & $\mathrm{Y}$ & $\mathrm{Y}$ & $\mathrm{Y}$ \\
$\mathrm{C}$ & $3 \mathrm{HA}$ & $3 \mathrm{HA}$ & $3 \mathrm{AH}$ & $3 \mathrm{AH}$ & $9+9$ & $9+9$ & 10 & 10 & 18 & 18 & 20 & $3 \mathrm{HA}$ & $3 \mathrm{HA}$ & $3 \mathrm{AH}$ & $3 \mathrm{AH}$ & $9+9$ & $9+9$ & 10 & 10 & 18 & 18 & 20 \\
$\mathrm{~A}$ & $\mathrm{~N}$ & $\mathrm{Y}$ & $\mathrm{N}$ & $\mathrm{Y}$ & $\mathrm{N}$ & $\mathrm{Y}$ & $\mathrm{N}$ & $\mathrm{Y}$ & $\mathrm{N}$ & $\mathrm{Y}$ & $\mathrm{Y}$ & $\mathrm{N}$ & $\mathrm{Y}$ & $\mathrm{N}$ & $\mathrm{Y}$ & $\mathrm{N}$ & $\mathrm{Y}$ & $\mathrm{N}$ & $\mathrm{Y}$ & $\mathrm{N}$ & $\mathrm{Y}$ & $\mathrm{Y}$ \\
\hline \hline Sum & 20.0 & 20.0 & 28.0 & 28.0 & 27.0 & 24.0 & 21.0 & 21.0 & 13.0 & 14.0 & 15.0 & 29.6 & 28.5 & 36.2 & 37.2 & $\mathbf{4 5 . 3}$ & $\mathbf{4 6 . 6}$ & $\mathbf{4 6 . 5}$ & $\mathbf{4 6 . 9}$ & $\mathbf{4 8 . 3}$ & $\mathbf{4 8 . 9}$ & $\underline{\mathbf{4 9 . 3}}$ \\
Avg. & 98.8 & 98.8 & 98.6 & 98.5 & 98.7 & 98.7 & 98.5 & 98.6 & 98.9 & 98.9 & 98.9 & 99.0 & 99.0 & 98.9 & 98.8 & $\mathbf{9 9 . 4}$ & $\mathbf{9 9 . 4}$ & $\mathbf{9 9 . 5}$ & $\mathbf{9 9 . 5}$ & $\underline{\mathbf{9 9 . 6}} \underline{\mathbf{9 9 . 6}} \mathbf{9 9 . 5}$ \\
\hline
\end{tabular}

We conclude that the tuning of scoring weights is essential, and that the use of static sequencing is inadequate, for being able to fit all packages. Further, adjusted duplicate points are helpful in fitting all packages for difficult instances. In fact, those points were introduced, at a late stage in the development work at the DC, for exactly this purpose.

\section{Conclusions}

We have presented the outcome of a development project within an industrial company, with the purpose of automating their planning of container loading. The project has resulted in a software application which has been successfully implemented at the company's DC in Allen, TX. It has proven capable of generating satisfactory load plans within acceptable computation times, which may vary from 15 minutes to many hours, depending on the current planning situation. Further, the user can interrupt the optimization process whenever satisfied with the best available load plan. The software produces satisfactory solutions also for loadings deemed difficult by the operators. The software has been used for over two years at the DC.

Many different benefits of the software have been identified by various stakeholders: reductions in labour usage by eliminating pre-staging activities; reductions in lead time by eliminating delays in ordering containers; reductions in damage during transport by having DC operators focus more on securing cargo; improved safety by ensuring stability of containers; guaranteed loading quality even when less experienced operators execute the loading; improved customer service by providing visual load manifests to identify where packages are located within the container. Although consolidation pallets were occasionally used before, an unexpected outcome from 
the project was that they are now used on a regular basis.

The software is based on a two-level metaheuristic approach, which is motivated by the special characteristics of the studied container loading problem. All loading aspects are categorized as either hard constraints or goals. The core feature of our approach is that placements made by a constructive heuristic are steered towards an overall good load plan. All hard constraints are taken into consideration in the constructive heuristic. In each step of this heuristic, the potential placements that are feasible with respect to hard constraints are evaluated using a weighted scoring function, which prioritises between properties of the placements. The quality of a complete load plan is evaluated using a fixed ranking function, which describes the overall goals for loading. A genetic algorithm is used to adaptively adjust the weighted scoring function in order to obtain an overall good loading.

To meet real-life demands on limiting cargo damage we have paid special attention to stacking strength aspects by using the McKee formula and considering that most of the strength of a box comes from the side walls, and especially from corners (vertical edges).

As noted by Bortfeldt and Wäscher (2013) and Zhao et al. (2016), research on container loading problems dealing with several practical constraints is lacking. The design of our two-level container loading metaheuristic is very flexible with respect to the practical considerations that can be accommodated. Our solution approach should therefore also be well suited for other types of complex loading problems. We do however not expect it to be competitive for more standardized loading problems, since its design aims at dealing with complex settings.

Our two-level metaheuristic alternates between a greedy-type placement heuristic with dynamic ranking of alternatives using a local scoring function, and a tuning of parameters in this function in order to optimize the outcome with respect to a global rating function. The role of the tuning is to create local priorities that are consistent with the global objective. (We used a genetic algorithm to achieve this; other options are of course possible.) A necessary condition for this strategy to work is that any decision that affects the global objective can also be influenced by the local objective; our steering mechanism exemplifies this. From a research point of view, we consider this metaheuristic design to be of more general interest, in particular for optimization problems for which mathematical modelling is cumbersome or 
result in computationally intractable models.

\section{Acknowledgement}

Thomas Dahlgren and Ian Hale enabled the two summer internships for the first author at the DC in Allen, TX, which led to this paper. William Switzer provided incredible mentorship during these summers. Steven Vogel and Andrew Kirchner gave invaluable input, and feedback from actual loadings. Steve Bialas, Juan Cordova, Michael Moriarty, Matthew Scott, Tinto Skaria, and several others also made critical contributions.

We also wish to thank the anonymous reviewers for their constructive feedback which resulted in significant improvements of the paper. Finally, a special thanks to the editor for his patience throughout the long review process.

\section{References}

Alonso, M.T., Alvarez-Valdes, R., Iori, M., Parreño, F., 2019. Mathematical models for multi container loading problems with practical constraints. Computers \& Industrial Engineering 127, 722-733. doi:/10.1016/j.cie. 2018.11.012.

Alonso, M.T., Alvarez-Valdes, R., Tamarit, J.M., Parreno, F., 2014. A reactive grasp algorithm for the container loading problem with loadbearing constraints. European Journal of Industrial Engineering 8, 669694. doi:/10.1504/EJIE.2014.065732.

Atlas Copco AB, 2017. Atlas Copco annual report 2016. URL: /www.atlascopcogroup.com/investor-relations/financialreports-presentations/financial-publications

Bischoff, E.E., 2006. Three-dimensional packing of items with limited load bearing strength. European Journal of Operational Research 168, 952966. doi:/10.1016/j.ejor.2004.04.037.

Bortfeldt, A., Wäscher, G., 2013. Constraints in container loading a stateof-the-art review. European Journal of Operational Research 229, 1-20. doi:/10.1016/j.ejor.2012.12.006. 
Correcher, J., Alonso, M., Parreño, F., Alvarez-Valdes, R., 2017. Solving a large multicontainer loading problem in the car manufacturing industry. Computers \& Operations Research 82, 139 - 152. doi:/https://doi. org $/ 10.1016 /$ j.cor.2017.01.012

Costa, M.d.G., Captivo, M.E., 2016. Weight distribution in container loading: a case study. International Transactions in Operational Research 23, 239-263. doi:/10.1111/itor.12145.

Crainic, T., Perboli, G., Tadei, R., 2008. Extreme point-based heuristics for three-dimensional bin packing. INFORMS Journal on Computing 20, 368-384. doi:/10.1287/ijoc.1070.0250.

Douglas, M., 2008. Solving the cube. URL:/www.inboundlogistics.com/ cms/article/solving-the-cube.

Fibre Box Association, 2008. Edge crush test. URL: /www.fibrebox. myshopify.com/products/ect-application-and-reference-guide1 .

Junqueira, L., Morabito, R., Yamashita, D.S., 2012. Three-dimensional container loading models with cargo stability and load bearing constraints. Computers and Operations Research 39, 74-85. doi:/10.1016/j.cor. 2010.07.017.

Lim, A., Ma, H., Qiu, C., Zhu, W., 2013. The single container loading problem with axle weight constraints. International Journal of Production Economics 144, 358 - 369. doi:/https://doi.org/10.1016/j.ijpe. 2013.03.001.

Lin, J., Chang, C., Yang, J., 2006. A study of optimal system for multiple-constraint multiple-container packing problems. Advances in Applied Artificial Intelligence, proceedings 4031, 1200-1210. doi:/10.1007/ 11779568_127.

Martello, S., Pisinger, D., Vigo, D., 2000. The three-dimensional bin packing problem. Operations Research 48, 256-267. doi:/10.1287/opre.48.2. 256.12386 .

McKee, R.C., Gander, J.W., Wachuta, J.R., 1963. Compression strength formula for corrugated boxes. Paperboard Packaging 48, 149-159. 
Moura, A., Bortfeldt, A., 2017. A two-stage packing problem procedure. International Transactions in Operational Research 24, 43-58. doi:/10. 1111/itor.12251.

Olsson, J., 2017. Solving a highly constrained multi-level container loading problem from practice. URL: /http://urn.kb.se/resolve?urn=urn: nbn:se:liu:diva-134430.

Paquay, C., Schyns, M., Limbourg, S., 2016. A mixed integer programming formulation for the three-dimensional bin packing problem deriving from an air cargo application. International Transactions in Operational Research 23, 187-213. doi:/10.1111/itor.12111.

Pollaris, H., Braekers, K., Caris, A., Janssens, G.K., Limbourg, S., 2015. Vehicle routing problems with loading constraints: state-of-the-art and future directions. OR Spectrum 37, 297-330. doi:/10.1007/s00291-0140386-3.

Ramos, A.G., Oliveira, J.F., Gonçalves, J.F., Lopes, M.P., 2016. A container loading algorithm with static mechanical equilibrium stability constraints. Transportation Research Part B 91, 565-581. doi:/10.1016/j.trb.2016. 06.003

Ramos, A.G., Silva, E., Oliveira, J.F., 2018. A new load balance methodology for container loading problem in road transportation. European Journal of Operational Research 266, 1140-1152. doi:/10.1016/j.ejor. 2017.10 .050 .

Tarantilis, C.D., Zachariadis, E.E., Kiranoudis, C.T., 2009. A hybrid metaheuristic algorithm for the integrated vehicle routing and threedimensional container-loading problem. IEEE Transactions on Intelligent Transportation Systems 10, 255-271. doi:/10.1109/TITS.2009.2020187.

Techanitisawad, A., Tangwiwatwong, P., 2004. A GA-based heuristic for the interrelated container selection loading problems. Industrial Engineering and Management Systems 3, 22-37. URL:/http://citeseerx.ist.psu. edu/viewdoc/summary?doi=10.1.1.127.2898.

Toffolo, T.A.M., Esprit, E., Wauters, T., Vanden Berghe, G., 2017. A two-dimensional heuristic decomposition approach to a three-dimensional 
multiple container loading problem. European Journal of Operational Research 257, 526 - 538. doi:/https://doi.org/10.1016/j.ejor.2016. 07.033

Wang, L., Guo, S., Chen, S., Zhu, W., Lim, A., 2010. Two natural heuristics for 3D packing with practical loading constraints. PRICAI 2010: Trends in Artificial Intelligence: 11th Pacific Rim International Conference on Artificial Intelligence 6230, 256-267. doi:/10.1007/978-3-642-152467_25.

Wäscher, G., Hauner, H., Schumann, H., 2007. An improved typology of cutting and packing problems. European Journal of Operational Research 183, 1109-1130. doi:/10.1016/j.ejor.2005.12.047.

Zhao, X., Bennell, J., Bekta, T., Dowsland, K., 2016. A comparative review of $3 \mathrm{D}$ container loading algorithms. International Transactions in Operational Research 23, 287-320. doi:/10.1111/itor.12094. 
Automating the planning of container loading for Atlas Copco: coping with real-life stacking and stability constraints

\section{Supplementary material}

\section{Contents}

1. Some illustrations

2. Auxiliary functions

3. Placement heuristic hard constraints

4. Placement heuristic scoring components

5. Load plan evaluation fitness components

6. Tuning of the scoring weights

7. Results for algorithm versions

8. Comments on the problem data

The bachelor thesis "Solving a highly constrained multi-level container loading problem from practice" by Jonas Olsson provides a thorough background to the paper, and can be downloaded from the link below.

/http://liu.diva-portal.org/smash/get/diva2:1073644/FULLTEXT01.pdf 


\section{Some illustrations}

We give some pictures of packages and consolidation pallets from the DC in Allen, TX, and a step-by-step illustration of the load plan for Problem 19, as shown on the laptop in the forklift.

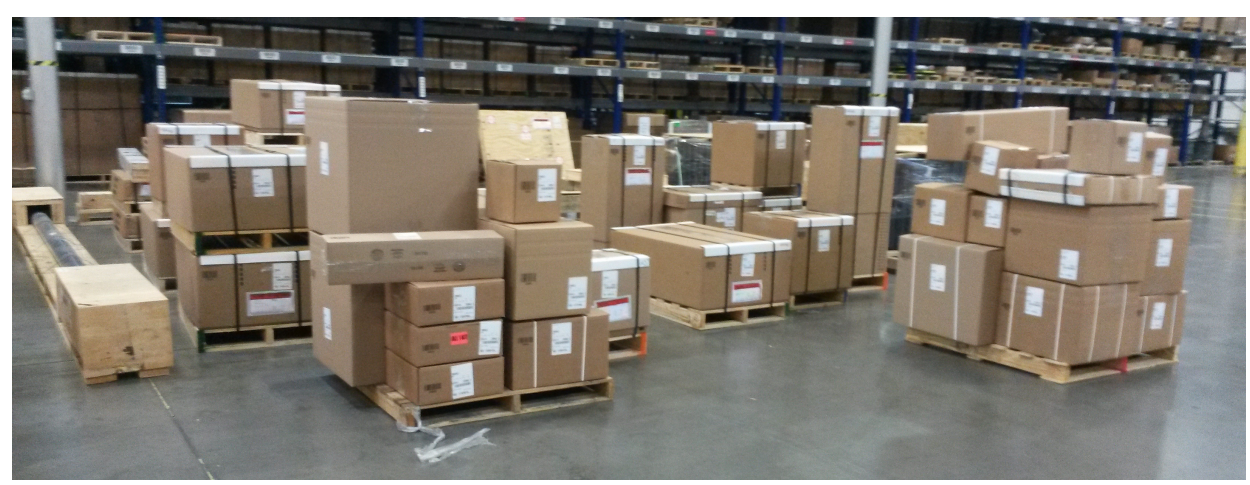

Figure 1: Packages of varying sizes and weights
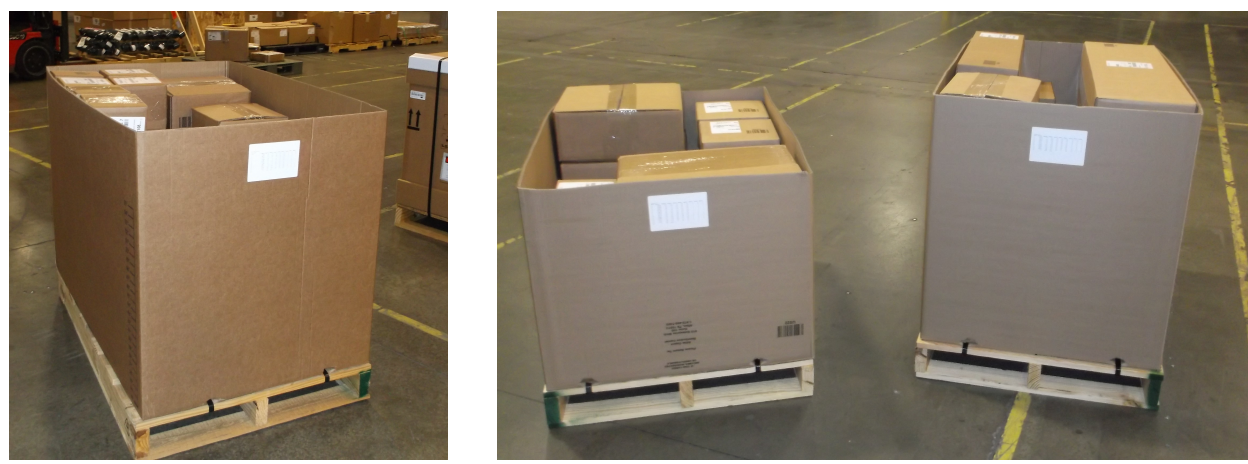

Figure 2: Examples of consolidation pallets 

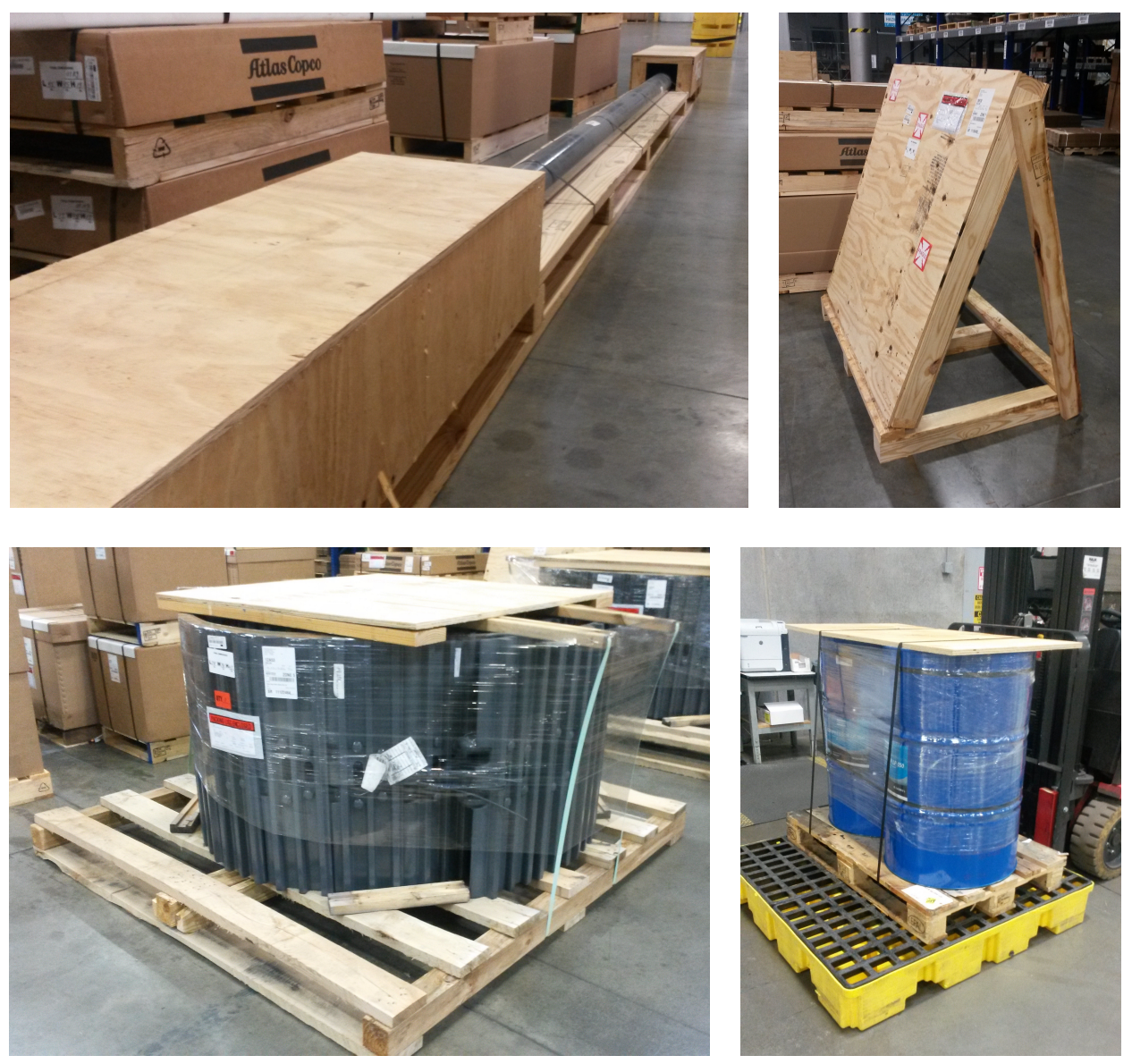

Figure 3: Upper left: a package which allows a similar package to be stacked above, but not a different package; upper right: a package (containing glass) which does not allow any package to be stacked above; lower left: a package that must be placed on the floor, due to its weight; lower right: a package that must be placed on the floor, due to fluid content which may leak out 


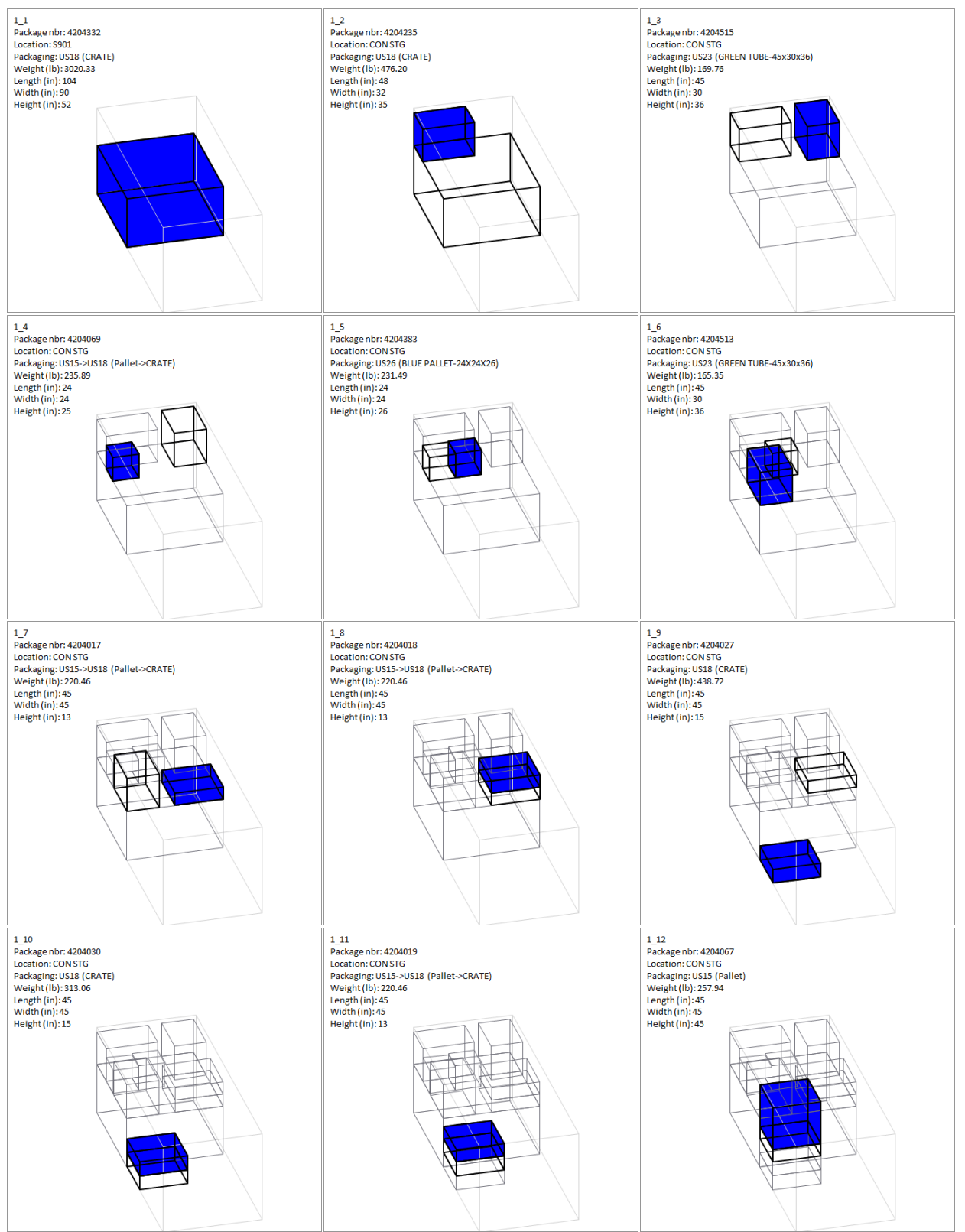

Figure 4: Load plan for Problem 19, steps 1-12, as shown to the operator on the forklift laptop 


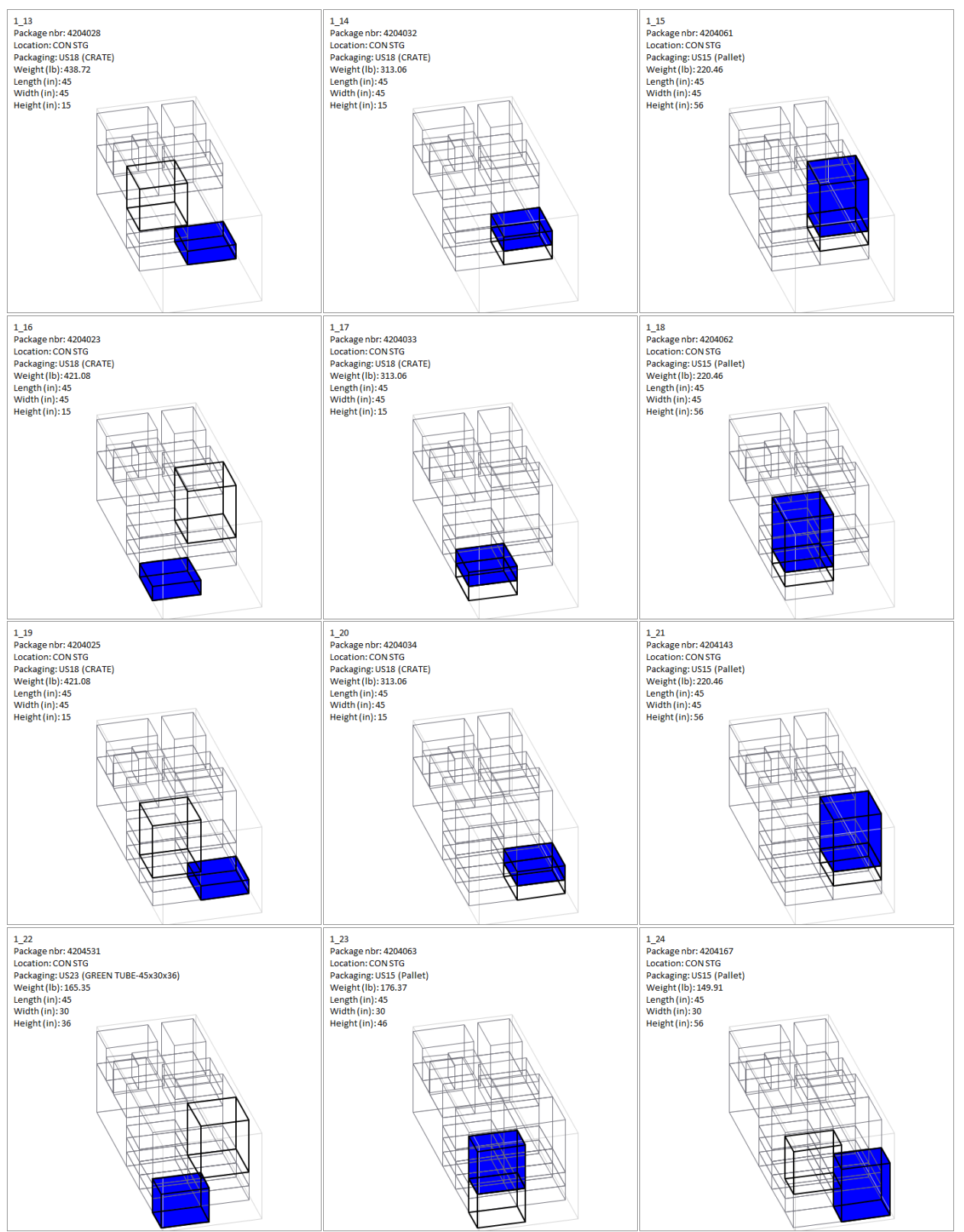

Figure 5: Load plan for Problem 19, steps 13-24, as shown to the operator on the forklift laptop 


\section{Auxiliary functions}

Here we specify auxiliary functions used in the calculations. For calculating threshold effects, the logistic (sigmoid) function

$$
\operatorname{Logistic}(x, m, s)=\frac{1}{1+e^{-s(x-m)}}
$$

is used, where $m$ and $s$ are midpoint and steepness parameters, respectively.

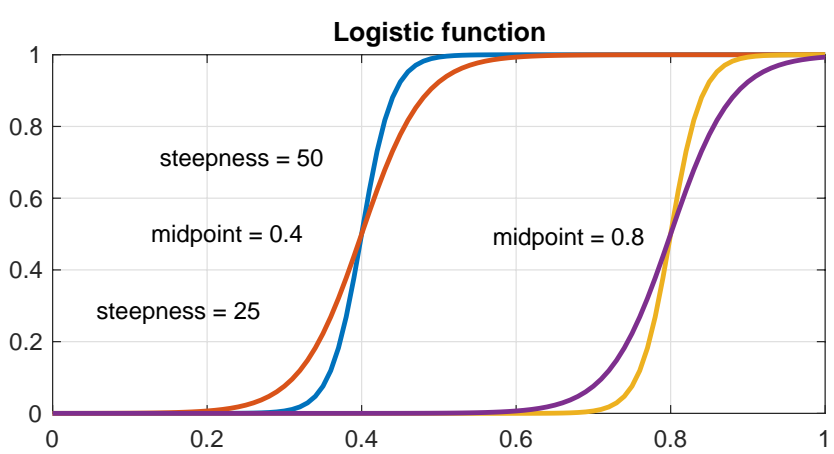

Figure 6: Examples of logistic functions

In order to further distinguish high values of a measure, the nonlinear weighting function

$$
\operatorname{Weighting}(x, f)=\frac{f}{f+(1-x)}
$$

is used, where $f$ is a parameter. For example, we use this function to amplify the probability for selecting individuals with high fitness values in the GA.

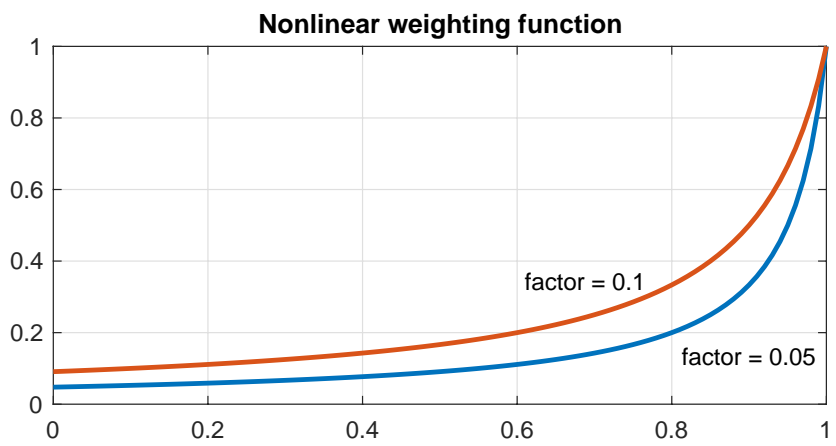

Figure 7: Examples of nonlinear weighting functions 


\section{Placement heuristic hard constraints}

We give details on all hard constraints from Section 3.3.1 of the paper.

\subsection{Hard constraints used in Stage 1}

The following hard constraints must be satisfied in Stage 1, and in some cases other stages, as outlined in Table 2 in the paper.

Constraint 1 (Stages: 1, 2, 3, 4)

The hard constraint for placing a box entirely inside the container.

Constraint 1 is

$$
\begin{cases}\text { false } & \text { if outer-right-upper corner of box exceeds container } \\ & \text { length, width or height, respectively } \\ \text { true } & \text { otherwise. }\end{cases}
$$

Constraint 2 (Stages: 1, 2, 3, 4)

The hard constraint for placing a box such that it does not intersect with other boxes. Constraint 2 is

$$
\begin{cases}\text { true } & \text { if all per box checks return true } \\ \text { false otherwise. }\end{cases}
$$

Check per box is

$$
\begin{cases}\text { true } & \text { if inner-left-lower corner of current box exceeds, in } \\ & \text { any direction, outer-right-upper corner of other box, } \\ \text { or } & \text { if inner-left-lower corner of other box exceeds, in any } \\ & \text { direction, outer-right-upper corner of current box, } \\ \text { false } & \text { otherwise. }\end{cases}
$$


Constraint 3 (Stages: 1, 2, 3, 4)

The hard constraint that a box can have only two orientations (to comply with "this way up"). Constraint 3 is

$$
\begin{cases}\text { true } & \text { if box height is orthogonal to container floor, } \\ \text { false otherwise. }\end{cases}
$$

This constraint is considered when potential placements are generated. Hence, all potential placements will satisfy this constraint.

\section{Constraint 4 (Stages: 1, 2, 3, 4)}

The hard constraint for placing a box such that stacking strength of boxes below is not exceeded. Specifically, factors of importance are the supporting areas of the boxes below, the position of the placed box relative to the edges of the supporting boxes, and the bearing capacities of the supporting boxes. The evaluation of this constraint requires an analysis of the stacking strength of each of the boxes that are supporting from below. Constraint 4 is

$$
\begin{cases}\text { true } & \text { if box is on floor, } \\ \text { or } & \text { if box is above floor and all per area checks are true, } \\ \text { false } & \text { otherwise. }\end{cases}
$$

Check per area is

$$
\begin{cases}\text { false } & \text { if sum of weight on area }> \\ & \max \{\text { best-edge-max-load, lid-max-load }\}, \\ \text { true } & \text { otherwise. }\end{cases}
$$

Here, best-edge-max-load is based on the MaxLoad parameter obtained from the McKee formula (2) in the paper, while lid-max-load refers to the box attribute "strength of packaging material if it provides support with only lid" (which is attribute 3 in Section 2.1 of the paper). Both are converted from lbs to kg. Typically, best-edge-max-load > lid-max-load holds if there is support from edges.

It is necessary to distinguish which edges that provide support: parallel to the container length $(\mathrm{L})$ or container width $(\mathrm{W})$. Figure 8 illustrates all support cases, where a darker box is stacked on top of a lighter box. When needed, an asterisk is used to distinguish left from right and behind from in 


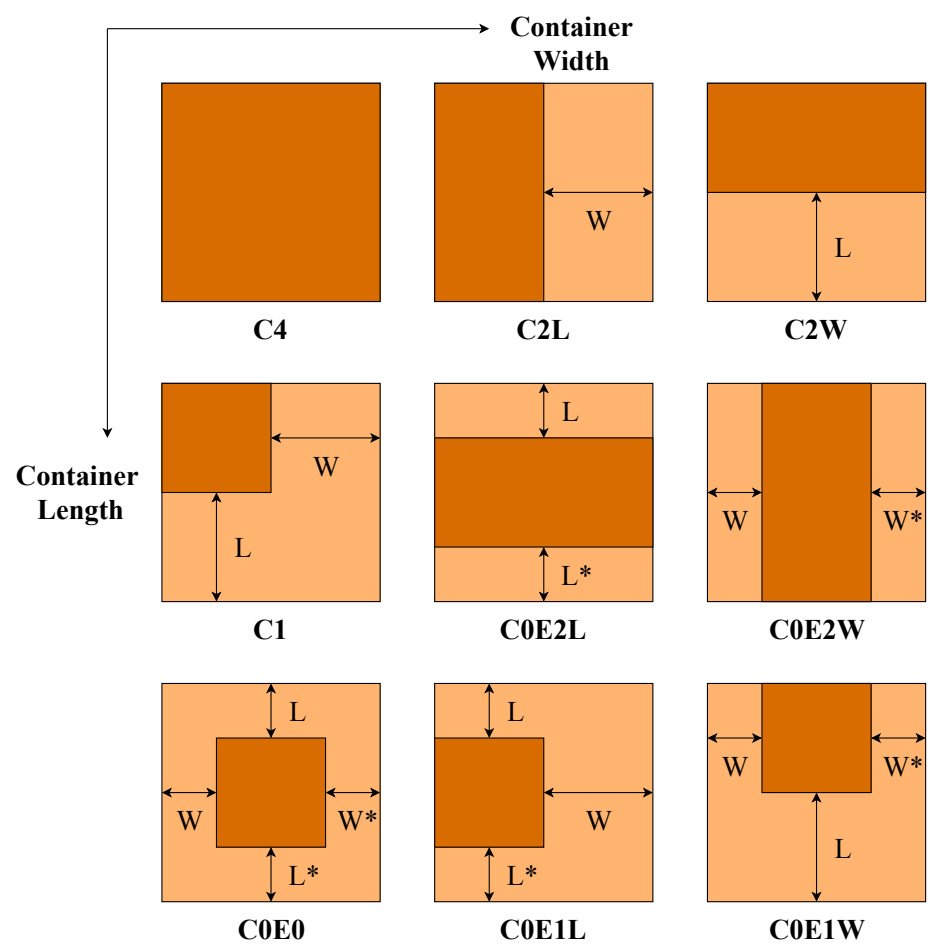

Figure 8: Support cases for the stacking strength constraint. The darker box is stacked on top of the lighter box. (1.5 columns)

front. The labels introduced in the figure are used henceforth. Recall that a logistic function of the distance between the end of the box above and the edge of the box below is used to model partial degree of support; the best-edge-max-load quantity is a consequence of this.

In order to describe how to calculate MaxLoad, here abbreviated ML, each of the cases in Figure 8 must be considered separately. Recalling the McKee formula, we then have

$$
\mathrm{ML}(\text { case })=\frac{5.87 \times E C T \times \sqrt{\text { Thickness } \times \text { Perimeter }(\text { case })}}{\text { EnvirFactor }(\text { case })},
$$

for cases C4, C2L, C2W, C1, COE2L, and COE2W.

- The calculation of Perimeter $(\cdot)$ depends on these six cases; for example, Perimeter $(\mathrm{C} 4)=2 \times$ Area Length $+2 \times$ Area Width, while Perimeter $(\mathrm{C} 1)=1 \times$ Area Length $+1 \times$ Area Width, converted from meter to inches. 
- The environmental factor is calculated as

$$
\begin{aligned}
& \text { EnvirFactor }(\text { case })= \\
& \quad 3.5 \times \text { Envir Factor }_{\text {edges }}(\text { case }) \times \text { Envir Factor }_{\text {overlap }},
\end{aligned}
$$

where EnvirFactor edges (case) is given by

$$
\left\{\begin{array}{l}
1.0 \text { if support from } 4 \text { corners }(\mathrm{C} 4), \\
1.1 \text { if support from } 2 \text { corners }(\mathrm{C} 2 \mathrm{~L} / \mathrm{W}) \text { and } \\
\text { the box is crate of wood, } \\
1.2 \text { if support from } 2 \text { corners (C2L/W) and } \\
\text { the box is of corrugated board, } \\
1.1 \text { if support from } 0 \text { corners but } 2 \text { edges } \\
(\mathrm{COE} 2 \mathrm{~L} / \mathrm{W}) \text { and the box is crate of wood, } \\
1.2 \text { if support from } 0 \text { corners but } 2 \text { edges } \\
(\mathrm{COE} 2 \mathrm{~L} / \mathrm{W}) \text { and the box is of corrugated } \\
\text { board, } \\
2.2 \text { if support from } 1 \text { corner }(\mathrm{C} 1) \text { and the } \\
\text { box is crate of wood, } \\
\text { if support from } 1 \text { corner }(\mathrm{C} 1) \text { and the } \\
\text { box is of corrugated board, }
\end{array}\right.
$$

and EnvirFactor overlap $_{\text {is }}$ given by

$$
\begin{cases}0.33 \quad \text { if support is given from more than one } & \text { box both lengthwise and widthwise, } \\ 0.67 \quad \text { if support is given from more than one } \\ \text { box either lengthwise or widthwise, } \\ 1.00 \quad \text { otherwise. }\end{cases}
$$

Note that the last factor depends on the number of supporting boxes.

The best-edge-max-load quantity in (6b) for all cases in Figure 8 is given in Table 1. Here, the abbreviation $\operatorname{LF}(\cdot)$ means Logistic $(\cdot, 0.875,50)$. Further, parameters $D E_{M}$. are specified per case in Table 2. For the three cases COE0, COE1L, and COE1W, which are not defined in $(6 \mathrm{~d})$, the best-edge-max-load quantities in (6b) will be based on values of $\operatorname{ML}(\cdot)$ for the cases in (6d). In Table 2, the effect of the Logistic function is to cancel out the contribution 
Table 1: Specification of the best-edge-max-load quantity for each of the nine cases. The distance between the end of the box above and the edge of the box below is normalized by dividing by the full length or width of the box below, and they are denoted $D E_{L}$ and $D E_{W}$, respectively.

\begin{tabular}{|c|c|}
\hline Case & best-edge-max-load $=$ maximum of \\
\hline C4 & $\mathrm{ML}(\mathrm{C} 4)$ \\
\hline $\mathrm{C} 2 \mathrm{~L}$ & $\mathrm{ML}(\mathrm{C} 2 \mathrm{~L})+(\mathrm{ML}(\mathrm{C} 4)-\mathrm{ML}(\mathrm{C} 2 \mathrm{~L})) \times \mathrm{LF}\left(1-D E_{W}\right)$ \\
\hline $\mathrm{C} 2 \mathrm{~W}$ & $\mathrm{ML}(\mathrm{C} 2 \mathrm{~W})+\left(\mathrm{ML}(\mathrm{C} 4)-\mathrm{ML}(\mathrm{C} 2 \mathrm{~W}) \times \operatorname{LF}\left(1-D E_{L}\right)\right.$ \\
\hline $\mathrm{C} 1$ & $\begin{array}{l}\mathrm{ML}(\mathrm{C} 1)+(\mathrm{ML}(\mathrm{C} 2 \mathrm{~L})-\mathrm{ML}(\mathrm{C} 1)) \times \mathrm{LF}\left(1-D E_{L}\right) \\
\mathrm{ML}(\mathrm{C} 1)+(\mathrm{ML}(\mathrm{C} 2 \mathrm{~W})-\mathrm{ML}(\mathrm{C} 1)) \times \mathrm{LF}\left(1-D E_{W}\right) \\
\mathrm{ML}(\mathrm{C} 1)+(\mathrm{ML}(\mathrm{C} 4)-\mathrm{ML}(\mathrm{C} 1)) \times \mathrm{LF}\left(1-D E_{M}\right)\end{array}$ \\
\hline COE2L & $\begin{array}{l}\mathrm{ML}(\mathrm{COE} 2 \mathrm{~L})+(\mathrm{ML}(\mathrm{C} 2 \mathrm{~W})-\mathrm{ML}(\mathrm{COE} 2 \mathrm{~L})) \times \mathrm{LF}\left(1-D E_{L}\right) \\
\mathrm{ML}(\mathrm{COE} 2 \mathrm{~L})+(\mathrm{ML}(\mathrm{C} 2 \mathrm{~W})-\mathrm{ML}(\mathrm{COE} 2 \mathrm{~L})) \times \mathrm{LF}\left(1-D E_{L *}\right) \\
\mathrm{ML}(\mathrm{COE} 2 \mathrm{~L})+(\mathrm{ML}(\mathrm{C} 4)-\mathrm{ML}(\mathrm{COE} 2 \mathrm{~L})) \times \mathrm{LF}\left(1-D E_{M}\right)\end{array}$ \\
\hline $\mathrm{COE} 2 \mathrm{~W}$ & $\begin{array}{l}\mathrm{ML}(\mathrm{COE} 2 \mathrm{~W})+(\mathrm{ML}(\mathrm{C} 2 \mathrm{~L})-\mathrm{ML}(\mathrm{COE} 2 \mathrm{~W})) \times \mathrm{LF}\left(1-D E_{W}\right) \\
\mathrm{ML}(\mathrm{COE} 2 \mathrm{~W})+(\mathrm{ML}(\mathrm{C} 2 \mathrm{~L})-\mathrm{ML}(\mathrm{COE} 2 \mathrm{~W})) \times \mathrm{LF}\left(1-D E_{W *}\right) \\
\mathrm{ML}(\mathrm{COE} 2 \mathrm{~W})+(\mathrm{ML}(\mathrm{C} 4)-\mathrm{ML}(\mathrm{COE} 2 \mathrm{~W})) \times \mathrm{LF}\left(1-D E_{M}\right)\end{array}$ \\
\hline COEO & $\begin{array}{l}\mathrm{ML}(\mathrm{COE} 2 \mathrm{~L}) \times \operatorname{LF}\left(1-D E_{M a}\right) \\
\mathrm{ML}(\mathrm{COE} 2 \mathrm{~W}) \times \operatorname{LF}\left(1-D E_{M b}\right) \\
\operatorname{ML}(\mathrm{C} 1) \times \operatorname{LF}\left(1-D E_{M c}\right) \\
\operatorname{ML}(\mathrm{C} 2 \mathrm{~L}) \times \operatorname{LF}\left(1-D E_{M d}\right) \\
\operatorname{ML}(\mathrm{C} 2 \mathrm{~W}) \times \operatorname{LF}\left(1-D E_{M e}\right) \\
\operatorname{ML}(\mathrm{C} 4) \times \operatorname{LF}\left(1-D E_{M f}\right)\end{array}$ \\
\hline COE1L & $\begin{array}{l}\mathrm{ML}(\mathrm{COE} 2 \mathrm{~L}) \times \operatorname{LF}\left(1-D E_{W}\right) \\
\operatorname{ML}(\mathrm{C} 1) \times \operatorname{LF}\left(1-D E_{M a}\right) \\
\operatorname{ML}(\mathrm{C} 2 \mathrm{~L}) \times \operatorname{LF}\left(1-D E_{M b}\right) \\
\operatorname{ML}(\mathrm{C} 2 \mathrm{~W}) \times \operatorname{LF}\left(1-D E_{M c}\right) \\
\operatorname{ML}(\mathrm{C} 4) \times \operatorname{LF}\left(1-D E_{M d}\right)\end{array}$ \\
\hline $\mathrm{COE} 1 \mathrm{~W}$ & $\begin{array}{l}\operatorname{ML}(\mathrm{COE} 2 \mathrm{~W}) \times \operatorname{LF}\left(1-D E_{L}\right) \\
\operatorname{ML}(\mathrm{C} 1) \times \operatorname{LF}\left(1-D E_{M a}\right) \\
\operatorname{ML}(\mathrm{C} 2 \mathrm{~W}) \times \operatorname{LF}\left(1-D E_{M b}\right) \\
\operatorname{ML}(\mathrm{C} 2 \mathrm{~L}) \times \operatorname{LF}\left(1-D E_{M c}\right) \\
\operatorname{ML}(\mathrm{C} 4) \times \operatorname{LF}\left(1-D E_{M d}\right)\end{array}$ \\
\hline
\end{tabular}

from partial degree of support if the distance between the end of the box above and the edge of the box below is too long. 
Table 2: Specification of the auxiliary parameters $D E_{M(\cdot)}$ for each of the six cases C1, COE2L, COE2W, COEO, COE1L, and COE1W. These parameters are used when calculating the best-edge-max-load quantities in Table 1 .

\begin{tabular}{l|l}
\hline Case & Calculation \\
\hline \hline C1 & $D E_{M}=\max \left\{D E_{L}, D E_{W}\right\}$ \\
\hline COE2L & $D E_{M}=\max \left\{D E_{L}, D E_{L *}\right\}$ \\
\hline COE2W & $D E_{M}=\max \left\{D E_{W}, D E_{W *}\right\}$ \\
\hline COE0 & $D E_{M a}=\max \left\{D E_{W}, D E_{W *}\right\}$ \\
& $D E_{M b}=\max \left\{D E_{L}, D E_{L *}\right\}$ \\
& $D E_{M c}=\max \left\{\min \left\{D E_{W}, D E_{W *}\right\}, \min \left\{D E_{L}, D E_{L *}\right\}\right\}$ \\
& $D E_{M d}=\max \left\{D E_{M b}, \min \left\{D E_{W}, D E_{W *}\right\}\right\}$ \\
& $D E_{M e}=\max \left\{D E_{M a}, \min \left\{D E_{L}, D E_{L *}\right\}\right\}$ \\
& $D E_{M f}=\max \left\{D E_{M a}, D E_{M b}\right\}$ \\
\hline COE1L & $D E_{M a}=\min \left\{D E_{L}, D E_{L *}\right\}$ \\
& $D E_{M b}=\max \left\{D E_{L}, D E_{L *}\right\}$ \\
& $D E_{M c}=\max \left\{D E_{M a}, D E_{W}\right\}$ \\
& $D E_{M d}=\max \left\{D E_{M b}, D E_{W}\right\}$ \\
\hline COE1W & $D E_{M a}=\min \left\{D E_{W}, D E_{W *}\right\}$ \\
& $D E_{M b}=\max \left\{D E_{W}, D E_{W *}\right\}$ \\
& $D E_{M c}=\max \left\{D E_{M a}, D E_{L}\right\}$ \\
& $D E_{M d}=\max \left\{D E_{M b}, D E_{L}\right\}$ \\
\hline
\end{tabular}

For example, assume that $B 1$ in Figure 8 in the paper is placed in the lower inner left corner of the container. The support from $B 2$ to $B 3$ is then referred to as C2L. To check if any partial support is gained from $B 2$ to $B 3$, the normalized distance in widthwise direction to the rightmost edge of $B 2$ is assessed, denoted $D E_{W}$. As indicated by the red edges of $B 2$, the distance from the end of $B 3$ to the rightmost edge of $B 2$ is too long to gain any partial support. Since the distance is too long, $\operatorname{LF}\left(1-D E_{W}\right)=0$ and hence only $\mathrm{ML}(\mathrm{C} 2 \mathrm{~L})$ is used. Conversely, the support from $B 1$ to $B 2$, classified as $\mathrm{C} 1$ may have $\mathrm{LF}\left(1-D E_{W}\right)>0$ and hence receive contribution of partial support in terms of $\mathrm{C} 2 \mathrm{~W}$, in addition to $\mathrm{ML}(\mathrm{C} 1)$. This is indicated by the blue edges of $B 1$ in Figure 8 in the paper. 
To summarize, to evaluate $6 \mathrm{~b}$ for one area the following steps are used:

- identify the current case according to Figure 8 ,

- compute the quantities $D E_{L}$. and $D E_{W}$. for the current case,

- use the McKee formula and Tables 1 and 2 to calculate the best-edge-max-load quantity for the current case,

- evaluate $(6 \mathrm{~b})$ for the area.

Constraint 5 (Stages: 1, 2, 3, 4)

The hard constraint for not placing a box on top of a different box with the exception "not feasible to stack a different package above". Constraint 5 is

$\begin{cases}\text { true if all per box checks return true, } \\ \text { false otherwise. }\end{cases}$

Check per box is

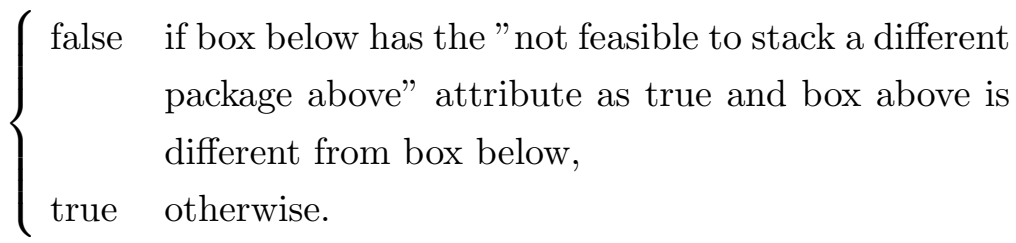

Here, two boxes are assumed to be different if the item(s) contained in them are not exactly the same, or if the item(s) in the boxes are unknown.

In the problem data provided in the Supplementary material data file Input50.csv, it is not specified what items are in each box. This lack of information means that if a box has this exception, nothing can ever be stacked on top of it. This was also the case for the runs reported in Section 4 of the paper, whereas when the load plans were initially created at the DC the item(s) in each box were available in the software. Hence, the problem was slightly less constrained when the software was used at the DC versus when we used it for the computational experiments. 
Constraint 6 (Stages: 1, 2, 3, 4)

The hard constraint for not placing a box on top of a similar box with the exception "not feasible to stack a similar package above". Constraint 6 is

$$
\begin{cases}\text { true } & \text { if all per box checks return true } \\ \text { false } & \text { otherwise. }\end{cases}
$$

Check per box is

$$
\left\{\begin{aligned}
\text { false } & \text { if box below has the "not feasible to stack a similar } \\
& \text { package above" attribute as true and box above is } \\
& \text { similar to box below, } \\
\text { true } & \text { otherwise. }
\end{aligned}\right.
$$

Here, two boxes are assumed to be similar if the item(s) contained in them are exactly the same, or if the item(s) in the boxes are unknown. In the problem data the contents of the boxes are not given, which means that if a box has this exception nothing can ever be stacked on top of it. (Compare with the comment below (7a) $-7 \mathrm{~b}$ ) about availability of item data.)

Constraint 7 (Stages: 1, 4)

The hard constraint for not placing a box with the exception "must be placed on the floor" above the floor. Constraint 7 is

$$
\begin{cases}\text { false } & \text { if box has the "must be placed on the floor" } \\ & \text { attribute as true and box is above floor, } \\ \text { true } & \text { otherwise. }\end{cases}
$$

This constraint is not needed for Stage 2 since this exception is never applicable to consolidation pallets, and not for Stage 3 since boxes to be placed in consolidation pallets never need to be placed on the container floor, see Section 3.2 in the paper. 
Constraint 8 (Stages: 1, 2, 4)

The hard constraint for not placing a box in front of or above a hazardous box. Constraint 8 is

$$
\begin{cases}\text { true } & \text { if all per box checks return true } \\ \text { false otherwise. }\end{cases}
$$

Check per box is

$$
\begin{cases}\text { false } & \begin{array}{l}
\text { if current box is hazardous and other box in front } \\
\text { of it }
\end{array} \\
\text { or } & \text { if hazardous box below or behind current box, } \\
\text { true } & \text { otherwise. }\end{cases}
$$

Whether a box is hazardous is specified by attribute 5 in Section 2.1 of the paper. This constraint is not needed for Stage 3, since boxes to be placed in consolidation pallets never contain hazardous materials, see Section 3.2 in the paper.

Constraint 9 (Stages: 1, 2, 3, 4)

The hard constraint for placing a box with sufficient base support.

Constraint 9 is

$$
\begin{cases}\text { true } & \text { if box on floor, } \\
\text { or } & \begin{array}{l}
\text { if all four corners of box have support below or if two } \\
\text { corners and } 80 \% \text { of base area have support below, }
\end{array} \\
\text { or } \quad \begin{array}{l}
\text { if i) } 80 \% \text { of base area has support below and ii) box } \\
\text { is long and iii) largest dimension is greater than }
\end{array} \\
\begin{array}{l}
1.5 \mathrm{~m} \text { and iv) box weight is less than } 36 \mathrm{~kg}, \\
\text { false }
\end{array}\end{cases}
$$

Here, a box is defined as long if the ratio between its largest and second largest dimension is greater than 2.5. The different criteria in Constraint 9 have evolved based on feedback from DC operators; their purpose is to achieve sufficient vertical stability while not restricting more than necessary. 


\subsection{Hard constraints used only after Stage 1}

The following hard constraints are not used in Stage 1, but only in later stages, as outlined in Table 2 in the paper.

Constraint 10 (Stages: 2)

The hard constraint for the possible height of a consolidation pallet to be greater than a minimum. Constraint 10 is

$$
\left\{\begin{aligned}
\text { false } & \text { if ceiling height }- \text { height from floor } \\
& <\text { consolidation pallet min height } \\
\text { true } & \text { otherwise. }
\end{aligned}\right.
$$

Here, "ceiling height" is the height from the floor to the ceiling, and "height from floor" refers to the height to the bottom surface for the consolidation pallet. Further, "consolidation pallet min height" refers to the lower limit of the height, which is $0.36 \mathrm{~m}$. The purpose is to ensure that at least one layer of the smallest boxes can fit during Stage 3. Note that (12) does not consider if another box is limiting the height. This is taken care of by (4a)(4b), since the height of the consolidation pallet during Stage 2 is set to "consolidation pallet min height". While $4 \mathrm{a}-4 \mathrm{~b}$ ) would be sufficient to ensure that (12) is true, the benefit of also having the latter is to reject an infeasible placement quicker, without the per box checks.

Constraint 11 (Stages: 3)

The hard constraint to ensure that the container (consolidation pallet) weight capacity is not exceeded. Constraint 11 is

$$
\left\{\begin{aligned}
\text { false } & \text { if loaded weight }+ \text { weight current box } \\
& >\text { consolidation pallet max load, } \\
\text { true } & \text { otherwise. }
\end{aligned}\right.
$$

Here, "consolidation pallet max load" is the maximum possible weight of the consolidation pallet based on where it will be placed. This limit is set during Stage 2, to ensure that the stacking strength constraints 6a -6e will be satisfied in Stage 4 (see Figure 6 in the paper). 


\section{Placement heuristic scoring components}

We give details on all scoring components from Section 3.3.2 of the paper.

\subsection{Scoring used in Stage 1}

The following scoring components are used in Stage 1, and in some cases other stages, as outlined in Table 3 in the paper.

Scoring 1 (Stages: 1, 3, 4)

The scoring component for placing a heavy box close to the floor is

$$
s_{1}(k)=\left(1-\frac{\text { height from floor }}{\text { container height }}\right) \times \frac{\text { weight of box }}{\text { max weight }} .
$$

Here, "height from floor" is the vertical position of the center of gravity for the box if placed at the container point, and "max weight" is the weight of the heaviest box in the current stage. (It is always assumed that the weight of a box is evenly distributed.)

Scoring 2 (Stages: 1, 2, 3, 4)

The scoring component for placing a box close to the floor, regardless of weight, is

$$
s_{2}(k)=1-\frac{\text { height from floor }}{\text { container height }} .
$$

Here, "height from floor" is the vertical position of the bottom surface for the box if placed at the container point.

Scoring 3 (Stages: 1, 4)

The scoring component for placing a box close to the intersection of the inner wall and the ceiling lengthwise is

$$
s_{3}(k)=\left(1-\frac{\text { length from inner wall }}{\text { container length }}\right) \times \frac{\text { height from floor }}{\text { container height }} .
$$

Here, "length from inner wall" is the horizontal position lengthwise of the side surface (the side which is furthest away from the inner wall of the container) for the box if placed at the container point. Similarly, "height from floor" is the vertical position of the top surface for the box. 
Scoring 4 (Stages: 1, 3, 4)

The scoring component for placing a box with high stacking strength, when providing support with edges/corners, close to the floor is

$$
s_{4}(k)=\min \left\{1, \frac{E C T \times \sqrt{\text { Thickness }}}{50}\right\} \times\left(1-\frac{\text { height from floor }}{\text { container height }}\right) .
$$

Here, ECT and Thickness are from the McKee formula (2) in the paper, where $E C T$ inherited from the packaging has been adjusted based on the exception, if applicable, of reduced or increased stacking strength (attribute 10 in Section 2.1 of the paper). The value 50 in the denominator was chosen based on typical values of the numerator. The "height from floor" is the vertical position of the top surface for the box.

Scoring 5 (Stages: 1, 3, 4)

The scoring component for placing a box with high stacking strength, when providing support with only lid, close to the floor is

$$
s_{5}(k)=\min \left\{1, \frac{\text { strength of lid }}{150}\right\} \times\left(1-\frac{\text { height from floor }}{\text { container height }}\right) .
$$

Here, "strength of lid" refers to attribute 3 in Section 2.1 of the paper, that is, strength of packaging material if the box provides support to a box above with only its lid. It is typically in the range $[10,150]$ lbs (based on experiments) and, similar to the adjustment of $E C T$, it is adjusted according to the exception of reduced or increased stacking strength, if applicable. The "height from floor" is the vertical position of the top surface for the box.

Scoring 6 (Stages: 1, 2, 3, 4)

The scoring component for space utilization lengthwise, prioritizing leaving little space unused, is

$$
s_{6}(k)=\text { Weighting }\left(\min \left\{1, \frac{\text { box length }}{\text { support length }}\right\}, 0.05\right) .
$$

Here, "support length" is the length of an area, on the floor or on top of other boxes, which can give support; see the example in Section 3.3.2 of the paper. The purpose of the nonlinear weighting function is to reward high space utilization even more, and barely reward low space utilization at all. 
Scoring 7 (Stages: 1, 2, 3, 4)

The scoring component for space utilization widthwise, prioritizing leaving little space unused, is

$$
s_{7}(k)=\text { Weighting }\left(\min \left\{1, \frac{\text { box width }}{\text { support width }}\right\}, 0.05\right) .
$$

Here, "support width" is the width of an area, on the floor or on top of other boxes, which can give support. This component is very similar to 19 .

Scoring 8 (Stages: 1, 2, 3, 4)

The scoring component for space utilization heightwise, prioritizing leaving little space unused, is

$$
s_{8}(k)=\text { Weighting }\left(\min \left\{1, \frac{\text { box height }}{\text { support height }}\right\}, 0.05\right) .
$$

Here, "support height" is the height from the supporting area, that is, the floor or the top of other boxes, to the container ceiling or a box above (due to overhang). This component is also very similar to (19).

Scoring 9 (Stages: 1, 2, 3, 4)

The scoring component for placing a box with support from behind is

$$
s_{9}(k)=\operatorname{Logistic}\left(\frac{\text { support- } \mathrm{from}-\text { behind }}{\text { area of box back side }}, 0.3,20\right) .
$$

Here, support-from-behind is the contact area (with other boxes or the container walls behind the box) and the logistic function is used to capture a threshold effect for what makes up sufficient support. 
Scoring 10 (Stages: 1, 2, 3, 4)

The scoring component for placing a box with support from in front, left and right, is

$$
\begin{aligned}
s_{10}(k)=\frac{1}{3} \times[ & \operatorname{Logistic}\left(\frac{\text { support-from-front }}{\text { area of box front side }}, 0.3,20\right)+ \\
& \text { Logistic }\left(\frac{\text { support-from-left }}{\text { area of box left side }}, 0.3,20\right)+ \\
& \text { Logistic } \left.\left(\frac{\text { support-from-right }}{\text { area of box right side }}, 0.3,20\right)\right] .
\end{aligned}
$$

Here, support-from-front, support-from-left, support-from-right are similar to support-from-behind in (22).

Scoring 11 (Stages: 1, 2, 3, 4)

The scoring component for placing a box at a backward adjusted duplicate point is

$$
s_{11}(k)= \begin{cases}1 & \text { if backward adjusted lengthwise or widthwise } \\ 0.25, & \text { if backward adjusted diagonally } \\ 0 & \text { otherwise }\end{cases}
$$

Here, "backward adjusted" refers to a container point that was created by duplicating and adjusting a normal or a projected point backward in order to avoid intersection with the container walls or other boxes, as outlined in Section 3.3 of the paper. The lower value for diagonally backward adjusted points reflects that such adjustment can be more disruptive, for example by hindering later placements. 
Scoring 12 (Stages: 1, 2, 3, 4)

The scoring component for placing a box at a forward adjusted duplicate point is

$$
s_{12}(k)= \begin{cases}1 & \text { if forward adjusted lengthwise or widthwise } \\ 0.25, & \text { if forward adjusted diagonally } \\ 0 & \text { otherwise }\end{cases}
$$

Here, "forward adjusted" refers to a container point that was created by duplicating and adjusting a normal or a projected point forward in order to gain stacking strength, as outlined in Section 3.3 of the paper. Similar to (24), the lower value for diagonally forward adjusted points reflects that such adjustment can be more disruptive.

Scoring 13 (Stages: 1, 3, 4)

The scoring component for placing a box ending at same height as an adjacent box to the left is

$$
s_{13}(k)=\frac{\text { support-from-left-at-same-height }}{\text { box length }} .
$$

Here, support-from-left-at-same-height is the length of the contact area (with other boxes) that ends at the same height as the top surface for the box being placed. Putting boxes of the same height together enables stacking with overlap.

Scoring 14 (Stages: 1, 3, 4)

The scoring component for placing a box with the same height as many other boxes is

$$
s_{14}(k)=\frac{\text { boxes with same height }}{10} .
$$

Here, "boxes with same height" is the number of boxes in the current stage that have the same height as the box being placed. This component can, for example, contribute to that boxes of similar height are being placed close to each other. The denominator 10 was chosen based on characteristics of the studied shipments (see problem data file Input50.csv); only seven out of the 50 instances have more than 10 packages with the same height in Stage 1, and the highest number of packages with the same height in Stage 1 is 22 . 
Scoring 15 (Stages: 1, 4)

The scoring component for placing a box on top of a similar box with the exception "not feasible to stack a different package above" is

$$
s_{15}(k)= \begin{cases}1 & \begin{array}{l}
\text { if box below has the "not feasible to stack a } \\
\text { different package above" attribute as true } \\
0 \quad \text { otherwise }
\end{array}\end{cases}
$$

Here, two boxes are assumed to be similar if the item(s) contained in them are exactly the same. For the purpose of the scoring, it is sufficient to check the stacking attribute of the box below, since if the boxes were different the placement would already have been rejected based on the related hard constraint in Table 2 in the paper.

Scoring 16 (Stages: 1)

The scoring component for placing a box with the exception "must be placed on the floor" is

$$
s_{15}(k)= \begin{cases}0.2 \quad \begin{array}{l}
\text { if box has the "must be placed on the floor" } \\
\text { attribute as true } \\
0 \quad \text { otherwise }
\end{array}\end{cases}
$$

Here, for the purpose of the scoring, it is sufficient to check the attribute, since if the point was not on the floor the placement would already have been rejected based on the related hard constraint in Table 2 in the paper. 
Scoring 17 (Stages: 1)

The scoring component for placing a box considered to be long, with the largest dimension greater than $1.5 \mathrm{~m}$, is

$$
s_{17}(k)=\left\{\begin{array}{l}
1 \quad \begin{array}{l}
\text { if box long and the largest dimension greater } \\
\text { than } 1.5 \mathrm{~m}, \\
0 \quad \text { otherwise }
\end{array}
\end{array}\right.
$$

Here, a box is considered to be long if the ratio between its largest and second largest dimension is greater than 2.5. Boxes that meet the two criteria in (30) can be difficult to stack on top of other boxes, since this may require overlap of two or more boxes below. At the same time, boxes that meet these two criteria take up valuable floor space if placed directly on the floor. This component and the value of the corresponding scoring weight will guide the placement heuristic to load long boxes early or later on.

\section{Scoring 18 (Stages: 1, 4)}

The scoring component for placing a hazardous box closer to the intersection of the door and the ceiling lengthwise, or a box with the exceptions "not feasible to stack a different package above" or "not feasible to stack a similar package above" closer to the ceiling, is

$s_{18}(k)= \begin{cases}1-\text { pos } & \text { if box hazardous, } \\ \frac{\text { height from floor }}{\text { container height }} & \text { if different-or-similar-box-attribute } \\ 0 & \text { otherwise. }\end{cases}$

where

$$
\text { pos }:=\frac{\sqrt{\begin{array}{c}
(\text { container length }- \text { length from inner wall })^{2} \\
+(\text { container height }- \text { height from floor })^{2}
\end{array}}}{\sqrt{(\text { container length })^{2}+(\text { container height })^{2}}} .
$$

Here, the length and height terms are the same as in (16), while differentor-similar-box-attribute refers to if the box has the "not feasible to stack a different package above" attribute, or the "not feasible to stack a similar package above" attribute, as true. 
Scoring 19 (Stages: 1, 2, 4)

The scoring component for placing a box with pallet not rotated (relative to the direction of the pallet runners) is

$$
s_{19}(k)= \begin{cases}0, & \text { if box has pallet and box orientation not same } \\ & \text { as direction of pallet runners } \\ 0.5, & \text { otherwise }\end{cases}
$$

Here, the purpose is to penalize a placement where the operator would have to push and rotate the package using the forklift or by hand.

Scoring 20 (Stages: 1, 4)

The scoring component for placing a box without pallet not on the floor is

$$
s_{20}(k)= \begin{cases}0, & \text { if box without pallet on floor }, \\ 0.5, & \text { otherwise }\end{cases}
$$

Here, the purpose is to penalize a placement where the operator potentially would have to place some additional material below the package in order to protect it from moisture.

\subsection{Scoring used only after Stage 1}

The following scoring components are not used in Stage 1, but only in later stages, as specified in Table 3 in the paper.

Scoring 21 (Stages: 2)

The scoring component for maximum possible height of a consolidation pallet based on where it will be placed is

$$
s_{21}(k)=\min \left\{1, \frac{\text { box-above-or-ceiling-height }- \text { (height from floor })}{\text { consolidation pallet max height }}\right\}
$$

Here, box-above-or-ceiling-height is the height from the floor to the bottom surface for a package above the consolidation pallet, or to the ceiling if there is no package above. Second, "height from floor" refers to the height to the bottom surface for the consolidation pallet. Third, "consolidation pallet max height" refers to the upper limit of the height (to ensure horizontal stability) and is based on the kind of consolidation pallet being 
considered. The purpose is to promote placements where sufficient volume, limited by the height, can be loaded on the consolidation pallet in Stage 3 while satisfying the basic geometric constraints in Stage 4. The "consolidation pallet max height" is in the interval $[0.965,1.524]$ meters.

Scoring 22 (Stages: 2)

The scoring component for maximum possible weight of a consolidation pallet based on where it will be placed is

$$
s_{22}(k)=\min \left\{1, \frac{\text { consolidation pallet max load }}{200}\right\} .
$$

Here, "consolidation pallet max load" in $\mathrm{kg}$ is based on the McKee formula (2) in the paper, and its purpose is to promote placements where sufficient weight can be loaded on the consolidation pallet in Stage 3 without exceeding the hard stacking strength constraint in Stage 4 (see Figure 6 in the paper). The denominator $200 \mathrm{~kg}$ was deemed appropriate considering Stage 2 packages in the problem instances, and the volume capacity of consolidation pallets.

Scoring 23 (Stages: 4)

The scoring component for placing a consolidation pallet at its predetermined point from Stage 2 is

$$
s_{23}(k)=\left\{\begin{array}{cl}
10, & \text { if consolidation pallet at point from Stage 2, } \\
0, & \text { otherwise }
\end{array}\right.
$$

Here, it is ensured that the filled consolidation pallets are placed at the predetermined points from Stage 2. Note that the scoring function (1) in the paper is designed to give priority to this scoring component. 


\section{Load plan evaluation fitness components}

We give details on all fitness components from Section 3.4 in the paper.

\subsection{Fitness used in Stage 1}

The following fitness components are used in Stage 1, and in some cases other stages, as outlined in Table 4 in the paper.

Fitness 1 (Stages: 1, 2, 3, 4)

The fitness component for no leftover boxes is

$$
f_{1}(i)= \begin{cases}1, & \text { if no leftover boxes } \\ 0, & \text { otherwise }\end{cases}
$$

Fitness 2 (Stages: 1, 2, 3, 4)

The fitness component for minimizing the number of leftover boxes is

$$
f_{2}(i)=\frac{\text { loaded number of boxes }}{\text { total number of boxes }} \text {. }
$$

Fitness 3 (Stages: 1, 2, 3, 4)

The fitness component for minimizing the volume of leftover boxes is

$$
f_{3}(i)=\frac{\text { loaded volume }}{\text { total shipment volume }} .
$$

Fitness 4 (Stages: 1, 2, 3, 4)

The fitness component for minimizing the weight of leftover boxes is

$$
f_{4}(i)=\frac{\text { loaded weight }}{\text { total shipment weight }} .
$$


Fitness 5 (Stages: 1)

The fitness component for promising spaces for consolidation pallets is

$$
f_{5}(i)=\min \left\{1, \frac{\text { consolidation pallet spaces }}{3}\right\} \text {. }
$$

Here, "consolidation pallet spaces" is the number of spaces where the selected consolidation pallet type(s) can fit in terms of base area. These are candidates for placements in Stage 2, when the remaining hard constraints will be checked. The denominator 3 reflects that typically three or less consolidation pallets are needed accommodate the Stage 3 packages.

Fitness 6 (Stages: 1, 2, 3, 4)

The fitness component for maximizing the support from behind for boxes is

$$
f_{6}(i)=\frac{\text { sum of support-from-behind-measure over all boxes }}{\text { loaded number of boxes }} .
$$

Here, the support-from-behind-measure is the value of a logistic function of the contact area (with other boxes or the container walls behind the box) divided by the total area of the box's back side. This is calculated in the same way as the corresponding scoring component $(22)$.

Fitness 7 (Stages: 1, 2, 3, 4)

The fitness component for maximizing the support from in front, left and right for boxes, is

$f_{7}(i)=\frac{\text { sum of support-from-front-left-right-measure over all boxes }}{\text { loaded number of boxes }}$.

Here, the support-from-front-left-right-measure is very similar to the support-from-behind-measure in (42), and it is calculated in the same way as the corresponding scoring component (23). 
Fitness 8 (Stages: 1, 2, 3)

The fitness component for maximizing the utilization of floor space is

$$
f_{8}(i)=\frac{\text { sum of } \text { floor-space-utilization-per-container }}{\text { number of containers }} .
$$

Here, the floor-space-utilization-per-container is the sum of base area over all boxes placed directly on the floor divided by the base area of the container, calculated per container. Recall that for Stage 3 there is often more than one container, that is, consolidation pallet, while in the other stages there is only one shipping container.

Fitness 9 (Stages: 1, 2, 4)

The fitness component for a box not on the floor to have support from behind, if it is non-slim, or support from behind or in front, if it is slim, is

$$
f_{9}(i)=\frac{\text { number of boxes that satisfy the support criterion }}{\text { loaded number of boxes }}
$$

where the support criterion is

$$
\begin{cases}\text { false } & \text { if i) box more than } 0.25 \mathrm{~m} \text { above floor and ii) } \\ & \text { box slim and iii) support from behind, or in } \\ & \text { front, is less than } 10 \% \text { of side area, } \\ \text { or } & \text { if i) box more than } 0.25 \mathrm{~m} \text { above floor and ii) } \\ & \text { box not slim and iii) support from behind is } \\ & \text { less than } 10 \% \text { of side area, } \\ \text { true } & \text { otherwise. }\end{cases}
$$

Here, a box is considered to be slim if the ratio between its height and the smaller of its length and width is greater than 1.75. The purpose of this component is to penalize unstable box configurations, such as a tower of packages, which may require the use of securing materials to prevent the packages from sliding and falling during transport. 
Fitness 10 (Stages: 1)

The fitness component for the center of gravity lengthwise to be closer to the center of the container is

$$
f_{10}(i)=\frac{\text { sum of center-of-gravity-lengthwise-measure }}{\text { number of containers }},
$$

where the center-of-gravity-lengthwise-measure is

$$
\operatorname{Logistic}\left(1-\frac{\mid \text { COG-lengthwise }-(\text { container length }) / 2 \mid}{(\text { container length }) / 2}, 0.7,50\right) \text {, }
$$

with COG-lengthwise :=

$$
\frac{\text { sum of }(\text { box weight } \times \text { length from inner wall })}{\text { sum of box weight }} .
$$

Here, the "length from inner wall" is the horizontal position lengthwise of the center of gravity for the box. (It is always assumed that the weight of a box is evenly distributed.) This component is designed to work for multiple containers, even though in Stage 1 the number of containers is always one. The purpose of the logistic function is to capture a threshold effect for what is close enough to the mid-point of the container. 
Fitness 11 (Stages: 1)

The fitness component for the center of gravity widthwise to be closer to the center of the container is

$$
f_{11}(i)=\frac{\text { sum of center-of-gravity-widthwise-measure }}{\text { number of containers }},
$$

where the center-of-gravity-widthwise-measure is

$$
\operatorname{Logistic}\left(1-\frac{\mid \text { COG-widthwise }-(\text { container width }) / 2 \mid}{(\text { container width }) / 2}, 0.8,50\right) \text {, }
$$

with COG-widthwise :=

$$
\frac{\text { sum of (box weight } \times \text { length from left wall) }}{\text { sum of box weight }} .
$$

Here, the "length from left wall" is the horizontal position widthwise of the center of gravity for the box. (It is always assumed that the weight of a box is evenly distributed.) This component is very similar to (46a)-(46c). Note that the parameters of the logistic function differ.

Fitness 12 (Stages: 1)

The fitness component for the center of gravity heightwise to be closer to the floor of the container is

$$
f_{12}(i)=\frac{\text { sum of center-of-gravity-heightwise-measure }}{\text { number of containers }},
$$

where center-of-gravity-heightwise-measure is

$$
\text { Logistic }\left(1-\frac{\text { COG-heightwise }}{\text { container height }}, 0.5,20\right) \text {, }
$$

with COG-heightwise :=

$$
\frac{\text { sum of (box weight } \times \text { height from floor })}{\text { sum of box weight }} .
$$

Here, the "height from floor" is the vertical position of the center of gravity for the box. (It is always assumed that the weight of a box is evenly distributed.) This component is also very similar to (46a)-(46c). Note that the parameters of the logistic function differ. 
Fitness 13 (Stages: 1, 2, 4)

The fitness component for boxes with pallet not rotated is

$$
f_{13}(i)=\frac{\text { number of boxes not pallet rotated }}{\text { loaded number of boxes }} .
$$

Here, "pallet rotated" is checked in the same way as in the corresponding scoring component 32 .

Fitness 14 (Stages: 1, 2, 4)

The fitness component for boxes with pallet reachable by forklift is

$$
f_{14}(i)=\frac{\text { number of boxes reachable by forklift }}{\text { loaded number of boxes }},
$$

where "reachable by forklift" is

$$
\left\{\begin{aligned}
\text { false } & \text { if box has pallet and the forks can access less } \\
& \text { than } 20 \% \text { of its length, } \\
\text { true } & \text { otherwise. }
\end{aligned}\right.
$$

Here, the access of the forks is limited by other packages, in front of the current package, that are blocking the truck.

Fitness 15 (Stages: 1, 4)

The fitness component for boxes without pallet not on the floor is

$$
f_{15}(i)=\text { Weighting }\left(\frac{\text { number of boxes not without pallet on floor }}{\text { loaded number of boxes }}, 0.02\right)
$$

Here, "without pallet on floor" is checked in the same way as in the corresponding scoring component (33). The purpose of using the nonlinear weighting function is to further penalize this kind of awkward placement. 


\subsection{Fitness used only after Stage 1}

The following fitness components are not used in Stage 1, but only in Stage 2, as outlined in Table 4 in the paper.

Fitness 16 (Stages: 2)

The fitness component for maximum possible height of consolidation pallets based on where they will be placed is

$$
f_{16}(i)=\frac{\text { sum of maximum-height-measure over all consolidation pallets }}{\text { loaded number of consolidation pallets }} .
$$

Here, the maximum-height-measure is calculated in the same way as the corresponding scoring component (34). In Stage 2, "loaded" refers to if feasible placements were found for the empty consolidation pallets. For example, it could be that three consolidated pallets are estimated to accommodate all small packages, while only two of the consolidation pallets fit in the shipping container. (Consolidation pallets must satisfy the minimum possible height constraint, see Table 2 in the paper.)

Fitness 17 (Stages: 2)

The fitness component for maximum possible weight of consolidation pallets based on where they will be placed.

$$
f_{17}(i)=\frac{\text { sum of maximum-weight-measure over all consolidation pallets }}{\text { loaded number of consolidation pallets }} .
$$

Here, the maximum-weight-measure is calculated in the same way as the corresponding scoring component (35). This fitness component is very similar to $(52)$. 
Fitness 18 (Stages: 2)

The fitness component for consolidation pallets closer to the floor is

$f_{18}(i)=\frac{\text { sum of height-from-floor-measure over all consolidation pallets }}{\text { loaded number of consolidation pallets }}$.

Here, the height-from-floor-measure is calculated in the same way as the scoring component (15). This fitness component is also similar to (52).

\section{Comment}

Note that (52)-(54) promote the use of consolidation pallets. For our problem instances (see the Supplementary material data file Input50.csv), the packages in Stage 4 are few and quite small and light. Therefore, promoting the use of consolidation pallets has not caused any difficulties in placing the Stage 4 packages. (Recall that nothing may be stacked on top of a consolidation pallet.) However, with more Stage 4 packages, it would be necessary to be more restrictive in terms of where consolidation pallets are placed, as they limit the placement of Stage 4 packages.

One option for resolving the issue described above would be to not reward lower placements as in (54) but instead reward higher space utilization heightwise, similar to the scoring component in (21). Another option would be to alter scoring component (36) so that consolidation pallets will not always be placed at the points suggested by Stage 2. By placing a filled consolidation pallet somewhere else, it becomes possible to make placements of Stage 4 packages which otherwise would not fit in the shipping container. These kind of simple potential modifications illustrate the flexibility of the algorithm design. 


\subsection{Fitness component weights}

The fixed weights $c_{g}$ applied to the fitness components in the fitness function, see (5) in the paper, are given in Table 3.

Table 3: Fitness component weights for load plan evaluation

\begin{tabular}{cc|l|cccc}
\hline No. Goal & Load plan fitness component & \multicolumn{5}{|c}{ Stage } \\
& & 1 & 2 & 3 & 4 \\
\hline 1 & 1 & Leftover boxes none & 3 & 1 & 3 & 3 \\
2 & 1 & Leftover boxes fewer & 30 & 30 & 30 & 30 \\
3 & 1 & Leftover boxes volume lower & 30 & 30 & 30 & 30 \\
4 & 1 & Leftover boxes weight lower & 30 & 30 & 30 & 30 \\
5 & 2 & Promising spaces for consolidation pallets & 1 & & & \\
6 & 2 & Support behind boxes & 1 & 1 & 1 & 1 \\
7 & 2 & Support in front, left and right of boxes & 1 & 1 & 1 & 1 \\
8 & 2 & Utilization of floor space higher & 1 & 1 & 1 & \\
9 & 2 & Support for box not on floor - behind non-slim & 1 & 1 & & 1 \\
& & box, and behind and in front of slim box & & & & \\
10 & 3 & Center of gravity lengthwise closer to center & 1 & & & \\
11 & 3 & Center of gravity widthwise closer to center & 1 & & & \\
12 & 3 & Center of gravity heightwise closer to floor & 1 & & & \\
13 & 4 & Boxes with pallet not rotated & 1 & 1 & & 1 \\
14 & 4 & Boxes with pallet reachable by forklift & 1 & 1 & & 1 \\
15 & 4 & Boxes without pallet not on floor & \multicolumn{5}{|c|}{5} \\
\hline 16 & 2 & Consolidation pallets possible height & & & \\
17 & 2 & Consolidation pallets possible weight & 15 & & \\
18 & 2 & Consolidation pallets closer to floor & \multicolumn{5}{|c|}{13} & & \\
\hline
\end{tabular}




\section{Tuning of the scoring weights}

In our algorithm, each scoring component $j$ is given a weight $w_{j} \in[0, \bar{w}]$, where $\bar{w}=10000$. Here, we present Stage 1 scoring weights for the best load plan found using box plots for each problem instance over several runs (since GA is stochastic), and an aggregated box plot over all runs, which illustrates the variation of the scoring weights. We made ten runs for each problem instance, giving in total $50 \times 10=500$ runs. The results are from Stage 1 since it is by far the most crucial and challenging stage, as discussed in Section 4 of the paper. Weights for components in $j \in J_{1}$ and $j \in J_{2}$ are shown to the left (light gray) and to the right (darker gray), respectively. The figures support the observations made in Section 4.1 of the paper.

\section{Scoring weights for each problem instance}

Figures 958 show the distribution of the scoring weights which gave the best load plans over ten runs for each problem, with 200 GA generations in each run. Note that for each of the 20 weights, there are examples of problem instances where the weight is tuned to a tight range, as well as instances where the weight varies widely. For example, scoring weight 1 has a tight high range for Problem 12, a tight low range for Problem 14, and a very wide range with the mean close to $\bar{w} / 2$ for Problem 11. The interpretation is that the corresponding property "box with higher weight closer to floor" is more important for finding a good load plan for Problem 12 and 14 than it is for Problem 11. Even though intuition or common sense may suggest that this property should always be desirable, Problem 14 and several other instances demonstrate that it can be necessary to make trade-offs between conflicting load plan properties. The fact that a property is important for an instance only means that the value of the corresponding scoring weight should fall within a tight range, but not necessarily within a high range. 


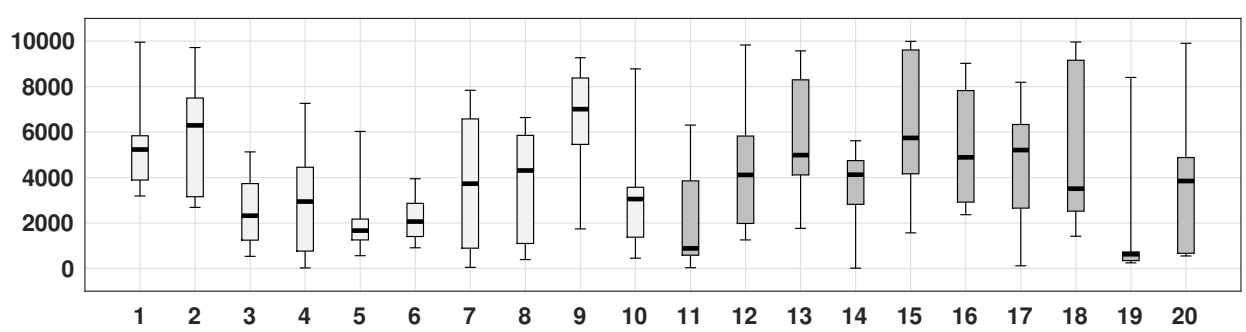

Figure 9: Scoring weight values for Problem 1

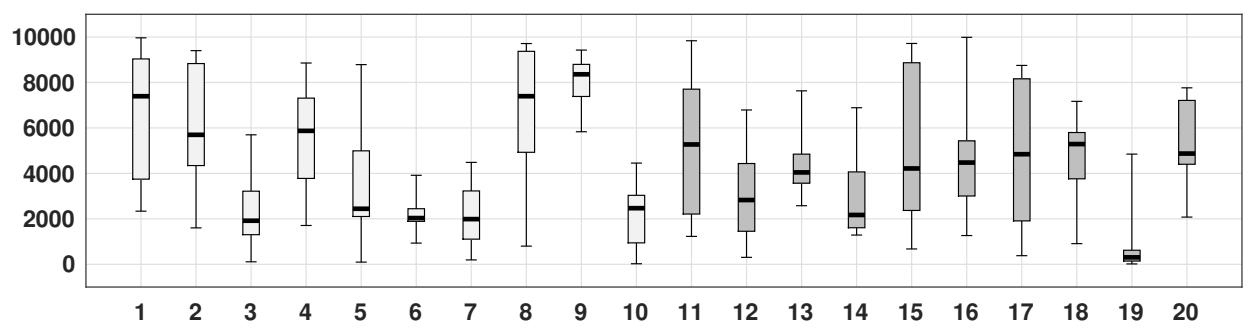

Figure 10: Scoring weight values for Problem 2

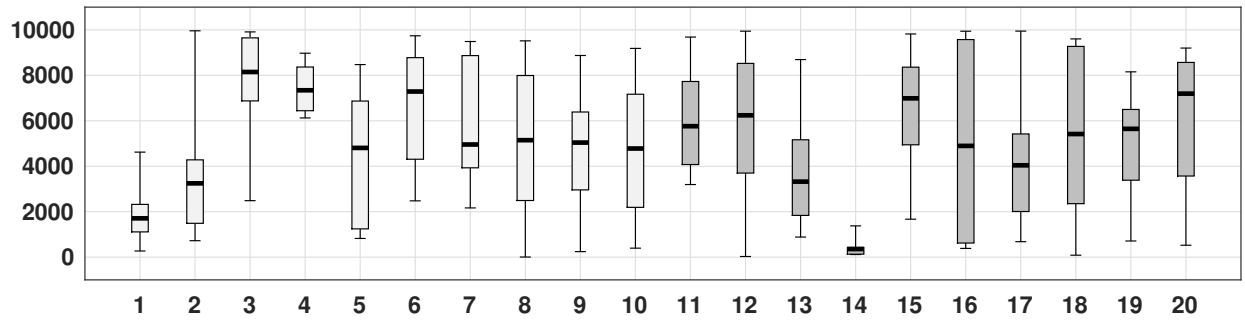

Figure 11: Scoring weight values for Problem 3

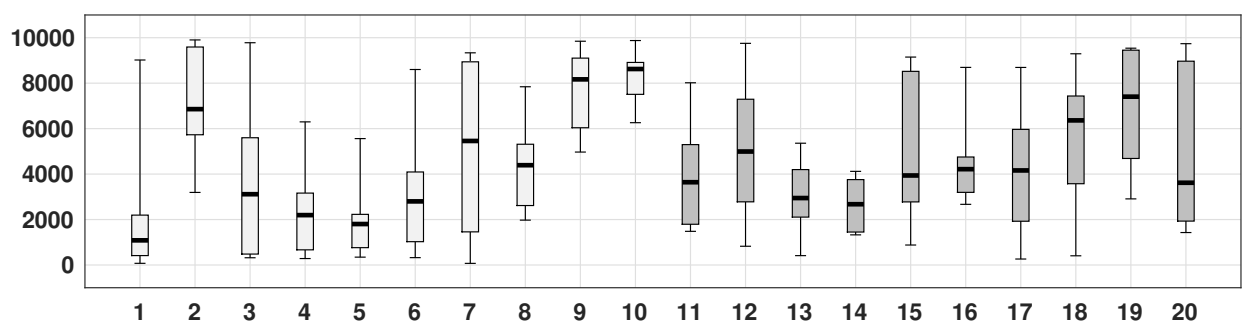

Figure 12: Scoring weight values for Problem 4

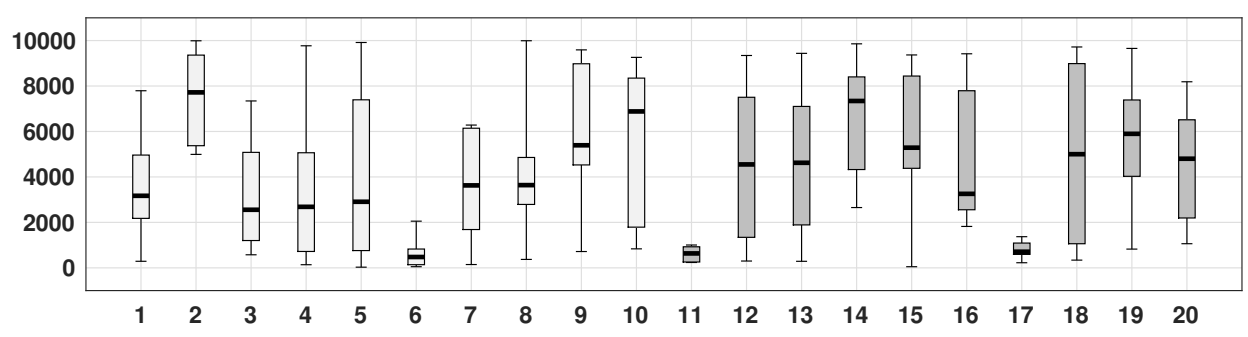

Figure 13: Scoring weight values for Problem 5 


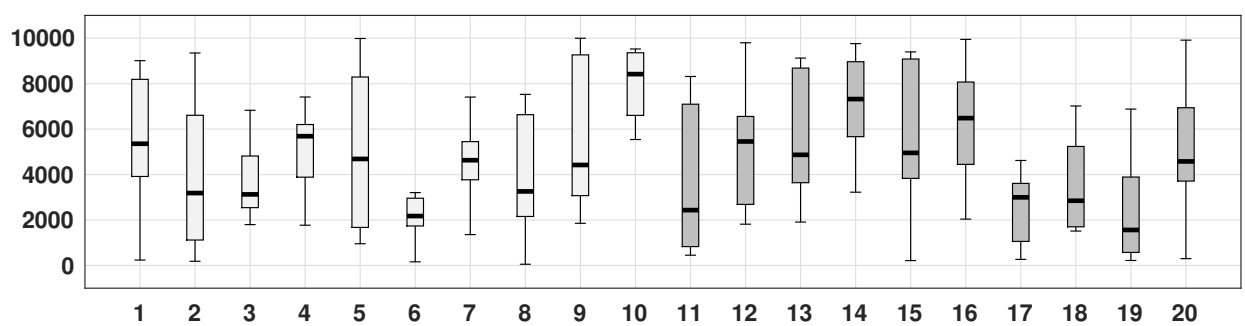

Figure 14: Scoring weight values for Problem 6

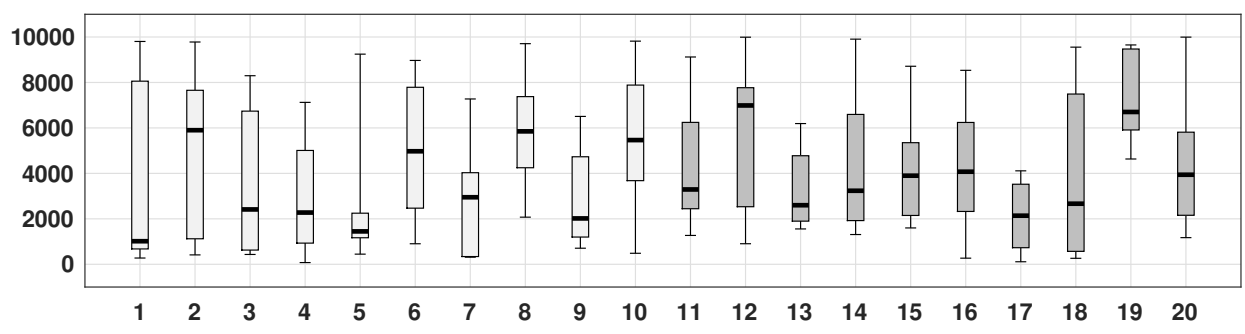

Figure 15: Scoring weight values for Problem 7

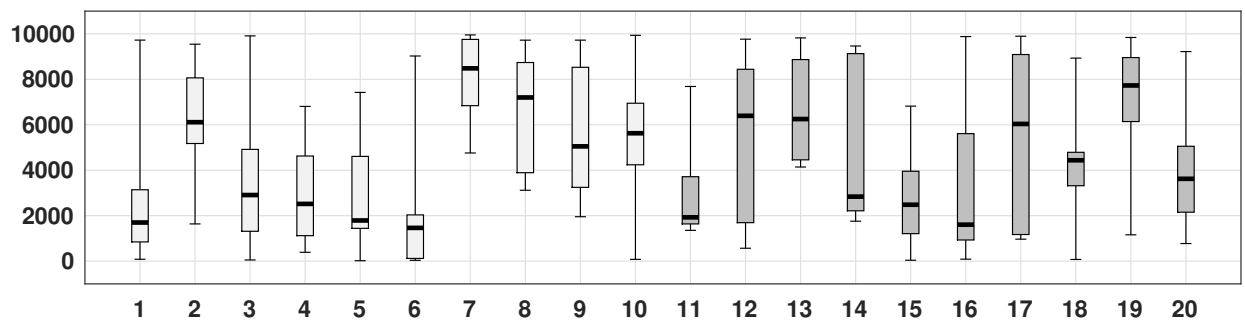

Figure 16: Scoring weight values for Problem 8

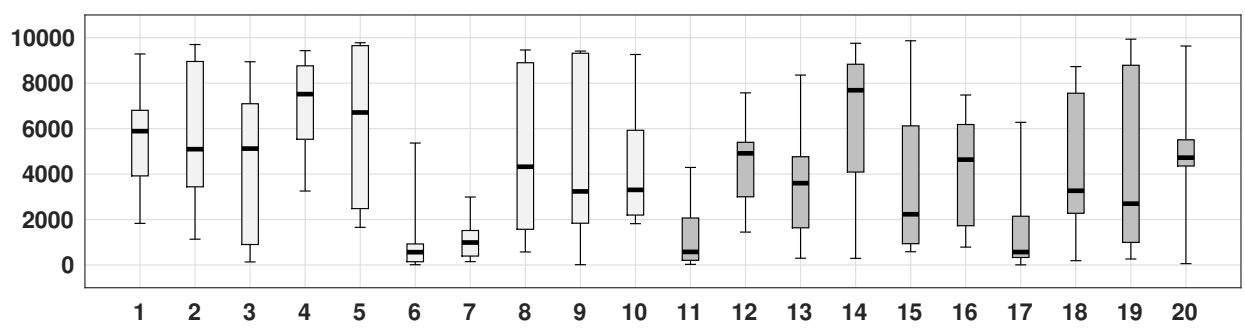

Figure 17: Scoring weight values for Problem 9

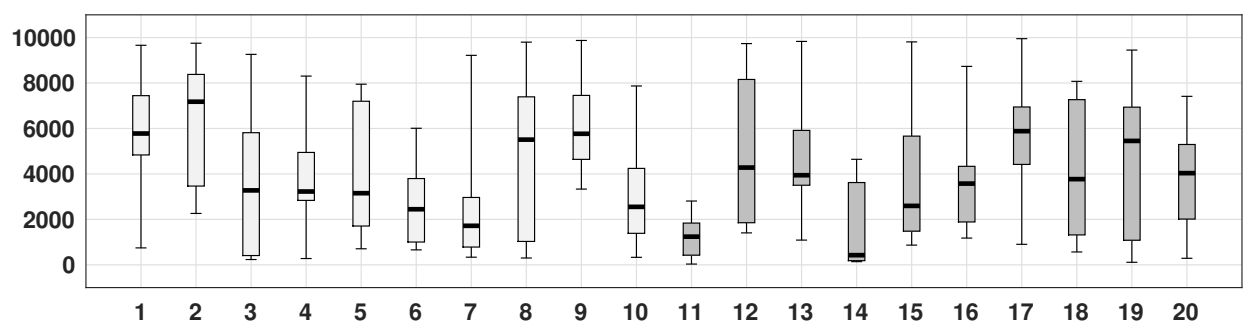

Figure 18: Scoring weight values for Problem 10 


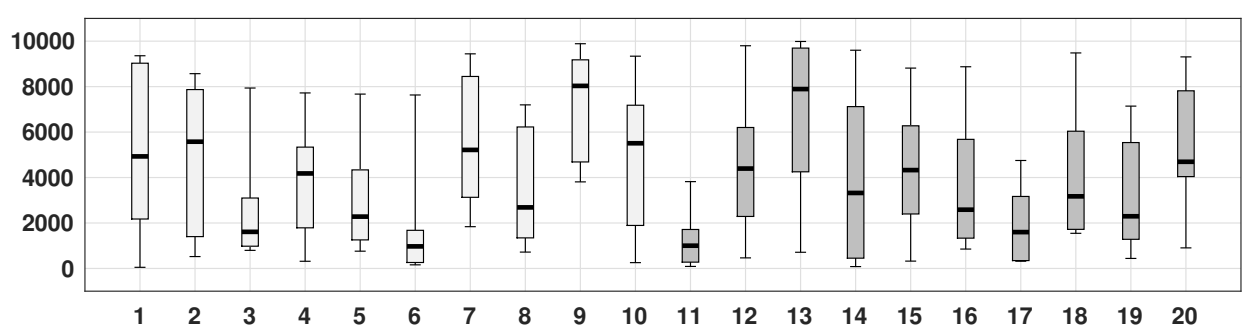

Figure 19: Scoring weight values for Problem 11

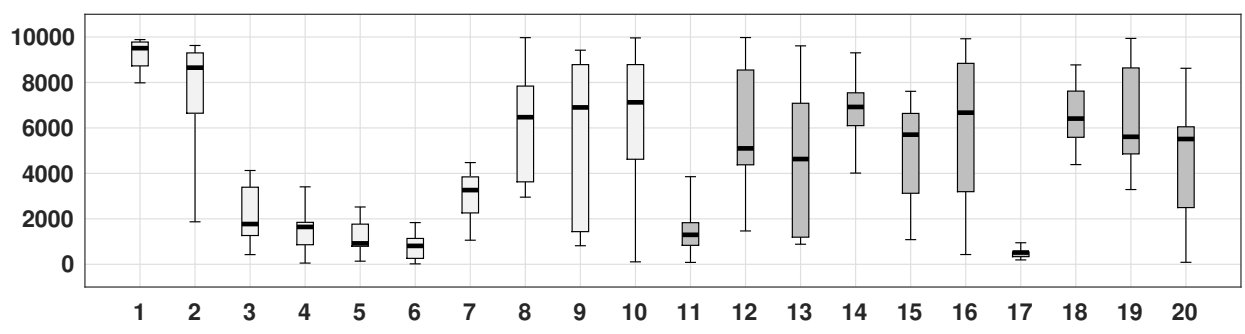

Figure 20: Scoring weight values for Problem 12

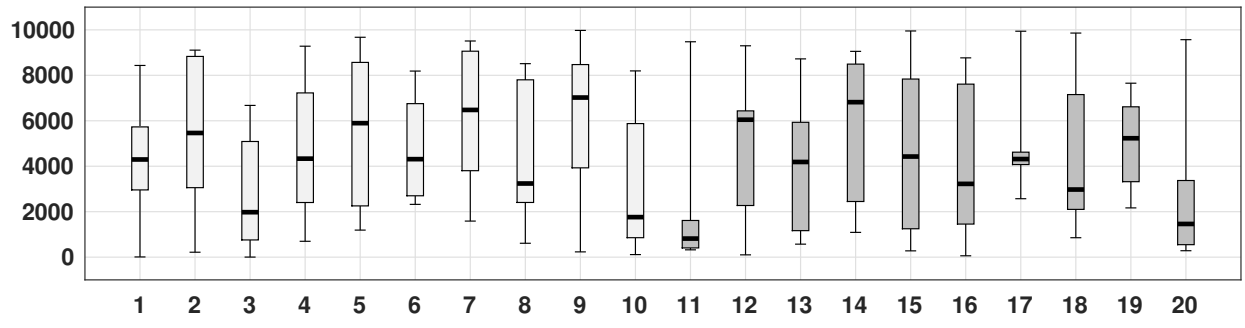

Figure 21: Scoring weight values for Problem 13

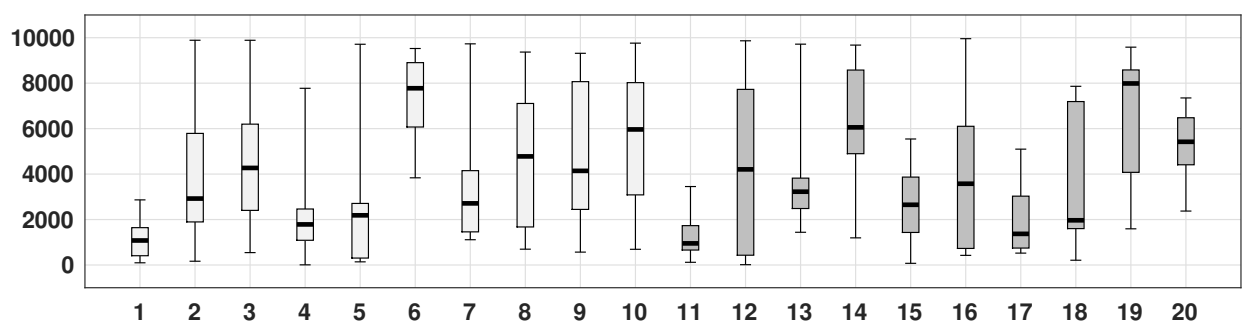

Figure 22: Scoring weight values for Problem 14

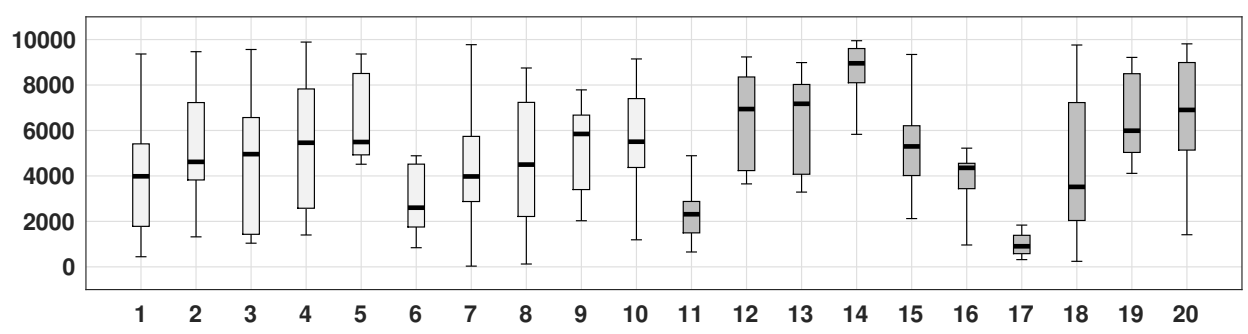

Figure 23: Scoring weight values for Problem 15 


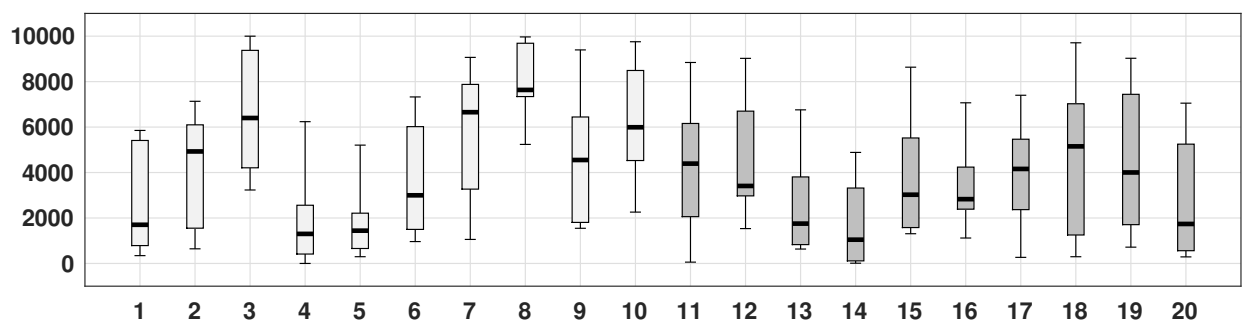

Figure 24: Scoring weight values for Problem 16

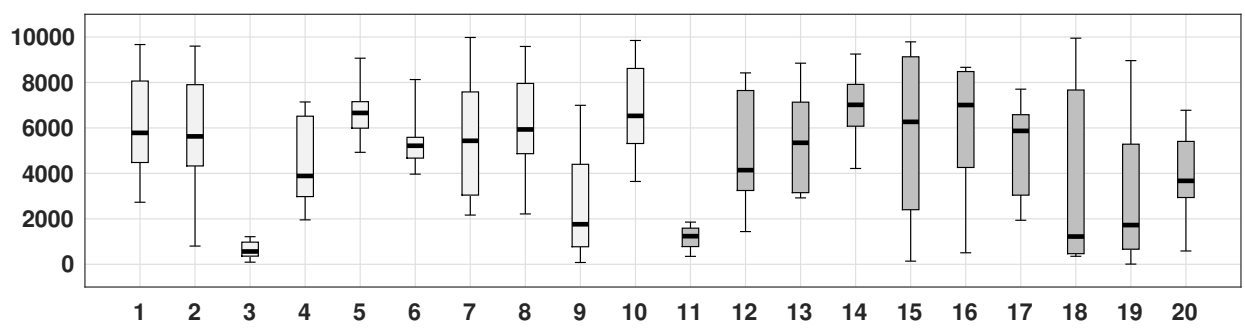

Figure 25: Scoring weight values for Problem 17

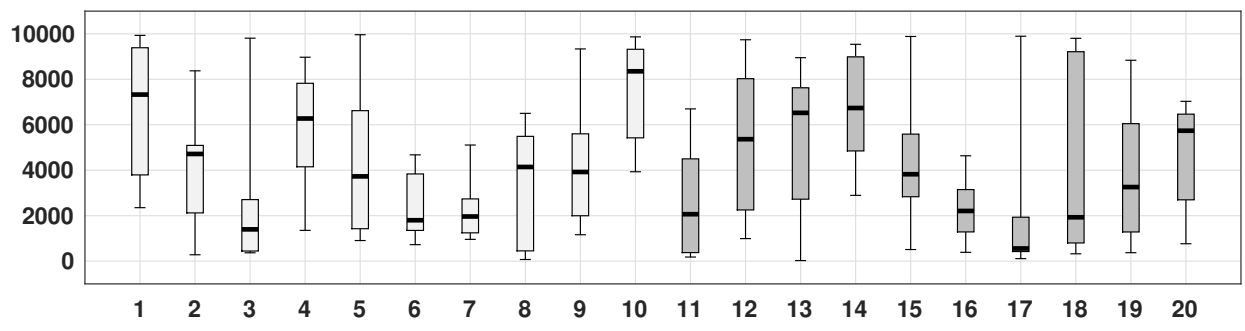

Figure 26: Scoring weight values for Problem 18

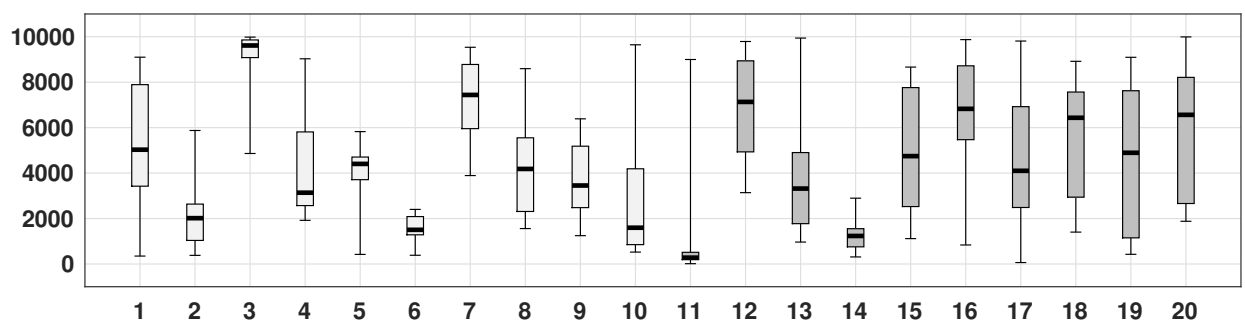

Figure 27: Scoring weight values for Problem 19

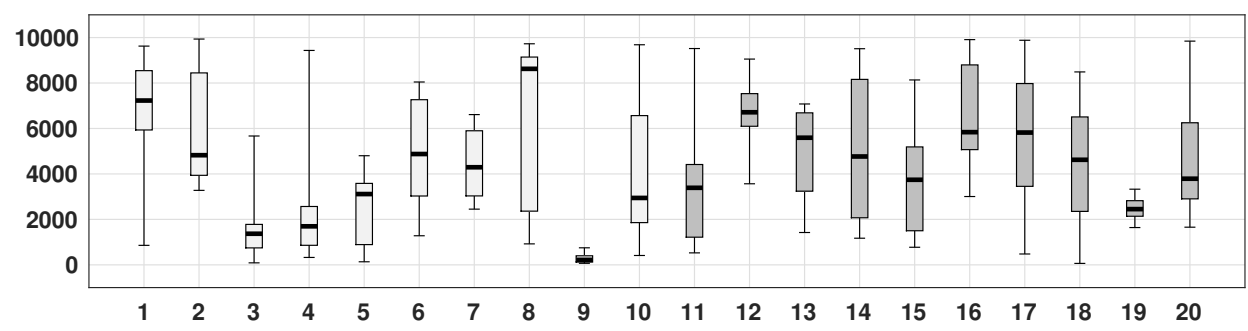

Figure 28: Scoring weight values for Problem 20 


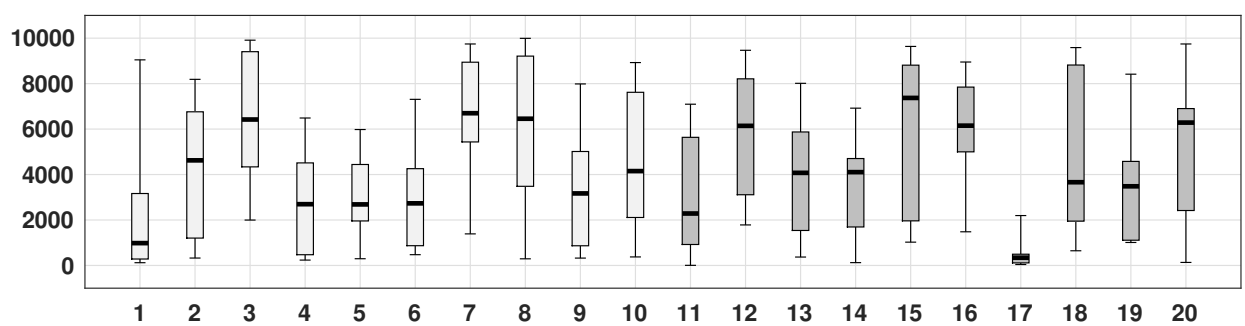

Figure 29: Scoring weight values for Problem 21

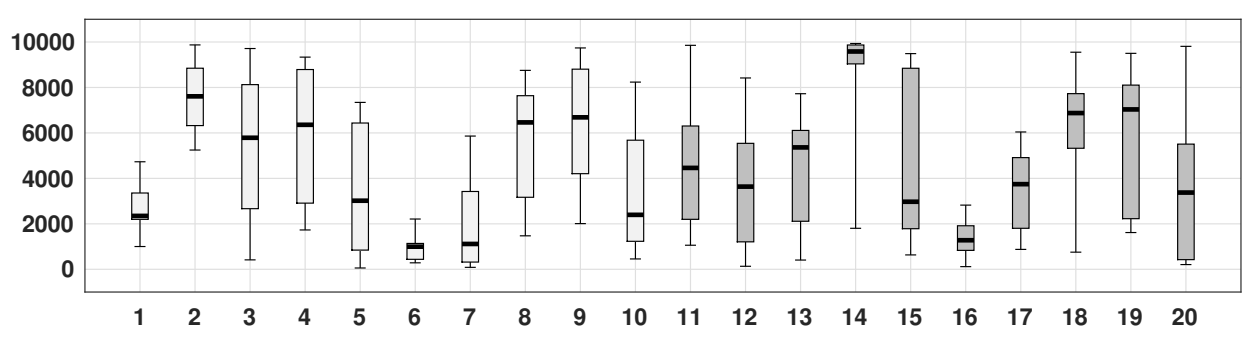

Figure 30: Scoring weight values for Problem 22

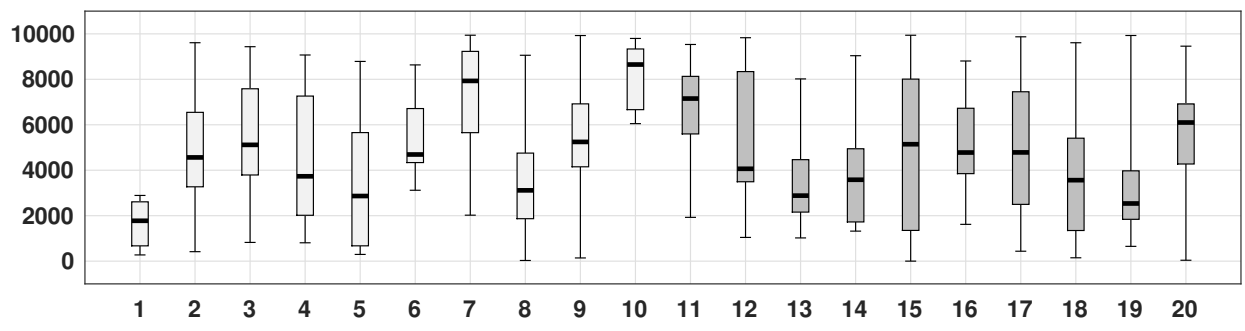

Figure 31: Scoring weight values for Problem 23

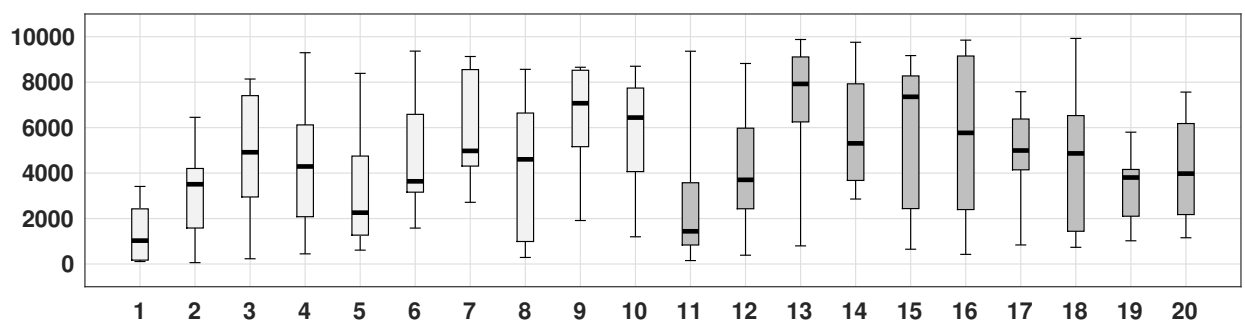

Figure 32: Scoring weight values for Problem 24

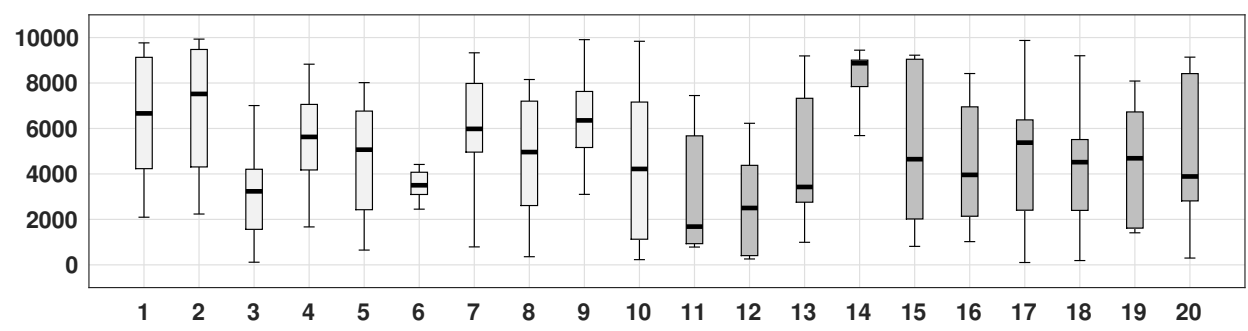

Figure 33: Scoring weight values for Problem 25 


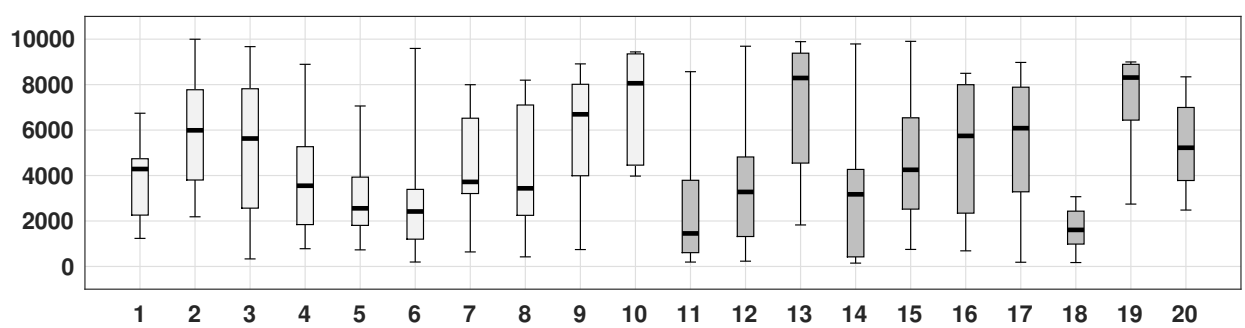

Figure 34: Scoring weight values for Problem 26

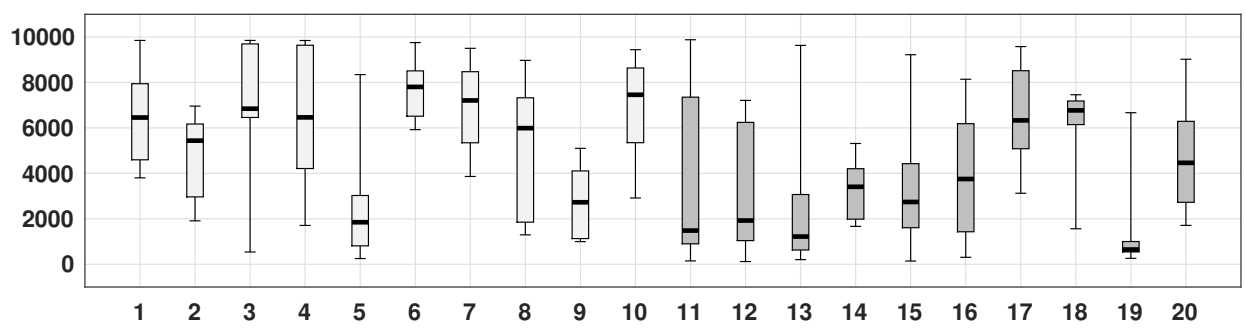

Figure 35: Scoring weight values for Problem 27

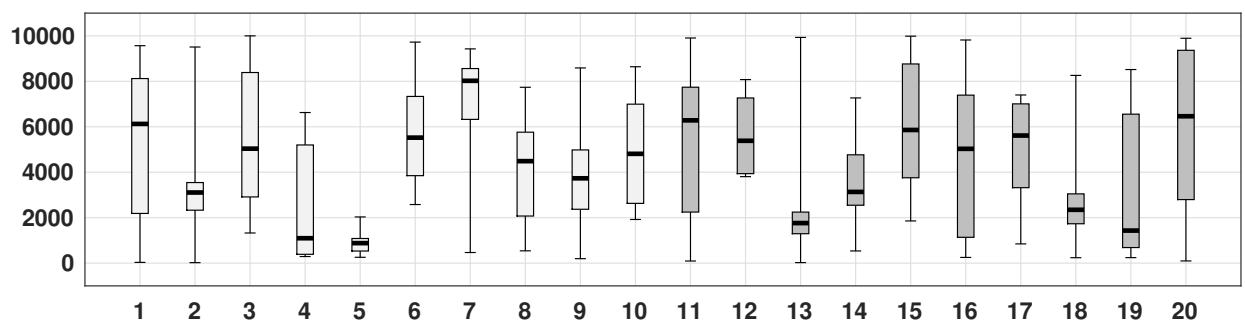

Figure 36: Scoring weight values for Problem 28

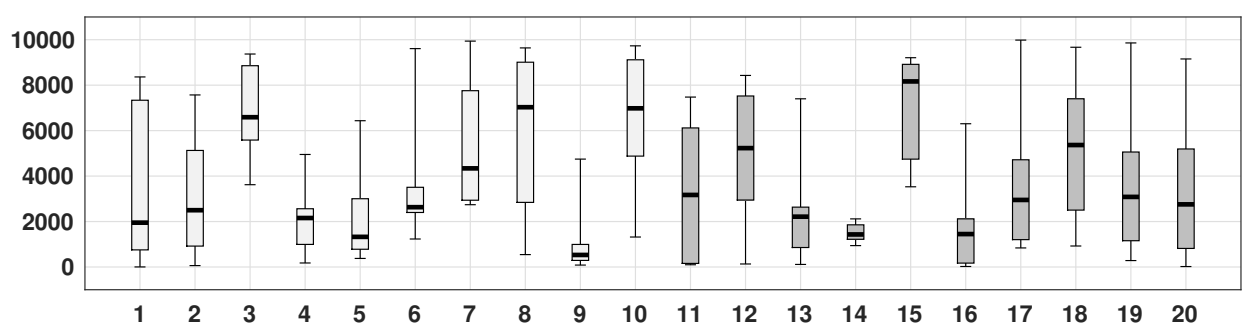

Figure 37: Scoring weight values for Problem 29

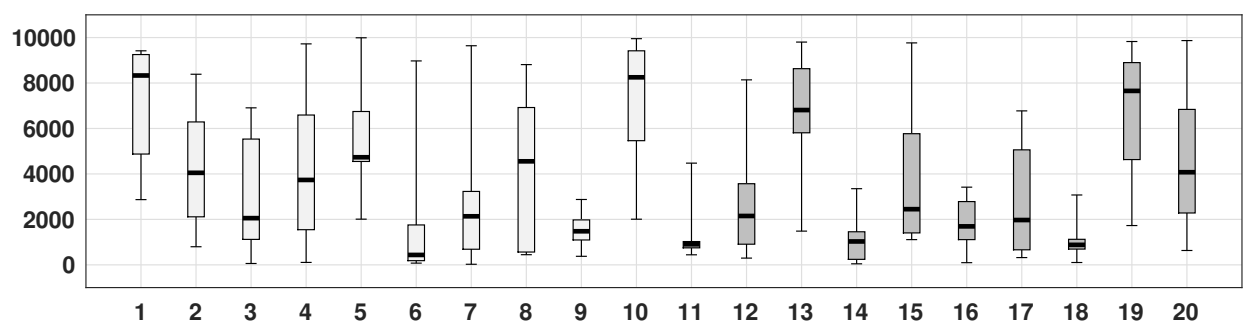

Figure 38: Scoring weight values for Problem 30 


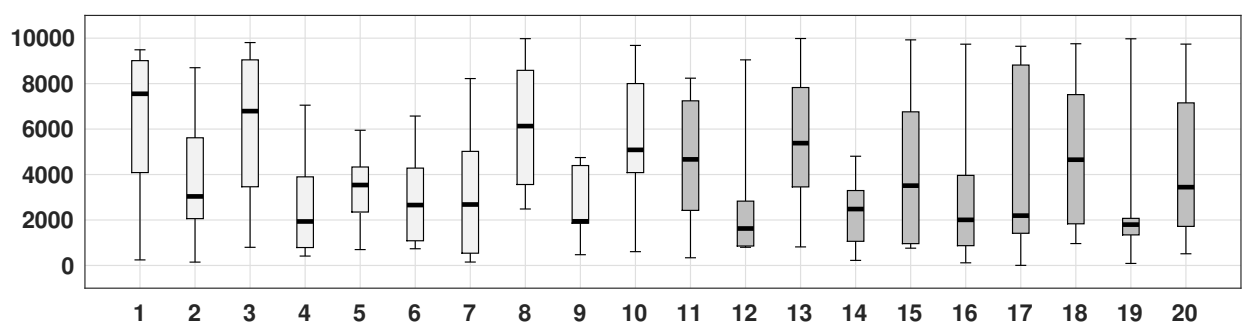

Figure 39: Scoring weight values for Problem 31

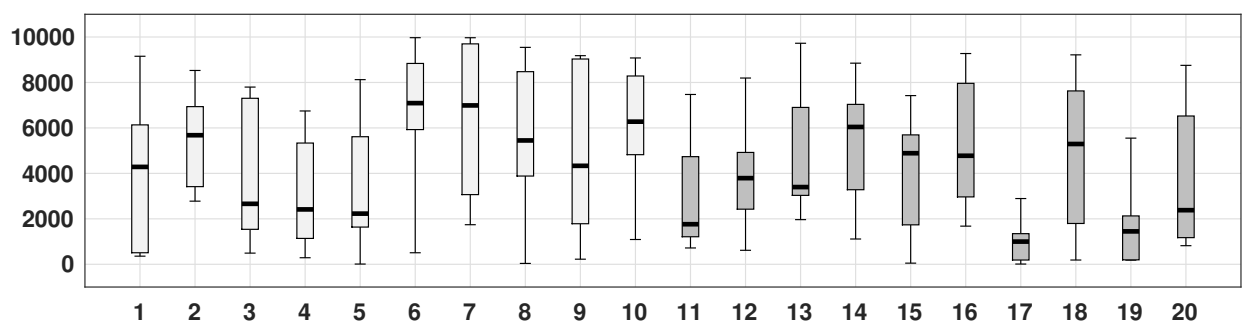

Figure 40: Scoring weight values for Problem 32

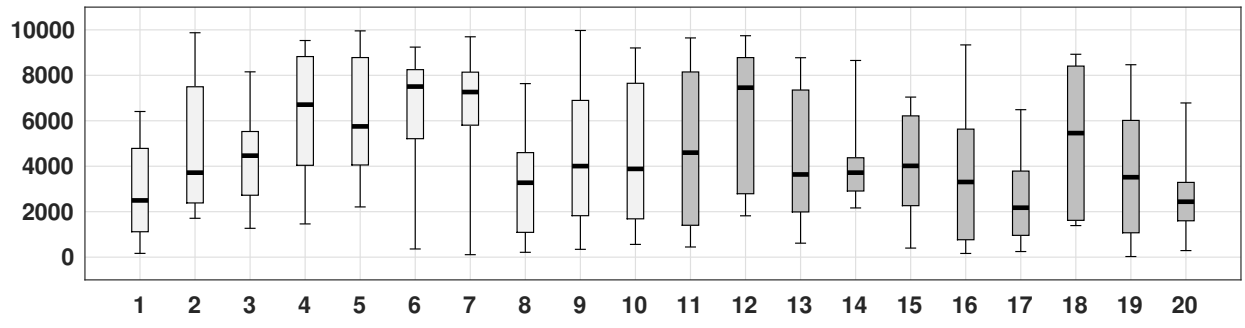

Figure 41: Scoring weight values for Problem 33

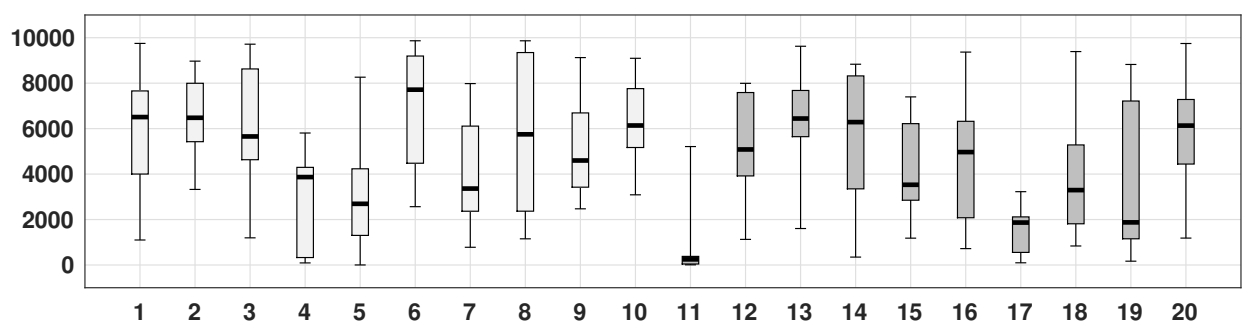

Figure 42: Scoring weight values for Problem 34

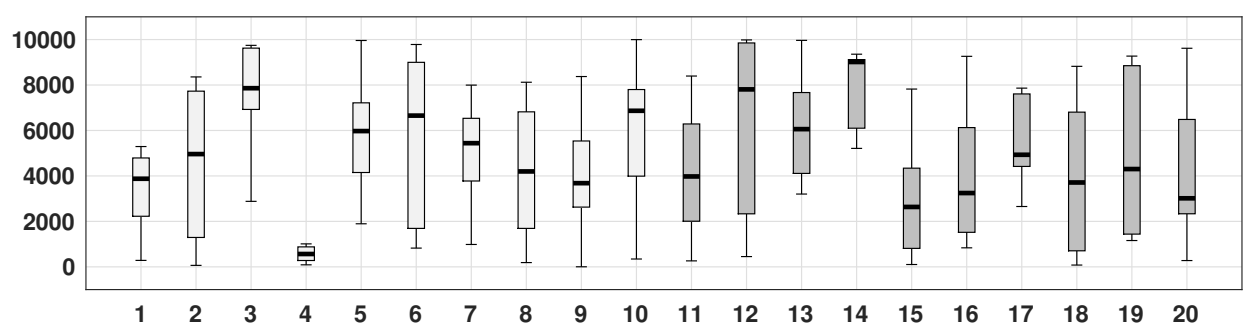

Figure 43: Scoring weight values for Problem 35 


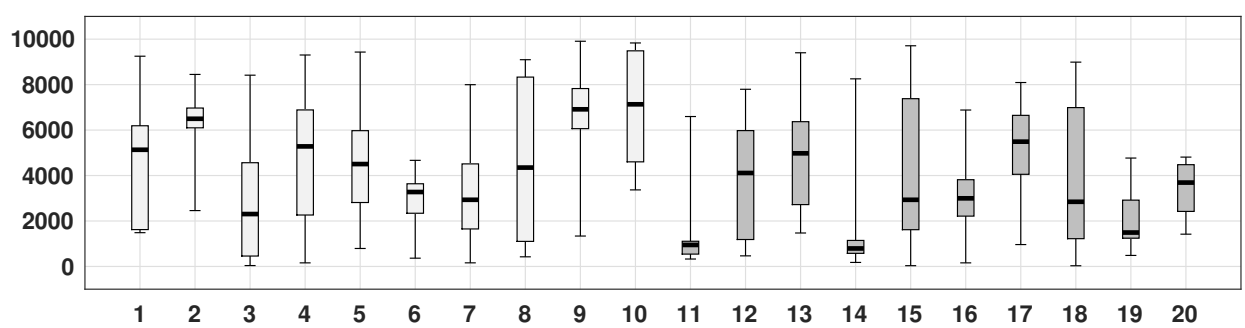

Figure 44: Scoring weight values for Problem 36

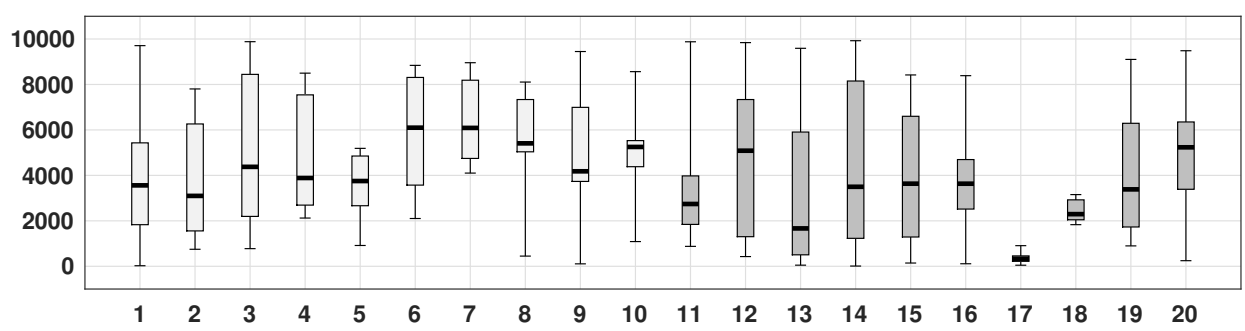

Figure 45: Scoring weight values for Problem 37

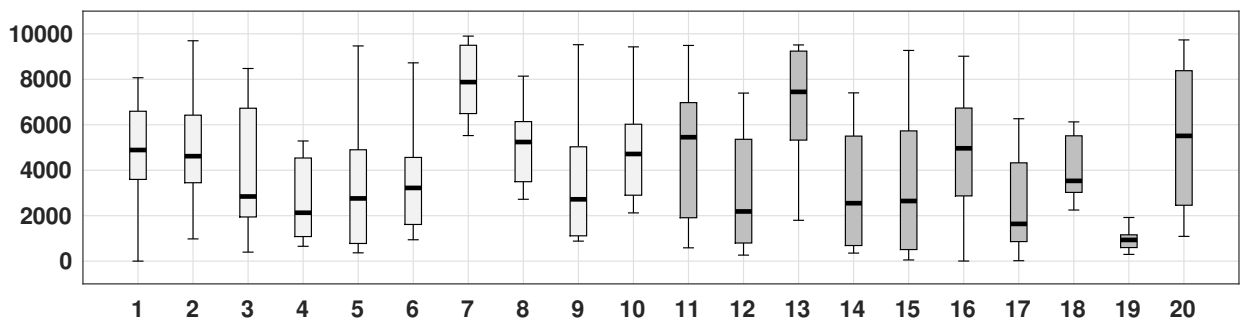

Figure 46: Scoring weight values for Problem 38

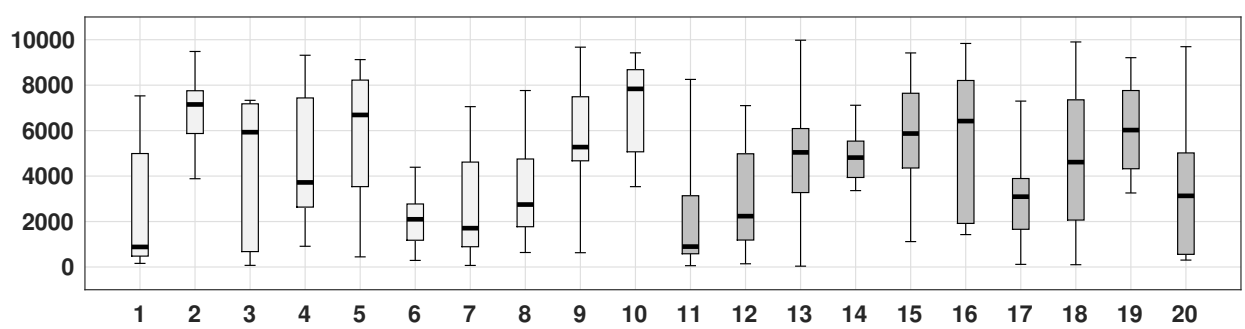

Figure 47: Scoring weight values for Problem 39

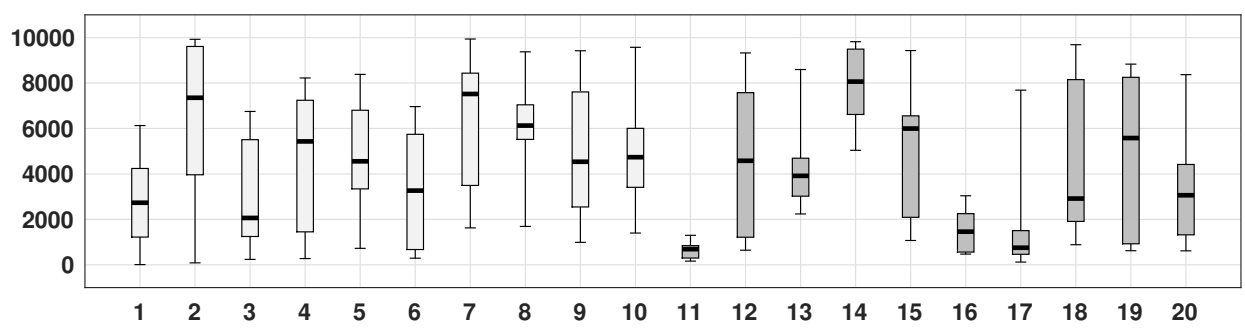

Figure 48: Scoring weight values for Problem 40 


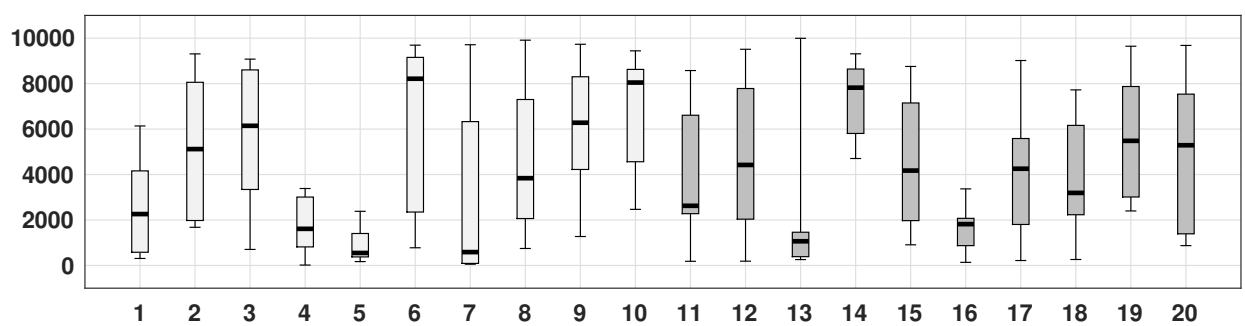

Figure 49: Scoring weight values for Problem 41

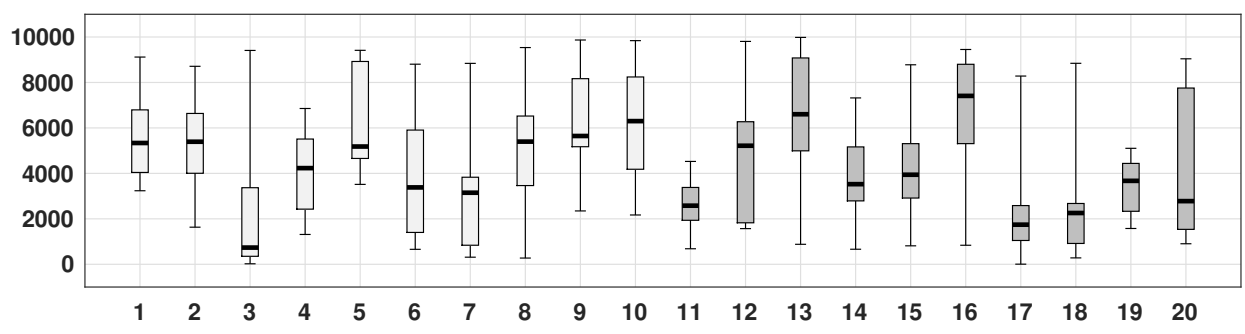

Figure 50: Scoring weight values for Problem 42

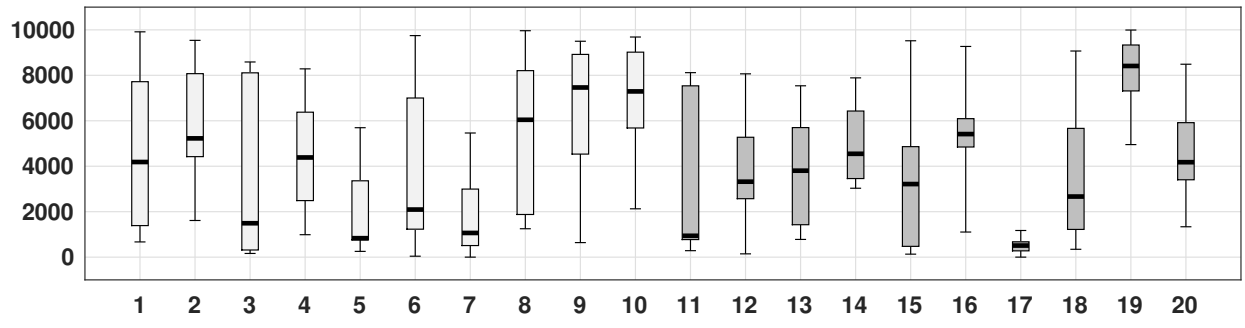

Figure 51: Scoring weight values for Problem 43

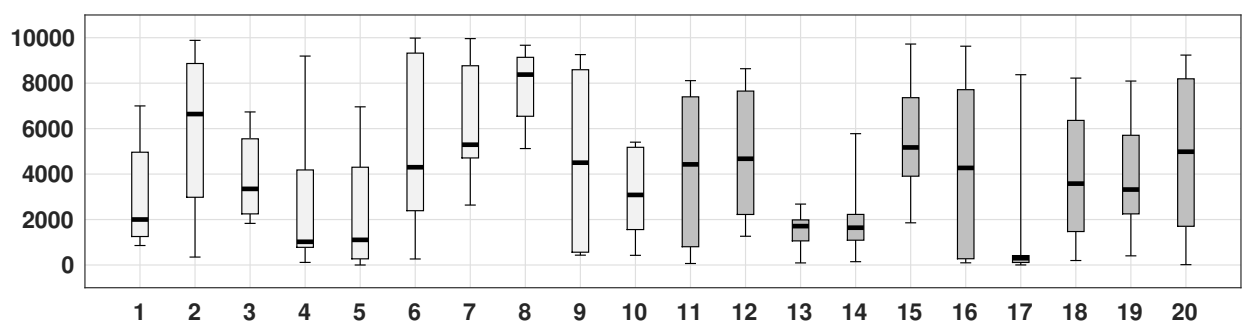

Figure 52: Scoring weight values for Problem 44

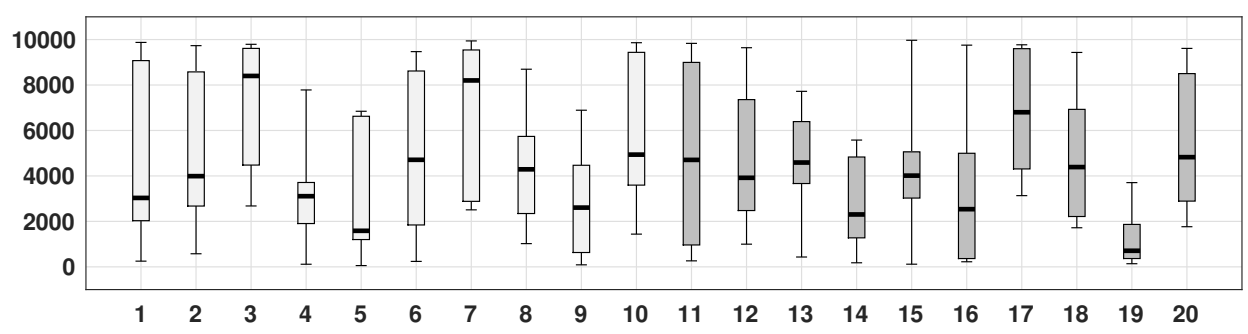

Figure 53: Scoring weight values for Problem 45 


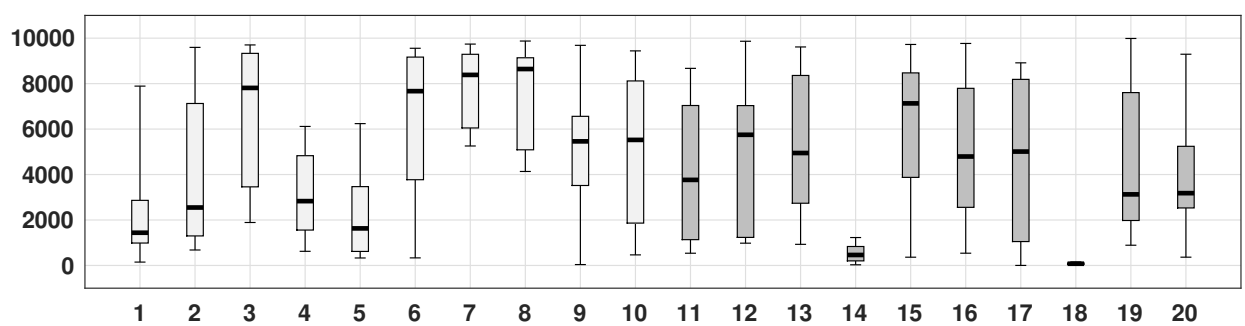

Figure 54: Scoring weight values for Problem 46

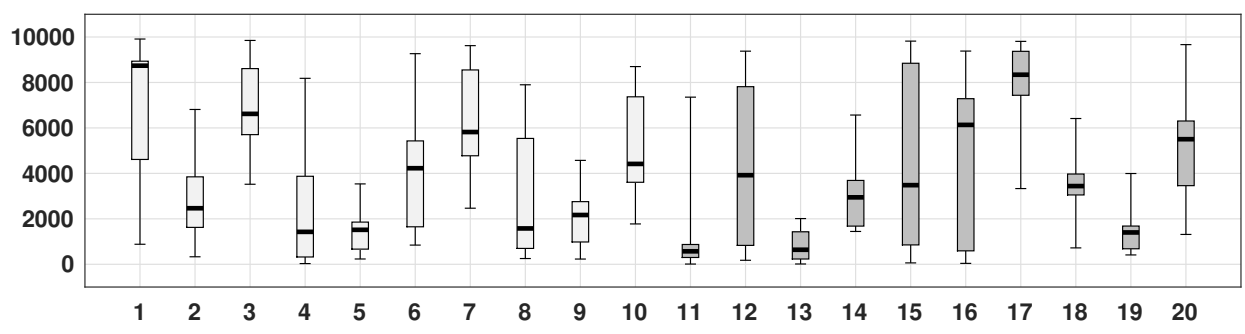

Figure 55: Scoring weight values for Problem 47

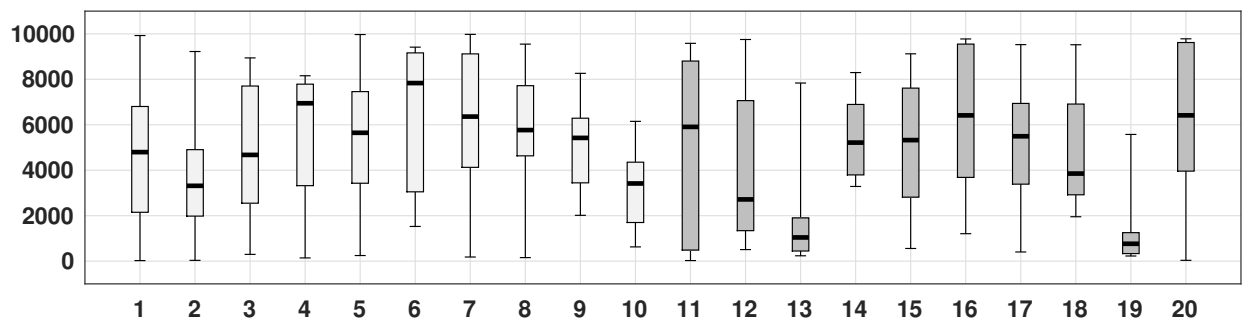

Figure 56: Scoring weight values for Problem 48

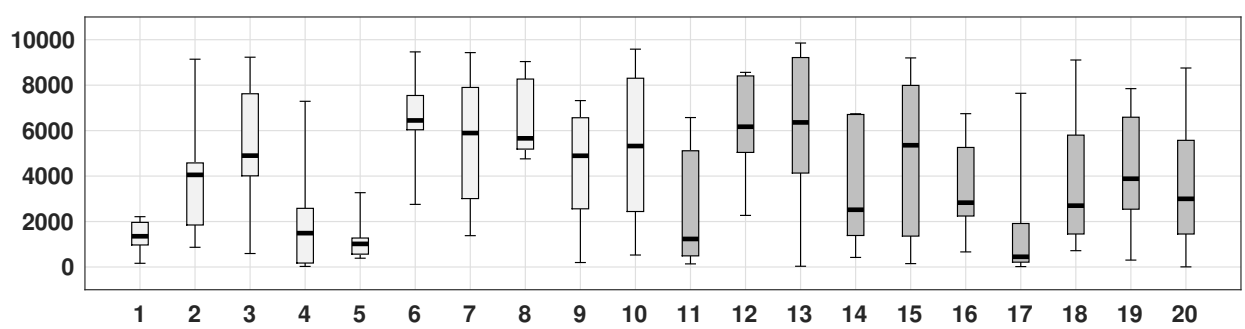

Figure 57: Scoring weight values for Problem 49

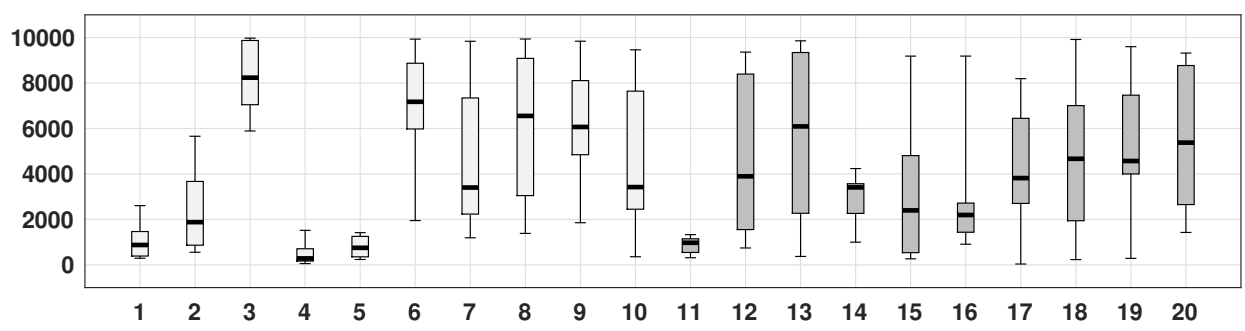

Figure 58: Scoring weight values for Problem 50 
Scoring weights aggregated for all problem instances

Figure 59 shows the distribution of the scoring weight values producing the best load plans over ten runs, aggregated for all 50 problem instances. Note that on an aggregated level, each of the 20 weights has a very wide range. For example, we may compare the range of scoring weight 1 on an aggregated level in this figure with the ranges for Problem 12 and 14 in Figure 20 and 22 , respectively. The very wide range of the scoring weights on an aggregated level confirms the need of instance dependent settings. Because of the multiple goals for loading and the heterogeneity of problem instances, the self-adaptiveness of our approach is indeed needed to guide the placement heuristic to find good load plans.

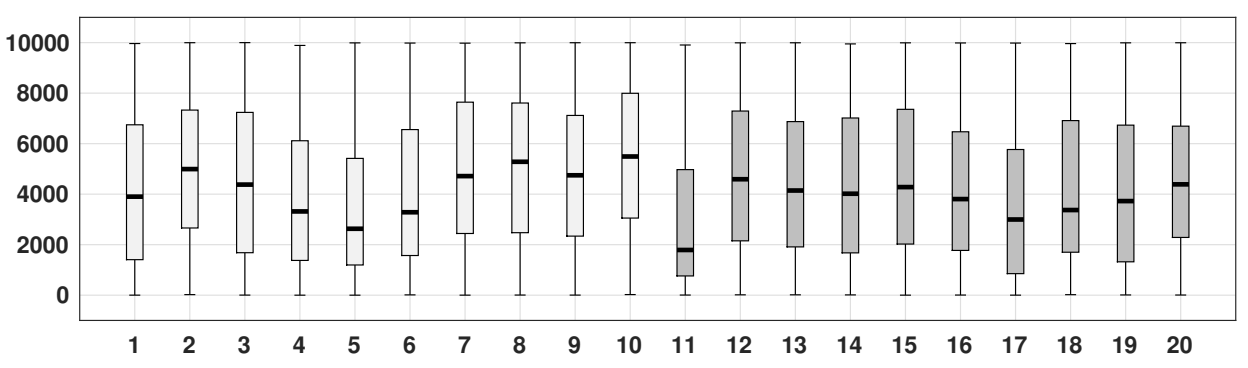

Figure 59: Aggregated scoring weight values for all 50 problem instances

\section{Results for algorithm versions}

We present the detailed information behind Table 6 of the paper, where the 22 algorithm versions are compared. In total, $50 \cdot(11+11 \cdot 10)=6050$ runs were made. Note that versions 1-11 need only one run each since they are deterministic, while versions $12-22$, which are non-deterministic, were run 10 times for each problem instance. Total run time on the Dell laptop with an Intel Core i5-2520M 2.50 GHz CPU and 4 GB RAM (see Section 4 of the paper) is estimated to 2500 hours, that is, 3.5 months. 
Table 4: Stage 1 results for 22 versions in terms of fitting all packages and average fitness value (\%) for the runs where all packages fit. $\mathrm{T}=$ Tuning of the scoring weights (Yes/No); $\mathrm{C}=$ Components in the scoring function; $\mathrm{A}=$ Adjusted duplicate points used (Yes/No); Sum $=$ Sum of success rates of fitting all packages; Versions 1-11.

\begin{tabular}{|c|c|c|c|c|c|c|c|c|c|c|c|}
\hline Ver. & 1 & 2 & 3 & 4 & 5 & 6 & 7 & 8 & 9 & 10 & 11 \\
\hline $\mathrm{T}$ & $\mathrm{N}$ & $\mathrm{N}$ & $\mathrm{N}$ & $\mathrm{N}$ & $\mathrm{N}$ & $\mathrm{N}$ & $\mathrm{N}$ & $\mathrm{N}$ & $\mathrm{N}$ & $\mathrm{N}$ & $\mathrm{N}$ \\
\hline C & $3 \mathrm{HA}$ & $3 \mathrm{HA}$ & $3 \mathrm{AH}$ & $3 \mathrm{AH}$ & $9+9$ & $9+9$ & 10 & 10 & 18 & 18 & 20 \\
\hline A & $\mathrm{N}$ & $\mathrm{Y}$ & $\mathrm{N}$ & Y & $\mathrm{N}$ & $\mathrm{Y}$ & $\mathrm{N}$ & Y & $\mathrm{N}$ & Y & Y \\
\hline 1 & 1.0 & 1.0 & 1.0 & 1.0 & 1.0 & 1.0 & 1.0 & 1.0 & 0.0 & 1.0 & 1.0 \\
\hline 2 & 0.0 & 0.0 & 1.0 & 1.0 & 1.0 & 1.0 & 1.0 & 1.0 & 1.0 & 1.0 & 1.0 \\
\hline 3 & 1.0 & 1.0 & 1.0 & 1.0 & 1.0 & 1.0 & 0.0 & 0.0 & 0.0 & 0.0 & 1.0 \\
\hline 4 & 1.0 & 1.0 & 1.0 & 1.0 & 1.0 & 1.0 & 1.0 & 1.0 & 1.0 & 1.0 & 1.0 \\
\hline 5 & 1.0 & 1.0 & 1.0 & 1.0 & 1.0 & 1.0 & 1.0 & 1.0 & 1.0 & 1.0 & 1.0 \\
\hline 6 & 0.0 & 0.0 & 0.0 & 0.0 & 0.0 & 0.0 & 0.0 & 0.0 & 0.0 & 0.0 & 0.0 \\
\hline 7 & 1.0 & 1.0 & 1.0 & 1.0 & 1.0 & 1.0 & 1.0 & 1.0 & 0.0 & 0.0 & 0.0 \\
\hline 8 & 0.0 & 0.0 & 0.0 & 0.0 & 0.0 & 0.0 & 0.0 & 0.0 & 0.0 & 0.0 & 0.0 \\
\hline 9 & 1.0 & 1.0 & 1.0 & 1.0 & 1.0 & 1.0 & 1.0 & 1.0 & 0.0 & 0.0 & 0.0 \\
\hline 10 & 1.0 & 1.0 & 1.0 & 1.0 & 1.0 & 1.0 & 1.0 & 1.0 & 0.0 & 0.0 & 1.0 \\
\hline 11 & 1.0 & 1.0 & 1.0 & 1.0 & 1.0 & 1.0 & 1.0 & 1.0 & 0.0 & 0.0 & 0.0 \\
\hline 12 & 0.0 & 0.0 & 0.0 & 0.0 & 0.0 & 0.0 & 0.0 & 0.0 & 0.0 & 0.0 & 0.0 \\
\hline 13 & 0.0 & 0.0 & 1.0 & 1.0 & 1.0 & 1.0 & 1.0 & 1.0 & 1.0 & 1.0 & 1.0 \\
\hline 14 & 0.0 & 0.0 & 1.0 & 1.0 & 0.0 & 0.0 & 0.0 & 0.0 & 0.0 & 0.0 & 0.0 \\
\hline 15 & 1.0 & 1.0 & 1.0 & 1.0 & 1.0 & 1.0 & 1.0 & 1.0 & 1.0 & 1.0 & 1.0 \\
\hline 16 & 0.0 & 0.0 & 0.0 & 0.0 & 0.0 & 0.0 & 0.0 & 0.0 & 0.0 & 0.0 & 0.0 \\
\hline 17 & 0.0 & 0.0 & 0.0 & 0.0 & 1.0 & 0.0 & 1.0 & 1.0 & 1.0 & 1.0 & 1.0 \\
\hline 18 & 1.0 & 1.0 & 0.0 & 0.0 & 0.0 & 0.0 & 1.0 & 1.0 & 0.0 & 0.0 & 0.0 \\
\hline 19 & 0.0 & 0.0 & 0.0 & 0.0 & 0.0 & 0.0 & 0.0 & 0.0 & 0.0 & 0.0 & 0.0 \\
\hline 20 & 0.0 & 0.0 & 0.0 & 0.0 & 0.0 & 0.0 & 0.0 & 0.0 & 0.0 & 0.0 & 0.0 \\
\hline 21 & 0.0 & 0.0 & 1.0 & 1.0 & 1.0 & 1.0 & 1.0 & 1.0 & 1.0 & 1.0 & 0.0 \\
\hline 22 & 1.0 & 1.0 & 1.0 & 1.0 & 1.0 & 1.0 & 1.0 & 1.0 & 1.0 & 1.0 & 1.0 \\
\hline 23 & 0.0 & 0.0 & 1.0 & 1.0 & 1.0 & 1.0 & 1.0 & 1.0 & 0.0 & 0.0 & 0.0 \\
\hline 24 & 0.0 & 0.0 & 0.0 & 0.0 & 0.0 & 0.0 & 0.0 & 0.0 & 0.0 & 0.0 & 0.0 \\
\hline 25 & 0.0 & 0.0 & 1.0 & 1.0 & 1.0 & 1.0 & 1.0 & 1.0 & 1.0 & 1.0 & 1.0 \\
\hline 26 & 0.0 & 0.0 & 1.0 & 1.0 & 1.0 & 0.0 & 0.0 & 0.0 & 1.0 & 0.0 & 0.0 \\
\hline 27 & 0.0 & 0.0 & 0.0 & 0.0 & 0.0 & 0.0 & 0.0 & 0.0 & 0.0 & 0.0 & 0.0 \\
\hline 28 & 1.0 & 1.0 & 1.0 & 1.0 & 1.0 & 1.0 & 1.0 & 1.0 & 0.0 & 0.0 & 0.0 \\
\hline 29 & 0.0 & 0.0 & 0.0 & 0.0 & 0.0 & 0.0 & 0.0 & 0.0 & 0.0 & 0.0 & 0.0 \\
\hline 30 & 0.0 & 0.0 & 0.0 & 0.0 & 0.0 & 0.0 & 0.0 & 0.0 & 0.0 & 0.0 & 0.0 \\
\hline 31 & 0.0 & 0.0 & 1.0 & 1.0 & 0.0 & 0.0 & 0.0 & 0.0 & 0.0 & 0.0 & 0.0 \\
\hline 32 & 0.0 & 0.0 & 1.0 & 1.0 & 1.0 & 0.0 & 0.0 & 0.0 & 0.0 & 0.0 & 0.0 \\
\hline 33 & 1.0 & 1.0 & 1.0 & 1.0 & 1.0 & 1.0 & 0.0 & 0.0 & 0.0 & 1.0 & 1.0 \\
\hline 34 & 0.0 & 0.0 & 1.0 & 1.0 & 0.0 & 0.0 & 0.0 & 0.0 & 0.0 & 0.0 & 0.0 \\
\hline 35 & 0.0 & 0.0 & 0.0 & 0.0 & 0.0 & 0.0 & 0.0 & 0.0 & 0.0 & 0.0 & 0.0 \\
\hline 36 & 1.0 & 1.0 & 1.0 & 1.0 & 1.0 & 1.0 & 1.0 & 1.0 & 0.0 & 0.0 & 0.0 \\
\hline 37 & 0.0 & 0.0 & 1.0 & 1.0 & 0.0 & 0.0 & 0.0 & 0.0 & 0.0 & 0.0 & 0.0 \\
\hline 38 & 0.0 & 0.0 & 0.0 & 0.0 & 0.0 & 0.0 & 0.0 & 0.0 & 0.0 & 0.0 & 0.0 \\
\hline 39 & 1.0 & 1.0 & 1.0 & 1.0 & 1.0 & 1.0 & 1.0 & 1.0 & 1.0 & 1.0 & 1.0 \\
\hline 40 & 1.0 & 1.0 & 1.0 & 1.0 & 1.0 & 1.0 & 1.0 & 1.0 & 0.0 & 0.0 & 0.0 \\
\hline 41 & 1.0 & 1.0 & 1.0 & 1.0 & 1.0 & 1.0 & 1.0 & 1.0 & 1.0 & 1.0 & 1.0 \\
\hline 42 & 0.0 & 0.0 & 0.0 & 0.0 & 0.0 & 0.0 & 0.0 & 0.0 & 1.0 & 1.0 & 1.0 \\
\hline 43 & 1.0 & 1.0 & 1.0 & 1.0 & 1.0 & 1.0 & 0.0 & 0.0 & 0.0 & 0.0 & 0.0 \\
\hline 44 & 0.0 & 0.0 & 0.0 & 0.0 & 0.0 & 0.0 & 0.0 & 0.0 & 0.0 & 0.0 & 0.0 \\
\hline 45 & 0.0 & 0.0 & 0.0 & 0.0 & 0.0 & 1.0 & 0.0 & 0.0 & 0.0 & 0.0 & 0.0 \\
\hline 46 & 0.0 & 0.0 & 0.0 & 0.0 & 0.0 & 0.0 & 0.0 & 0.0 & 0.0 & 0.0 & 0.0 \\
\hline 47 & 0.0 & 0.0 & 0.0 & 0.0 & 0.0 & 0.0 & 0.0 & 0.0 & 0.0 & 0.0 & 0.0 \\
\hline 48 & 0.0 & 0.0 & 0.0 & 0.0 & 0.0 & 0.0 & 0.0 & 0.0 & 0.0 & 0.0 & 0.0 \\
\hline 49 & 1.0 & 1.0 & 0.0 & 0.0 & 1.0 & 1.0 & 0.0 & 0.0 & 0.0 & 0.0 & 0.0 \\
\hline 50 & 1.0 & 1.0 & 0.0 & 0.0 & 1.0 & 0.0 & 0.0 & 0.0 & 0.0 & 0.0 & 0.0 \\
\hline Sum & 20.0 & 20.0 & 28.0 & 28.0 & 27.0 & 24.0 & 21.0 & 21.0 & 13.0 & 14.0 & 15.0 \\
\hline
\end{tabular}


Table 5: Stage 1 results for 22 versions in terms of fitting all packages and average fitness value (\%) for the runs where all packages fit. $\mathrm{T}=$ Tuning of the scoring weights (Yes/No); $\mathrm{C}=$ Components in the scoring function; $\mathrm{A}=$ Adjusted duplicate points used (Yes/No); Sum $=$ Sum of success rates of fitting all packages; Versions 12-22.

\begin{tabular}{|c|c|c|c|c|c|c|c|c|c|c|c|}
\hline Ver. & 12 & 13 & 14 & 15 & 16 & 17 & 18 & 19 & 20 & 21 & 22 \\
\hline $\mathrm{T}$ & $\mathrm{Y}$ & $\mathrm{Y}$ & $\mathrm{Y}$ & $\mathrm{Y}$ & $\mathrm{Y}$ & $\mathrm{Y}$ & $\mathrm{Y}$ & $\mathrm{Y}$ & $\mathrm{Y}$ & $\mathrm{Y}$ & $\mathrm{Y}$ \\
\hline $\mathrm{C}$ & $3 \mathrm{HA}$ & $3 \mathrm{HA}$ & $3 \mathrm{AH}$ & $3 \mathrm{AH}$ & $9+9$ & $9+9$ & 10 & 10 & 18 & 18 & 20 \\
\hline A & $\mathrm{N}$ & $\mathrm{Y}$ & $\mathrm{N}$ & $\mathrm{Y}$ & $\mathrm{N}$ & Y & $\mathrm{N}$ & $\mathrm{Y}$ & $\mathrm{N}$ & $\mathrm{Y}$ & $\mathrm{Y}$ \\
\hline 1 & 1.0 & 1.0 & 1.0 & 1.0 & 1.0 & 1.0 & 1.0 & 1.0 & 1.0 & 1.0 & 1.0 \\
\hline 2 & 1.0 & 1.0 & 1.0 & 1.0 & 1.0 & 1.0 & 1.0 & 1.0 & 1.0 & 1.0 & 1.0 \\
\hline 3 & 1.0 & 1.0 & 1.0 & 1.0 & 1.0 & 1.0 & 1.0 & 1.0 & 1.0 & 1.0 & 1.0 \\
\hline 4 & 1.0 & 1.0 & 1.0 & 1.0 & 1.0 & 1.0 & 1.0 & 1.0 & 1.0 & 1.0 & 1.0 \\
\hline 5 & 1.0 & 1.0 & 1.0 & 1.0 & 1.0 & 1.0 & 1.0 & 1.0 & 1.0 & 1.0 & 1.0 \\
\hline 6 & 0.0 & 0.0 & 0.0 & 0.0 & 0.7 & 1.0 & 1.0 & 1.0 & 1.0 & 1.0 & 1.0 \\
\hline 7 & 1.0 & 1.0 & 1.0 & 1.0 & 1.0 & 1.0 & 1.0 & 1.0 & 1.0 & 1.0 & 1.0 \\
\hline 8 & 0.0 & 0.0 & 0.1 & 0.1 & 0.9 & 1.0 & 1.0 & 1.0 & 1.0 & 1.0 & 1.0 \\
\hline 9 & 1.0 & 1.0 & 1.0 & 1.0 & 1.0 & 1.0 & 1.0 & 1.0 & 1.0 & 1.0 & 1.0 \\
\hline 10 & 1.0 & 1.0 & 1.0 & 1.0 & 1.0 & 1.0 & 1.0 & 1.0 & 1.0 & 1.0 & 1.0 \\
\hline 11 & 1.0 & 1.0 & 1.0 & 1.0 & 1.0 & 1.0 & 1.0 & 1.0 & 1.0 & 1.0 & 1.0 \\
\hline 12 & 0.0 & 0.0 & 0.0 & 0.0 & 1.0 & 1.0 & 0.6 & 0.7 & 1.0 & 1.0 & 1.0 \\
\hline 13 & 1.0 & 1.0 & 1.0 & 1.0 & 1.0 & 1.0 & 1.0 & 1.0 & 1.0 & 1.0 & 1.0 \\
\hline 14 & 0.0 & 0.0 & 1.0 & 1.0 & 1.0 & 1.0 & 1.0 & 1.0 & 1.0 & 1.0 & 1.0 \\
\hline 15 & 1.0 & 1.0 & 1.0 & 1.0 & 1.0 & 1.0 & 1.0 & 1.0 & 1.0 & 1.0 & 1.0 \\
\hline 16 & 0.0 & 0.0 & 0.0 & 0.0 & 1.0 & 1.0 & 1.0 & 1.0 & 0.7 & 0.9 & 1.0 \\
\hline 17 & 1.0 & 1.0 & 1.0 & 1.0 & 1.0 & 1.0 & 1.0 & 1.0 & 1.0 & 1.0 & 1.0 \\
\hline 18 & 1.0 & 1.0 & 0.0 & 0.0 & 0.0 & 0.6 & 1.0 & 1.0 & 1.0 & 1.0 & 1.0 \\
\hline 19 & 0.0 & 0.0 & 0.0 & 0.0 & 0.0 & 0.0 & 0.0 & 0.0 & 0.0 & 0.0 & 0.8 \\
\hline 20 & 0.0 & 0.0 & 1.0 & 1.0 & 1.0 & 1.0 & 1.0 & 1.0 & 1.0 & 1.0 & 1.0 \\
\hline 21 & 0.0 & 0.0 & 1.0 & 1.0 & 1.0 & 1.0 & 1.0 & 1.0 & 1.0 & 1.0 & 1.0 \\
\hline 22 & 1.0 & 1.0 & 1.0 & 1.0 & 1.0 & 1.0 & 1.0 & 1.0 & 1.0 & 1.0 & 1.0 \\
\hline 23 & 1.0 & 1.0 & 1.0 & 1.0 & 1.0 & 1.0 & 1.0 & 1.0 & 1.0 & 1.0 & 1.0 \\
\hline 24 & 0.0 & 0.0 & 1.0 & 1.0 & 1.0 & 1.0 & 1.0 & 1.0 & 1.0 & 1.0 & 1.0 \\
\hline 25 & 1.0 & 1.0 & 1.0 & 1.0 & 1.0 & 1.0 & 1.0 & 1.0 & 1.0 & 1.0 & 1.0 \\
\hline 26 & 0.0 & 0.0 & 1.0 & 1.0 & 1.0 & 1.0 & 1.0 & 1.0 & 1.0 & 1.0 & 1.0 \\
\hline 27 & 0.0 & 0.0 & 1.0 & 1.0 & 1.0 & 1.0 & 1.0 & 1.0 & 1.0 & 1.0 & 1.0 \\
\hline 28 & 1.0 & 1.0 & 1.0 & 1.0 & 1.0 & 1.0 & 1.0 & 1.0 & 1.0 & 1.0 & 1.0 \\
\hline 29 & 0.0 & 0.0 & 0.0 & 0.0 & 0.0 & 0.0 & 0.0 & 0.2 & 0.7 & 1.0 & 0.8 \\
\hline 30 & 1.0 & 1.0 & 0.0 & 0.0 & 1.0 & 1.0 & 1.0 & 1.0 & 1.0 & 1.0 & 1.0 \\
\hline 31 & 0.0 & 0.0 & 1.0 & 1.0 & 1.0 & 1.0 & 1.0 & 1.0 & 1.0 & 1.0 & 1.0 \\
\hline 32 & 0.0 & 0.0 & 1.0 & 1.0 & 1.0 & 1.0 & 1.0 & 1.0 & 1.0 & 1.0 & 1.0 \\
\hline 33 & 1.0 & 1.0 & 1.0 & 1.0 & 1.0 & 1.0 & 1.0 & 1.0 & 1.0 & 1.0 & 1.0 \\
\hline 34 & 0.0 & 0.0 & 1.0 & 1.0 & 1.0 & 1.0 & 1.0 & 1.0 & 1.0 & 1.0 & 1.0 \\
\hline 35 & 0.6 & 0.5 & 0.0 & 0.0 & 1.0 & 1.0 & 1.0 & 1.0 & 1.0 & 1.0 & 1.0 \\
\hline 36 & 1.0 & 1.0 & 1.0 & 1.0 & 1.0 & 1.0 & 1.0 & 1.0 & 1.0 & 1.0 & 1.0 \\
\hline 37 & 0.0 & 0.0 & 1.0 & 1.0 & 1.0 & 1.0 & 1.0 & 1.0 & 1.0 & 1.0 & 1.0 \\
\hline 38 & 0.0 & 0.0 & 0.0 & 1.0 & 1.0 & 1.0 & 1.0 & 1.0 & 1.0 & 1.0 & 1.0 \\
\hline 39 & 1.0 & 1.0 & 1.0 & 1.0 & 1.0 & 1.0 & 1.0 & 1.0 & 1.0 & 1.0 & 1.0 \\
\hline 40 & 1.0 & 1.0 & 1.0 & 1.0 & 1.0 & 1.0 & 1.0 & 1.0 & 1.0 & 1.0 & 1.0 \\
\hline 41 & 1.0 & 1.0 & 1.0 & 1.0 & 1.0 & 1.0 & 1.0 & 1.0 & 1.0 & 1.0 & 1.0 \\
\hline 42 & 0.0 & 0.0 & 1.0 & 1.0 & 1.0 & 1.0 & 1.0 & 1.0 & 1.0 & 1.0 & 1.0 \\
\hline 43 & 1.0 & 1.0 & 1.0 & 1.0 & 1.0 & 1.0 & 1.0 & 1.0 & 1.0 & 1.0 & 1.0 \\
\hline 44 & 1.0 & 0.0 & 1.0 & 1.0 & 1.0 & 1.0 & 1.0 & 1.0 & 1.0 & 1.0 & 1.0 \\
\hline 45 & 1.0 & 1.0 & 0.1 & 0.1 & 1.0 & 1.0 & 1.0 & 1.0 & 1.0 & 1.0 & 1.0 \\
\hline 46 & 0.0 & 0.0 & 1.0 & 1.0 & 1.0 & 1.0 & 1.0 & 1.0 & 1.0 & 1.0 & 1.0 \\
\hline 47 & 0.0 & 0.0 & 0.0 & 0.0 & 0.7 & 0.7 & 1.0 & 1.0 & 0.9 & 1.0 & 0.7 \\
\hline 48 & 1.0 & 1.0 & 0.0 & 0.0 & 0.0 & 0.3 & 0.0 & 0.1 & 1.0 & 1.0 & 1.0 \\
\hline 49 & 1.0 & 1.0 & 1.0 & 1.0 & 1.0 & 1.0 & 1.0 & 1.0 & 1.0 & 1.0 & 1.0 \\
\hline 50 & 1.0 & 1.0 & 0.0 & 0.0 & 1.0 & 1.0 & 0.9 & 0.9 & 1.0 & 1.0 & 1.0 \\
\hline Sum & 29.6 & 28.5 & 36.2 & 37.2 & 45.3 & 46.6 & 46.5 & 46.9 & 48.3 & 48.9 & 49.3 \\
\hline
\end{tabular}


Table 6: Stage 1 results for 22 versions in terms of fitting all packages and average fitness value (\%) for the runs where all packages fit. $\mathrm{T}=$ Tuning of the scoring weights (Yes/No); $\mathrm{C}=$ Components in the scoring function; $\mathrm{A}=$ Adjusted duplicate points used (Yes/No); Avg. = Average fitness values; Versions 1-11.

\begin{tabular}{|c|c|c|c|c|c|c|c|c|c|c|c|}
\hline Ver. & 1 & 2 & 3 & 4 & 5 & 6 & 7 & 8 & 9 & 10 & 11 \\
\hline $\mathrm{T}$ & $\mathrm{N}$ & $\mathrm{N}$ & $\mathrm{N}$ & $\mathrm{N}$ & $\mathrm{N}$ & $\mathrm{N}$ & $\mathrm{N}$ & $\mathrm{N}$ & $\mathrm{N}$ & $\mathrm{N}$ & $\mathrm{N}$ \\
\hline $\mathrm{C}$ & $3 \mathrm{HA}$ & $3 \mathrm{HA}$ & $3 \mathrm{AH}$ & $3 \mathrm{AH}$ & $9+9$ & $9+9$ & 10 & 10 & 18 & 18 & 20 \\
\hline A & $\mathrm{N}$ & Y & $\mathrm{N}$ & $\mathrm{Y}$ & $\mathrm{N}$ & $\mathrm{Y}$ & $\mathrm{N}$ & $\mathrm{Y}$ & $\mathrm{N}$ & $\mathrm{Y}$ & $\mathrm{Y}$ \\
\hline 1 & 98.5 & 98.5 & 98.6 & 98.6 & 97.8 & 97.8 & 98.7 & 98.6 & - & 98.5 & 97.4 \\
\hline 2 & - & - & 98.8 & 97.6 & 98.7 & 98.9 & 97.7 & 97.9 & 98.1 & 98.1 & 98.7 \\
\hline 3 & 99.5 & 99.5 & 99.5 & 99.5 & 99.5 & 99.5 & - & - & - & - & 99.7 \\
\hline 4 & 98.3 & 98.3 & 98.9 & 98.3 & 98.3 & 98.1 & 99.1 & 99.1 & 99.6 & 99.6 & 99.4 \\
\hline 5 & 99.5 & 99.5 & 99.3 & 99.3 & 99.2 & 99.2 & 98.8 & 98.8 & 99.2 & 99.3 & 99.4 \\
\hline 6 & - & - & - & - & - & - & - & - & - & - & - \\
\hline 7 & 98.3 & 98.3 & 99.2 & 99.1 & 99.2 & 99.3 & 97.8 & 98.2 & - & - & - \\
\hline 8 & - & - & - & - & - & - & - & - & - & - & - \\
\hline 9 & 98.4 & 98.7 & 99.0 & 99.0 & 98.9 & 98.5 & 98.1 & 98.2 & - & - & - \\
\hline 10 & 99.0 & 99.0 & 99.2 & 99.2 & 99.3 & 99.0 & 97.9 & 97.9 & - & - & 98.5 \\
\hline 11 & 98.5 & 98.5 & 98.5 & 98.4 & 97.9 & 97.5 & 98.3 & 98.2 & - & - & - \\
\hline 12 & - & - & - & - & - & - & - & - & - & - & - \\
\hline 13 & - & - & 98.9 & 98.8 & 99.0 & 98.6 & 98.2 & 98.2 & 99.1 & 99.1 & 99.0 \\
\hline 14 & - & - & 97.6 & 97.6 & - & - & - & - & - & - & - \\
\hline 15 & 97.5 & 97.6 & 96.9 & 97.0 & 96.9 & 96.8 & 97.6 & 97.6 & 99.4 & 99.4 & 99.4 \\
\hline 16 & - & - & - & - & - & - & - & - & - & - & - \\
\hline 17 & - & - & - & - & 99.4 & - & 99.5 & 99.5 & 99.5 & 99.5 & 99.8 \\
\hline 18 & 97.7 & 97.7 & - & - & - & - & 99.1 & 99.1 & - & - & - \\
\hline 19 & - & - & - & - & - & - & - & - & - & - & - \\
\hline 20 & - & - & - & - & - & - & - & - & - & - & - \\
\hline 21 & - & - & 99.1 & 99.1 & 98.2 & 99.0 & 98.6 & 98.6 & 99.1 & 99.1 & - \\
\hline 22 & 99.4 & 99.3 & 98.3 & 98.2 & 99.2 & 99.2 & 98.6 & 98.5 & 98.6 & 98.6 & 98.7 \\
\hline 23 & - & - & 99.6 & 99.6 & 98.8 & 99.5 & 98.4 & 98.4 & - & - & - \\
\hline 24 & - & - & - & - & - & - & - & - & - & - & - \\
\hline 25 & - & - & 98.5 & 98.5 & 98.3 & 98.2 & 98.6 & 99.8 & 98.6 & 98.7 & 98.6 \\
\hline 26 & - & - & 98.3 & 97.9 & 98.1 & - & - & - & 97.8 & - & - \\
\hline 27 & - & - & - & - & - & - & - & - & - & - & - \\
\hline 28 & 99.5 & 99.5 & 99.5 & 99.3 & 99.6 & 99.2 & 99.4 & 99.4 & - & - & - \\
\hline 29 & - & - & - & - & - & - & - & - & - & - & - \\
\hline 30 & - & - & - & - & - & - & - & - & - & - & - \\
\hline 31 & - & - & 97.6 & 97.6 & - & - & - & - & - & - & - \\
\hline 32 & - & - & 97.8 & 97.6 & 99.0 & - & - & - & - & - & - \\
\hline 33 & 99.0 & 99.0 & 98.6 & 98.6 & 98.5 & 99.5 & - & - & - & 98.7 & 98.8 \\
\hline 34 & - & - & 98.6 & 98.7 & - & - & - & - & - & - & - \\
\hline 35 & - & - & - & - & - & - & - & - & - & - & - \\
\hline 36 & 99.1 & 99.1 & 98.2 & 98.2 & 98.7 & 98.6 & 98.3 & 98.3 & - & - & - \\
\hline 37 & - & - & 99.1 & 98.9 & - & - & - & - & - & - & - \\
\hline 38 & - & - & - & - & - & - & - & - & - & - & - \\
\hline 39 & 98.1 & 98.1 & 97.9 & 97.9 & 97.7 & 97.7 & 98.1 & 98.1 & 98.4 & 98.3 & 98.1 \\
\hline 40 & 98.8 & 98.8 & 99.0 & 99.0 & 99.4 & 99.2 & 98.1 & 98.2 & - & - & - \\
\hline 41 & 99.5 & 99.4 & 99.5 & 98.5 & 99.2 & 99.2 & 99.4 & 99.1 & 99.4 & 99.4 & 99.4 \\
\hline 42 & - & - & - & - & - & - & - & - & 98.5 & 98.5 & 98.8 \\
\hline 43 & 97.7 & 97.6 & 97.4 & 97.4 & 97.6 & 97.6 & - & - & - & - & - \\
\hline 44 & - & - & - & - & - & - & - & - & - & - & - \\
\hline 45 & - & - & - & - & - & 98.7 & - & - & - & - & - \\
\hline 46 & - & - & - & - & - & - & - & - & - & - & - \\
\hline 47 & - & - & - & - & - & - & - & - & - & - & - \\
\hline 48 & - & - & - & - & - & - & - & - & - & - & - \\
\hline 49 & 99.6 & 99.6 & - & - & 99.8 & 99.8 & - & - & - & - & - \\
\hline 50 & 99.6 & 99.6 & - & - & 99.7 & - & - & - & - & - & - \\
\hline Avg. & 98.8 & 98.8 & 98.6 & 98.5 & 98.7 & 98.7 & 98.5 & 98.6 & 98.9 & 98.9 & 98.9 \\
\hline
\end{tabular}


Table 7: Stage 1 results for 22 versions in terms of fitting all packages and average fitness value (\%) for the runs where all packages fit. $\mathrm{T}=$ Tuning of the scoring weights (Yes/No); $\mathrm{C}=$ Components in the scoring function; $\mathrm{A}=$ Adjusted duplicate points used (Yes/No); Avg. = Average fitness values; Versions 12-22.

\begin{tabular}{|c|c|c|c|c|c|c|c|c|c|c|c|}
\hline Ver. & 12 & 13 & 14 & 15 & 16 & 17 & 18 & 19 & 20 & 21 & 22 \\
\hline $\mathrm{T}$ & $\mathrm{N}$ & $\mathrm{N}$ & $\mathrm{N}$ & $\mathrm{N}$ & $\mathrm{N}$ & $\mathrm{N}$ & $\mathrm{N}$ & $\mathrm{N}$ & $\mathrm{N}$ & $\mathrm{N}$ & $\mathrm{N}$ \\
\hline $\mathrm{C}$ & $3 \mathrm{HA}$ & $3 \mathrm{HA}$ & $3 \mathrm{AH}$ & $3 \mathrm{AH}$ & $9+9$ & $9+9$ & 10 & 10 & 18 & 18 & 20 \\
\hline A & $\mathrm{N}$ & $\mathrm{Y}$ & $\mathrm{N}$ & $\mathrm{Y}$ & $\mathrm{N}$ & $\mathrm{Y}$ & $\mathrm{N}$ & $\mathrm{Y}$ & $\mathrm{N}$ & $\mathrm{Y}$ & $\mathrm{Y}$ \\
\hline 1 & 98.6 & 98.5 & 98.7 & 98.7 & 99.3 & 99.3 & 99.7 & 99.7 & 99.7 & 99.7 & 99.5 \\
\hline 2 & 98.1 & 98.0 & 99.1 & 99.2 & 99.3 & 99.3 & 99.8 & 99.8 & 99.8 & 99.7 & 99.7 \\
\hline 3 & 99.5 & 99.5 & 99.5 & 99.5 & 99.7 & 99.7 & 99.8 & 99.7 & 99.8 & 99.8 & 99.8 \\
\hline 4 & 99.4 & 99.4 & 99.4 & 98.6 & 99.7 & 99.7 & 99.7 & 99.7 & 99.8 & 99.8 & 99.8 \\
\hline 5 & 99.6 & 99.6 & 99.3 & 99.3 & 99.7 & 99.8 & 99.8 & 99.8 & 99.9 & 99.9 & 99.8 \\
\hline 6 & - & - & - & - & 99.3 & 99.2 & 99.4 & 99.3 & 99.4 & 99.5 & 99.5 \\
\hline 7 & 99.2 & 99.2 & 99.4 & 99.3 & 99.6 & 99.6 & 99.5 & 99.6 & 99.7 & 99.7 & 99.5 \\
\hline 8 & - & - & 99.2 & 99.2 & 99.4 & 99.4 & 99.6 & 99.6 & 99.6 & 99.6 & 99.7 \\
\hline 9 & 99.2 & 99.2 & 99.0 & 99.0 & 99.5 & 99.5 & 99.6 & 99.7 & 99.7 & 99.8 & 99.7 \\
\hline 10 & 99.3 & 99.3 & 99.3 & 99.3 & 99.6 & 99.6 & 99.8 & 99.8 & 99.9 & 99.9 & 99.8 \\
\hline 11 & 98.8 & 98.6 & 98.8 & 98.5 & 99.2 & 99.1 & 99.7 & 99.7 & 99.8 & 99.7 & 99.7 \\
\hline 12 & - & - & - & - & 98.3 & 98.3 & 98.1 & 98.4 & 99.4 & 99.4 & 99.1 \\
\hline 13 & 98.7 & 98.7 & 98.9 & 98.9 & 99.5 & 99.4 & 99.6 & 99.6 & 99.5 & 99.5 & 99.2 \\
\hline 14 & - & - & 97.9 & 97.9 & 99.4 & 99.3 & 99.5 & 99.5 & 99.6 & 99.6 & 99.5 \\
\hline 15 & 98.5 & 98.3 & 97.8 & 97.8 & 99.3 & 99.3 & 99.7 & 99.7 & 99.8 & 99.7 & 99.6 \\
\hline 16 & - & - & - & - & 99.4 & 99.4 & 99.5 & 99.5 & 99.3 & 99.4 & 99.0 \\
\hline 17 & 99.4 & 99.4 & 99.6 & 99.5 & 99.7 & 99.7 & 99.8 & 99.8 & 99.9 & 99.9 & 99.9 \\
\hline 18 & 97.7 & 97.7 & - & - & - & 99.0 & 99.1 & 99.1 & 99.1 & 99.2 & 99.0 \\
\hline 19 & - & - & - & - & - & - & - & - & - & - & 99.6 \\
\hline 20 & - & - & 97.9 & 97.9 & 98.2 & 98.2 & 98.9 & 98.9 & 98.8 & 98.7 & 98.2 \\
\hline 21 & - & - & 99.3 & 99.3 & 99.6 & 99.6 & 99.8 & 99.8 & 99.8 & 99.7 & 99.7 \\
\hline 22 & 99.4 & 99.4 & 98.6 & 98.5 & 99.8 & 99.8 & 99.8 & 99.8 & 99.8 & 99.8 & 99.8 \\
\hline 23 & 99.5 & 99.5 & 99.6 & 99.7 & 99.8 & 99.8 & 99.8 & 99.8 & 99.8 & 99.8 & 99.8 \\
\hline 24 & - & - & 99.7 & 99.7 & 99.7 & 99.8 & 99.8 & 99.8 & 99.8 & 99.8 & 99.8 \\
\hline 25 & 99.4 & 99.4 & 99.3 & 99.3 & 99.6 & 99.6 & 99.8 & 99.9 & 99.9 & 99.9 & 99.9 \\
\hline 26 & - & - & 98.4 & 98.5 & 99.1 & 99.0 & 99.5 & 99.4 & 99.5 & 99.5 & 99.5 \\
\hline 27 & - & - & 98.1 & 98.0 & 98.8 & 98.7 & 99.1 & 99.1 & 99.5 & 99.5 & 99.3 \\
\hline 28 & 99.5 & 99.5 & 99.5 & 99.4 & 99.7 & 99.7 & 99.7 & 99.7 & 99.8 & 99.8 & 99.8 \\
\hline 29 & - & - & - & - & - & - & - & 99.4 & 99.6 & 99.7 & 99.7 \\
\hline 30 & 99.0 & 99.1 & - & - & 99.2 & 99.2 & 99.4 & 99.3 & 99.4 & 99.4 & 99.4 \\
\hline 31 & - & - & 97.7 & 97.7 & 99.2 & 99.2 & 99.2 & 99.2 & 98.6 & 98.9 & 98.8 \\
\hline 32 & - & - & 98.8 & 98.7 & 99.1 & 99.1 & 99.3 & 99.2 & 99.4 & 99.4 & 99.3 \\
\hline 33 & 99.5 & 99.5 & 99.6 & 99.6 & 99.8 & 99.8 & 99.8 & 99.8 & 99.9 & 99.9 & 99.9 \\
\hline 34 & - & - & 98.9 & 98.8 & 99.4 & 99.3 & 99.6 & 99.6 & 99.8 & 99.8 & 99.7 \\
\hline 35 & 99.7 & 99.7 & - & - & 99.8 & 99.8 & 99.9 & 99.9 & 99.9 & 99.9 & 99.8 \\
\hline 36 & 99.2 & 99.2 & 99.0 & 99.0 & 99.6 & 99.6 & 99.6 & 99.7 & 99.9 & 99.8 & 99.8 \\
\hline 37 & - & - & 99.3 & 99.3 & 99.5 & 99.5 & 99.7 & 99.7 & 99.8 & 99.8 & 99.7 \\
\hline 38 & - & - & - & 99.1 & 99.5 & 99.5 & 99.6 & 99.7 & 99.8 & 99.8 & 99.7 \\
\hline 39 & 98.2 & 98.1 & 98.0 & 98.0 & 99.4 & 99.4 & 99.7 & 99.7 & 99.9 & 99.9 & 99.8 \\
\hline 40 & 99.0 & 98.9 & 99.4 & 99.4 & 99.6 & 99.6 & 99.7 & 99.7 & 99.8 & 99.8 & 99.8 \\
\hline 41 & 99.5 & 99.5 & 99.5 & 99.2 & 99.8 & 99.8 & 99.7 & 99.7 & 99.9 & 99.9 & 99.9 \\
\hline 42 & - & - & 98.3 & 97.7 & 99.0 & 98.9 & 99.3 & 99.4 & 99.4 & 99.5 & 99.4 \\
\hline 43 & 98.2 & 98.2 & 99.2 & 99.1 & 99.3 & 99.3 & 99.6 & 99.6 & 99.7 & 99.7 & 99.7 \\
\hline 44 & 98.7 & - & 98.8 & 98.8 & 99.4 & 99.4 & 99.7 & 99.7 & 99.6 & 99.7 & 99.6 \\
\hline 45 & 98.7 & 98.8 & 97.7 & 97.6 & 99.4 & 99.4 & 99.6 & 99.6 & 99.4 & 99.4 & 99.3 \\
\hline 46 & - & - & 98.2 & 98.3 & 99.5 & 99.5 & 99.0 & 99.1 & 99.4 & 99.4 & 99.1 \\
\hline 47 & - & - & - & - & 98.9 & 98.6 & 98.8 & 99.1 & 98.9 & 99.1 & 98.4 \\
\hline 48 & 98.8 & 98.7 & - & - & - & 98.1 & - & 99.1 & 99.2 & 99.2 & 98.5 \\
\hline 49 & 99.7 & 99.7 & 99.6 & 99.6 & 99.8 & 99.8 & 99.9 & 99.9 & 99.9 & 99.9 & 99.9 \\
\hline 50 & 99.6 & 99.6 & - & - & 99.8 & 99.8 & 99.7 & 99.7 & 99.8 & 99.8 & 99.8 \\
\hline Avg. & 99.0 & 99.0 & 98.9 & 98.8 & 99.4 & 99.4 & 99.5 & 99.5 & 99.6 & 99.6 & 99.5 \\
\hline
\end{tabular}




\section{Comments on the Problem data}

In the Supplementary material data file Input50.cvs we give problem data for 1948 packages from 50 shipments, with 11 to 88 packages per shipment. Here follows a short description of all columns in the data file.

- Column 1 - Problem

Specifies which problem the package belongs to.

- Column 2 - Stage

Specifies which stage the package belongs to.

- Column 3 - Package

Unique package ID number.

- Column 4 - StandardSize

Specifies whether the package is of standard size (1) or not (0).

- Column 5 - Weight (kg)

Attribute 1 from Section 2.1 of the paper, package weight in kilograms.

- Column 6 - OuterLength (m)

Package outer length in meters.

- Column 7 - OuterWidth (m)

Package outer width in meters.

- Column 8 - OuterHeight (m)

Package outer height in meters.

- Column 9 - OuterVolume (cbm)

Package outer volume in cubic meters.

- Column 10 - HazardousContent

Attribute 5 from Section 2.1 of the paper, specifies whether the package contains hazardous content (1) or not (0).

- Column 11 - PalletAttached

Attribute 4 from Section 2.1 of the paper, specifies whether the package has pallets attached (1) or not (0). 


\section{- Column 12 - PalletOrientation}

Attribute 6 from Section 2.1 of the paper, where $0=$ runners parallell to length (standard), 1 = runners parallell to width, and $2=$ runners both directions.

\section{- Column 13 - OnlyOnFloor}

Attribute 7 from Section 2.1 of the paper, specifies whether the package must be placed on the floor (1) or not (0).

\section{- Column 14 - StackDifferentAbove}

Attribute 8 from Section 2.1 of the paper, specifies whether the package allows for a different package to be stacked above (1) or not (0).

\section{- Column 15 - StackSimilarAbove}

Attribute 9 from Section 2.1 of the paper, specifies whether the package allows for a similar package to be stacked above (1) or not (0).

\section{- Column 16 - StackingStrength}

Attribute 10 from Secton 2.1, indicates reduced or increased stacking strength.

- Column 17 - ECT (lb/in)

Part of attribute 2 from Section 2.1 of the paper, specifies the ECT factor for the package.

- Column 18 - Thickness (in)

Part of attribute 2 from Section 2.1 of the paper, specifies the thickness of the package's walls in inches.

\section{- Column 19 - WeightNoCorner (lb)}

Attribute 3 from Secton 2.1 of the paper, specifies the maximum load (in lbs) on top of the package if it provides support with only the lid. (Packages with a maximum load of $150 \mathrm{lbs}$ are crates of wood, all others are made of corrugated board.) 


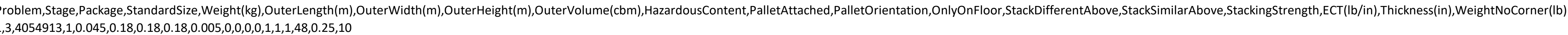
$1,3,4057805,1,2.415,0.33,0.33,0.033,0.0355,0,0,0,0,0,1,1,1,1,48,0.25,10$ $1,3,4059252,1,1.814,0.787,0.203,0.203,0.032,0,0,0,0,1,1,1,1,48,0.25,10$

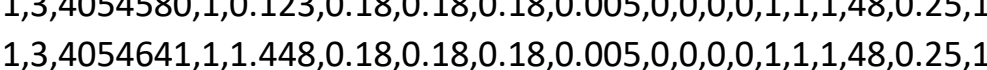
$1,3,4057849,1,1.49,0.28,0.28,0.28,0.021,0,0,0,0,0,1,1,1,48,0.25,10$ $1,3,4063662,1,6.12,0.635,0.635,0.432,0.174,0,0,0,0,0,1,1,48,0,0.25,10$ $1,1,4066715,0,171.7,1.778,0.584,1.143,1.186,0,0,1,1,0,1,1,1,1,80,0.025,150$

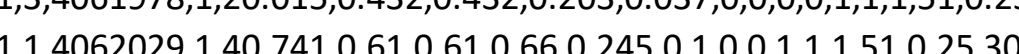
$1,1,4062069,1,71.57,0.584,0.381,0.559,0.124,0,1,1,0,1,1,1,51,02530$ $1,3,4063556,1,0.525,0.18,0.18,0.18,0.005,0,0,0,0,1,1,1,148,0.25,10$ $1,3,4064897,1,16.54,0.28,0.28,0.28,0.021,0,0,0,0,0,1,1,1,48,0.25,10$

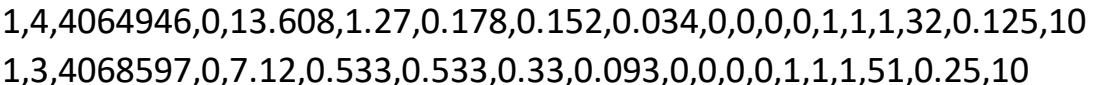
$1,1,4068714,1,354.01,0.61,0.61,0.66,0.245,0,1,0,0,1,1,1,51,0.025,30$ $1,3,40683399,1,20.108,0.5333,0.406,0.406,0.087,0,0,0,0,1,1,1,1,48,0.25,10$ $1,1,4068372,1,45,0.584,0.381,0.559,0.124,0,0,1,1,0,1,1,1,1,1,0.25,30$

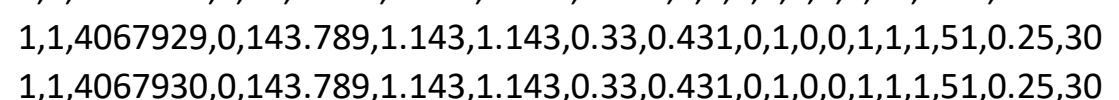
$1,3,4077039,121.475,0.787,0.203,0.203,0.032,0,0,0,0,1,1,1,48,0.25,10$ $1,4,4068525,0,18,1.143,0.762,0.152,0.132,0,0,0,0,0,1,1,1,11,0.25,10$ $1,3,4068542,1,16.54,0.432,0.432,0.203,0.037,0,0,0,0,0,1,1,1,51,0.25,10$

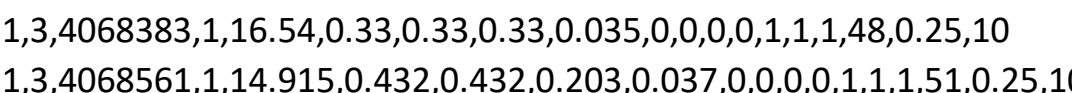
$1,1,4068519,0,034.338,0.584,0.3881,1.397,0.31,0,1,1,0,1,1,51,0.25,30$ $1,3,4073219,1,4,0.432,0.432,0.432,0.08,0,0,0,0,1,1,1,48,0.25,10$ $1,3,4073193,1,2.443,0.028,0.28,0.28,0.021,0,0,0,0,1,1,1,1,48,0.25,10$ $1,3,4073208,1,1,41,0.28,0.28028,0021,0,0,0,1,1,1,48,0.025,10$

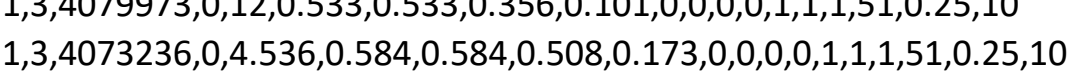
$1,3,4073240,0,18.337,0.533,0.406,0.305,0.066,0,0,0,0,0,1,1,1,51,0.25,10$ $1,1,4073159,1,49.94,0.584,0.381,0.559,0.124,0,1,1,0,1,1,1,51,0.25,30$

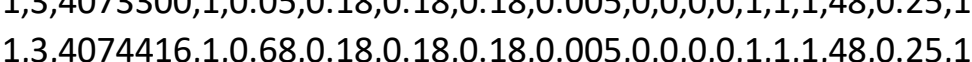

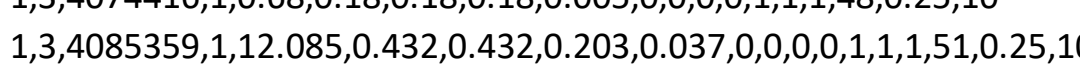
$1,3,4077032,1,0.477,0.28,0.28,0.28,0.021,0,0,0,0,1,1,1,48,0.25,10$

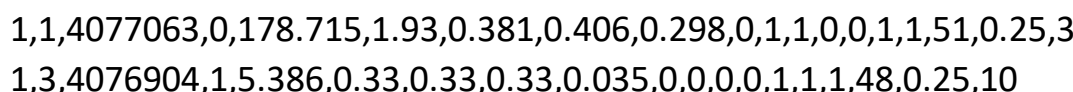
$1,3,4076904,1,5.386,0.033,0.33,0.33,0.035,0,0,0,0,0,1,1,1,4,48,0.25,10$ $1,1,4077158,1,60.418,0.5840 .0381,0.559,0.124,0,1,1,1,1,1,51,0.25$ $1,3,4076980,1,19.515,0.432,0.432,0.432,0.08,0,0,0,0,0,1,1,1,48,0.25,10$ $1,1,4077108,1,75.901,0.584,0.381,0.559,0.124,0,1,1,0,1,1,1,51,0.25,30$ $1,3,4077047,1,21.78,0.33,0.33,0.33,0.035,0,0,0,0,1,1,1,48,0.25,10$ $1,3,30080047,1,2.855,0.4322,0.432,0.0203,0.037,0,0,0,0,0,1,1,1,51,0.25,10$ $1,3,4081026,1,1,0.28,0.28,0.28,0.021,0,0,0,0,0,1,1,1,48,0.25,10$ $1,1,4083753,1,76.545,0.61,0.61,0.66,0.245,0,1,1,0,0,1,1,1,51,0.25,30$ $1,1,4083826,1,28.56,0.533,0.533,0.432,0.122,0,0,0,0,0,1,1,1,48,0.025,10$

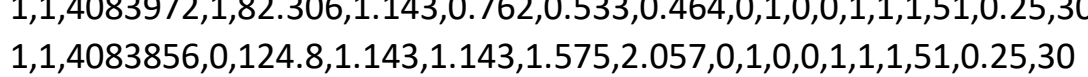
$1,1,4083942,1,31.88,1.143,0.762,0.533,0.0464,0,0,1,0,1,1,1,51,0.25,30$ $1,1,40838355,1,130.68,1.143,0.762,0.533,0.464,0,1,0,0,1,1,1,1,51,0.25,30$ 年,

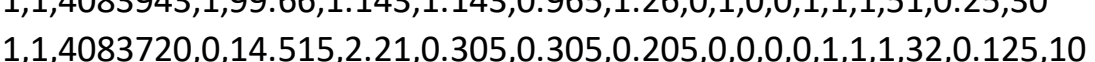
$1,1,4083977,0,178.715,1.956,0.406,0.356,0.0282,0,1,1,0,1,1,1,1,80,0.25,150$ $1,1,4084003,0,40.824,1.499,0.381,0.813,0.464,0,1,1,0,1,1,1,180,0.25,150$ $1,1,4083947,0,264.902,1.753,1.829,0.483,1.548,0,1,0,0,0,1,1,1,80,25$
$1,3,4085221,1,2.115,0.18,0.18,0.18,0.005,0,0,0,0,1,1,1,48,0.25,10$ $1,3,4086782,1,8.845,0.533,0.533,0.432,0.122,0,0,0,0,1,1,1,48,0.25,10$ $1,3,4087984,1,3.8,0.28,0.28,0.28,0.021,0,0,0,0,0,1,1,1,48,0.25,10$ $1,3,4087932,1,16.23,0.033,0.33,0.33,0.035,0,0,0,0,0,1,1,1,48,0.25,10$

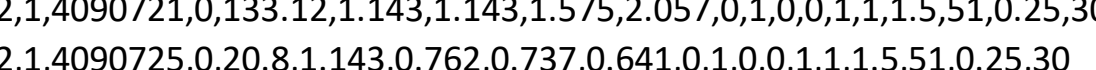
$2,1,4090660,0,199.32,1.143,1.143,1.245,1.626,0,1,0,0,0,1,1,1.5,5,51,0.25,30$ $2,1,4090686,0,174.405,1.143,1.143,1.321,1.725,0,1,0,0,1,1,1,1.5,51,0.25,30$ $2,1,4090689,1,99.66,1.143,1.143,0.965,1.26,0,1,0,0,0,1,1,1,51,0.25,30$ $2,1,4098412,14921,0.610 .01,0.6602450,010,0,1,1,151,0,25,30$ $2,3,4098320,0,7.96,0.432,0.0422,0.432,0.08,0,0,0,0,1,1,1,1.5,51,0.25,10$ $2,1,4098484,1,134,1.143,0.762,0.533,0.464,0,1,0,0,1,1,1,51,0.25,30$ $2,1,4098645,1,1,4,0.0 .584,0.381,0.559,0.124,0,0,1,1,0,1,1,1,51,0.025,30$ $2,1,4098554,1,1,130.451,1.143,0.762,0.533,0.464,0,1,0,0,0,1,1,1,51,0.025,30$ $2,1,4098483,1,196,1.143,0.762,0.533,0.464,0,1,0,0,1,1,1,51,0.2530$ $2,1,4098638,1,32.775,0.61,0.61,0.66,0.245,0,1,0,0,1,1,1,51,0.25,30$ $2,1,40986599,0,058.06,2.311,1.168,0.083,1.303,0,1,2,0,1,1,1,1.5,80,0,0.25,150$ $21,4098655,1,4994,058403810559,012401,101,1,510,2530$ $2,3,4098642,1,1,0.68,0.28,0.28,0.28,0.021,0,0,0,0,0,1,1,1,48,0.25,10$ $2,3,4098329,1,15.92,0.432,0.432,0.432,0.08,0,0,0,0,0,1,1,1,1,48,0.25,10$ $2,1,4098605,0,0199.32,1.143,1.1433,1.168,1.525,0,0,1,0,0,1,1,1,15,51,0.25,30$ $3,3,090748,1,13.060 .660 .66,0.06,0.287,0,0,0,0,1,1,4,1,0.25,10$ $3,1,4090778,1,30.572,1.143,0.7620 .914,0.796,0,1,0,0,1,1,1,80,0.25,30$ $3,3,4090805,1,0.3,0.18,0.18,0.18,0.005,0,0,0,0,1,1,1,48,0.25,10$ $3,1,4090596,1,49.83,1.143,0.762,0.0144,0.796,0,1,0,0,0,1,1,1,80,0.25,30$

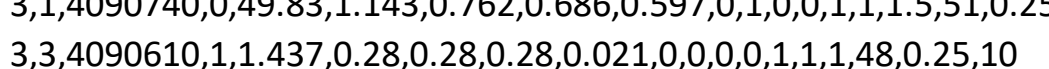
$3,3,4090724,1,0.675,0.432,0.432,0.203,0.037,0,0,0,01,1,1,51,0.25,10$ $3,1,4090589,1,49.83,1.143,0.762,0.914,0.796,0,1,0,0,01,1,1,80,0.25,30$ 3,1,4090585, $0,104.48,1.143,1,1.143,1,422,1.875,0,1,0,0,0,1,1,1,5,51,0.25,30$ $3,3,4090588,1,10.485,0.06,0.66,0.06,0.287,0,0,0,0,0,1,1,4,48,0.25,10$ $3,1,4090731,1,61.144,1.143,1.143,0.965,1.26,0,1,0,0,1,1,1,51,0.25,30$ $3,1,4090741,0,78.36,1.143,1.143,1.422,1.857,0,1,0,0,1,1,1.15,51,0.25,30$ $3,1,4090751,1,98.784,1.143,1.1433,1.575,2.2057,0,0,0,0,1,1,1,1,80,0.25,30$ 1. $31,4090777,0.23 .11 .143,0.762,0.635,0.553,0,1,0,0,1,1,1,51,0.2530$ $3,1,4090798,0,108.16,1.143,1.143,1.575,2.057,0,0,0,0,1,1,1,1.5,51,0.25,30$ $3,1,4090801,0,108.16,1.143,1.143,1.575,2.2057,0,1,0,0,0,1,1,1,1.5,51,0.25,30$ 年, $3,3,4090729,15.443,1016,0.28,0.28,0.079,0,0,0,0,1,1,51,0.25,10$ $3,3,4101111,1,0.35,0.18,0.18,0.18,0.005,0,0,0,0,0,1,1,1,48,0.25,10$ $3,3,4101164,1,2.745,0.28,0.28,0.28,0.021,0,0,0,0,0,1,1,1,1,48,0.25,10$ 年, $3,3,4101118,1,1.545,0.28,0.28,0.28,0.021,0,0,0,0,1,1,1,48,0.25,10$ $3,1,4101188,0,67.99,1.143,0.762,0.66,0.574,0,1,0,0,1,1,1.5,51,0.25,30$ $3,1,41012111,1,49.83,1.143,0.762,0.914,0.796,0,0,1,0,0,1,1,1,80,0.025,30$

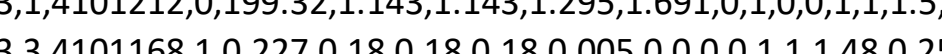
$3,4101113,1,0375,028,028,028,0021,0,0,0,1,1,1,48,025,10$ $3,3,4101177,0,24.915,0.61,0.61,0.584,0.217,0,0,0,0,0,1,1,1.5,51,0.25,10$ $4,1,4106592,0,595.512,1.143,1.143,1.397,1.825,0,1,0,0,1,1,1,1,51,0.25,30$

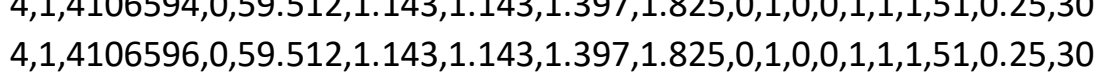
$4,1,4106539,0,59.512,1.143,1.143,1.3797,1.825,0,1,0,0,0,1,1,1,01,0.25,30$ $4,1,4106566,0,59.5121,1.143,1.143,1.397,1.1825,0,0,1,0,0,1,1,1,1,51,0.25,30$ $4,1,406567,12231,143,1,143,1397,1825,0,10,0,1,1,51,025,30$

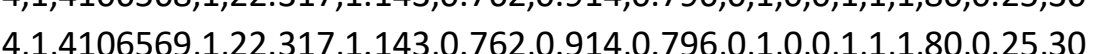
$4,1,4106531,1,29.756,1.143,1.143,0.065,1.26,0,1,0,0,0,1,1,1,51,0.025,30$ $4,1,4106536,0,59.512,1.143,1.143,1.397,1.825,0,0,1,0,0,1,1,1,151,0.25,30$

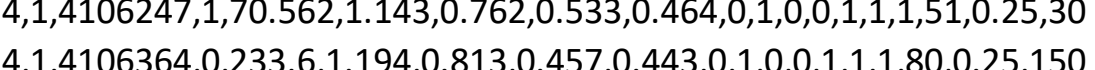
$41,4106589,1,52.07211430 .76209140 .7960,0,0,1,1,80,025,30$

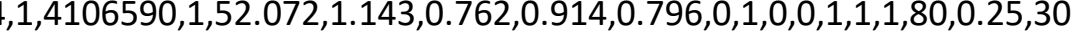
$4,1,41065991,1,52.072,1.1433,0.762,0.914,0.0796,0,1,0,0,0,1,1,1,180,0.25,30$ 4,1,4105908,0,0129.27, $1.1 .143,1.143,0.381,0.497,0,1,0,0,1,1,1,80,0.25,150$

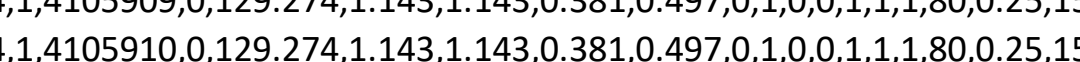
$4,1,4106445,0,19.68,1.143,1.143,1.397,1.825,0,1,0,0,0,1,1,1,51,0.25,30$ 
$4,3,4106446,1,3.28,0.635,0.635,0.432,0.174,0,0,0,0,0,1,1,1,48,0.25,10$ $4,1,4106460,0,0444.53,1.702,1.702,0.457,1.3233,0,1,0,0,1,1,1,3,51,0.25,30$ $4,1,4106261,0,0203.209,1.676,1.6,0.279,0.0748,0,0,1,0,0,1,1,1,3,80,0.25,150$ $4,1,4106349,0,249.48,2.083,2.057,0.279,1.195,0,1,0,0,0,1,1,3,300,0.25,150$ $4,1,4106245,1,56.735,1.143,0.762,0.533,0.464,0,1,0,0,1,1,1,51,0.25,30$ $4,1,4106375,0,226.94,1.219,0.864,0.914,0.962,0,0,1,0,0,1,1,1,1,80,0.25,150$ $4,1,4106586,0,59.512,1.143,1.143,1.397,1.825,0,0,0,0,1,1,1,1,51,0.25,30$

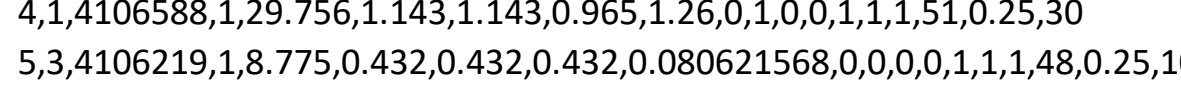
$5,1,4106482,0,252.024,2.286,1.143,2.032 .5,309408736,0,1,1,1,0,0,1,51,0.25,30$

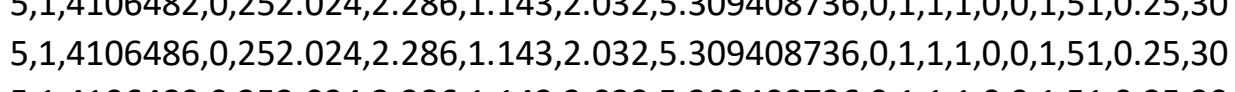
$5,1,4106489,0,252.024,2.286,1.143,2.032,5.3 .309408736,0,1,1,1,0,0,0,1,51,0.25,30$

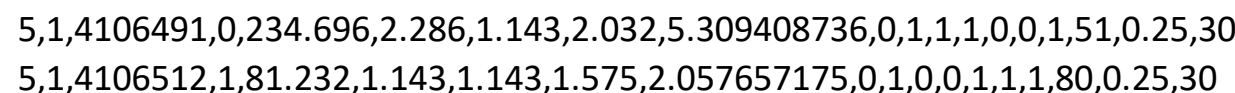
$5,1,4107649,0,240.119,2.286,1.143,1.93,5.04289314,0,1,1,0,0,1,51,0.25,30$ $5,1,4113047,0,92.986,1.143,1.143,0.381,0.497757069,0,0,1,0,0,1,1,1,1,80,0.25,150$ $5,1,4113585,0,01.75,1.143,0.762,0.0584,0.5086444144,0,0,1,0,0,1,1,1,1,51,0.25,30$ $5,1,14112909,0,193.5,2.794,0.889,0.0711,1.766028726,0,1,0,0,1,1,1,1,80,0.25,150$

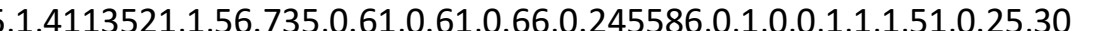
$5,1,4112942,0,78.471,1.346,0.0178,0.203,0.048636364,0,0,1,0,0,1,1,1,1,51,0.25,30$ $5,1,4113050,0,092.986,1.143,1.143,0.0381,0.497757069,0,1,0,0,1,1,1,1,80,0.25,150$ $5,1,4113051,0,92.986,1.143,1.143,0.381,0.497757069,0,1,0,0,0,1,1,1,80,0.25,150$ $5,1,4112923,0,099.79,1.143,1.1433,0.33,0.43112817,0,1,0,0,1,1,51,0.25,30$ $5,1,4113511,1,14.82,0.61,0.61,0.66,0.245586,0,1,0,0,1,1,1,51,0.025,30$ $5,1,4112925,0,099.79,1.143,1.143,0.33,0.43112817,0,1,0,0,1,1,1,51,0.25,30$

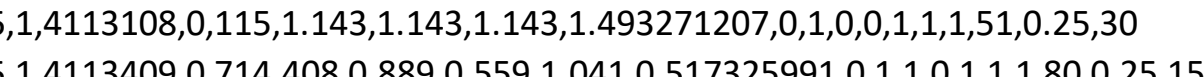

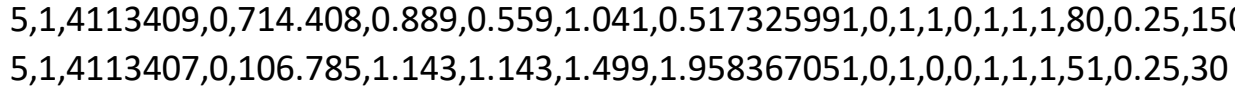
$5,1,4113588,1,51.66,1.143,0.762,0.533,0.464224878,0,1,0,0,0,1,1,1,1,0.25,30$ $5,1,4113390,1,143.34,1.143,0.762,0.533,0.4642248788,0,1,0,0,1,1,1,1,51,0.25,30$

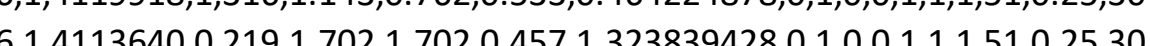

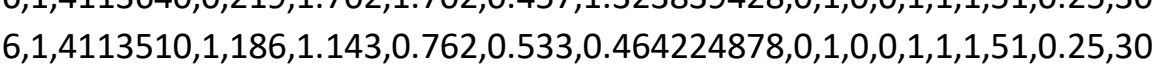
$6,1,4113518,1,260,1.143,0.762,0.533,0.464224878,0,1,0,0,1,1,1,51,0.25,30$ $6,1,4113524,0,186,1.143,0.762,0.584,0.5086444144,0,1,0,0,1,1,1,1,51,0.25,30$

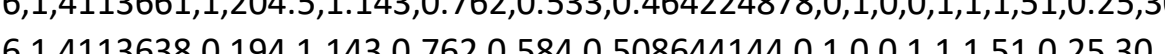

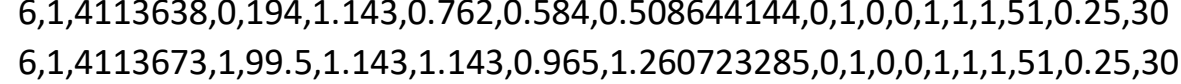
$6,1,4113652,1,102.5,0.61,0.61,0.66,0.245586,0,1,0,0,1,1,1,51,0.25,30$ $6,1,4120211,1,65,0.61,0.61,0.66,0.245586,0,1,0,0,1,1,1,1,51,0.25,30$

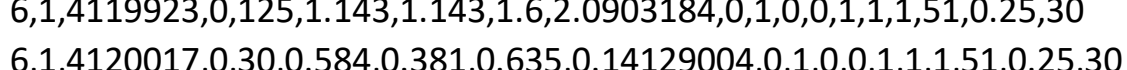
$6,1,4120193,0,115,1.143,0.762,0.94,0.81870804,0,0,1,0,0,1,1,1,1,1,1,25,30$ $6,1,4120194,0,121,1.143,0.762,0.94,0.81870804,0,1,0,0,1,1,1,51,0.25,30$ $6,1,4119582,0,180,1.143,1.143,0.0381,0.497757069,0,1,0,0,1,1,1,1,80,0.25,150$

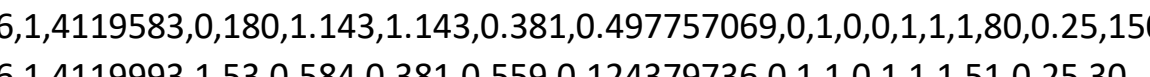

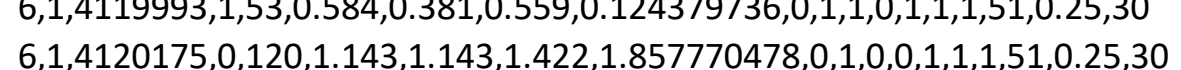
$6,1,4119924,0,0123,1.143,1.143,1.6,2.09003184,0,0,0,0,1,1,1,1,1,0,0.25,30$ $6,1,4119746,0,211,4.597,0.381,0.356,0.623518692,0,1,0,0,0,1,1,80,0.25,150$ $6,1,4119973,1,14,1.143,0.062,0.533,0.064224878,0,0,1,0,0,1,1,1,151,0.25,30$

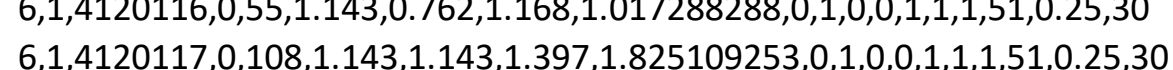
$6,1,4119954,0,77,1.143,0.762,1.016,0.884901456,0,1,0,0,1,1,1,51,0.25,30$ $6,1,4120150,1,105,1.143,0.762,0.533,0.464224878,0,1,0,0,1,1,1,51,0.25,30$ $6,1,4119581,0,103,1.143,1.143,0.33,0.043112817,0,1,0,0,1,1,1,51,0.25,30$

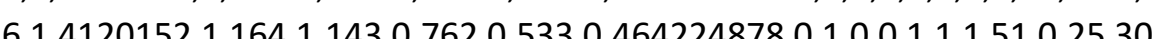
$6,1,4120153,1,50,0.584,0.381,0.559,0.124379736,0,1,1,0,0,1,1,1,51,0.25,30$ $6,1,4120195,0,109,1.143,0.762,0.94,0.81870804,0,0,1,0,0,1,1,1,51,0.25,30$ $6,1,4120196,0,199,1.143,0.762,0.944,0.81870804,0,1,0,0,0,1,1,1,1,11,0.25,30$ $6.1,41198390,180,1143,1.143,03810.497757069,0,0,0,1,1,1,0,025,150$ $6,1,4119840,0,180,1.143,1.143,0.33,0.43112817,0,1,0,0,1,1,1,80,0.25,150$ $6,1,4119841,0,180,1.143,1.143,0.381,0.497757069,0,1,0,0,1,1,1,80,0.25,150$ $6,1,4119842,0,180,1.143,1.143,0.381,0.497757069,0,1,0,0,0,1,1,1,80,0.25,150$ $6,1,41197544,0222980407110.08385841399672,0,1,0,0,1,1,1,11,0.25,30$ $7,1,4121374,0,197,1.19,0.81,0.46,0.44,0,1,2,0,1,1,1,80,0.25,150$ $7,1,4127059,0,00,1.14,1.14,0.033,0.43,0,1,0,0,1,1,1,151,0.25,30$ $7,1,4127308,1,99,1.14,1.14,0.97,1.26,0,0,1,0,0,1,1,1,51,0.025,30$ $7,1,4127296,1,17,1.14,0.076,0.91,0.08,0,1,0,0,0,1,1,1,1,80,0.25,30$ $7,1,4128461,0,218,2.79,0.89,0.46,1.14,0,1,1,1,1,1,1,80,0,25,150$ $7,1,4121184,0,337,1.78,0.76,0.38,0.52,0,1,1,0,1,1,1,1,80,0.25,150$ $7,1,4127298,1,76,1.14,0.76,0.91,0.8,0,0,1,0,0,1,1,1,80,0.25,30$ 7.,4128383, $2211,7,170461,320,01001,1,1,51,025,30$ $7,3,4127237,1,7.83,1.02,0.28,0.28,0.08,0,0,0,0,1,1,1,1,51,0.25,10$ $7,3,4127207,1,1.44,0.28,0.28,0.28,0.022,0,0,0,0,1,1,1,1,48,0.25,10$ $7,1,412727297,1,76,1,144,0.76,0.91,0.08,0,1,0,0,0,1,1,1,180,0.25,30$

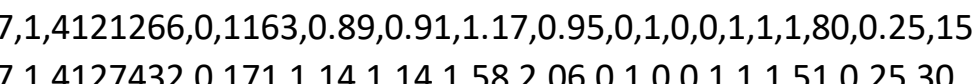
$7,1,4127355,0,294,2.29,1.14,1.75,4.58,0,1,0,0,0,1,1,1,51,0.25,30$ $7,1,41273994,0,0110,1.14,1.14,1,1.37,1.79,0,1,0,0,0,1,1,1,151,0.25,30$

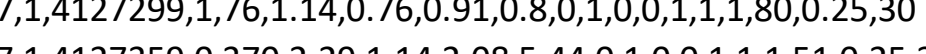

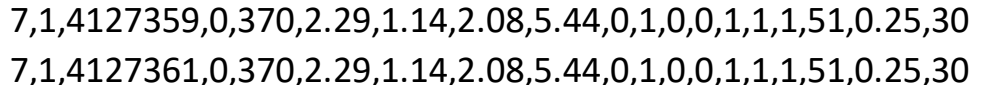
$7,1,4121256,0,336,1.14,0.76,0.56,0.49,0,0,0,0,1,1,1,51,0.25,30$ $7,1,4127396,0,53,1.14,0.76,1.12,0.97,0,1,0,0,1,1,1,151,0.25,30$ 年,

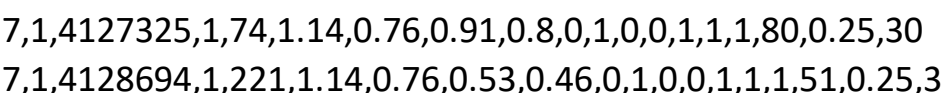
$7,1,4127323,1,74,1.14,0.76,0.91,0.8,0,1,0,0,1,1,1,10,0.25,30$ $8,1,4134807,1,162,1.143,0.762,0.914,0.796062924,0,0,1,0,0,1,1,1,80,0.025,30$ $8,1,4134832,1,161,1.143,0.762,0.914,0.796062924,0,1,0,0,1,1,1,1,80,0.25,30$ , $1,4134937,1,77,0.61,0.01,0,6,0245860,1,0,0,1,1,1,5,1,0.25,30$ $8,1,4135002,1,107,1.143,0.762,0.914,0.796062924,0,1,0,0,1,1,1,00.025,30$ $8,1,4135064,1,107,1.143,0.762,0.914,0.796062924,0,1,0,0,1,1,1,80,0.25,30$ $8,1,4135101,0,0219,1.702,1.702,0.457,1.3238394248,0,1,0,0,0,1,1,1,51,0.25,30$

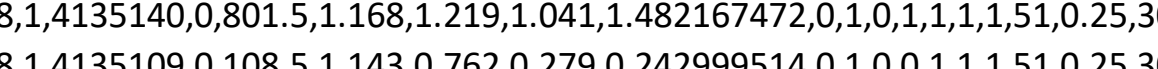
$81,4134448,0,124,1,143,1.143,033,0.43112817,0,1,0,0,1,1,1,51,0.25,30$ $8,1,4134449,0,124,1.143,1.143,0.33,0.43112817,0,1,0,0,1,1,1,51,0.025,30$ $8,3,4134878,0,0,7.75,0.559,0.0432,0.081,0.092006928,0,0,0,0,1,1,1,51,0.25,10$ $8,1,4135071,0,205,0.01,0.61,1.041,0.03873561,0,1,0,0,1,1,1,1,1,1,0.25,30$ $8,3,4134826,0,24.79,0.813,0.559,0.381,0.173151927,0,0,0,0,1,1,1,51,0.25,10$ $8,1,4135106,1,79,0.584,0.381,0.559,0.124379736,0,1,1,0,1,1,1,151,0.25,30$ $8,1,41349311,1,77,1.143,0.762,0.914,0.7960629244,0,1,0,0,1,1,1,1,80,0.25,300$ 8. $8,1,4134957,1,77,1.143,0.762,0.914,0.796062924,0,0,0,0,0,1,1,180,025,30$

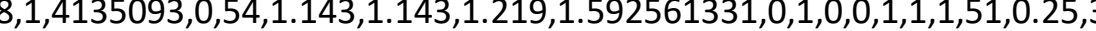
$8,1,4134997,0,69,1.143,0.0762,1.499,1.305578034,0,1,0,0,1,1,1,1,51,0.25,30$

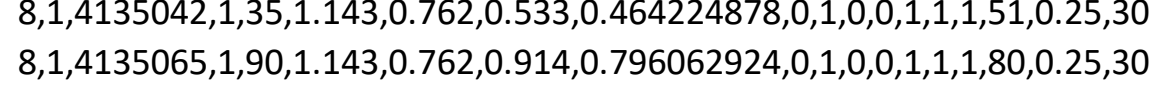
$8,1,4135103,0,275,1.702,1.702,0.457,1.323839428,0,1,0,0,1,1,1,51,0.25,30$ $8,1,4135092,0,337,1.168,1.143,0.508,0.678192192,0,1,0,0,1,1,1,2,80,0.25,150$ 年, $9,1,4142399,1,107,1.143,0.762,0.914,0.796,0,1,0,0,1,1,1,1,80,0.25,30$

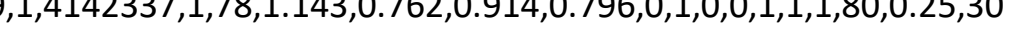
$9,1,4142427,0,162,1.016,1.016,0.254,0.262,0,1,2,0,1,1,2,51,0.25,30$ 9., $9,1,1442231,0,66,1.1430 .7620 .635,05530,0,0,0,1,1,51,025,30$ 9,1,4142345,1,78,1.143,0.762,0.914,0.796, $0,1,0,0,1,1,1,80,0.25,30$ $9,1,41423555,1,91,0.584,0.381,0.559,0.0124,0,1,1,0,1,1,1,1,51,0.25,30$ , $9,1,4139165,0,0103,1.143,0.0762,0.813,0.708,0,0,1,0,0,1,1,1,1,51,0.25,30$
$9,1,4142307,1,1087,1.143,0.0762,0.533,0.464,0,0,1,0,1,1,1,1,1,0.25,30$ 
$9,1,4142332,1,314,1.143,0.762,0.533,0.464,0,1,0,0,1,1,1,51,0.25,30$ $9,1,4141626,1,137,1.143,1.143,1.575,2.2058,0,1,0,0,0,1,1,1,1,80,0.25,30$

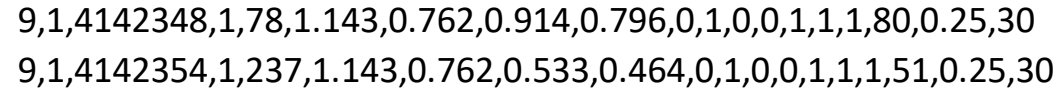
$9,1,4142309,1,193,1.143,0.0762,0.533,0.464,0,1,0,0,1,1,1,151,0.25,30$ $9,1,4142352,1,181,1.143,0.762,0.533,0.464,0,1,0,0,1,1,151,0.25,30$ $9,1,41422223,1,79,1.143,0.762,0.533,0.464,0,1,0,0,0,1,1,1,51,0.025,30$ $9,1,4136334,0,0223,2.794,0.014,0.0477,1.167,0,1,1,0,1,1,1,2,80,0.25,150$

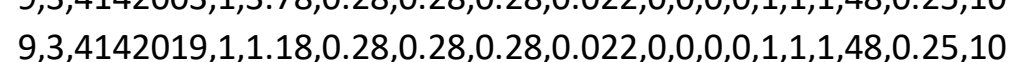
9.1.4135057,0,119,1.295, $0.66,0.406,0.347,0,0,2,2,1,1,2,00,0.25,150$ $9,1,4142137,1,149,1.143,0.762,0.965,0.84,0,1,0,0,1,1,1,1,51,0.25,30$ $9,1,4141696,0,190,1.143,1.143,0.33,0.431,0,1,0,0,1,1,1,51,0.25,30$

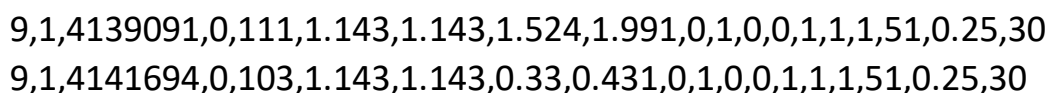
$9,1,4139088,0,106,1.143,1.143,1.524,1.991,0,1,0,0,1,1,1,51,0.25,30$ $9,1,4141692,0,100,1.143,1.143,0.33,0.431,0,1,0,0,1,1,1,151,0.25,30$ $9,1,4142313,0,100,1.143,1.1443,1.092,1.1427,0,1,0,0,1,1,1,1,51,0.25,30$

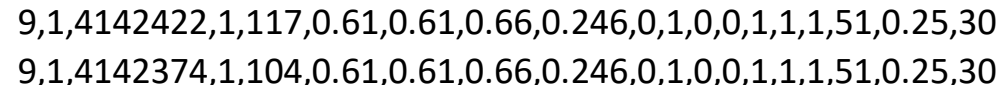
$9,1,4142373,1,104,0.61,0.61,0.66,0.246,0,1,0,0,1,1,51,0.25,30$ $9,1,4141624,1,67,1.143,0.762,0.965,0.84,0,1,0,0,1,1,1,1,1,0.025,30$ $9,1,4139106,0,0,40,1.143,0.762,0.787,0.685,0,0,0,0,0,1,1,1,51,0.025,30$ , 1,41414625,1,67,1.143,0.722,0.955,084,0,1,0,0,1,1,1,51,0.25,30 $9.1,4142228,0,111,1.143,1.143,1.397,1.825,0,1,0,0,1,1,151,0.25,30$ $10,1,4149013,0,124,1.143,1.143,0.33,0.431,0,1,0,0,1,1,1,1,51,0.025,30$

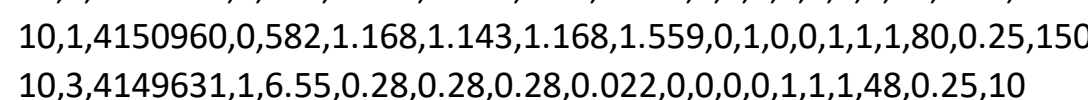

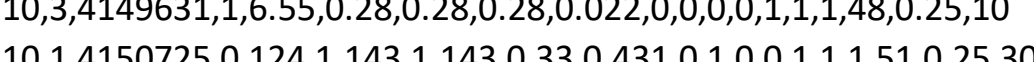
$10,1,41492988,0,100,1.143,1.1433,0.33,0.0431,0,1,0,0,0,1,1,1,51,0.025,30$ $10,1,4149673,0,40,1.143,1.143,0.279,0.364,0,1,0,0,1,1,1,51,0.25,30$ $10,1,4150728,0,124,1.143,1.143,0.033,0.431,0,1,0,0,0,1,1,1,51,0.25,30$ 年,

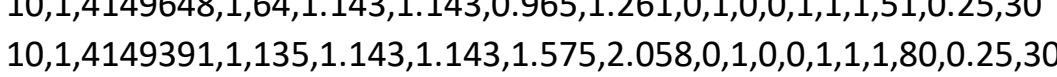
$10,1,4149513,0,367,2.438,1.143,2.108,5.874,0,1,1,0,1,1,1,51,0.25,30$ $10,1,41496100,0,367,2.438,1.143,2.2108,5.8744,0,1,1,0,1,1,1,1,51,0.25,30$ (1), $10,1,4149612,0,283,2.438,1.143,1.651,4.601,0,1,1,0,1,1,1,151,0.25,30$ $10,1,4149715,1,141,0.61,0.61,0.66,0.246,0,0,0,0,1,1,1,1,0,0.25,30-$ $10,1,4149710,1,103,0.61,0.61,0.66,0.246,0,1,0,0,1,1,1,1,01,0.25,30$ $10,1,4143480,0,449,1.72,1,702,0.66,1.912,0,1,0,0,1,1,1,1,51,0.25,30$ $10,1,4149707,1,103,0.061,0.61,0.66,0.246,0,0,0,0,0,1,1,1,51,0.25,30$ $10,1,4150920,1,246,1.143,0.762,0.533,0.464,0,0,1,0,1,1,1,1,51,0.25,30$ $10,1,4149685,1,205,1.143,0.762,0.533,0.464,0,1,0,0,0,1,1,1,51,0.25,30$ $10,1,445851,0,0290,0.94,0.71,0.0356,0.238,0,0,1,0,0,1,1,1,51,0.25,30$ $10,1,4150721,0,128,1.143,1.1433,0.33,0.431,0,1,0,0,0,1,1,1,1,0.0 .25,30$ $11,1,4578807,1,218,1.143,0.762,0.533,0.464,0,0,1,0,1,1,1,51,0.25,30$ $11,1,4578953,0,015,1,188,0.33,0.381,0.236,0,1,2,1,1,1,1,1,80,0.25,150$ $11,3,4579142,1,15.16,0.33,0.33,0.33,0.036,0,0,0,0,1,1,1,48,0.25,10$

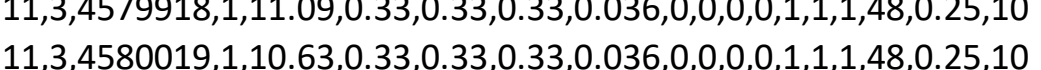
$11,3,4580115,1,9.78,0.28,0.28,0.28,0.022,0,0,0,0,0,1,1,1,48,0.25,10$ $11,3,4579125,1,7.68,0.28,0.28,0.28,0.022,0,0,0,0,1,1,1,4,48,0.25,10$ $11,3,4579168,1,1,676,0.028,0.28,0.28,0.022,0,0,0,0,1,1,1,1,48,0.25,10$

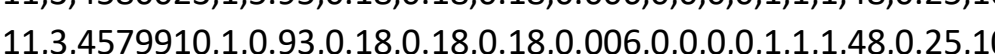
$11,3,4579161,1,0.27,0.18,0.18,0.18,0.006,0,0,0,0,1,1,1,48,0.25,10$ $11,1,4579082,1,53,0.584,0.381,0.559,0.1244,0,1,1,0,1,1,1,51,0.25,30$ $11,1,4579101,0,24,0.635,0.635,0.0813,0.328,0,1,1,0,0,1,1,0.5,80,0.25,150$ $11,4,4571013,1,1,0.89,0.28,0.28,0.28,0.022,1,0,0,0,0,1,1,1,48,0.25,10$ $11,1,4578971,0,164,1.854,0.991,0.457,0.84,0,1,2,1,1,1,1,80,0.25,150$ $11,4,4579087,0,13,1.1443,0.762,0.152,0.132,0,0,0,2,0,1,1,0,051,0.25,10$ $11,1,4580192,1,202,1.143,0.762,0.533,0.464,0,1,0,0,1,1,1,1,51,0.25,30$ $11,145789520,115,1.88033,03810.2360,121,1,1,1,0,51,0.25,30$ $11,1,4580000,1,122,1.143,0.762,0.533,0.464,0,1,0,0,1,1,1,51,0.25,30$ $11,1,4579923,1,82.6,61.143,0.762,0.533,0.464,0,1,0,0,1,1,1,51,0.25,30$ $11,1,4555067,0,065,6.02,0.0457,0.533,1,466,0,0,1,1,1,0,1,1,80,0.25,150$ $11,14582650,046,2845,0305,02030.1760,1,1,1,1,1,1,0,025,150$ $11,1,4582642,0,28,2.819,0.305,0.305,0.262,0,0,0,0,0,1,1,0.5,51,0.25,10$ $11,1,4579076,1,51,1.143,0.762,0.533,0.464,0,1,0,0,0,1,1,1,51,0.25,30$ $11,1,4579097,0,89,0.61,0.457,0.457,0.127,0,1,0,0,1,1,1,1,8,0.25,150$ $11,1,4582636,0,0295,1.829,0.61,0.483,0.539,0,0,1,2,1,1,1,1.5,580,0.025,150$ $11,3,4579091,0,5,5,0.635,0.61,0.33,0.128,0,0,0,0,0,1,1,1,0.5,51,0.25,10$ $11,1,4579093,1,56,0.61,0.61,0.66,0.246,0,1,0,0,1,1,1,51,0.25,30$ $11,1,4578970,0,119,1.93,0.66,0.737,0.939,0,0,1,2,1,1,1,1,80,0.25,150$ $11,1,4579081,1,060.5840,03105590,01240,0,1,1,1,1,1,15,02530$ $11,4,4579044,0,13,1.1433,0.762,0.152,0.132,0,0,0,0,0,1,1,0,51,0.25,10$ $11,3,4580035,1,25.69,1.016,0.28,0.28,0.08,0,0,0,0,1,1,1,1,51,0.25,10$ $11,4,4570819,0,1.04,0.406,0.33,0.203,0.027,1,0,0,0,1,1,0,0,51,0.25,10$ $11,4,4589427,0,3.02,0.0406,0.33,0.203,0.027,1,0,0,0,0,1,1,0,51,0.25,10$ $11,1,4579104,0,89,1.143,0.762,0.279,0.243,0,1,0,0,1,1,1,0,0.25,150$ $11,1,4579046,0,31,1.143,0.762,0.279,0.243,0,1,0,0,1,1,0,80,0.25,150$ $11,1,4578968,0,85,1.346,0.178,0.203,0.049,0,1,2,0,0,0,1,0,80,0.25,150$ $12,1,4154420,1,1,1.8,0.0 .584,0.381,0.559,0.124,0,1,1,0,1,1,1,51,0.25,30$ $12,1,4156090,186,0.584,0.381,0.559,0.124,0,1,0,1,1,151,025,30$ $12,1,4156054,1,65,0.584,0.381,0.559,0.124,0,1,1,0,0,1,1,1,11,0.25,30$

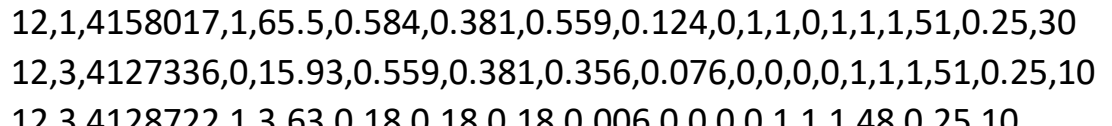
12.1.4139248,0.204,1.143,1.143,0.33,0.431,0,1,0,0,1,1,51,0.25,30 $12,1,4125898,0,0171,1.143,1.143,1.575,2.058,0,1,0,0,0,1,1,1,51,0.25,30$ $12,1,4139249,0,024,1.143,1.143,0.033,0.431,0,1,0,0,0,1,1,1,51,0.25,30$ 年,

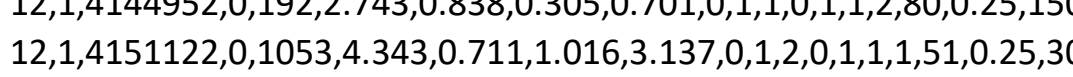

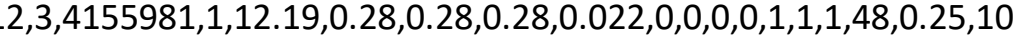
$12,1,4149639,0,115,1.676,0.813,1.321,1.8,0,1,1,2,1,1,0,1,80,0.25,150$ $12,1,4128780,0,312,3.323,0.035,1.067,2.048,0,1,2,0,0,1,1,1,51,0.25,30$ $12,3,4127373,1,9.01,0.4322,0.432,0.432,0.081,0,0,0,0,1,1,48,0.25,10$ $12,3,4149640,1,12.62,0.432,0.432,0.223,0.038,0,0,0,0,0,1,1,1,1,15,0,25,10$ $12,3,4122563,1,25.37,0.432,0.432,0.203,0.038,0,0,0,0,0,1,1,1,51,0.25,10$

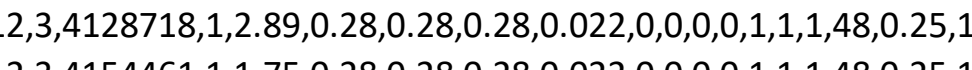
$12,3,4149716,1,23,028,028,028,0.022,0,0,0,1,1,1,48,025,10$ $12,3,4154333,1,1,76,0.33,0.33,0.33,0.036,0,0,0,0,0,1,1,1,48,0.25,100$ $12,3,4127348,1,0.46,0.18,0.18,0.18,0.006,0,0,0,0,1,1,1,48,0.025,10$

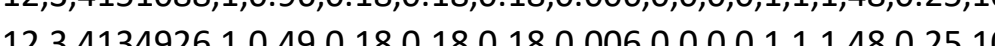
$12,1,4152363,3,0,1285,1.473,1,372,1,1.473,2.2977,0,0,1,1,1,1,28,0,025,150$ $12,1,4128598,1,34,0.61,0.61,0.66,0.246,0,1,0,0,1,1,1,151,0.25,30$ $12,1,4127460,1,111,1.143,0.0762,0.0333,0.064,0,0,1,0,0,1,1,1,1,51,0.25,30$

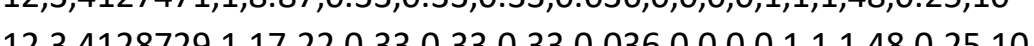

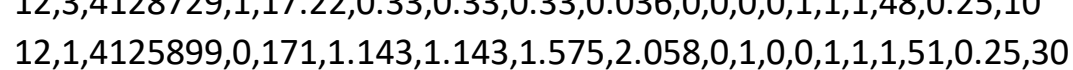
$13,1,41557996,0,180,1.1433,1.1434,0.381,0.498,0,1,0,0,0,1,1,1,1,10,0.25,150$
$13,1,4157101,0,180,1.143,1.143,0.381,0.498,0,1,1,0,0,1,1,1,80,0.25,150$ 13,1,457168,1,34,1.143,0.762,0.055,0.84,0,1,0,0,1,1,1,1,0.25,30 $13,1,4157110,1,80,1143,0.762,0.965,0.84,0,0,0,1,1,51,025,30$ $13,1,4157166,1,38,1.143,0.762,0.965,0.84,0,1,0,0,1,1,1,151,0.25,30$ $13,1,4157424,0,393,1.219,1.219,0.457,0.679,0,1,0,0,0,1,1,80,0.25,150$ $13,1,4157411,0,0390,1.219,1.219,0.457,0.679,0,1,0,0,1,1,1,1,80,0.25,150$

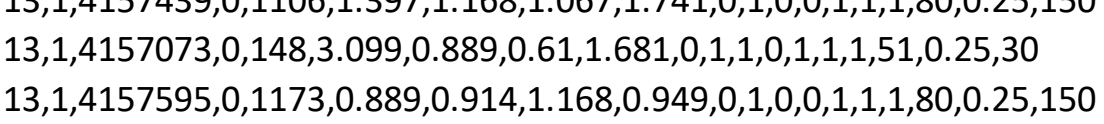


$13,1,4157061,0,1167,0.914,0.889,1.168,0.949,0,1,0,0,1,1,1,80,0.25,150$

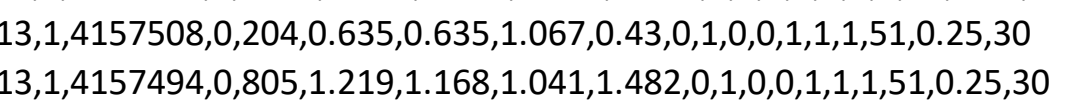
$14,1,4163749,0,01708,1.981,1.626,0.0533,1.717,0,1,1,0,1,1,1,2,51,0.25,30$ $14,1,4165389,1,82,1.143,1.143,0.965,1.261,0,0,0,0,0,1,1,1,51,0.25,30$
$14,1,4165490,1,34,1.143,0.762,0.533,0.464,0,1,0,0,1,1,151,25$
14,30 $4,1,4163747,0,1704,1.981,1.626,0.559,1.801,0,1,1,0,1,1,2,51,0.25,30$ $14,1,4165406,1,181,1.143,1.143,0.965,1.261,0,1,0,0,1,1,1,51,0.25,30$ $14,1,4165346,1,34,1.143,0.762,0.533,0.0464,0,1,0,0,0,1,1,1,51,0.25,30$

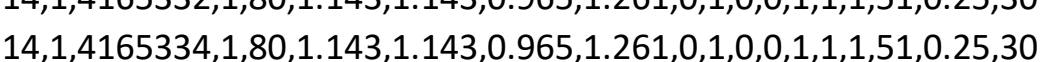
$4,1,4164980,0,180,1.143,1.143,0.381,0.498,0,1,0,0,1,1,2,80,0.25,150$ $14,1,4165333,1,80,1.143,1.143,0.965,1.261,0,1,0,0,1,1,1,51,0.25,30$ $14,1,4165338,1,79,1.143,1.143,0.965,1.261,0,1,0,0,0,1,1,1,51,0.25,30$

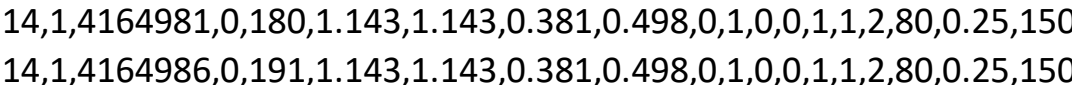
$14,1,4165339,1,182,1.143,0.762,0.533,0.464,0,1,0,0,1,1,1,51,0.25,30$ $14,1,4165396,1,48,1.143,0.762,0.914,0.796,0,1,0,0,1,1,1,18,0.25,30$ $14,3,4164972,1,1.64,0.28,0.28,0.28,0.022,0,0,0,0,0,1,1,1,48,0.025,10$

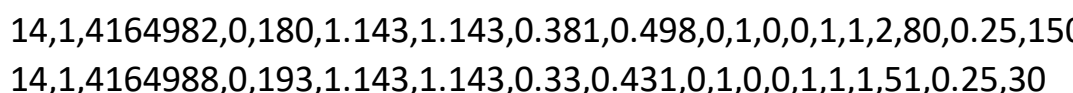
$14,1,4164989,0,193,1.143,1.143,0.33,0.431,0,1,0,0,1,1,1,51,0.25,30$ $14,1,4163830,0,01714,1.88,1.727,0.483,1.568,0,0,1,1,1,1,1,2,251,0.25,30$ $14,1,4165404,0,71,1.143,1.143,1.143,1.493,0,0,1,0,0,1,1,1,51,0.25,30$ $14,1,4163827,0,1,178,1.188,1.727,0.483,1.568,0,0,1,1,1,1,1,2,51,0.025,30$ $14,1,4165341,179,0.584,0.381,0.559,0.124,0,1,1,0,1,1,51,0.25,30$ $14,1,4165131,1,50,0.584,0.381,1.555,0.124,0,0,1,1,0,1,1,1,1,1,0.25,30$ $14,1,4165070,1,47,0.584,0.381,0.559,0.124,0,1,1,1,0,1,1,1,151,0.25,30$ (4,1,464887, $14,1,4165614,0,304,1.854,1.168,0.737,1.596,0,1,1,1,1,1,2,80,0.25,150$ $14,1,4165344,0,116,1.143,1.143,1.321,1.726,0,1,0,0,1,1,1,51,0.25,30$ $14,1,4165559,0,197,1.194,0.787,0.457,0.429,0,1,2,0,1,1,2,80,0.25,150$

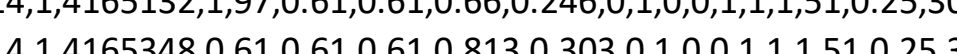

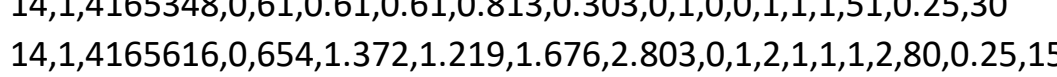
$15,1,4185656,1,38.2,0.584,0.381,0.559,0.124,0,1,1,0,1,1,1,51,0.25,30$ $15,1,4185564,1,115,0.061,0.61,0.66,0.246,0,1,0,0,0,1,1,1,51,0.25,30$

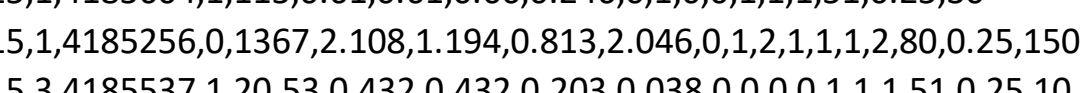
$15,3,4185568,1,13.58,0.432,0.432,0.203,0.038,0,0,0,0,0,1,1,1,1,0,025,10$.

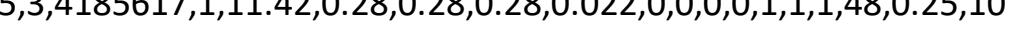
$15,3,4185500,1,4.03,0.28,0.28,0.028,0.022,0,0,0,0,0,1,1,1,48,0.25,10$ $15,3,4185579,0,22.61,0.584,0.084,0.0333,0.182,0,0,0,0,0,1,1,1,51,0.025,10$ $15,1,4173016,0,03.42,1.803,0.584,0.686,0.722,0,1,1,1,1,15,800,25,150$ $15,1,4169998,0,51.5,1.1433,0.762,0.279,0.243,0,1,0,0,1,1,1,80,0.25,150$ $15,1,14773018,1,71.67,1.1433,0.762,0.533,0.464,0,1,0,0,0,1,1,1,51,0.025,30$ $15,4,48983,0,17,1.143,0.762,0.152,0.132,0,0,0,0,0,0,0,0,11,0.25,10$ $15,1,4175899,1,34,0.584,0.381,0.559,0.0124,0,1,1,0,1,1,1,51,0.25,30$ $15,1,4181065,0,94,1.143,1.143,0.838,1.095,0,1,0,0,1,1,1,80,0.25,150$ $15,3,4177549,1,0.45,0.18,0.18,0.18,0.006,0,0,0,0,0,1,1,1,48,0.25,10$

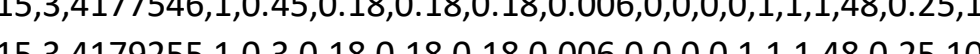
$15,1,1467925,0,0,345,1.702,1.092,0.0333,0.0991,0,1,0,0,0,1,1,2,280,0.25,150$ $15,1,4167928,0,345,1.702,1.092,0.533,0.991,0,1,0,0,1,1,2,80,0.25,150$ $15,1,14165776,1,55,1.143,0.762,0.914,0.0796,0,1,0,0,1,1,1,80,0.025,30$ $15,1,4165774,0,161,1.143,1.143,1,422,1.858,0,1,0,0,1,1,1,1,51,0.25,30$ $15,1,4175948,1,102,0.61,0.61,0.66,0.246,0,1,0,0,1,1,151,0.25,30$ $15,1,4165766,1,47.2,0.584,0.381,0.559,0.124,0,1,1,0,1,1,1,51,0.25,30$ $15,1,4169968,1,43.4,4.584,0.0381,0.559,0.124,0,0,1,0,1,1,1,151,0.25,30$ $15,4,4183529,0,0,1,0.406,0.33,0.03,0.027,1,0,0,0,0,0,0,0,0,11,0.25,10$ $15,14165786,1,134,1,143,1,143,0.955,1261,0,1,0,1,1,1,151,0.25,30$ $15,3,4169805,1,18.09,0.33,0.33,0.33,0.036,0,0,0,0,0,1,1,1,48,0.25,10$ (5,3,41839396,1,1.04,0.28, $0.28,0.028,0.022,0,0,0,0,0,0,1,1,1,48,0.25,10$ $15,3,4187207,1,12.04,0.033,0.33,0.33,0.036,0,0,0,0,0,1,1,1,48,0.25,10$ $15,3,4169965,1,18.21,0.432,0.432,0.432,0.081,0,0,0,0,0,1,1,1,48,0.25,10$
$15,3469762,12.66,0.432,0.432,0.203,0.038,0,0,0,0,1,1,51,0.25,10$ $15,3,4165535,1,8.31,0.28,0.28,0.28,0.022,0,0,0,0,0,1,1,48,0.25,10$ $15,3,3,4165634,1,3.44,0.28,0.028,0.28,0.022,0,0,0,0,0,1,1,1,148,0.025,10$ $15,3,4175972,1,7,42,2,0.28,0.028,0.28,0.0 .022,0,0,0,0,0,1,1,1,1,48,0.25,10$

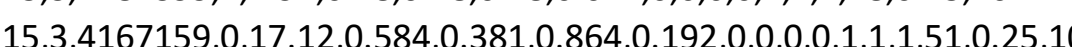

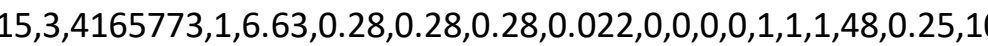
$15,3,4187263,1,1.65,0.28,0.28,0.28,0.022,0,0,0,0,1,1,1,4,48,0.25,10$ $15,3,4165530,1,6.36,0.028,0.28,0.28,0.022,0,0,0,0,1,1,1,1,48,0.25,10$ $16,1,4187196,0,142,1.753,0.356,0,033,0.206,0,0,1,1,0,1,1,1,80,0.25,150$ $16,1,4190253,0,14.39,1.524,0.178,0.178,0.048,0,0,0,0,0,0,0,0,51,0.25,10$ $16,1,4177283,0,24,0.584,0.584,0.33,0.113,0,1,0,0,1,1,1,80,0.025,150$
$16,1,1177715,1,116,0.61,0.61,0.66,0.246,0,1,0,0,1,1,1,51,0.25,30$

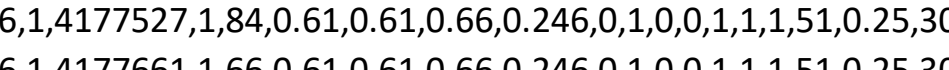
$16,1,4188575,0,9,1.626,0.457,0.178,0.132,0,0,0,0,0,0,0,0,51,0.25,10$ $16,1,4177536,0,122,0.61,0.61,0.635,0.236,0,1,0,0,1,1,1,80,0.25,150$ $16,1,4185655,1,1,01,0.584,0.381,0.559,0.124,0,1,1,0,1,1,1,51,0.25,30$ $16,1,4184084,1,57,0.584,0.381,0.559,0.124,0,1,1,0,1,1,1,1,151,0.25,30$ $16,1,4188577,0,9,1.626,0.457,0.178,0.1232,0,0,0,0,0,0,0,51,0.25,10$ $16,1,4177484,0,177,1.143,1.143,0.279,0.364,0,1,0,0,1,1,2,080,0.25,150$

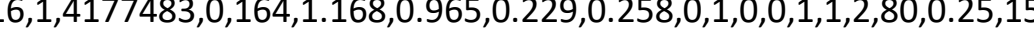

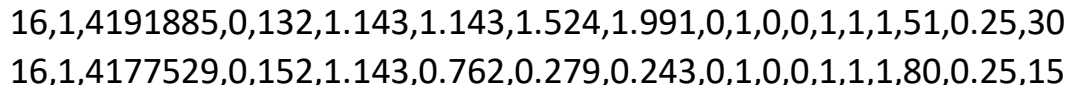
$16,1,4179408,0,39,1.143,1,1433,0.279,0.364,0,1,0,0,1,1,1,80,0.25,150$ $16,1,4177052,0,151,1.143,1.143,0.33,0.431,0,1,0,0,1,1,1,80,0.25,150$ $16,1,4177759,0,150,1.143,1.143,1.575,2.058,0,0,1,0,0,1,1,1,51,0.25,30$ (16,1,477784,1,97,143,1432, $5753,2058,0,0,0,1,1,51,0,25,30$ $16,1,4177285,1,197,1.143,0.762,0.533,0.464,0,1,0,0,1,1,151,0.25,30$ $16,1,4177733,0,118,1.143,1.143,1.321,1.726,0,1,0,0,1,1,1,51,0.25,30$ $16,1,41777118,0,107,1.016,0.279,0.046,0.0115,0,0,1,1,0,1,1,1,0.5,800,0.25,150$

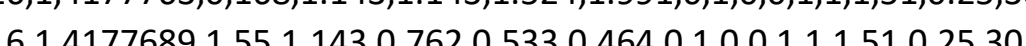
$16,1,4177473,1,43.6,1.143,0.762,0.533,0.0464,0,1,0,0,1,1,1,51,0.25,30$ $16,1,4184144,1,47,1.143,0.762,0.533,0.464,0,1,0,0,1,1,1,51,0.25,30$ $16,1,41777744,0,116,1.143,1.143,1.321,1.726,0,1,0,0,1,1,1,51,0.25,30$ 16,4,4777737,11,1.295, $1432,0.279,0.156,0,0,0,0,0,0,0,0,51,0.25,10$ $16,3,4177719,1,23.5,0.533,0.533,0.432,0.123,0,0,0,0,1,1,48,0.25,10$ $16,3,4167094,1,12.18,0.28,0.28,0.28,0.022,0,0,0,0,1,1,1,48,0.25,10$ $16,3,4191743,1,1.63,0.28,0.28,0.28,0.022,0,0,0,0,0,1,1,1,48,0.25,10$
$16,3,4182391,1,7.36,1.016,0.28,0.28,0.08,0,0,0,0,1,1,1,51,0.25,10$

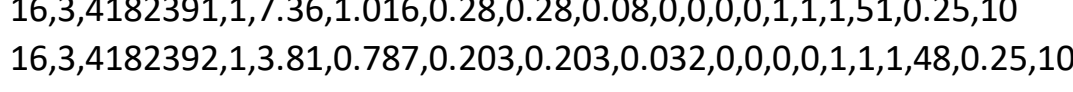
$16,3,4182396,1,1.81,0.787,0.203,0.2033,0.032,0,0,0,0,0,1,1,1,48,0.025,10$ $16,3,4165585,1,0.73,0.18,0.18,0.18,0.006,0,0,0,0,1,1,1,48,0.25,10$ $16,3,4163902,0,011.83,0.432,0.432,0.033,0.062,0,0,0,0,0,1,1,1,1,51,0.25,10$

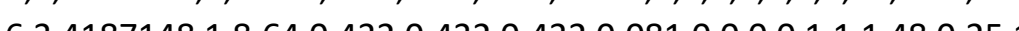
$16,3,31487148,1,1.64,0.0432,0.432,0.432,0.081,0,0,0,0,0,1,1,1,48,0.25,10$
$16,1,4177725,1,67,1.143,0.762,0.965,0.84,0,1,0,1,1,1,0.25,30$

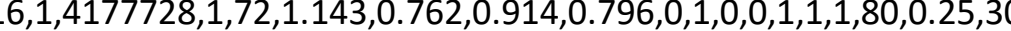
$16,1,4177695,0,089,1.143,0.762,1.372,1.195,0,1,0,0,0,1,1,1,51,0.25,30$ $16,1,4165783,0,231,1,143,1,143$ $16,1,4165783,0,231,1.143,1.143,1,295,1.692,0,0,1,0,0,1,1,1,51,0.025,30$
$17,1,4196183,1,110,1.143,0.7620 .9144,0.796,0,1,0,1,1,1,80,0.25,30$ $17,1,4196099,0,318,1.143,1.143,0.381,0.498,0,1,0,0,1,1,1,1,80,0.25,150$
$17,1,4196089,0,142,1.143,1.143,0.381,0.498,0,1,0,0,1,1,1,80,0.25,150$ $17,1,4196100,0,318,1.143,1.14,0.03$ $17,1,4196080,0,169,1.143,1.143,0.33,0.431,0,1,0,0,1,1,1,80,0.25,150$ $17,3,4196571,1,12,0.533,0.406,0.406,0.088,0,0,0,0,1,1,1,48,0.25,10$ $17,1,4196097,0,318,1.143,1.143,0.381,0.498,0,1,0,0,0,1,1,180,0.25,150$ $17,1,4196088,0,142,1.143,1.143,0.031,0.0488,0,1,0,0,0,1,1,1,80,0.25,150$ $17,1,4196102,0,318,1.143,1.143,0.381,0.498,0,0,1,0,0,1,1,1,1,80,0.25,150$ $17,1,4196083,0,169,1.143,1.143,0.33,0.431,0,1,0,0,1,1,1,1,80,0.25,150$ 
$17,1,4196104,0,0,180,1.143,1.143,0.381,0.498,0,1,0,0,1,1,1,180,0.25,150$ $17,1,4196107,0,0,180,1.143,1.143,0.3831,0.498,0,1,0,0,1,1,1,1,80,0.25,150$ $17,1,4196494,0,113,1.143,1.143,1.397,1.825,0,0,0,0,0,1,1,1,51,0.25,30$ $17,1,4196108,0,180,1.143,1.143,0.381,0.498,0,1,0,0,0,1,1,1,180,0.25,150$ $17,1,4196109,0,0180,1.143,1.143,0.381,0.498,0,1,0,0,1,1,1,00,0.25,150$ $17,1,4196570,0,112,1.143,1.143,1.397,1.825,0,1,0,0,1,1,1,51,0.25,30$ $17,1,4196110,0,180,1.143,1.143,0.381,0.498,0,1,0,0,0,1,1,1,80,0.25,150$ $17,14196035,0.117,1.143,143,1.168,1.5260,01,0,0,1,1,151,0.25,30$ $17,1,4196190,0,0258,1.575,0.813,0.711,0.91,0,1,0,0,1,1,1,180,0.25,150$ $18,1,4196670,0,1032,9.922,0.635,0.483,2.828,0,1,1,1,1,1,1,80,0.25,150$ $18,1,4196167,0,1039,9.246,0.635,0.483,2.836,0,1,1,1,1,1,0,1,80,0.25,150$ $18,1,4196508,0,668,1.194,0.813,0.533,0.517,0,1,1,2,1,1,1,2,280,0.25,150$ $18,1,4196696,0,1217,1.7271 .549,0.508,1.359,0,1,2,0,1,1,2,80,0.25,150$ $18,1,4196695,0,1202,1.727,1.549,0.508,1.359,0,1,2,0,1,1,2,2,80,0.25,150$ $18,1,4196692,0,098,1.219,1.168,1.041,1.482,0,0,1,0,1,1,1,2,80,0.25,150$

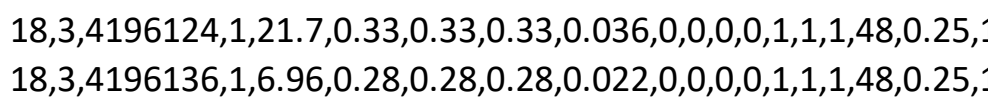

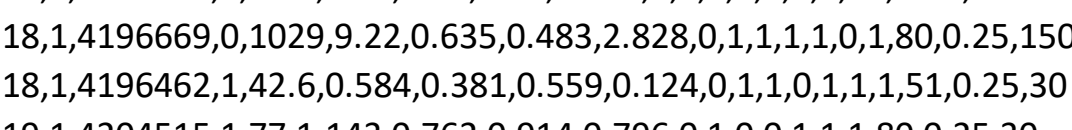
$19,1,4204515,1,77,1.143,0.762,0.914,0.796,0,1,0,0,0,1,1,1,80,0.25,30$ $19,1,4204069,0,107,0.61,0.61,0.035,0.236,0,1,0,0,0,1,1,1,80,0.25,150$ $19,1,4204513,1,75,1.143,0.762,0.914,0.796,0,1,0,0,1,1,1,00,0.25,30$ $19,1,4204017,0,100,1.143,1.143,0.33,0.431,0,1,0,0,1,1,1,80,0.25,150$ $19,1,4204018,0,100,1.143,1.143,0.33,0.431,0,1,0,0,1,1,1,180,0.25,150$ 年, $19,1,4204019,0,0,100,1.143,1.143,0.33,0.431,0,1,0,0,0,1,1,1,80,0.25,150$ $19,1,4204067,0,117,1.143,1.143,1.143,1.493,0,1,0,0,1,1,1,51,0.25,30$ $19,1,4204028,0,199,1.143,1.143,0.381,0.498,0,1,0,0,1,1,1,80,0.25,150$ $19,1,42040223,0,0191,1.143,1.143,0.381,0.498,0,0,1,0,0,1,1,1,180,0.25,150$ $19,1,4204033,0,142,1.143,1.143,0.381,0.498,0,1,0,0,1,1,1,80,0.25,150$ $19,1,42040622,0,100,1.143,1.143,1.422,1.858,0,1,0,0,0,1,1,1,51,0.25,30$

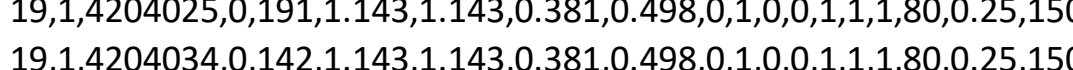
$19,1,4204143,0,0100,1.143,1.143,1.422,1.858,0,0,1,0,1,1,1,151,0.25,30$ $19,1,4204531,1,75,1.143,0.762,0.914,0.796,0,1,0,0,1,1,1,80,0.25,30$ $19,1,4204063,0,80,1.143,0.762,1.168,1.017,0,1,0,0,0,1,1,1,51,0.25,30$

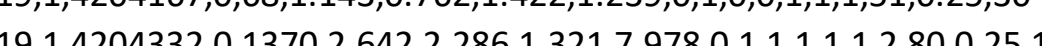

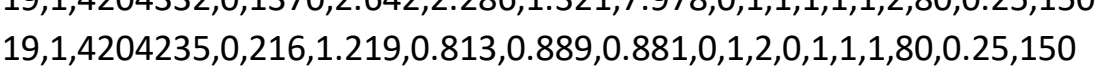
$20,1,4244709,0,40.5,1.143,1.143,0.305,0.398,0,0,1,0,0,1,1,1,80,0.25,150$ $20,1,4218263,0,235,1.143,1.168,1.295,1.729,0,1,0,0,0,1,1,1,80,0.25,150$ 年, $20,3,4198446,12330180180.180 .0060,0,0,0,1,1,1,0,25,10$ $20,3,4242808,1,1.54,0.18,0.18,0,18,0.006,0,0,0,0,1,1,1,48,0.25,10$ $20,3,4201768,1,1.19,0.18,0.18,0.18,0.006,0,0,0,0,0,1,1,1,48,0.025,10$ 年,

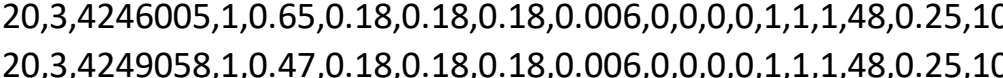
$20,3,4241145,1,0.43,0.18,0.18,0.18,0.006,0,0,0,0,1,1,1,1,48,0.25,10$ $20,3,42222876,1,24.63,0.432,0.432,0.432,0.0811,0,0,0,0,1,1,1,1,48,0.25,10$ $20,3,4240958,1,3.16,0.432,0.432,0.023,0.038,0,0,0,0,0,1,1,1,1,51,0.25,10$ $20,3,4217911,1,1760280228028022,0,0,0,0,1,1,1,4,4,0.25,10$

$20,3,4247647,1,1.36,0.28,0.28,0.28,0.022,0,0,0,0,1,1,1,48,0.25,10$ $20,3,4204774,1,1.22,0.28,0.28,0.28,0.022,0,0,0,0,0,1,1,1,48,0.25,10$ $20,1,4222812,1,1,132,1.143,0.762,0.533,0.464,0,1,0,0,0,1,1,1,51,0.025,30$

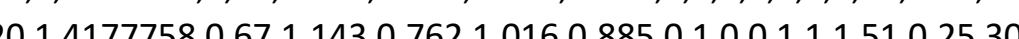
$20,1,4218265,1,98,1.143,1.143,0.965,1.261,0,1,0,0,1,1,1,151,0.25,30$ $20,1,41777422,0,95,1.143,1.143,1.067,1.394,0,1,0,0,0,1,1,1,51,0.25,30$ $20,1,4777732,0,123,1.143,1.143,1.422,1.858,0,0,1,0,0,1,1,1,1,51,0.25,30$

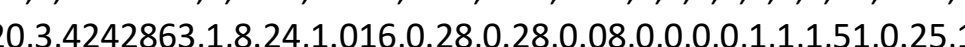
$20,3,4242873,1,8.24,1.016,0.28,0.28,0.08,0,0,0,0,0,1,1,1,51,0.25,10$ $20,3,4241076,1,2.47,0.432,0.432,0.203,0.038,0,0,0,0,0,1,1,1,51,0.25,10$ $20,3,4207911,1,2.26,0.033,0.033,0.033,0.036,0,0,0,0,0,1,1,1,148,0.25,10$ $20,32237851,1,10950333033033003600,0,1,1,1,025,250$ $20,3,4237853,1,6.21,0.33,0.33,0.33,0.036,0,0,0,0,1,1,1,48,0.25,10$ $20,3,4249157,1,6.65,0.33,0.33,0.33,0.036,0,0,0,0,0,1,1,1,48,0.25,10$ $20,3,4248923,1,3.08,0.033,0.33,0.033,0.036,0,0,0,0,0,1,1,1,48,0.25,10$ $20,14222410,0180,4.5720 .178,0.254,0.207,0,1,1,0,1,05,020,025,150$ $20,1,4222936,1,124,0.61,0.61,0.66,0.246,0,1,0,0,1,1,1,1,51,0.25,30$ $20,1,4222850,1,114.6,0.61,0.61,0.66,0.246,0,1,0,0,1,1,1,51,0.25,30$ $20,1,4222867,0,064.4,0.584,0.5844,0.737,0.0251,0,1,0,0,1,1,1,51,0.25,10$ $20,1,4204633,1,31,0.584,0.381,0.965,0.215,0,1,1,1,0,1,1,1,1,1,0.25,30$ $20,1,42222893,0,17.55,1.778,0.178,0.152,0.0488,0,0,2,0,0,0,1,0.5,532,0.125,10$ $20,1,4207459,0,0.95,1.6,0.2229,0.0229,0.084,0,0,0,0,0,1,1,1,1,51,0.25,10$ $20,1,4177671,1,50.8,0.584,0.381,0.559,0.124,0,0,1,1,0,1,1,1,51,0.025,30$ $20,1,4222856,1,210,1.143,0.762 .0533,0.464,0,1,0,0,1,1,51,02530$ $20,1,4222811,1,197,1.143,0.762,0.533,0.464,0,1,0,0,1,1,1,51,0.25,30$

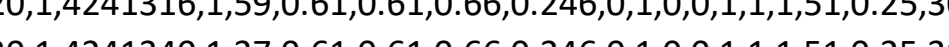

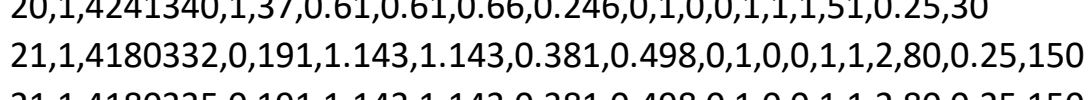
$21,1,4180335,0,191,1.143,1.143,0.381,0.498,0,1,0,0,1,1,2,80,0.25,150$ $21,1,4180474,1,65,1.143,0.762,0.965,0.84,0,1,0,0,1,1,1,51,0.25,30$ $21,1,4180334,0,191,1.143,1.143,0.381,0.498,0,1,0,0,0,1,1,2,80,0.25,150$

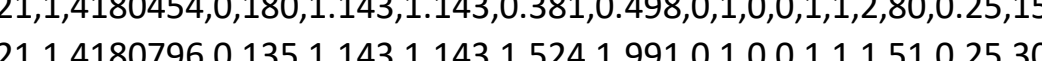
$21,1,4180455,0,180,1.143,1.143,0.381,0.498,0,1,0,0,1,1,2,280,0.25,150$ $21,1,4180449,0,193,1.143,1.143,0.33,0.431,0,1,0,0,1,1,1,51,0.25,30$ $21,1,4180482,0,132,1.143,1.1443,1.295,1.692,0,0,1,0,0,1,1,1,1,51,0.25,30$

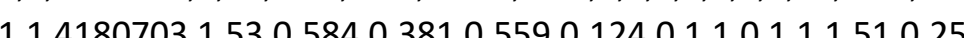
$21,1,4180526,1,1111,1.143,0.762,0.914,0.0796,0,0,1,0,0,1,1,1,80,0.25,30$

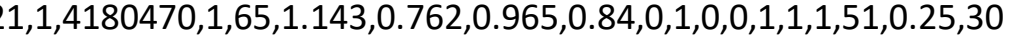
$21,1,4180927,0,52,0.61,0.61,0.889,0.331,0,1,0,0,0,1,1,1,51,0.25,30$

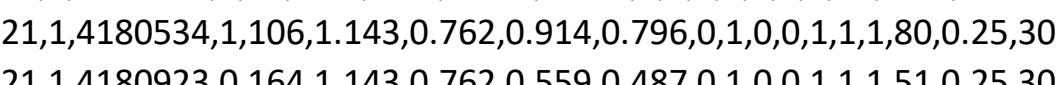
$21,1,4180406,0,152,2.438,0.914,0.254,0.566,0,1,2,0,1,1,2,80,0.25,150$ $21,1,4180456,0,180,1.143,1.143,0.381,0.498,0,1,0,0,1,1,2,80,0.25,150$ $21,1,4180441,0,0193,1.143,1.1443,0.33,0.431,0,1,0,0,0,1,1,1,51,0.25,30$ 年, $21,1,4180910,1,184,1.143,0.762,0.533,0.464,0,0,0,0,1,1,1,51,0.25,30$ $21,1,4180522,1,316,1.143,0.762,0.533,0.464,01,0,0,1,1,1,51,0.25,30$ $21,1,4180445,0,170,1.1433,1.143,0.33,0.431,0,1,0,0,1,1,1,51,0.25,30$

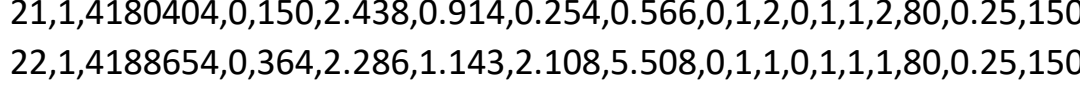
$22,1,4188655,0,0364,2.286,1.143,2.108,5.5080,0,1,1,0,1,1,1,80,0.25,150$ $22,1,41886660,0,364,2.286,1.143,2.2 .108,5.508,0,0,1,1,0,1,1,1,1,80,0.25,150$

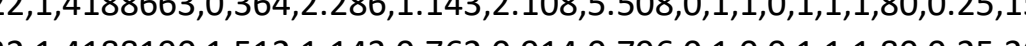

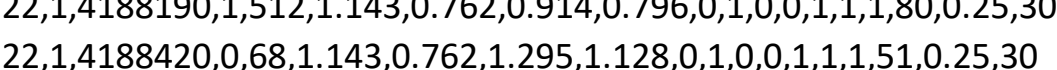
$22,1,4188425,1,104,1.143,0.762,0.914,0.796,0,1,0,0,1,1,80,0.25,30$ $22,1,4188430,1,106,1.143,0.762,0.914,0.796,0,1,0,0,1,1,1,80,0.25,30$

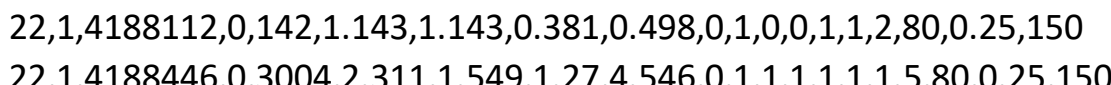
$22,1488167,170,0584,0381,0559,0.124,0,1,1,0,1,1,5,0,025,150$ $22,1,4188298,1,50,0.584,0.381,0.559,0.124,0,1,1,0,1,1,1,51,0.25,30$ $22,1,4188037,1,129,1.143,1.143,1.575,2.058,0,1,0,0,1,1,1,80,0.25,30$ $22,1,41884655,0,152,1,143,1,143,1.575,2.055,0,1,0,0,0,1,1,1,1,51,0.25,30$ $22,1,4188616,6,042,1.1443,0.762,0.686,0.0 .597,0,1,1,0,0,1,1,1,1,151,0.25,30$
$22,1,4188266,0,163,3.073,0.787,0.711,1.72,0,1,1,1,0,1,1,180,0.25,150$ 
$22,3,4188460,1,1.45,0.28,0.28,0.28,0.022,0,0,0,0,0,1,1,1,48,0.25,10$ $22,1,4188262,0,306,3.099,0.0762,0.737,1.74,0,1,1,1,0,1,1,1,80,0,0.25,150$

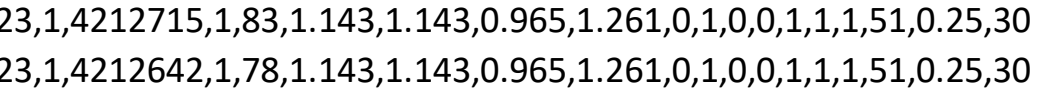
$23,1,42127799,1,78,1.143,1.143,0.965,1.261,0,1,0,0,0,1,1,1,51,0.25,30$ $23,1,4212644,1,77,1.143,1.143,0.965,1.261,0,1,0,0,0,1,1,151,0.25,30$ $23,1,4212695,1,5,1.143,1.143,0.965,1.261,0,1,0,0,1,1,1,51,0.25,30$ $23,1,4212942,1,168,1.143,0.762,0.5333,0.464,0,1,0,0,0,1,1,1,51,0.25,30$

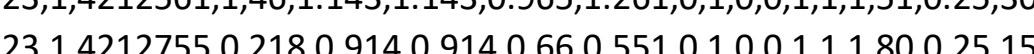
$23,1,4212818,0,222,1.016,1.016,0.66,0.681,0,1,0,0,1,1,1,80,0.25,150$ $23,1,4212732,1,87,0.61,0.61,0.66,0.246,0,1,0,0,0,1,1,1,51,0.25,30$ $23,1,4212805,1,179,0.61,0.061,0.66,0.246,0,1,0,0,1,1,1,51,0.25,30$ 23,1,4212377, 168,1143,0.762, $0.6550 .084,0,1,0,0,1,1,1,1,00,0.25,150$ $23,1,4212573,0,58,1.143,0.762,1.092,0.951,0,1,0,0,1,1,151,0.25,30$ $23,1,4212628,1,38,1.143,0.762,0.533,0.464,0,1,0,0,1,1,1,51,0.25,30$

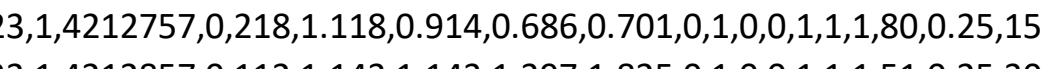
$23,1,4212857,0,112,1.143,1.143,1.397,1.825,0,1,0,0,1,1,1,1,51,0.25,30$

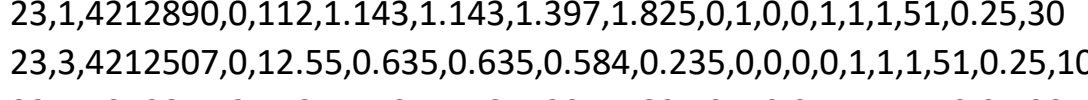
$23,1,42122841,0,110,1.143,1.143,1.397,1.825,0,1,0,0,0,1,1,1,51,0.25,30$ $23,1,4212565,0,100,1.143,1.143,1.397,1.825,0,0,1,0,0,1,1,1,1,51,0.25,30$ $23,1,4212572,0,99,1.143,1.143,1.397,1.825,0,1,0,0,1,1,1,51,0.25,30$ $23,1,4212567,0,91,1.143,1.143,1.397,1.825,0,1,0,0,1,1,1,151,0.25,30$ $23,1,4212482,0,318,1.143,1,143,0.381,0.498,0,0,1,0,0,1,1,1,80,0.25,150$

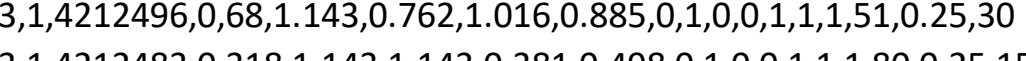
$23,1,4212938,0,081,1.143,1.1443,1.219,1.593,0,0,1,0,0,1,1,1,51,0.25,30$ $23,1,4212484,0,318,1.143,1.143,0.381,0.498,0,1,0,0,1,1,1,80,0.25,150$ $23,1,42122839,0,099,1.143,1.143,1.473,1.924,0,0,1,0,0,0,1,1,1,51,0.025,30$

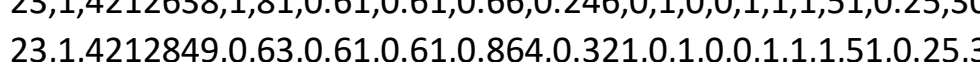

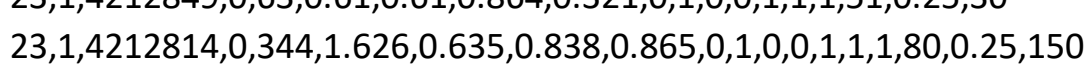
$23,1,4212634,0,303,1.067,1.067,0.635,0.723,0,1,0,0,1,1,1,80,0.25,150$ $24,1,4271447,1,77,1.143,0.762,0.914,0.796,0,1,0,0,1,1,1,80,0.25,30$ $24,1,4271162,0,191,1.43,1.143,0.381,0.498,0,0,0,0,0,1,1,1,80,0.25,150$ $24,1,4271446,177,1433,0.762,0.914,0.796,0,1,0,0,1,1,80,025,30$ $24,1,4271440,0,310,1.219,0.813,0.381,0.378,0,1,0,0,1,1,1,80,0.25,150$ $24,1,4271445,1,77,1.143,0.762,0.0914,0.796,0,1,0,0,1,1,1,80,0.25,30$ $24,1,427114,0,80,1.143,1.143,0.381,0.498,01,0,1,1,1,00,0.25,150$ $24,1,4271301,0,099,1.143,1.143,1,397,1.825,0,0,1,0,0,1,1,1,51,0,025,30$ $24,1,4271167,0,180,1.143,1,143,0.381,0.498,0,1,0,0,1,1,1,80,0.25,150$ $24,1,4271176,0,193,1.143,1.143,0.33,0.431,0,1,0,0,1,1,1,80,0.25,150$

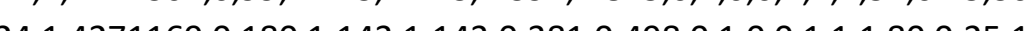
$24,1,4271169,0,180,1.143,1.143,0.381,0.498,0,1,0,0,1,1,1,1,80,0.25,150$
$24,1,4271304,0,133,1.143,1.143,0.279,0.364,0,1,0,0,1,1,1,80,025,150$ $24,1,4271356,0,99,1.143,1.143,1397,1.825,0,1,0,0,1,1,1,51,0.25,30$ $24,1,4271170,0,180,1.143,1.143,0.381,0.498,0,1,0,0,1,1,1,80,0.25,150$ $24,1,4271263,0,228,1.067,1.067,0.61,0.694,0,0,1,0,0,1,1,1,80,0.25,150$

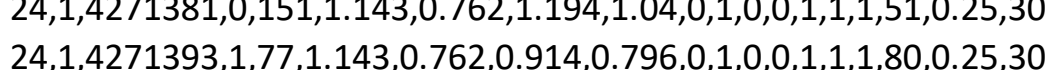
$24,1,4271443,177,1.143,0.762,0.914,0.796,0,1,0,0,1,1,80,0.25,30$ $24,1,4271442,1,77,1.143,0.762,0.914,0.796,0,1,0,0,1,1,1,180,0.25,30$

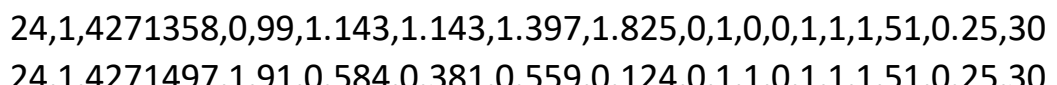
$24,1.471554,1,190,0.584,0.381,0.559,0.124,0,1,1,0,1,1,1,1,0.2530$ $24,1,4271449,1,429,1.143,0.762,0.914,0.796,0,1,0,0,1,1,1,80,0.25,30$ $24,1,4271379,1,48,1.143,0.762,0.914,0.796,0,1,0,0,1,1,1,80,0.25,30$ $24,1,4271389,0,220,1.1 .194,0.8388,0.914,0.915,0,0,0,0,1,1,1,1,80,0.25,150$

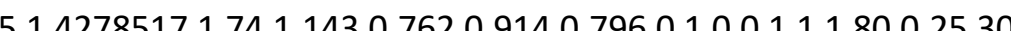
$25,1,4278611,1,266.5,1.143,0.762,0.533,0.464,0,1,0,0,0,1,1,1,1,1,0.25,30$ $25,1,4278518,1,14,1.143,0.762,0.914,0.796,0,1,0,0,1,1,1,80,0.25,30$ $25,1,42784433,0,139,1.143,1.1443,0.33,0.431,0,1,0,0,0,1,1,1,80,0.25,150$

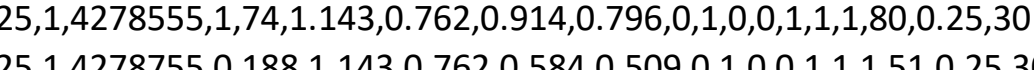
$25,1.4278445,0,139,1.143,1.143,0.33,0.431,0,1,0,0,1,1,1,80,0.25,150$ $25,1,4278203,0,67,1.143,0.0762,1.016,0.8855,0,1,0,0,1,1,1,51,0.25,30$ $25,1,4278765,1,34,1.143,0.7262,0.533,0.0464,0,0,1,0,0,1,1,1,51,0.025,30$

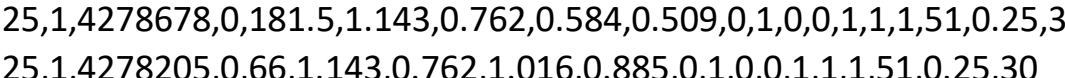
$25,1,4278446,0,139,1.143,1.143,0.33,0.431,0,1,0,0,1,1,1,80,0.25,150$ $25,1,4278685,0,114,1.143,1.143,1.397,1.825,0,1,0,0,1,1,1,51,0.025,30$ $25,1,42783929,0,100,1.143,1.1443,0.33,0.431,0,1,0,0,0,1,1,1,80,0.25,150$

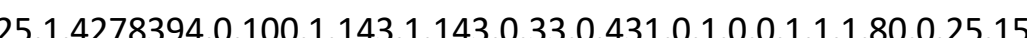
$25,1,4278714,0,112,1.143,1.143,1.397,1.825,0,1,0,0,1,1,1,51,0.25,30$ $25,1,4278396,0,100,1.143,1.143,0.33,0.431,0,1,0,0,0,1,1,1,80,0.25,150$ $25,1,4278221,0,103,1.143,1.1433,1.143,1.493,0,1,0,0,1,1,1,51,0.25,30$

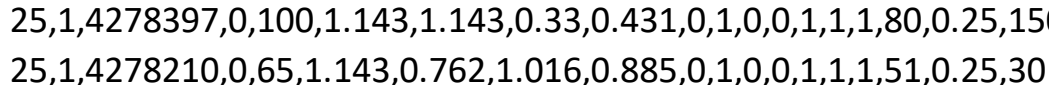
$25,1,4278399,0,100,1.143,1.143,0.33,0.431,0,1,0,0,1,1,1,80,0.25,150$ $25,1,4278218,0,102,1.143,1.1433,1.143,1.493,0,0,1,0,0,1,1,1,51,0.25,30$ $25,1,4278401,0,0100,1.143,1.143,0.33,0.431,0,1,0,0,0,1,1,1,80,0.025,150$ $25,1,4278403,0,000,1.143,1.143,0.033,0.41,0,1,0,0,1,1,1,1,80,0.25,150$ $25,1,4278405,0,100,1.143,1.143,0.33,0.431,0,1,0,0,1,1,1,80,0.25,150$ $25,1,4278407,0,100,1.143,1.143,0.33,0.431,0,1,0,0,1,1,1,80,0.25,150$ $25,1,4278453,0,202,1.143,1.1443,0.381,0.498,0,1,0,0,1,1,1.5,50,0.25,150$

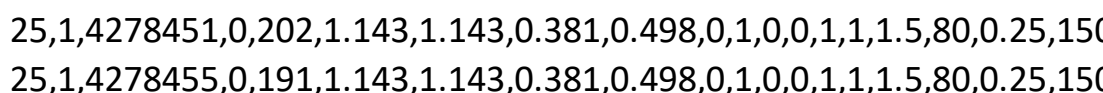
$25,14278456,0,191,1.143,1143,0.3810 .498,0,10,0,1,1,1,580,025,150$ $25,1,4279139,0,037.5,51.143,0.864,0.066,0.652,0,1,1,0,1,1,2,880,0.25,150$ $25,1,4279197,0,306.5,1.067,1.067,0.635,0.0723,0,1,0,0,0,1,1,2,280,0.25,150$ $25,1,4278355,1,1,1.584,0.381,0.05990 .124,0,1,1,0,1,1,1,1,0,0.25,30$ $25,14279124,1995,50.584,0.381,0.559,0.124,0,1,1,01,1,1,51,02530$ $25,1,4278622,1,90.5,0.584,0.381,0.559,0.124,0,1,1,0,1,1,1,51,0.025,30$ $25,1,4278358,0,103,1.016,0.279,0.406,0.115,0,1,1,0,1,1,1,0,80,0.25,150$

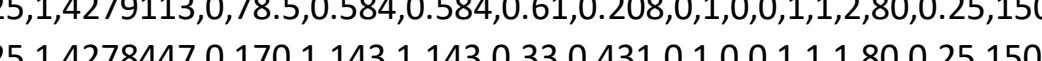
$25,1,4278711,0,107.1 .143,0.762,0.279,0.243,0,1,0,0,1,1,80,0.25,150$ $25,1,4278877,0,133.5,1.143,0.762,0.914,0.796,0,1,0,0,1,1,1,51,0.25,30$ $25,1,42786882,0,0,182.5,1.1433,0.762,0.584,0.0509,0,1,0,0,1,1,1,151,0.25,30$

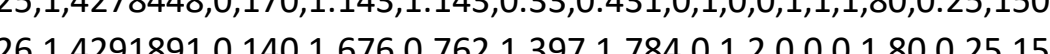
$26,1,4279102,0,136,1,626,0.61,1.346,1.335,0,1,2,0,0,0,1,80,0.25,150$ $26,1,4282179,1,67.5,0.584,0.381,0.559,0.124,0,1,0,1,1,1,51,0.25,30$ $26,1,4282738,1,55.2,0.5844,0.381,0.559,0.124,0,1,1,0,1,1,1,51,0.25,30$ 26, $26,3,4282075,1,1.08,0.18,0.18,0.18,0.006,0,0,0,0,1,1,1,48,0.25,10$

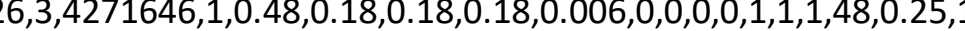
$26,3,4286915,1,0.99,0.18,0.18,0.018,0.006,0,0,0,0,0,1,1,1,48,0,0.25,10$ (26.3. $26,1,4257183,0,0122,1.143,1.143,1.1433,1.493,0,0,1,0,0,1,1,1,51,0.025,30$

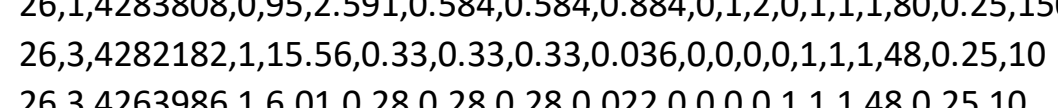

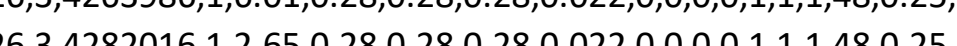

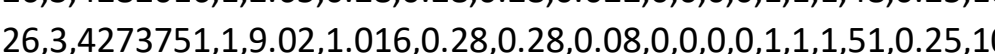

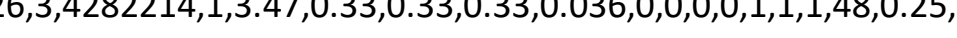
$26,3,4291885,1,7.46,0.28,0.28,0.28,0.0222,0,0,0,0,1,1,1,1,48,0.25,10$ $26,1,4285303,0,01.95,1.651,0.178,0.1022,0.03,0,0,0,0,0,1,1,1,1,32,0.125,10$ $26,4,4290128,0,201,1397,0.178,01520.038,0,0,0,0,1,1,320,125,10$ $26,3,4257159,0,23.74,0.533,0.533,0.33,0.094,0,0,0,0,1,1,1,51,0.25,10$ $26,1,4282090,1,70,0.584,0.0381,0.559,0.124,0,1,1,0,1,1,1,1,51,0.25,30$ $26,3,4282185,1,17.41,0.432,0.432,0.432,0.081,0,0,0,0,0,1,1,1,48,0.025,10$

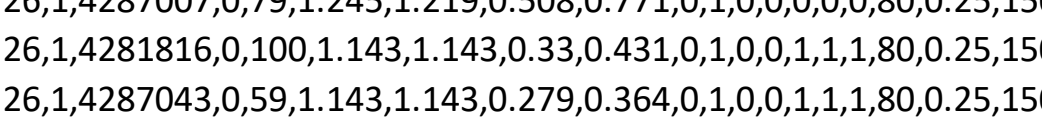


$26,1,4291932,0,123,1.295,0.762,1.27,1.253,0,1,2,0,0,0,0,1,80,0.25,150$ $26,4,4290130,0,20,1.397,0.178,0.152,0.038,0,0,0,0,0,1,1,1,32,0.125,10$ $26,4,4290131,0,20,1.1397,0.178,0.152,0.038,0,0,0,0,1,1,1,1,32,0.125,10$ $26,4,4290133,0,20,1.397,0.178,0.152,0.038,0,0,0,0,0,1,1,1,32,0.0125,10$ $26,3,4283754,1,4.63,0.432,0.432,0.203,0.038,0,0,0,0,0,1,1,51,0,25,10$ $26,3,4282175,1,2.82,0.432,0.432,0.0203,0.038,0,0,0,0,1,1,1,1,51,0.25,10$ $26,4,4290129,0,20,1.397,0.178,0.152,0.038,0,0,0,0,1,1,1,1,32,0.125,10$ $26,1,4279181,028,1.168,0.711,0.1270 .1050,0,0,1,1,1,80,0,25,150$ $26,1,4292944,0,29,1.143,0.66,0.127,0.096,0,1,0,0,0,1,1,1,1,0,0.25,150$ $26,3,4282172,1,12.4,0.0432,0.432,0.203,0.038,0,0,0,0,0,1,1,1,51,0.25,10$ $26,1,4275245,1,198,1.143,0.762,0.0333,0.464,0,1,0,0,0,1,1,1,51,0.25,30$

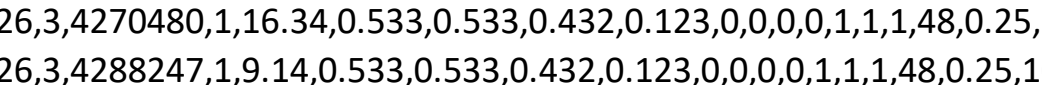
$26,1,4257086,0,183,1.143,1.143,1.295,1.692,0,0,0,0,1,1,1,51,0.25,30$ $26,1,4282197,1,95.5,1.143,1.143,0.965,1.261,0,1,0,0,1,1,1,51,0.25,30$ $26,1,4282178,1,37.6,0.61,0.61,0.666,0.246,0,1,0,0,0,1,1,1,151,0.25,30$

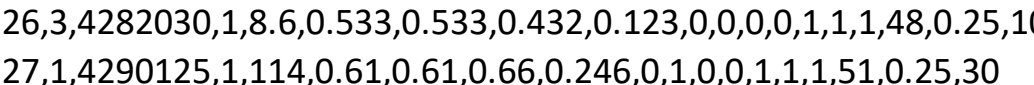
$27,1,4252350,178.20 .610 .0610 .666,2.246,0,0,0,1,1,1,151,0.25,30$ $27,1,4252345,1,44.8,8,61,0.61,0.66,0.246,0,0,0,0,1,1,1,51,0.25,30$ $27,3,4289523,1,4.21,0.33,0.33,0.33,0.036,0,0,0,0,0,1,1,1,48,0.25,10$

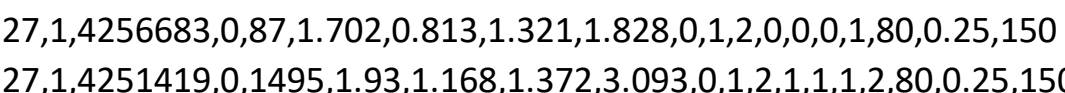
$27,1,4298727,0,169,1.956,0.864,0.381,0.644,0,1,0,0,1,1,1,80,0.25,150$ $27,1,4290114,0,297,1.041,1.016,0.432,0.457,0,1,0,0,1,1,1,80,0.025,150$ $27,3,4258214,1,17.28,0.0635,0.6355,0.432,0.174,0,0,0,0,0,1,1,1,48,0.25,10$ $27,1,4278664,0,0251,2.184,0.66,04$ $27,1,4290056,1,44.6,0.61,0.61,0.66,0.246,0,1,0,0,1,1,1,51,0.25,30$ $27,1,4290088,1,37,0.61,0.61,0.66,0.246,0,1,0,0,1,1,1,51,0.25,30$ $27,1,4255255,0,59,1.016,0.279,0.406,0.1155,0,1,0,0,0,1,1,151,0.25,10$

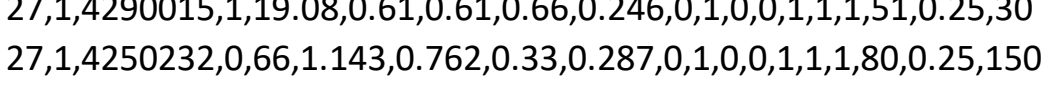
$27,1,4250233,0,66,1.143,0.762,0.33,0.287,0,1,0,0,1,1,1,80,0.25,150$ $27,1,42492244,1,213,1.143,0.762,0.5333,0.464,0,1,0,0,1,1,1,1,51,0.25,30$ $27,3,4290059,127,40,6350,035,0432,0644,0,0,0,0,1,1,1,0,0,30$ $27,3,4290058,19.02,0.33,0.33,0.33,0.036,0,0,0,0,0,1,1,48,0.25,10$ $27,3,4249239,1,22.95,0.432,0.432,0.432,0.081,0,0,0,0,1,1,1,48,0.25$,

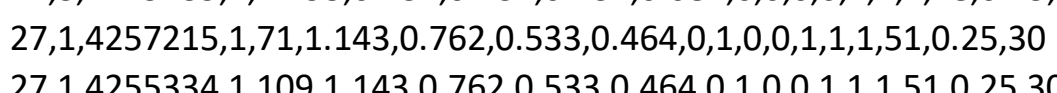
$27,1,2720960,236,1321,12451206,16710,1,0,0,1,1,1,0,0.25,30$

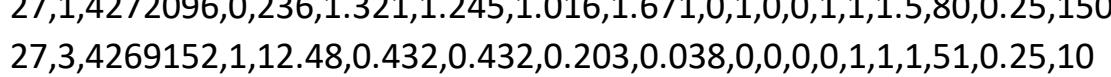
$27,3,4252334,1,7.47,0.432,0.432,0.203,0.038,0,0,0,0,0,1,1,1,51,0.25,10$ $27,3,4269038,1,7.23,0.432,0.432,0.203,0.038,0,0,0,0,0,1,1,1,151,0.25,10$ (27)

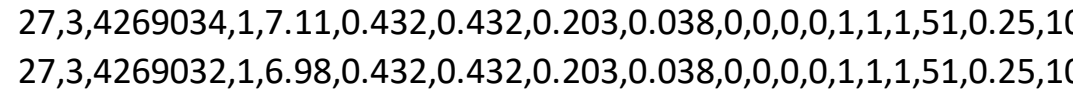

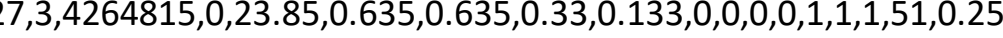
$27,3,42449054,1,6.38,0.28,0.28,0.28,0.0222,0,0,0,0,0,1,1,1,48,0.25,110$ $27,3,4255074,1,2.41,0.28,0.28,0.28,0.022,0,0,0,0,0,1,1,1,4,0.25,10$

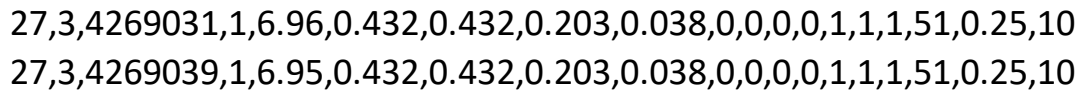

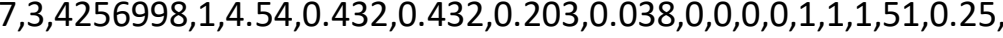
$27,3,4255232,1,0.6,0.018,0.18,0.18,0.006,0,0,0,0,1,1,1,48,0.25,10$ $27,3,4258596,1,0.57,0.18,0.18,0.18,0.000,0,0,0,0,0,1,1,1,1,48,0.025,10$

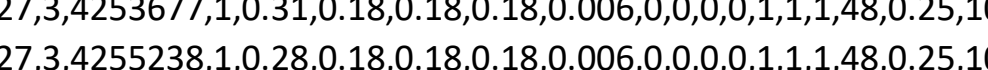
$27,1,4248960,0,0239,1.194,0.787,0.889,0.835,0,1,2,0,1,1,1,80,0.25,150$ $27,1,4269303,1,53,1.143,0.762,0.533,0.464,0,0,1,0,0,1,1,1,51,0.25,30$ $27,3,4253640,1,8.73,0.635,0.635,0.432,0.174,0,0,0,0,0,1,1,1,48,0.25,10$
$27,3257072,122,990.533$

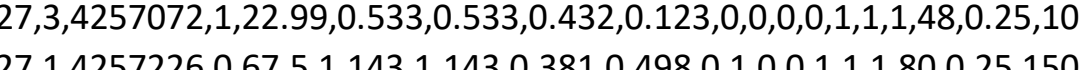
$27,1,4255079,0,54,1.143,1.143,0.584,0.763,0,1,0,0,1,1,1,80,0.25,150$ $27,1,42572220,0,68.5,1.1433,1.143,0.457,0.597,0,1,0,0,1,1,1,80,0.25,150$ $27,1,4252348,1,132.4,1.143,0.762,0.533,0.464,0,1,0,0,0,1,1,1,51,0.25,30$

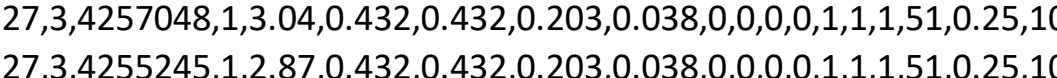
$27,1,4291882,0,011,1.143,0.762,0.737,0.642,0,1,0,0,1,1,1,51,0.25,30$ $27,1,4298675,0,113,0.711,0.0711,0.203,0.103,0,1,0,0,0,1,1,1,80,0.25,150$ $27,1,4255030,0,48,0.66,0.06,0.813,0.354,0,1,0,0,0,1,1,1,80,0.25,150$

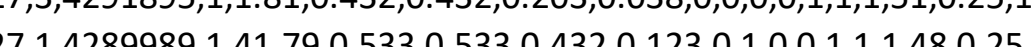
$27,1,4290071,1,45.2,0.584,0.381,0.559,0.124,0,1,1,0,1,1,1,51,0.25,30$ 列, $3,4264703,1,11.73,0.432,0.432,0.0432,0.081,0,0,0,0,1,1,1,48,0.25,10$ $27,3,4255950,1,7.11,0.533,0.406,0.4066,0.088,0,0,0,0,0,1,1,1,48,0.25,10$

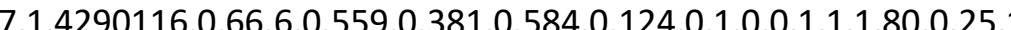
$27,3,4267850,1,1.84,0.33,0.33,0.33,0.036,0,0,0,0,0,1,1,1,148,0.25,10$ $28,1,4277239,1,164,1.143,0.762,0.533,0.0464,0,1,1,0,0,1,1,1,51,0.25,30$ $28,1,42523877,1,66,1.143,0.762,0.965,0.84,0,0,1,0,0,1,1,1,1,51,0.25,30$ $28,1,4255180,1,97,1,143,1,143,0.0651,261,0,1,0,01,1,1,51,0.25,30$ $28,1,4252389,0,116,1.143,1.143,1.321,1.726,0,1,0,0,1,1,1,51,0.25,30$ $28,1,4252400,0,167,1.143,1.143,1.575,2.058,0,0,1,0,0,1,1,1,51,0.25,30$

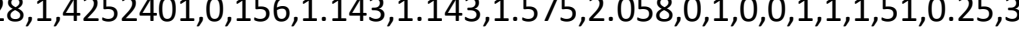
$28,1,4277222,1,57,0.584,0.381,0.559,0.124,0,1,1,0,1,1,1,51,0.25,30$
$28,1,4297722,1,1,57,0.584,0.381,0.559,0.124,0,1,1,0,1,1,1,51,0.25,30$ $28,1,4297848,1,34.20 .5844,0.381,0.559,0.124,0,1,1,0,1,1,51,0.25,30$ $28,1,4277264,1,42.2,0.584,0.381,0.965,0.215,0,1,1,0,1,1,1,51,0.025,30$ $28,1,4255346,1,46.4,0.584,0.381,0.059,0.124,0,1,1,1,1,1,1,51,0.25,30$

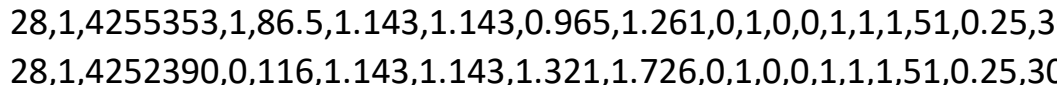

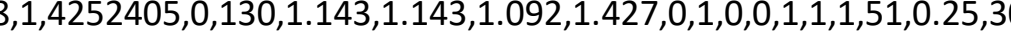
$28,1,4255348,0,93,1.143,1.143,1.092,1.427,0,1,0,0,0,1,1,1,51,0.25,30$ $28,1,4255352,0,111,1.1433,1.143,1.321,1.72726,0,1,0,0,1,1,1,1,51,0.25,30$

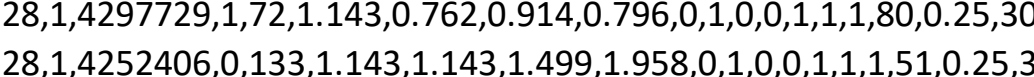
$28,3.42553311,16.95,0.432,0.432,0.432,0.081,0,0,0,0,1,1,48,0.25,10$ $28,3,4277266,1,26.18,0.432,0.432,0.203,0.038,0,0,0,0,0,1,1,1,1,51,0.25,10$ $28,3,4269030,1,7.33,0.432,0.432,0.203,0.038,0,0,0,0,0,1,1,1,51,0.25,10$ 年,

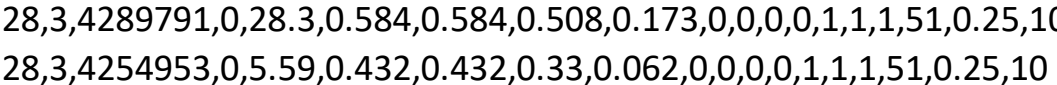
$28,1,4277320,0,42.2,0.66,0.66,0.813,0.354,0,1,0,0,1,1,1,80,0.25,150$ $28,1,4291881,1,79,0.61,0.61,0.66,0.246,0,1,0,0,0,1,1,1,51,0.25,30$

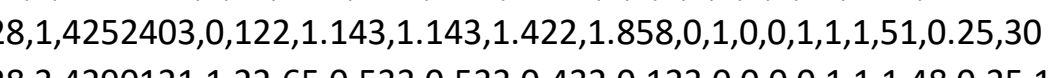

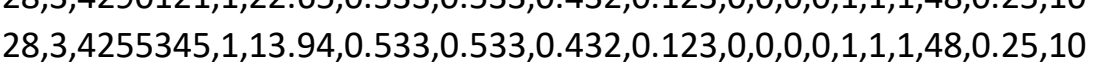
$28,3,4258415,1,12.58,0.533,0.533,0.432,0.123,0,0,0,0,1,1,1,1,48,0.25,10$ $28,3,4297873,1,27.85,0.432,0.432,0.432,0.081,0,0,0,0,0,1,1,1,48,0.25,10$ 年, $29,1,4294602,0,193,1.143,0.762,0.279,0.243,0,1,0,0,0,1,1,1,1,0,0.25,150$ $29,1,4294614,0,104.5,1.143,0.762,0.559,0.487,0,1,0,0,1,1,1,51,0.25,30$ $29,1,4294307,0,267,1.143,1.143,0.381,0.498,0,1,0,0,1,1,1,1,80,0.25,150$

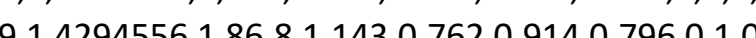

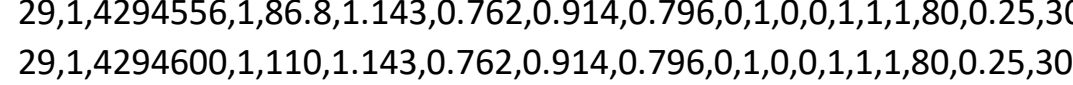

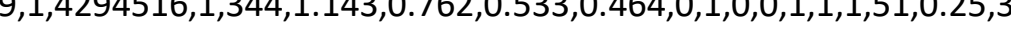
$29,1,4294310,0,1911,1.143,1.143,0.381,0.498,0,1,0,0,1,1,1,180,0.25,150$ $29,1,4294223,0,103,1.143,1.143,1.168,1.526,0,0,0,0,1,1,1,1,51,0.025,30$ $29,1,4294316,0,180,1.143,1.143,0.381,0.498,0,0,1,0,0,1,1,1,80,0.25,150$ $29,1,4294222,0,033,1.143,1.143,1.168,1.526,0,1,0,0,0,1,1,1,51,0.25,30$ 年, $29,1,42943250,12411431.14303330 .4310,10,01,1,1,80,025,150$ $29,1,4294330,0,124,1.143,1.143,0.33,0.431,0,10,0,1,1,1,80,0.25,150$ $29,1,4294519,1,103,0.61,0.61,0.66,0.246,0,1,0,0,1,1,1,51,0.25,30$ $29,1,4294318,0,180,1.143,1.143,0.0381,0.498,0,1,0,0,0,1,1,1,80,0.25,150$ $29,1,4294322,0,0,124,1.143,1.143,0.33,0.431,0,1,0,0,0,1,1,1,1,80,0.25,150$ $29,1,4294328,0,124,1.143,1.143,0.33,0.431,0,1,0,0,1,1,1,180,0.25,150$ 
$29,1,4294331,0,124,1.143,1.143,0.33,0.431,0,1,0,0,0,1,1,1,80,0.25,150$ $29,1,4294552,1,78,0.584,0.381,0.559,0.124,0,0,1,1,0,1,1,1,151,0.25,30$ $29,1,4294337,0,1166,0.965,0.0965,1.168,1.088,0,1,0,1,1,1,2,2,80,0.25,150$

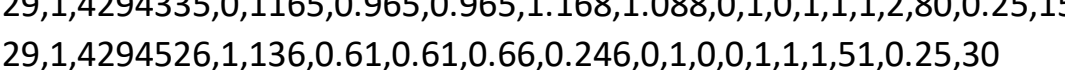
$29,1,4294339,0,095,0.889,0.559,1.041,0.517,0,1,0,0,1,1,5,50,0.25,150$ $29,1,4294613,1,45,1.143,0.762,0.965,0.84,0,1,0,0,1,1,1,151,0.25,30$ $29,1,4294295,0,318,1.143,1.143,0.0381,0.498,0,1,0,0,0,1,1,1,80,0.25,150$

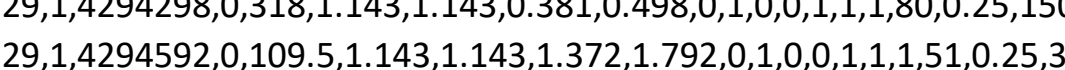
$29,1,4294297,0,318,1.143,1.143,0.381,0.498,0,1,0,0,1,1,1,80,0.25,150$ $29,1,4294299,0,318,1.143,1.143,0.381,0.498,0,1,0,0,1,1,1,80,0.25,150$ $29,1,4294605,0,109,1.143,1.143,1.372,1.792,0,1,0,0,1,1,1,1,51,0.25,30$ 30,14297492, $0104.1 .93,1.533,0.359,0.595,0,1,0,0,1,1,1,80,0.25,150$ $30,1,4305025,0,100,1.143,1.143,0.33,0.431,0,1,1,0,0,1,1,1,1,0,0.25,150$ $30,1,4290118,0,236,1.143,1.143,1.295,1.692,0,1,0,0,1,1,1,51,0.25,30$

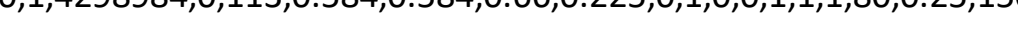

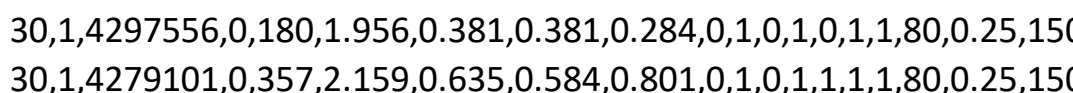
$30,1,4290075,0,125,1.143,0.752,1.27,1.106,0,1,0,0,1,1,1,51,0.25,30$ $30,4,4305186,0,14,1.194,0.533,0.178,0.113,0,0,0,0,0,1,1,1,51,0.25,10$ $30,1,4297482,0,100,1.143,1.143,0.33,0.431,0,0,1,0,0,1,1,1,1,180,0.25,150$

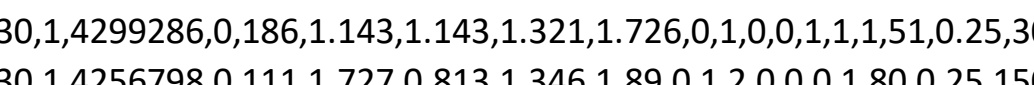
$30,1,4297484,0,100,1.143,1.143,0.33,0.431,0,1,0,0,1,1,80,0.25,150$ $30,1,4299258,0,208,1.143,1.143,1.295,1.692,0,1,0,0,0,1,1,1,51,0.025,30$ $30,1,4305749,1,79,1.143,0.0762,0.533,0.4644,0,1,0,0,0,1,1,1,51,0.25,30$ (3), $30,3,4299407,1,20.28,0.432,0.432,0.203,0.038,0,0,0,0,0,1,1,1,151,0.25,10$ $30,3,4302707,1,15.45,0.787,0.203,0.203,0.032,0,0,0,0,1,1,1,1,48,0.25,10$ $30,3,4304033,1,6.01,0.787,0.203,0.203,0.032,0,0,0,0,1,1,1,148,0.25,10$ $30,3,4302706,1,1,31,0.432,0.432,0.432,0.0 .081,0,0,0,0,0,1,1,1,48,0.25,10$

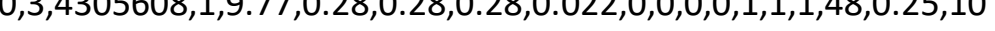
$30,3,4297751,1,1.47,0.28,0.28,0.28,0.022,0,0,0,0,0,1,1,1,48,0.25,10$

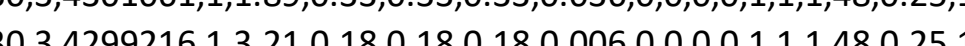
$30,3,4307350,1,0.93,0.18,0.18,0.18,0.006,0,0,0,1,1,1,48,0.25,10$ $30,3,4300999,1,0.46,0.18,0.18,0.18,0.006,0,0,0,0,1,1,1,18,0.25,10$ $30,3,4299213,1,1.07,0.18,0.18,0.18,0.006,0,0,0,0,0,1,1,1,48,0.25,10$ 年,

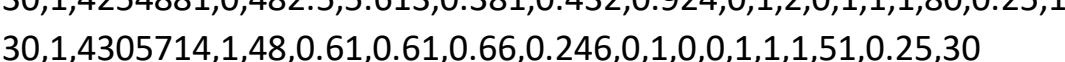
$30,1,4305724,1,62.6,0.584,0.381,0.559,0.124,0,0,1,1,1,1,1,1,51,0.25,30$

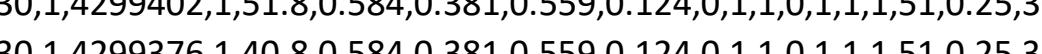
$31,1,43183911,1,91,0.584,0.381,0.5590,0.124,0,1,1,0,1,1,51,0.25,30$ $31,1,4318770,0,069,1.143,0.762,1.372,1.195,0,1,0,0,1,1,1,151,0.25,30$ $31,1,43182055,0,645,1.676,0.8388,1.245,1.749,0,1,0,0,0,1,1,1,80,0.25,150$

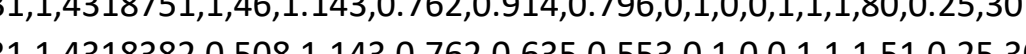
$31,1,43181990,0,106,1.143,0.762,0.279,0.243,0,0,0,0,1,1,180,0.25,150$ $31,1,4318063,0,119,1.143,1.143,1.143,1.493,0,1,0,0,1,1,1,51,0.25,30$ $31,1,4318124,0,0603,3.708,0.635,0.559,1.316,0,1,0,0,1,1,1,1,80,0.25,150$ $31,1,43184244,1,32,0.61,0.61,0.66,0.0246,0,1,0,0,1,1,1,51,0.25,30$

$31,1,4318638,0,111,1.143,1.143,1.422,1.858,0,1,0,0,1,1,1,51,0.25,30$ $31,1,4318473,1,1,6,1.1433,0.762,0.533,0.464,0,1,0,0,1,1,1,51,0.25,30$ $31,1,4318593,0,111,1.143,1.143,1.397,1.825,0,1,0,0,1,1,1,15,0.25,30$ $31,1,4318648,1,169,1.143,1.143,0.965,1.261,0,1,0,0,0,1,1,1,51,0.25,30$

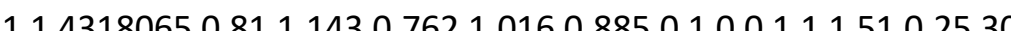
$32,1,4320489,0,43,1.143,1.143,0.406,0.53,0,1,0,0,1,1,1,80,0.25,150$ $32,1,4340125,0,109,1.143,1.143,0.33,0.431,0,1,0,0,1,1,1,80,0.25,150$ $32,1,431786,0,44,0.762,1.168,0.279,0.248,0,1,0,0,1,1,1,80,0.25,150$ $32,14340372,0,170,1.93,0.864,0.381,0.635,0,1,0,0,1,1,180,0.25,150$ $32,1,4340005,0,118,1.93,0.61,0.686,0.808,0,1,0,0,1,1,1,80,0.25,150$ $32,1,4340236,0,113,0.711,0.711,0.203,0.0103,0,1,1,0,0,1,1,1,80,0.25,150$ $32,1,4344319,0,0,180,1.956,0.0305,0.406,0.242,0,1,0,0,0,1,1,1,80,0.25,150$ $32,13457960.1964,20570.1520 .0102,0032,0,0,0,1,1,1,320,1250$ $32,1,4317214,0,59,1.143,1,143,0279,0364,0,1,0,0,1,1,1,80,0.25,150$ $32,1,4340745,1,300,1.143,0.762,0.533,0.464,0,1,0,0,1,1,1,51,0.25,30$ $32,3,43172222,0,19.7,0.6355,0.635,0.356,0.144,0,0,0,0,0,1,1,1,51,0.25,10$

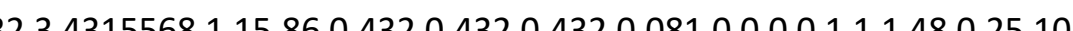

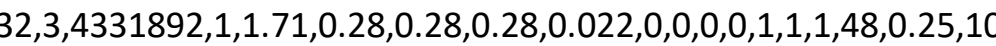
$32,3,4315432,1,1.34,0.28,0.28,0.28,0.0222,0,0,0,0,1,1,1,48,0.25,10$ $32,1,4327141,0,82,1.219,1.219,0.559,0.8311,0,1,0,0,0,1,1,1,80,0.25,150$ $32,3440605,1632,0280,28,0280,0220,0,0,0,1,1,48,0,25,10$ $32,3,4317273,1,2.98,0.28,0.28,0.28,0.022,0,0,0,0,0,1,1,1,48,0.25,10$ $32,3,4318974,1,1.17,0.28,0.028,0.28,0.022,0,0,0,0,0,1,1,1,148,0.25,10$ $32,3,4315595,1,2.19,0.18,0.18,0.18,0.006,0,0,0,0,0,1,1,1,48,0.25,10$

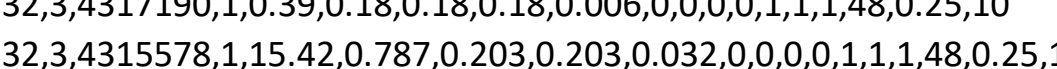
$32,3,4317200,1,13.94,0.787,0.203,0.203,0.032,0,0,0,0,0,1,1,1,48,0.25,10$ $32,3,4340685,1,13.14,0.787,0.203,0.203,0.032,0,0,0,0,1,1,1,148,0.25,10$ $32,3,4320521,1,10.36,0.432,0.432,0.203,0.038,0,0,0,0,0,1,1,1,51,0.25,10$ $32,3,4315537,1,3.05,0.432,0.432,0.023,0.038,0,0,0,0,0,1,1,1,51,0.25,10$ $32,3,4340726,1,18.8,0.432,0.432,0.432,0.081,0,0,0,0,0,1,1,1,48,0.25,10$ $32,3,4320517,1,16.43,0.33,0.33,0.33,0.036,0,0,0,0,0,1,1,1,48,0,0.25,10$ $32,3,4340753,1,14.49,0.33,0.33,0.33,0.036,0,0,0,0,0,1,1,1,48,0.25,10$ 年, $32,1,4315120,0,94,2.87,0.533,0.457,0.699,0,1,0,0,1,1,80,0.25,150$ $32,1,4339995,0,94,2.921,0.559,0.457,0.746,0,1,0,0,0,1,1,1,80,0.25,150$ $32,1,4315253,1,197,1.143,0.762,0.533,0.464,0,0,0,0,0,1,1,1,1,1,0.25,30$ 年, $32,1,4330452,1,153.5,1.143,0.762,0.533,0.464,0,1,1,0,01,1,151,0.25,30$ $32,3,4344199,0,9.65,0.0635,0.635,0.356,0.144,0,0,0,0,0,1,1,1,51,0.25,10$ $32,3,4315346,1,4.5,0.432,0.0432,0.432,0.081,0,0,0,0,1,1,1,48,0.25,10$

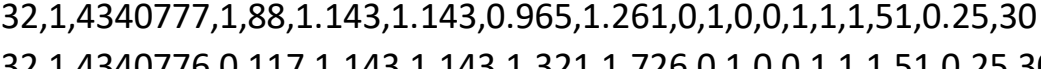
$32,1,4340731,0,157,0.914,0.914,0.279,0.233,0,1,0,0,0,1,1,1,180,0.25,150$ $32,1,4315571,1,53,0.61,0.61,0.66,0.246,0,0,0,0,1,1,1,151,0.25,30$ $32,1,4340748,1,69,0.584,0.381,0.559,0.124,0,1,1,0,1,1,1,51,0.25,30$ $33,1,4360310,0,0,47,2.438,2.3 .37,0.58,28.84,0,1,2,1,1,1,1,80,0.25,150$ $33,3,4360542,1,2.06,0.28,0.28,0.28,0.022,0,0,0,0,0,1,1,1,1,48,0.25,10$ $33,3,4360587,1,8.22,0.432,0.432,0.432,0.081,0,0,0,0,0,1,1,1,48,0.25,10$ $33,1,4360662,0,54,0.762,1.143,1.448,1.261,0,1,0,0,0,1,1,1,80,0.25,150$

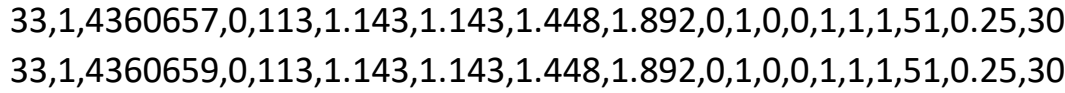
$3,1,4360496,0,112,1.143,1.143,1.448,1.892,0,1,0,0,1,1,1,51,0.25,30$ $33,1,4360500,0,112,1.1443,1.143,1.448,1.892,0,0,1,0,0,1,1,1,51,0.25,30$ (3),

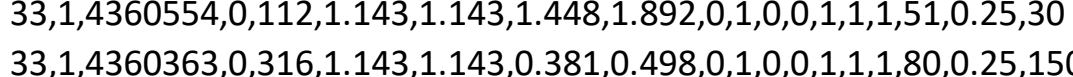
$33,1,4360360,0,187,1.143,1.143,0.381,0.498,0,1,0,0,1,1,1,00,0.25,150$ $33,1,4360740,0,166,1.143,1.143,1.549,2.024,0,1,0,0,1,1,1,51,0.25,30$ (3) $33,1,4360569,0,0105,0.762,1443,0305,02560,0,0,0,1,1,1,0,025,150$ $33,1,4360859,0,94,0.762,1.143,0.086,0.0597,0,1,0,0,1,1,1,1,8,0,0.25,150$ $33,1,4361042,0,87,0.762,1.143,0.686,0.597,0,1,0,1,1,1,80,0.25,150$

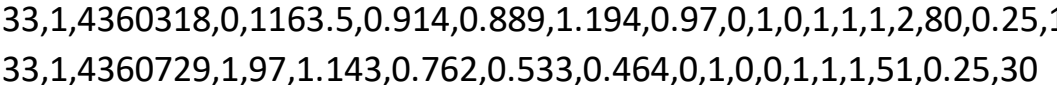

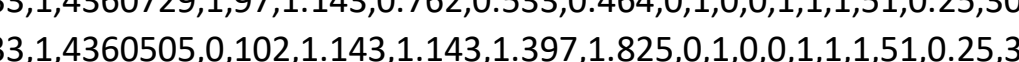
$33,1,4360732,0,55,0.635,0.635,0.838,0.338,0,1,0,0,1,1,1,18,0.25,150$ 
$33,1,4360890,1,157,0.61,0.61,0.66,0.246,0,1,0,0,1,1,1,51,0.25,30$ $33,1,4360892,1,465,1.143,0.762,0.965,0.844,0,1,0,0,1,1,1,1,1,0.0 .25,30$ $34,1,4361317,0,120,1.143,1,1.143,0.33,0.0431,0,1,0,0,1,1,1,1,80,0.25,150$

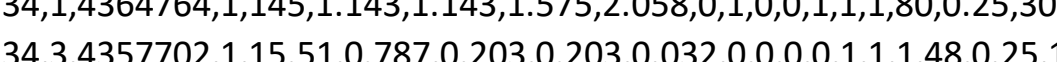
$34,3,3352745,1,10.72,0.787,0.203,0.203,0.032,0,0,0,0,0,1,1,1,48,0.25,10$ $34,3,4352652,0,11.61,0.635,0.635,0.127,0.051,0,0,0,0,0,1,1,1,51,0.25,10$ $34,3,4350824,0,9.938,0.635,0.0635,0.127,0.051,0,0,0,0,0,1,1,1,51,0.25,10$ $34,14364765,1144.1414311431,575,2050,0,1,0,1,1,80.25,150$ $34,3,4364616,1,1.72,0.28,0.28,0.28,0.022,0,0,0,0,1,1,1,48,0.25,10$ $34,3,4352657,1,1.34,0.28,0.28,0.28,0.022,0,0,0,0,1,1,1,48,0.25,10$ $34,3,4361056,1,1.35,0.028,0.28,0.28,0.0222,0,0,0,0,0,1,1,1,1,48,0.25,10$

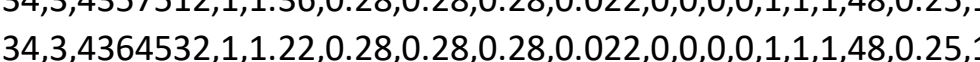
$34,3,4365551,1,1.110 .028,0.28,0.28,0.022,0,0,0,0,1,1,1,48,0.25,10$ $34,3,4359433,1,1.12,0.28,0.28,0.28,0.022,0,0,0,0,1,1,1,48,0.25,10$ $34,1,4355825,0,36.6,0.584,0.381,0.635,0.141,0,1,0,0,0,1,1,1,51,0.25,30$

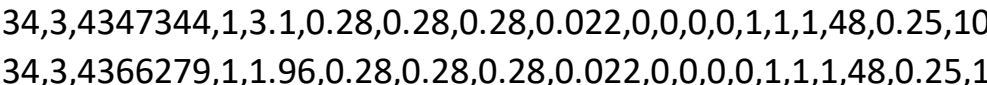
$34,3,4352241,1,1,52,0.28,0.28,0.28,0.022,0,0,0,0,1,1,1,48,0.25,10$ $34,1,4357611,0,298,1.016,1.016,0.432,0.446,0,1,0,0,1,1,1.5,8,0,0.25,150$ $34,1,4357147,0,232,0.991,1.905,0.889,1.678,0,1,1,2,0,1,1,2,2,80,0.25,150$ 年,

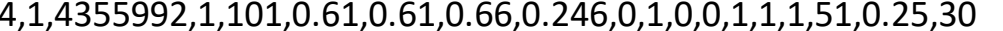
$34,1,4363972,0,21.5,2.235,0.33,0.33,0.243,0,0,0,0,1,1,1,1,51,0.25,10$ $34,1,4357565,1,97,0.584,0.381,0.559,0.124,0,1,1,0,1,1,1,151,0.25,30$

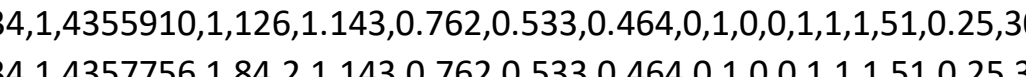
$34,4,4352744,1,1,42,1,1.245,0.127,0.127,7,0.02,0,0,0,0,0,1,1,1,1,32,0.125,10$ $34,4,4351014,1,1,522,1.245,0.127,0.127,0.02,0,0,0,0,1,1,1,1,32,0.125,10$ $34,1,4357715,1,96,0.584,0.3831,0.559,0.124,0,1,1,0,0,1,1,1,151,0.25,30$ $34,1,4357181,0,246,0.61,0.011,0$

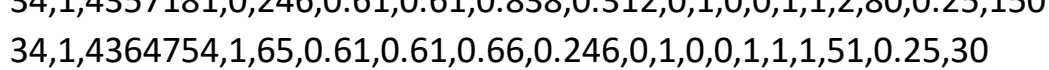
(4), $1,4356028,1,44.8,0.61,0.61,0.66,0.246,0,1,0,0,1,1,1,51,0.025,30$ $34,1,4361335,1,79,0.584,0.381,0.559,0.124,0,1,1,0,0,1,1,1,51,0.25,30$ 34,1,4366297,1,0,0.584,038

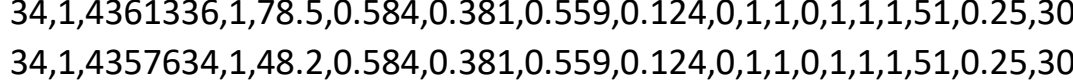
$34,1,4355940,1,42,0.584,0.0381,0.559,0.124,0,1,1,0,1,1,1,51,0.25,30$ $34,3,4354075,1,7.92,0.33,0.33,0.33,0.036,0,0,0,0,1,1,1,48,0.25,10$

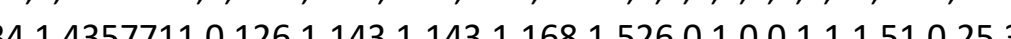

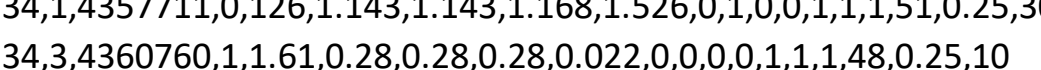
$34,3,4357728,1,13.67,1.016,0.28,0.28,0.08,0,0,0,0,0,1,1,1,51,0.25,10$ $34,3,4347423,1,13.17,1.016,0.28,0.28,0.08,0,0,0,0,0,1,1,1,51,0.25,10$

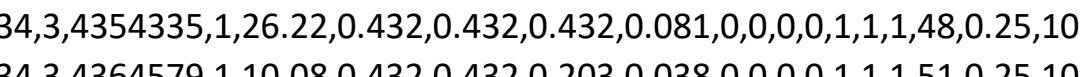

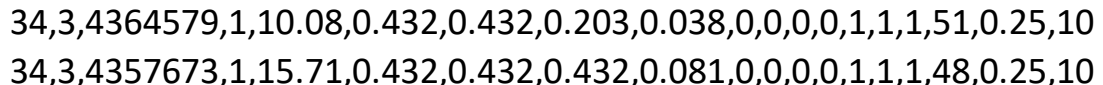
$34,3,4364716,1,1,99,0.432,0.432,0.203,0.038,0,0,0,0,0,1,1,1,51,0.25,10$ $34,3,4364518,0,7.39,0.533,0.533,0.127,0.036,0,0,0,0,1,1,1,51,0.25,10$

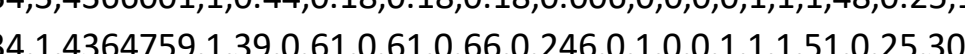

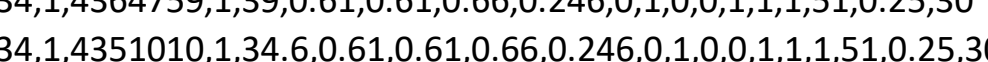
$34,1,4357539,0,056,0.61,0.457,0.483,0.135,0,1,1,0,1,1,0.5,80,0.25,150$ $34,3,4357612,1,19.32,0.533,0.533,0.432,0.123,0,0,0,0,0,1,1,1,48,0.25,10$ $34,1,4366296,1,70,1.143,0.762,0.533,0.464,0,0,1,0,0,1,1,1,1,51,0.25,30$

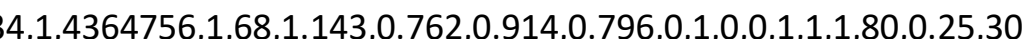
$34,1,4364760,0,67,1.143,0.762,0.686,0.597,0,1,0,0,1,1,1,15,0.25,30$ $34,3,4352762,1,13.73,0.533,0.533,0.432,0.123,0,0,0,0,0,1,1,1,48,0.25,10$ $34,3,4351087,1,9.92,0.0533,0.533,0.432,0.123,0,0,0,0,0,1,1,1,48,0.25,10$ $34,14352535,150,1.143,0.762,05330.4640,1,0,1,1,1,15,02530$ $34,1,4357684,1,94,1.143,1.143,0.965,1.261,0,1,0,0,1,1,1,51,0.25,30$ $34,4,4364332,1,6.98,1.245,0.127,0.127,0.02,0,0,0,0,0,1,1,1,32,0.125,10$ $34,1,4357696,0,95,1.143,1.143,1.041,1.36,0,1,0,0,0,1,1,1,51,0.25,30$

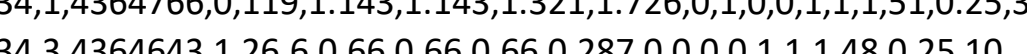
$34,3,4352736,0,7.46,0.61,0.61,0.152,0.057,0,0,0,0,1,1,1,51,0.25,10$ $34,3,4366298,0,8.2 .2,0.432,0.432,0.33,0.062,0,0,0,0,0,1,1,1,51,0.25,10$ $34,3,43528220,1,3.1,1,0.432,0.4322,0.203,0.038,0,0,0,0,0,1,1,1,51,0.25,10$

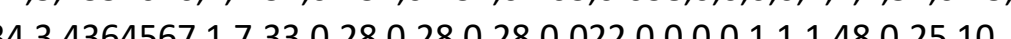
$34,3,3357748,1,7,07,0.28,0.28,0.28,0.0222,0,0,0,0,1,1,1,48,0.25,10$ $34,3,4350906,1,11.28,1.016,0.28,0.28,0.08,0,0,0,0,0,1,1,1,51,0.0 .25,10$ $34,3,4350936,1,8.04,1.016,0.28,0.28,0.08,0,0,0,0,0,1,1,1,51,0.25,10$ $34,34352591,1,539,0.432,0.432,0.2030 .038,0,0,0,1,1,1,51,025,10$ $34,3,4350927,1,5.2,0.432,0.432,0.203,0.038,0,0,0,0,0,1,1,1,51,0.25,10$ $34,3,4362620,1,1.09,0.432,0.432,0.203,0.038,0,0,0,0,1,1,1,51,0.25,10$ $34,3,4352824,1,3.85,0.432,0.432,0.203,0.038,0,0,0,0,0,1,1,1,51,0.25,10$

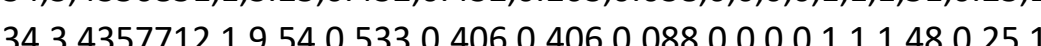
$34,3,4357770,14.83,0.18,0.18,0.18,0.006,0,0,0,0,1,1,1,48,0.25,10$ $34,3,4355728,1,13.44,0.18,0.18,0.18,0.006,0,0,0,0,1,1,1,48,0.025,10$ $34,3,4357637,1,1.1,0.18,0.18,0.18,0.006,0,0,0,0,0,1,1,1,148,0.25,10$

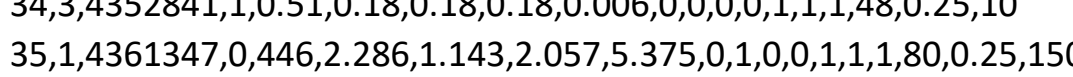
$35,1,4361343,0,398,2.286,1.143,2.057,5.375,0,1,0,0,1,1,1,1,80,0.25,150$ $35,1,4361330,0,99,1.143,0.762,1.194,1.04,0,1,1,0,1,1,1,51,0.25,30$ (3), $35,1,4361351,1,53,1.143,0.762,0.914,0.796,0,1,0,0,0,1,1,1,80,0.25,30$ (5), $1,4361332,0,98.5,1.143,0.762,1.194,1.04,0,1,0,0,1,1,1,151,0.25,300$ $35,1,4361345,0,03,1.143,1.143,1.092,1.427,0,0,1,0,0,1,1,1,51,0.25,30$ $35,1,4361350,0,250,1.143,1.143,1.27,1.659,0,1,0,0,0,1,1,151,0.25,30$ $35,1,4361346,0,111,1.143,141.143,1.0161,1.327,0,1,0,0,1,1,1,1,51,0.25,30$ $35,14361341,0,234,1.143,1.143,1.2451,1627,0,10,0,1,1,51,0.25,30$ $35,1,4361349,0,09,1.143,1.143,0.965,1.261,0,0,1,0,0,1,1,1,151,0.25,30$ $36,3,4324001,1,13.88,0.432,0.432,0.0203,0.038,0,0,0,0,0,1,1,1,51,0.25,10$ $36,3,4315620,1,1.79,0.28,0.28,0.28,0.022,0,0,0,0,1,1,1,1,48,0.25,10$ $36,3,4347325,1,1.84,0.28,0.28,0.28,0.022,0,0,0,0,1,1,1,1,48,0.25,10$ $36,1,43534207,0,250.5,1.1944,0.813,0.533,0.0517,0,1,2,0,1,1,1,2,80,0.25,150$ (36,1,4336568,1,230,1.143,0.762,0.533,0.464,0,1,0,0,1,1,1,51,0.25,30 $36,1,4358913,1,48,1.143,0.762,0.533,0.464,0,1,0,0,1,1,151,0.25,30$ $36,1,4354239,1,7.05,1.143,1.143,0.965,1.261,0,1,0,0,1,1,1,51,0.25,30$ $36,3,4324028,1,9.11,1.016,0.028,0.28,0.08,0,0,0,0,0,1,1,1,151,0.25,10$ 年 $36,3,4355798,1,13.81,0.28,0.28,0.28,0.022,0,0,0,0,1,1,1,48,0.25,10$ $36,3,4371054,1,2.74,0.28,0.28,0.28,0.022,0,0,0,0,1,1,1,148,0.25,10$ $36,3,4323944,1,2.276,0.28,0.28,0.28,0.022,0,0,0,0,1,1,1,1,48,0.25,10$ 年, $36,3,4336580,1,1.89,0.28,0.28,0.28,0.022,0,0,0,0,0,1,1,1,4,0.25,10$ $36,3,4336583,1,1.88,0.28,0.28,0.28,0.022,0,0,0,0,1,1,1,48,0.25,10$ $36,3,4336907,1,11.11,0.33,0.33,0.33,0.036,0,0,0,0,0,1,1,1,48,0.25,10$ (36,

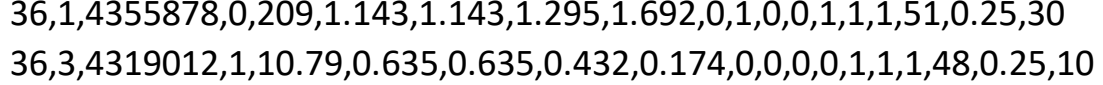
$36,1,4358901,1,40,1.143,0.762,0.533,0.464,0,1,0,0,0,1,1,1,51,0.25,30$ $36,3,4347321,1,23.12,0.432,0.432,0.203,0.038,0,0,0,0,1,1,1,1,51,0.25,10$ 36,3,4347424,1,14.69,0.432,0.432,0.023,0.038,0,0,0,0,0,1,1,1,1,51,0.25,10 $36,34354247,1,6850.4320 .4320 .2030 .038,0,0,0,1,1,51,025,10$ (6), $3,431707,6,1,10.67,0.28,0.28,0.28,0.022,0,0,0,0,0,1,1,1,48,0.25,10$ $36,3,43737036,1,10.03,0.28,0.28,0.28,0.022,0,0,0,0,0,1,1,1,48,0.25,10$ $36,3,4364545,1,6.9,0.028,0.28,0.028,0.022,0,0,0,0,0,0,1,1,1,48,0.25,10$

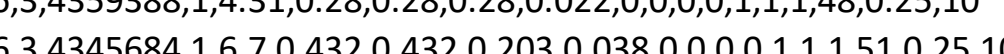
$36,3,4344281,1,6.62,0.432,0.432,0.203,0.038,0,0,0,0,0,1,1,1,51,0.25,10$ 
$36,3,43543331,1,4.46,0.0432,0.432,0.2033,0.038,0,0,0,0,0,1,1,1,51,0.025,10$ 年, $66,3,4355789,1,4.01,0.28,0.28,0.28,0.022,0,0,0,0,1,1,1,4,48,0.25,10$

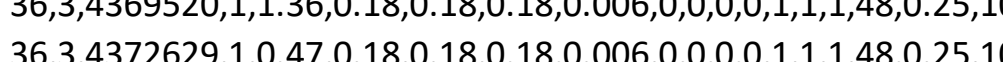
$36,3,4324170,1,0.92,0.18,0.18,0.18,0.006,0,0,0,0,1,1,1,48,0.25,10$ $36,3,4357651,1,0.4,0.18,0.188,0.18,0.006,0,0,0,0,0,1,1,1,48,0.25,10$ $36,3,4336541,1,0.83,0.18,0.18,0.18,0.006,0,0,0,0,1,1,1,1,48,0.25,10$ $36,3336540,1083,0180,18018000600,0,1,1,1,025,10$ $36,3,4372659,1,0.34,0.18,0.18,0.18,0.006,0,0,0,0,0,1,1,1,48,0.025,10$ $36,1,4358910,1,40,1.143,0.762,0.533,0.464,0,1,0,0,1,1,1,51,0.25,30$ $36,1,4358903,1,40,1.143,0.762,0.533,0.464,0,0,1,0,0,1,1,1,1,0,0.25,30$

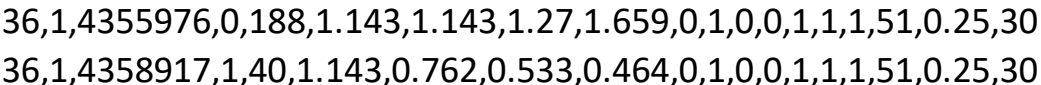
$36,1,4358919,1,40,1.143,0.762,0.533,0.464,0,1,0,0,1,1,1,51,0.25,30$ $36,1,4358918,1,40,1.143,0.762,0.533,0.464,0,1,0,0,1,1,1,51,0.25,30$ $36,1,4372628,0,166,1.143,1.143,1.549,2.2024,0,0,1,0,0,1,1,1,51,0.25,30$

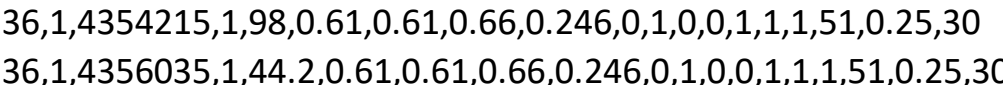
$36,1,4372638,0,551.143,0.762,0.711,0.619,0,1,0,0,1,1,51,0.25,30$ $36,1,4355982,1,103,1.143,0.762,0.965,0.84,0,1,0,0,1,1,1,51,0.25,30$ $36,1,4355980,1,101,1.143,0.762,0.965,0.844,0,1,0,0,1,1,1,51,0.25,30$ $36,1,4355975,1,39.6,6,0.61,0.61,0.66,0.246,0,0,0,0,0,1,1,1,51,0.25,30$ $36,4,43254750,20,1245,0.178,0203500450,0,0,011,1,232,125,10$ $36,1,4344326,1,32,0.584,0.381,0.965,0.215,0,1,1,0,1,1,1,51,0.25,30$ $36,1,4317003,1,30,0.584,0.381,0.559,0.124,0,1,1,0,1,1,1,51,0.25,30$

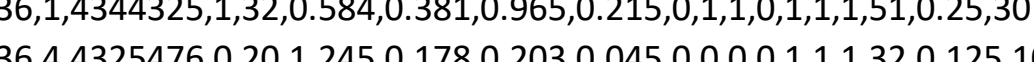
$36,3,4354165,1,6,67,0.33,0.33,0.33,0.036,0,0,0,0,1,1,1,48,0,25,10$ $36,1,4353939,1,53,1.143,0.762,0.533,0.464,0,1,0,0,1,1,1,51,0.25,30$ $36,1,43539363,1,47,1.143,0.762,0.5333,0.464,0,1,0,0,0,1,1,1,151,0.25,30$

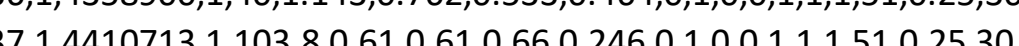

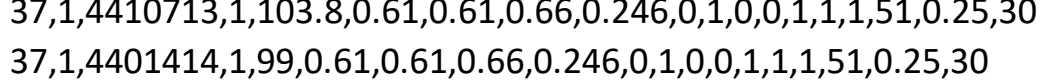
$37,1,4401490,1,52.6,0.61,0.01,0.66,0.246,0,1,0,0,1,1,1,51,0.25,30$ $37,1,4405424,1,94,0.61,0.61,0.66,0.246,0,1,0,0,0,1,1,1,51,0.25,30$

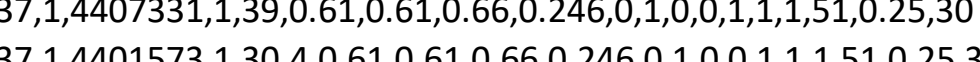
$37,4,4417499,0,13.6,1.448,0.178,0.178,0.046,0,0,0,0,0,0,1,1,0,32,0.125,10$ $37,1,4405352,1,196,1.143,0.762,0.533,0.464,0,0,0,0,0,1,1,1,51,0.25,30$ $37,1,4405354,1,196,1.143,0.762,0.533,0.464,0,0,0,0,0,1,1,1,51,0.25,30$ 年, $37,1,4406383,1,132,1.143,0.762,0.533,0.464,0,0,0,0,0,1,1,151,0.25,30$ $37,1,4405350,1,130,1.143,0.762,0.533,0.464,0,1,0,0,1,1,1,51,0.25,30$ $37,1,4426017,0,68,1.143,0.762,1.067,0.929,0,1,0,0,0,1,1,1,51,0.25,30$

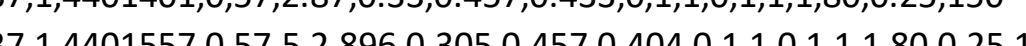
$37,1,4401557,0,57.5,2.2896,0.305,0.457,0.404,0,1,1,0,1,1,1,180,0.25,150$
$37,1,4410562,0,26,2.210 .0305,0.305,0.206,0,0,0,0,1,1,0,32,0.125,10$ $37,3,4401382,1,15.54,0.635,0.635,0.432,0.174,0,0,0,0,1,1,1,48,0.25,10$ $37,3,4401423,1,9.18,0.635,0.635,0.432,0.174,0,0,0,0,0,1,1,1,148,0.25,10$ $37,1,4426059,0,144,1.219,1.219,1.397,2.076,0,0,0,0,0,0,0,0,0,80,0.25,100$

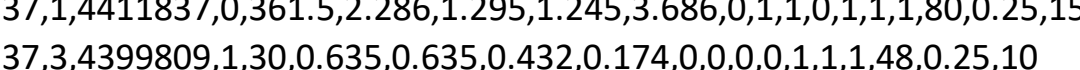

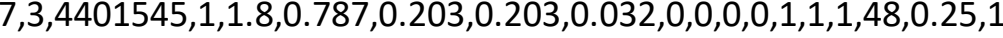
$37,3,4412841,1,12.98,0.432,0.432,0.203,0.038,0,0,0,0,1,1,1,151,0.25,10$ $37,3,4410648,1,4.16,0.432,0.432,0.203,0.038,0,0,0,0,0,1,1,1,51,0.25,10$

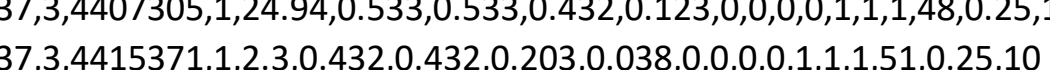
$37,3,4399800,1,19.5,0.635,0.635,0.432,0.174,0,0,0,0,1,1,1,48,0.25,10$ $37,3,4403236,1,2.19,0.28,0.28,0.28,0.022,0,0,0,0,0,1,1,1,48,0.25,10$ $37,3,403257,1,1.27,0.28,0.22,0.28,0.0222,0,0,0,0,0,1,1,1,148,0.025,10$ (3) $37,1,4405387,0,54,1.143,1.143,0.457,0.597,0,1,0,0,1,1,1,1,80,0.25,150$ $37,1,4415452,1,164,1.143,0.0762,0.914,0.796,0,1,0,0,0,1,1,1,80,0.25,30$ $37,1,4425998,0,53,1.143,0.762,0.0635,0.553,0,1,0,0,0,1,1,1,51,0.25,30$ 年, $37,3,4423981,1,3.81,0.432,0.432,0.0203,0.038,0,0,0,0,0,1,1,1,51,0.25,10$ $37,3,4425975,1,2.7,0.787,0.203,0.203,0.032,0,0,0,0,0,1,1,1,48,0.25,10$ $37,3,4415334,1,1.52,0.432,0.0432,0.203,0.0388,0,0,0,0,0,1,1,1,51,0.25,10$

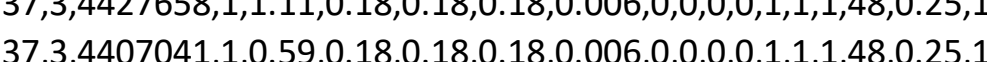
$37,3,4401354,1,0.38,0.18,0.18,0.18,0.006,0,0,0,0,1,1,1,48,0.25,10$ $37,3,4415382,0,12.73,0.584,0.381,0.8388,0.186,0,0,0,0,0,0,1,0,51,0.25,10$ $37,1,4441599,1,103,0.584,0.381,0.559,0.124,0,1,1,0,1,1,1,1,51,0.25,30$

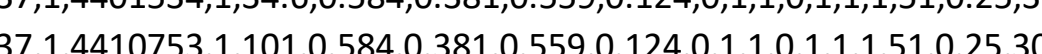
$37,1,4410667,1,50.2,0.584,0.381,0.559,0.124,0,1,1,0,1,1,1,51,0.25,30$ $37,1,4410565,0,127,1.981,0.381,0.381,0.288,0,1,1,0,1,1,1,1,80,0.25,150$ $37,1,4410561,0,26,2.21,0.305,0.335,0.0206,0,0,0,0,0,1,1,0,32,0.125,10$ $38,3,4367939,1,1010.432,0.432,0203,0.038,0,0,0,01,1,1,51,025,10$ $38,3,4388095,1,9.1,0.432,0.432,0.0203,0.038,0,0,0,0,0,1,1,151,0.25,10$ $38,1,4387962,1,34,1.143,0.762,0.533,0.464,0,1,0,0,1,1,1,1,51,0.25,30$ $38,1,4375817,1,150,1.143,0.762,0.0333,0.464,0,1,0,0,1,1,1,151,0.25,30$ $38,1,4373548,1,143,1.143,0.762,0.533,0.444,0,1,1,0,0,1,1,1,51,0.025,30$ $38,1.4361348,0,127.1 .143,1.143,1.321,1.726,0,1,0,0,1,1,1,51,0.2530$ $38,3,4371033,1,25.37,0.432,0.432,0.203,0.038,0,0,0,0,1,1,1,151,0.25,10$ $38,3,4388017,1,18.94,0.432,0.432,0.2033,0.038,0,0,0,0,0,1,1,1,51,0.25,10$

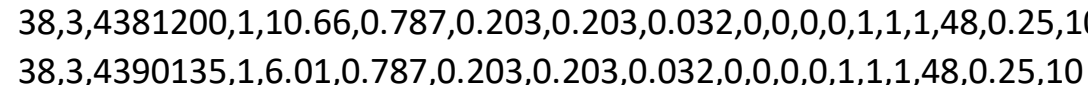

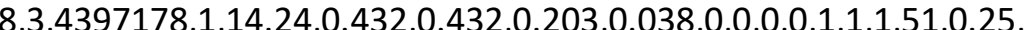
$38,3,4371031,1,13.66,0.432,0.432,0.203,0.038,0,0,0,0,0,1,1,1,51,0.25,10$ $38,3,4391775,1,2.29,0.18,0.18,0.18,0.006,0,0,0,0,0,1,1,1,48,0.25,10$ 3. $38,3,4370976,17.47,0.28,0.28,0.28,0.022,0,0,0,0,1,1,1,48,0.25,10$ $38,3,4388016,1,1.61,0.28,0.28,0.28,0.022,0,0,0,0,1,1,1,48,0.25,10$ $38,3,4390140,1,2.16,0.787,0.203,0.0203,0.032,0,0,0,0,0,1,1,1,48,0.25,10$

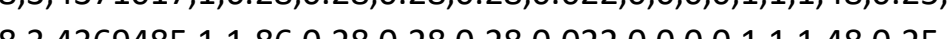

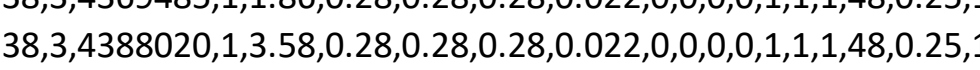
$38,3,4370971,1,2.21,0.28,0.28,0.28,0.022,0,0,0,0,1,1,1,48,0.25,10$ $38,3,4375911,1,3.43,0.787,0.203,0.203,0.032,0,0,0,0,0,1,1,1,48,0.25,10$ $38,1,4371007,1,115,0.061,0.61,0.66,0.246,0,1,0,0,0,1,1,1,1,51,0.25,30$ $38,3,4377555,1,16.94,0.533,0.533,0.432,0.123,0,0,0,0,0,1,1,48,0.25,10$ $38,3,4370991,1,14.8,0.533,0.533,0.432,0.123,0,0,0,0,0,1,1,48,0.25,10$ $38,1,4371043,1,120,0.61,0.061,0.66,0.246,0,0,1,0,0,1,1,1,1,51,0.25,35$

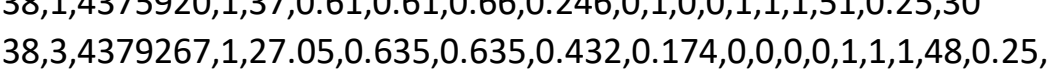

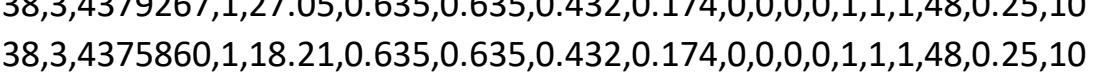
$38,1,4388080,1,58,1.143,0.762,0.914,0.796,0,1,0,0,1,1,1,1,0,0.25,30$ $38,1,4388015,0,090,1.143,0.762,1.041,0.907,0,1,0,0,1,1,1,1,51,0.25,30$ (3.

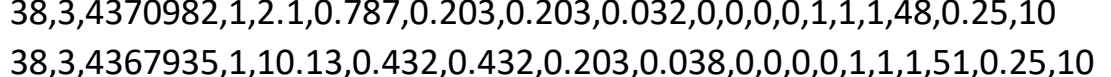

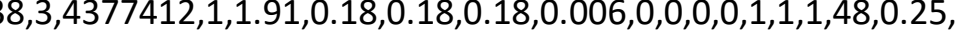
$38,3,43758333,1,1.75,0.18,0.18,0.018,0.006,0,0,0,0,0,1,1,1,4,48,0.25,10$

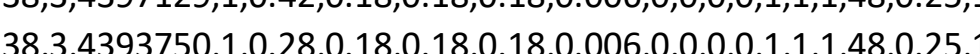

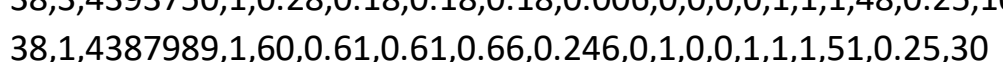
$38,1,4372617,1,40,0.584,0.381,0.559,0.124,0,1,1,0,1,1,1,51,0.25,30$ $38,3,4397247,1,4.72,0.432,0.432,0.432,0.081,0,0,0,0,1,1,1,48,0.25,10$ $38,3,4367927,1,10.1,0.432,0.432,0.023,0.0388,0,0,0,0,0,1,1,1,51,0.025,10$

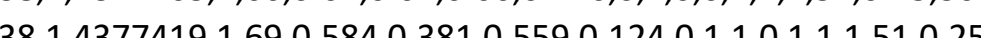
$38,1,4377431,1,59,0.584,0.381,0.559,0.124,0,1,1,0,1,1,1,51,0.25,30$ $38,3,4381120,1,16.86,0.533,0.533,0.432,0.123,0,0,0,0,0,1,1,1,48,0.25,10$ $38,3,4371014,1,9.2,0.533,0.533,0.432,0.123,0,0,0,0,0,1,1,1,1,48,0.25,10$ $38,1,4368090,0,1366,2.108,1.194,0.813,2.046,0,1,1,0,1,1,1.5,80,0.025,150$ $38,1,4397854,0,68,2.21,0.61,0.508,0.685,0,1,1,0,1,1,1,80,0.25,150$ 
$38,1,4395345,0,205.93,0.762,0.686,0.711,0.372,0,1,0,0,1,1,2,80,0.25,150$ $38,1,4368949,1,197,1.143,0.0762,0.533,0.464,0,1,0,0,0,1,1,1,1,51,0.25,30$ $38,1,4377468,1,185,1.143,0.7662,0.533,0.4644,0,1,0,0,1,1,1,1,51,0.25,30$ $38,1,4397156,0,155,1.143,1.143,0.33,0.431,0,1,0,0,1,1,1,1,8,0,0.25,150$ $38,1,4397153,0,05,1.143,1.143,0.33,0.431,0,1,0,0,1,1,1,80,0.25,150$ $38,1,4390165,0,118,1.143,1.143,1.346,1.758,0,1,0,0,1,1,1,51,0.25,30$ $38,1,4368947,1,131,1.143,0.762,0.533,0.464,0,1,0,0,1,1,1,51,0.25,30$ $38,1,4379286,1,193,1.143,0.762,0.533,0.464,0,1,0,0,1,1,1,1,151,0.25,30$ $38,1,4390177,0,09,1.143,1.143,1.092,1.427,0,1,0,0,1,1,1,51,0.25,30$ $38,1,4375909,1,58,1.143,0.762,0.533,0.464,0,1,0,0,1,1,1,51,0.25,30$ $38,1,4390168,1,84,1.143,1.143,0.9655,1.261,0,1,0,0,0,1,1,1,51,0.25,30$ $39,1,4401521,1,77,0.584,0.381,0.0559,0.124,0,1,1,0,1,1,1,1,0.25,30$ $39,1,4377542,1,30,0.584,0.381,0.559,0.124,0,1,1,0,1,1,1,51,0.25,30$ $39,1,4377554,1,39.4,0.584,0.381,0.559,0.124,0,1,1,1,1,1,1,51,0.25,30$ (3, $1,4399708,1,1,3,0.584,0.381,0.559,0.124,0,0,1,1,0,1,1,1,1,0.25,30$

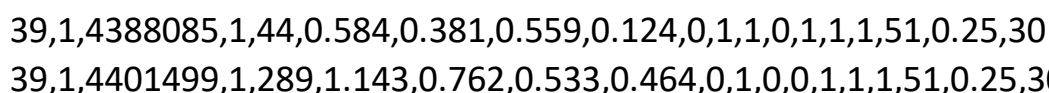
$39,1,4419516,1,97.98,1.143,0.762,0.559,0.487,0,0,0,0,1,1,1,510.25,30$ $39,1,4399907,1,72,1.143,0.762,0.533,0.0464,0,1,0,0,1,1,1,51,0.25,30$ $39,1,4415414,1,75,1.143,0.762,0.533,0.0464,0,1,0,0,0,1,1,1,51,0.25,30$ 3, 3,34405418,1,1,.83,1,1.06, $39,3,4399818,1252,0.28,0.28,0.28,0.022,0,0,0,0,1,1,48,0.25,10$ $39,3,4397273,1,9.11,0.533,0.533,0.432,0.123,0,0,0,0,1,1,1,48,0.25,10$ $39,3,4386770,1,2.46,0.28,0.28,0.28,0.022,0,0,0,0,0,1,1,1,48,0.25,10$ (3), $39,1,4375910,1,58.6,1,143,0.762,0.533,0.0644,0,1,0,0,1,1,51,0.25,30$ $39,1,4417544,1,40.2,1.143,0.762,0.533,0.464,0,1,0,0,1,1,1,51,0.25,30$ $39,3,4377574,1,30,0.635,0.635,0.432,0.174,0,0,0,0,0,1,1,1,48,0.25,10$ 年,

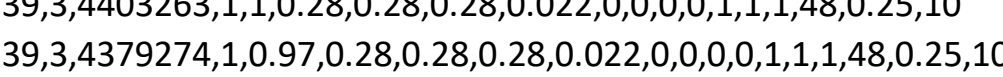
$39,3,4401403,1,0.9,0.28,0.28,0.28,0.022,0,0,0,0,1,1,1,48,0.25,10$ $39,3,4377518,1,16.97,0.432,0.432,0.203,0.0388,0,0,0,0,0,1,1,1,151,0.25,10$ (3, $3,3,401405,1,13.25,0.432,0.432,0.023,0.038,0,0,0,0,0,1,1,1,1,1,0.25,10$ $39,3,4375802,1,5.12,0.432,0.432,0.203,0.038,0,0,0,0,1,1,1,51,0.25,10$ $39,3,4388032,1,9.07,0.28,0.28,0.28,0.022,0,0,0,0,0,1,1,1,48,0.25,0$ $39,3,4388105,1,4,0.28,0.28,0.28,0.022,0,0,0,0,0,1,1,1,48,0.25,10$

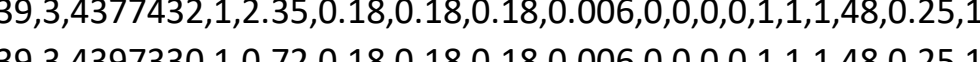
$39,3,4407204,1,0.38,0.18,0.18,0.18,0.006,0,0,0,0,1,1,48,0.25,10$ $39,3,4408790,1,0.36,0.18,0.18,0.18,0.006,0,0,0,0,1,1,1,1,48,0.25,10$ $39,1,4401387,1,45,1.143,0.762,0.0533,0.464,0,1,1,0,0,1,1,1,51,0.25,30$ (3.,

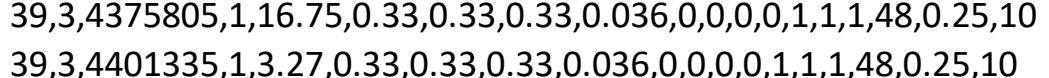
9 $3,3,4403365,1,5.5,0.33,0.33,0.33,0.036,0,0,0,0,0,1,1,1,48,0.25,10$ $39,3,43888888,1,2.13,0.33,0.333,0.33,0.036,0,0,0,0,0,1,1,1,148,0.25,10$ $39,3,4387927,1,12.2,2,066,0.66,0.66,0.287,0,0,0,0,1,1,1,48,0.25,10$ $39,1,4375828,0,100,1.143,1.143,0.33,0.0431,0,1,0,0,0,1,1,1,80,025,150$ $39,1,4415431,1,73,1.143,0.762,0.914,0.796,0,1,0,0,1,1,1,180,0.25,30$ $39,1,4404160,0,53,1.143,1.143,0.432,0.564,0,1,0,0,1,1,1,80,0.25,150$ $39,3,4407239,1,20.81,0.635,0.635,0.432,0.174,0,0,0,0,0,1,1,1,48,0.25,10$ $39,34015480,1959,035605590432200860,0,0,1,1,51,25,10$ $39,3,4403319,1,12.64,0.777,0.203,0.203,0.032,0,0,0,0,1,1,1,48,0.25,10$ $39,3,4407175,1,5.4,0.787,0.203,0.203,0.032,0,0,0,0,0,1,1,1,48,0.25,10$ $39,1,4401494,0,91,0.61,0.457,0.483,0.0135,0,1,0,0,1,1,1,1,80,0.25,150$

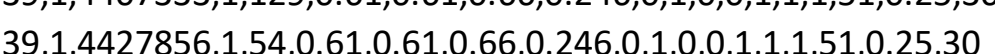
99, $1,4401393,1,112,0.61,0.61,0.66,0.246,0,1,0,0,1,1,1,51,0.25,30$ (9, $1,4422624,1,35,0.61,0.61,0.66,0.246,0,1,0,0,1,1,1,1,1,0.25,30$ $39,1,4419520,0,01039.18,1.549,1.143,1.067,1.889,0,1,1,0,1,1,2,2,80,0.25,150$ $39,3401407,1,50.4320 .4320 .4320 .081000,0,1,1,1,48,025,10$ $39,3,4387480,1,2.02,0.28,0.28,0.28,0.022,0,0,0,0,1,1,1,48,0.25,10$

$39,3,4405081,1,1.36,0.28,0.28,0.28,0.022,0,0,0,0,0,1,1,1,1,4,0.025,10$ $39,3,4379262,1,0.29,0.18,0.18,0.018,0.006,0,0,0,0,1,1,1,1,48,0.25,10$ $40,1,4429307,7,1,73,1.143,1,1.143,0.965,1.261,0,0,1,0,0,1,1,1,51,0.25,30$ $40,1,4429295,0,124,1.143,1.143,1.321,1.726,0,1,0,0,1,1,151,0.25,30$ $40,1,4420478,1,77,1.143,0.762,0.914,0.796,0,1,0,0,0,1,1,1,80,0.25,30$ $40,1,4429472,1,32,1,143,0.762,0.914,0.796,0,1,0,0,1,1,1,1,80,0.025,30$

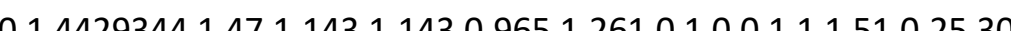
$40,1,4429463,0,116,1.143,1.143,1.321,1.726,0,1,0,0,1,1,1,51,0.25,30$ $40,1,4429528,0,138,1.016,1.016,2.007,2.072,0,1,0,0,1,1,1,80,0.25,150$ $40,1,4429555,0,138,1.016,1.016,2.007,2.072,0,1,0,0,0,1,1,1,80,0.25,150$ $40,1442952401381,24510162007,2539,0,1,0,1,1,1,1,0,025,150$ $40,1,4429609,0,204,1.27,0.813,0.533,0.55,0,1,0,1,1,1,1,80,0.25,150$ $40,1,4429697,0,53,1.143,0.762,1.143,0.996,0,1,0,0,1,1,1,151,0.25,30$ $40,1,4428862,0,0314,1.143,1.143,0.381,0.498,0,1,0,0,0,1,1,1,80,0.25,150$

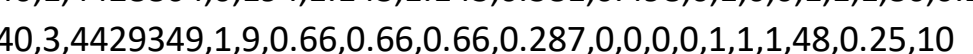
$40,3,4429130,1,2.711,0.33,0.33,0.33,0.036,0,0,0,0,1,1,1,48,0.25,10$ $40,3,4436655,1,0.26,0.18,0.18,0.18,0.006,0,0,0,0,1,1,1,48,0.25,10$ $40,1,4429230,0,132,1.143,1.143,0.27,0.364,0,1,0,0,0,1,1,1,80,0.25,150$ $40,1,1429226,0,13,11.143,1.143,1.372,1.792,0,1,0,0,1,1,1,1,51,0.25,30$ (40,1,4428859,0,112,0.61,0.61, $0.66,0.246,0,1,0,0,1,1,1,51,0.25,30$ $40,1,4428971,0,281,2.261,0.33,0.0406,0.303,0,1,0,1,1,1,1,1,180,0.25,150$ $40,1,4229560,0,115,1.143,1.143,1.448,1.892,0,1,0,0,1,1,1,51,0.25,30$ $40,1,4292201110,1433,076209140,096,0,1,0,1,1,1,1,0.25,30$ $40,1,4429210,179,1143,0.762,0.914,0.796,0,1,0,0,1,1,180,0.25,30$ $40,1,4429221,1,106,1.143,0.762,0.914,0.796,0,1,0,0,1,1,1,80,0.25,30$ $40,1,4429553,0,06,1.067,1.067,1.321,1.504,0,1,0,0,1,1,1,1,80,0.25,150$ (1)

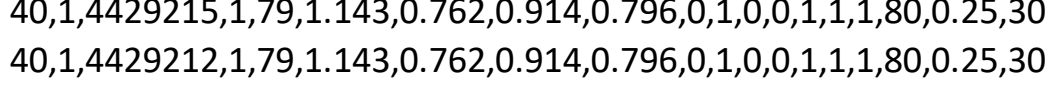
$41,1,4407295,0,451.5,2.286,1.143,2.21,5.775,0,1,1,0,1,1,1,80,0.25,150$ $41,1,4407288,0,451,2.2286,1.143,2.221,5.775,0,1,1,1,0,1,1,1,1,80,0.25,150$ $41,1,407257,1,164,1133,0762,0533,0464,0,1,0,1,1,1,51,0.25,30$ $41,1,4407144,1,77,8,1433,0.762,0.533,0.464,0,1,0,0,1,1,1,01,0,25,30$ $41,1,4407332,1,146,1.143,0.762,0.965,0.84,0,1,0,0,1,1,1,51,0.25,30$ $41,1,4407329,0,089,1.143,0.0762,1.245,1.084,0,1,0,0,1,1,1,1,51,0.25,30$ (4, $41,1,44072929,0,434.5,2.2286,1.143,2.2 .159,5.6441,0,1,1,1,1,1,80,025,150$ $41,1,4407298,0,388,2.286,1.143,2.208,5.508,0,1,1,0,1,1,1,80,0.25,150$ $41,1,4407301,0,411,2.286,1.143,2.2032,5.309,0,1,1,0,1,1,1,1,80,0.25,150$

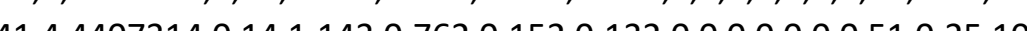

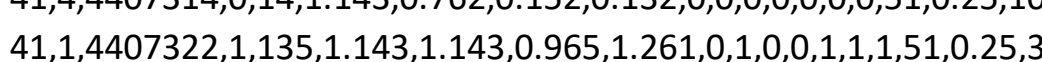
$41,1,4407306,0,158,1.143,1.143,1.219,1.593,0,1,1,0,0,1,1,1,1,1,0.25,30$ $41,1,4407318,0,165,1.143,1.143,1.549,2.2024,0,1,0,0,1,1,1,51,0.25,30$ 4.

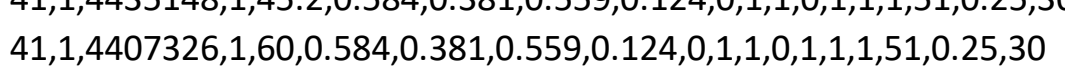
$41,1,4406769,0,235,1.981,1.092,0.432,0.935,0,1,2,1,1,1,1,5,80,0.25,150$ $41,1,4429779,0,44,0.61,0.61,0.737,0.274,0,1,0,0,1,1,1,51,0.25,30$ (1, $1,3452573,1,8.14,0.533,0.533,0.432,0.123,0,0,0,0,1,1,1,1,48,0.25,10$ $41,3,423977,1,0.47,0.18,0.18,0.18,0.006,0,0,0,1,1,1,48,0.25,10$ $41,3,4452498,1,12.65,0.432,0.432,0.203,0.038,0,0,0,0,1,1,51,0.25,10$ $41,1,4435240,0,228,0.889,0.559,0.457,0.227,0,1,1,0,1,1,1.5,580,0.25,150$ $41,1,4435161,0,257,0.762,0.0762,0.686,0.0398,0,1,0,0,1,1,1,1,58,0,0.25,150$ $42,1,4446281,0,121,1.88,0.33,0.33,0.205,0,1,1,1,0,1,1,1,1,80,0.25,150$ $42,1,4463271,0,67,1.143,0.762,0.33,0.287,0,1,0,0,1,1,1,80,0.25,150$ 
$42,1,4492907,0,104,1,143,1,143,0.33,0.431,0,0,1,0,0,1,1,1,1,80,0.25,150$ $42,1,4493196,1,40.1,0.584,0.381,0.559,0.124,0,0,1,1,0,1,1,1,51,0.025,30$ $42,1,4499951,1,30,0.584,0.0381,0.559,0.124,0,0,1,1,0,1,1,1,1,51,0.25,30$ $42,1,4492908,0,104,1.143,1.143,0.33,0.431,0,1,1,0,0,1,1,1,1,80,0.25,150$

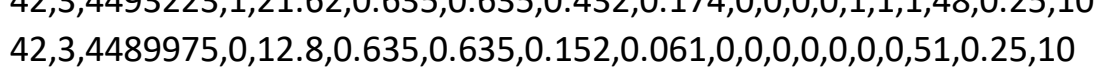
$42,3,4493315,1,125.04,0.432,0.432,0.203,0.038,0,0,0,0,0,1,1,1,51,0.25,10$ $42,3,4467973,1,9.8,0.432,0.432,0.203,0.038,0,0,0,0,0,1,1,1,51,0.25,10$ $42,344900341,1560.28028028002200,0,1,1,1,5,25,150$ $42,3,4495247,1,0.99,0.28,0.28,0.28,0.022,0,0,0,0,0,1,1,1,48,0.25,10$ $42,3,4489868,1,0.68,0.18,0.18,0.18,0.006,0,0,0,0,1,1,1,48,0.25,10$ $42,1,4487916,1,197,1.143,0.762,0.533,0.464,0,1,0,0,0,1,1,1,51,0.25,30$ $42,3,449327,1,14.21,0.432,0.42,0.432,0.081,0,0,0,1,1,1,4,4,0.25,10$ $42,1,4467879,0,113,1.93,0.178,0.0279,0.096,0,0,1,0,1,1,1,80,0.25,150$ $42,1,4465306,1,129,0.61,0.61,0.66,0.246,0,1,0,0,1,1,1,51,0.25,30$ $42,1,4993297,1,124,1.143,0.762,0.53,0.0464,0,1,0,0,1,1,1,51,0.25,30$ $42,1,4465275,1,115,0.61,0.061,0.66,0.246,0,1,0,0,0,1,1,1,51,0.25,30$
$42,1,4493307,1,98,0.61,0.61,0.66,0.246,0,1,0,1,1,1,51,0.25,30$ $42,1,4463215,0,101,193,0.533,0.584,0.001,0,1,1,0,1,0.5,80,0.25,150$ $42,1,4990048,1,42,1.143,0.762,0.533,0.464,0,1,0,0,1,1,1,1,151,0.025,30$ $42,1,4464674,0,1115,1.803,0.055,0.711,0.717,0,1,1,1,0,1,1,0.5,80,0,0.25,150$ $42,1,4493260,1,86.6,6,0.61,0.061,0.66,0.246,0,1,0,0,0,1,1,1,51,0.25,30$ $42,1,4493168,0,479,5.613,0.381,0.33,0.706,0,1,1,0,1,1,1,0,025,150$ $42,1,4447899,0,120,2.845,0.559,0.66,1.05,0,1,1,0,0,1,1,1,80,0.25,150$ $42,1,4493093,0,56.5,2.946,0.305,0.457,0.411,0,1,1,0,0,1,0,80,0.25,150$

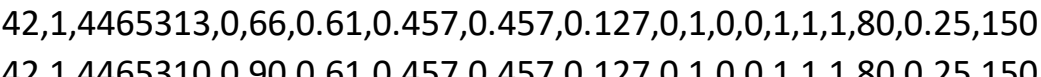
$42,1,4489769,0.910 .61,0.457,0.457,0.127,0,1,2,0,1,1,1,80,0.25,150$ $42,1,4493311,0,95,1.016,0.279,0.406,0.115,0,1,2,0,1,1,1,80,0.25,150$ $43,1,4469901,0,89,0.61,0.457,0.457,0.127,0,1,1,0,1,1,1,80,0.25,150$ $43,1,466915,0,78,0.61,0447,0457,0.127,0,1,1,0,1,1,1,0,0.25,150$ $43,1,4663214,0,099,1.346,0.178,0.254,0.061,0,1,0,0,1,1,1,80,0.25,150$ $43,1,4461450,1,133,1.143,0.762,0.533,0.464,0,1,0,0,1,1,1,1,51,0.25,30$ $43,1,4478515,1,54,1.143,0.762,0.914,0.796,0,1,0,0,1,1,1,1,80,0.25,30$ $43,1,448484,1,13,1.143,0.762,0.914,0.796,0,1,0,0,1,1,1,180,0.25,30$ $43,1,4478473,01171,143,1143,1321,1726,0,1,0,0,1,1,51,025,30$ $43,1,4478425,0,117,1.143,1.143,1.321,1.726,0,1,0,0,1,1,1,51,0.25,30$ $43,1,4478442,0,117,1.143,1.143,1.321,1.72726,0,1,0,0,1,1,1,1,51,0.25,30$ $43,1,4464724,0,25,2.21,0.381,0.305,0.257,0,0,0,0,1,0,0,51,0.25,10$

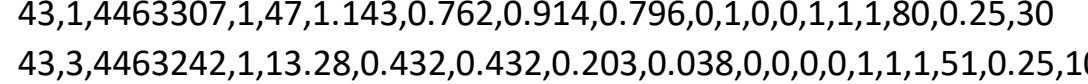
$43,3,4482454,1,1.55,0.28,0.28,0.28,0.022,0,0,0,0,1,1,1,48,0.25,10$ $43,3,44772540,1,3,4,0,0.787,0.2033,0.203,0.03232,0,0,0,0,1,1,1,1,48,0.25,10$ $43,3,477257,1,15.3,0.33,0.33,0.33,0.063,0,0,0,0,1,1,1,48,025,10$

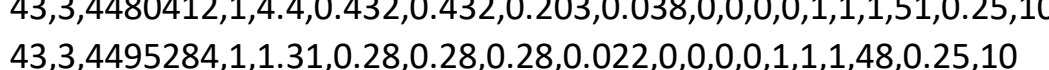
$43,3,4501037,1,1.25,0.28,0.28,0.28,0.022,0,0,0,0,111,48,0.25,10$ $43,3,4499026,1,0.98,0.28,0.28,0.028,0.022,0,0,0,0,0,1,1,1,48,0.025,10$ 43,3,4501151,1,5.7,0.28,0.28,0.28,0.022,0,0,0,0,1,1,1,48,0.25,10

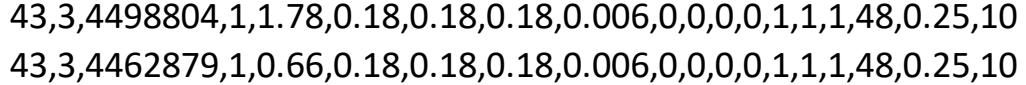
$43,1,4478488,0,135,1.143,1.143,1.397,1.825,0,1,0,0,1,1,1,51,0.25,30$ $43,3,4499065,1,12.25,1.016,0.28,0.28,0.08,0,0,0,0,0,1,1,1,51,0.25,10$ $43,3,4499061,1,12.13,1.016,0.28,0.28,0.08,0,0,0,0,0,1,1,1,51,0.25,10$

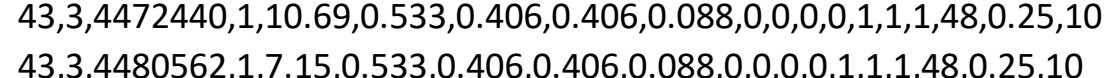
$43,3,3469837,1,2.64,1.016,0.28,0.28,0.08,0,0,0,0,1,1,1,51,0.25,10$ $43,3,4478420,1,4.97,0.432,0.432,0.432,0.081,0,0,0,0,0,1,1,48,0,0.25,10$ $43,3,4499038,1,0.45,0.18,0.18,0.18,0.006,0,0,0,0,1,1,1,48,0.25,10$

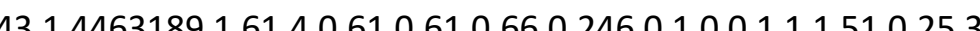
$43,1,4463218,1,97,0.584,0.381,0.559,0.124,0,1,1,0,1,1,1,51,0.25,30$ $43,1,4469821,0,59,2.845,0.356,0.457,0.463,0,1,1,0,0,1,1,80,0.25,150$ $43,1,4478518,0,127,1.143,1.143,1.524,1.991,0,1,0,0,1,1,1,1,0,0.25,30$ $44,14453731,0116,1143,1143,13461.758,0,1,0,1,1,1,51,025,30$ $44,1,4448841,1,123,1,143,1,43,0965,1261,01,0,1,1,1,51,025,30$ $44,1,4453754,0,116,1.143,1.143,1.346,1.758,0,1,0,0,1,1,1,51,0.25,30$ $44,1,4448807,1,187,1.143,1.143,0.965,1.261,0,1,0,0,0,1,1,1,51,0.25,30$ $44,1,4448844,178,1,143,1,143,1,0655,1261,0,1,0,0,1,1,51,0.25,30$ $44,1,4448789,0,117,1.143,1.143,1.321,1.726,0,1,0,0,1,1,1,51,0.25,30$ $44,1,4446696,1,44.6,0.584,0.381,0.965,0.215,0,1,1,0,1,1,1,51,0.25,30$ $44,1,4446972,1,100,0.584,0.381,0.559,0.124,0,1,1,0,1,1,1,51,0.025,30$ $44,3437535,1240.432,0432,02030.038,0,0,0,1,1,51,025,10$ $44,1,4446968,1,53,0.584,0.381,0.559,0.124,0,1,1,0,1,1,1,51,0.25,30$ $44,1,4453756,1,50,1.143,0.762,0.965,0.84,0,0,1,0,0,1,1,1,51,0.25,30$ $44,1,4441170,1,1111,1.143,0.762,0.914,0.0 .996,0,1,0,0,1,1,1,1,80,0.25,30$

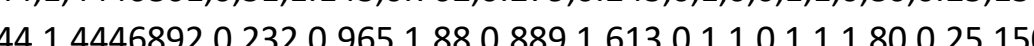
$44,1,4446894,0,232,0.965,1.88,0.889,1.613,0,1,1,0,1,1,1,80,0.25,150$ $44,1,4463267,1,84,1.143,0.762,0.533,0.464,0,1,0,0,1,1,1,151,0.25,30$ $44,1,4453526,0,0,129,1.143,1.143,0.33,0.441,0,1,0,0,1,1,1,80,0.25,150$

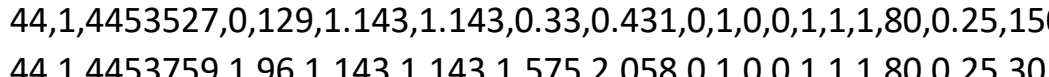
$44,1,4446934,0,78,0.61,0.457,0.457,0.127,0,1,0,0,1,1,1,00,025,150$ $44,3,4446815,1,1.46,0.28,0.28,0.28,0.022,0,0,0,0,1,1,1,48,0.25,10$ $44,3,4448726,1,5.73,0.28,0.028,0.28,0.022,0,0,0,0,0,1,1,1,48,0.25,10$ $44,3,4446883,1,3.33,0.28,0.28,0.28,0.022,0,0,0,0,0,1,1,1,1,48,0.25,10$

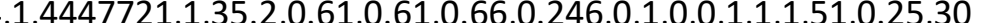
$44,1,4446949,0,71.8,0.61,0.61,0.711,0.265,0,1,0,0,1,1,1,51,0.25,30$ $44,1,4458866,1,197,1.143,0.762,0.533,0.464,0,0,0,0,1,1,1,51,0.025,30$

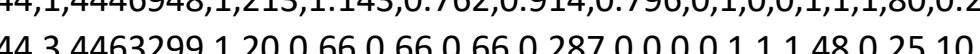
$44,1,4446978,1,90,0.61,0.61,0.66,0.246,0,0,0,1,1,1,51,025,30$ $44,3,4429695,1,23.5,0.533,0.533,0.432,0.123,0,0,0,0,1,1,1,48,0.25,10$

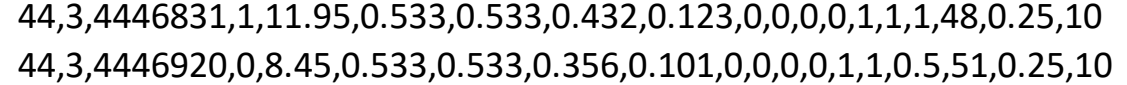
(4) $44,1.4461369,0,62,1.626,0.178,0.203,0.059,0,1,0,0,1,1,80,0.25,150$ $44,3,4446712,10.68,0.18,0.18,0.18,0.006,0,0,0,0,0,1,1,48,0.25,10$ $44,3,4446710,1,0.43,0.18,0.18,0.18,0.006,0,0,0,0,0,1,1,1,48,0.25,10$

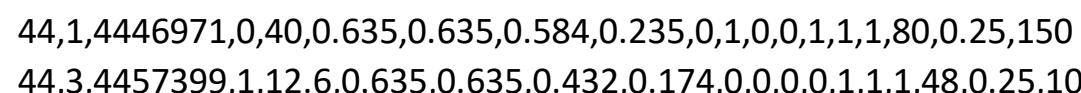
$45,1,4477959,1,49,1.143,0.762,0.965,0,84,0,0,0,1,1,1,1,0,05,30$ $45,1,4477950,0,173,1.143,0.762,1.194,1.04,0,1,0,0,1,1,1,51,0.25,30$

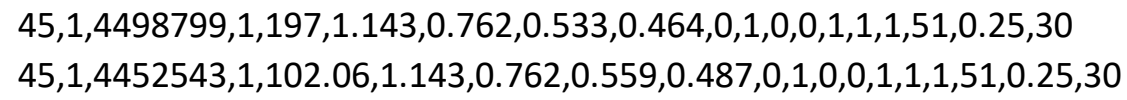
$45,1,4452543,1,102.06,1.143,0.762,0.559,0.487,0,1,0,0,1,1,1,1,51,0.25,30$

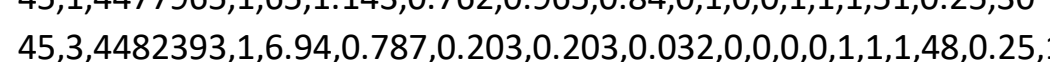
$45,1,4474365,1,115,0.61,0.61,0.66,0.246,0,1,0,0,1,1,1,51,0.25,30$ $45,1,4499020,1,54.8,0.61,0.61,0.66,0.244,0,0,0,0,1,1,1,51,0.25,30$

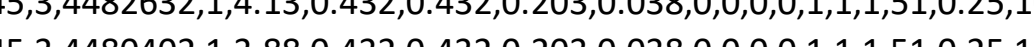
$45,3,4480402,1,3.88,0.432,0.432,0.0203,0.038,0,0,0,0,0,1,1,1,51,0.025,10$

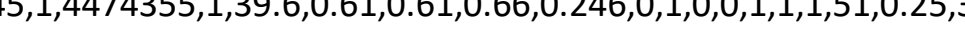
$45,1,4482489,1,38.6,6,0.61,0.61,0.066,0.246,0,1,0,0,1,1,1,1,51,0.25,30$

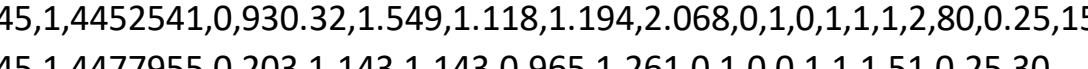

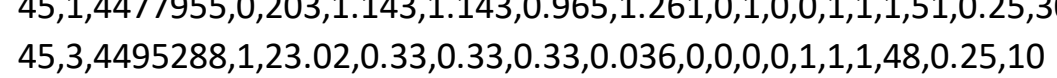
$45,3,4446940,1,5.92,0.33,0.33,0.33,0.036,0,0,0,0,1,1,1,148,0.25,10$ $45,1,4472571,0,80,2.718,0.457,0.533,0.662,0,1,2,2,0,0,1,0.5,80,0,0.25,150$ 45, $45,34990291,1,340.2802280280022200,0,0,1,1,48025,10$ $45,3,4465208,1,1,43,0.28,0.28,0.28,0.022,0,0,0,0,0,1,1,1,48,0,25,10$ $45,3,4450236,1,1,37,0.28,0.28,0.28,0.0222,0,0,0,0,0,1,1,1,4,48,0.25,10$

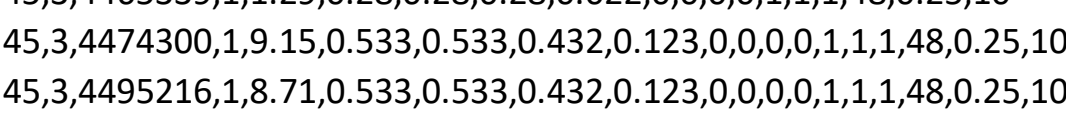



$45,3,4437476,1,15.09,0.432,0.432,0.203,0.038,0,0,0,0,1,1,1,51,0.25,10$ $45,3,4448695,1,1,84,0.432,0.432,0.203,0.038,0,0,0,0,0,1,1,1,51,0.25,10$

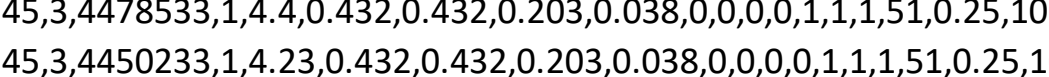
$45,3,4448581,1,5.15,0.18,0.18,0.18,0.006,0,0,0,0,1,1,1,48,0.25 .10$ $45,3,4446951,1,4.86,0.18,0.18,0.18,0.006,0,0,0,0,1,1,1,48,0.25,10$ $45,3,44632282,1,2.08,0.18,0.18,0.18,0.006,0,0,0,0,1,1,1,1,48,0.25,10$

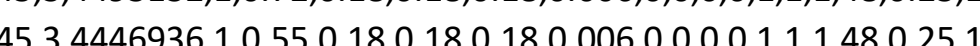
$45,3,4446957,1,8.37,0.33,0.33,0.33,0.036,0,0,0,0,1,1,1,48,0.25,10$ $45,3,4446722,1,0.44,0.18,0.18,0.18,0.006,0,0,0,0,1,1,1,148,0.25,10$ $45,3,44487723,1,0.233,0.18,0.18,0.18,0.006,0,0,0,0,1,1,1,1,48,0.25,10$ $45,14499032,1,196,0.584,0.381 .05590 .0124,0,1,1,0,1,1,510,2530$ $45,1,4495295,1,131.2,0.584,0.381,0.559,0.0124,0,1,1,0,1,1,1,51,0.25,30$ $45,1,4495340,1,7,0,0.584,0.381,0.559,0.124,0,1,1,0,1,1,1,51,0.25,30$ $45,1,4437553,0,07,0.64,0.0457,0.457,0.127,0,1,0,0,0,1,1,1,1,8,0.025,150$

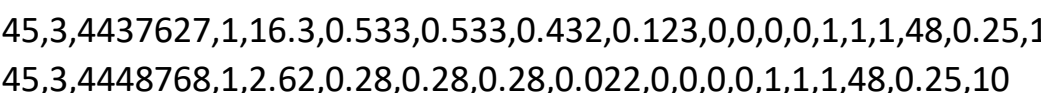
$45,1,4495309,1,51,0.584,0.381,0.559,0.124,0,1,1,0,1,1,1,51,0.25,30$ $45,1,4446983,0,072,1.016,0.0279,0.305,0.086,0,1,1,0,1,1,1,0.5,80,0.25,150$ $45,3,4446952,1,13,69,0.28,0.28,0.28,0.022,0,0,0,0,0,1,1,1,48,0.25,10$ $45,1,4448825,1,30,0.584,0.381,0.559,0.124,0,0,1,1,0,1,1,1,51,0.25,30$ $45,3,4477810,1,8.31,0.432,0.432,0.432,0.081,0,0,0,0,1,1,48,0.25,10$ $45,1,4477808,0,38,0.584,0.381,0.61,0.136,0,0,1,0,0,1,1,1,51,0.25,30$ $45,3,4503521,1,1.66,0.28,0.28,0.28,0.022,0,0,0,0,0,1,1,1,4,4,0.25,10$ (5)

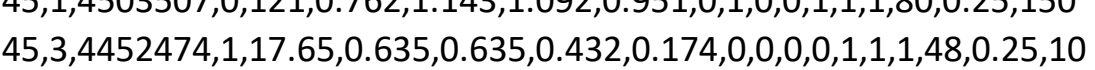
$45,3,4498999,1,13.55,0.635,0.635,0.432,0.174,0,0,0,0,0,1,1,1,48,0.25,10$ $45,1,4448821,0,78,1.1433,1.143,0.787,1.028,0,0,1,0,0,1,1,1,1,80,0.25,150$ $45,1,47743550,1,74,1.143,0.762,0.914,0.996,0,1,0,0,1,1,1,1,80,0.25,30$ $45,1,4477958,0,122,1.143,1.143,1.575,2.058,0,1,0,0,1,1,51,0.25,30$ $45,3,4498974,1,11,0.635,0.635,0.432,0.174,0,0,0,0,0,1,1,1,48,0.25,10$ $45,3,482539,0,14.17,0.635,0.035,0.127,0.011,0,0,0,0,1,1,1,1,51,0.25,10$ $46,1,4530414,0.489 .5,1.143,0.94,0.381,0.409,0,12,0,1,1,15,80.025,150$ $46,1,4530411,0,483,1.143,0.94,0.381,0.409,0,1,2,0,1,1,1,1.5,80,0.25,150$ $46,1,4526513,0,1133,1.143,1.143,1.321,1.726,0,1,0,0,0,1,1,1,51,0.25,30$ $46,1,4549814,0,17,1.193,0.864,0.381,0.725,0,1,2,0,1,1,1,1,5,80,0.25,150$ $46,3,4557603,1223,0.787,0.20302030 .03200,0,0,1,1,48,025,10$ $46,3,4557795,1,3.02,0.432,0.432,0.203,0.038,0,0,0,0,1,1,1,51,0.25,10$ $46,3,4569406,1,0.56,0.18,0.18,0.18,0.0006,0,0,0,0,1,1,1,1,48,0.25,10$

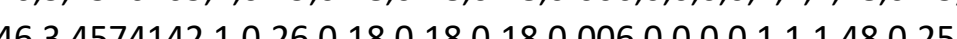
$46,3,4563369,1,0.23,0.18,0.18,0.18,0.0006,0,0,0,0,0,1,1,1,48,0.25,10$ $46,1,4550227,1,84,1.143,1.143,0.965,1.261,0,1,0,0,1,1,1,51,0.25,30$ $46,1,4526618,1,77,1.143,1.143,0.965,1.261,0,1,0,0,1,1,1,51,0,0.25,30$ $46,1,4531233,0,300,1.118,1.067,0.406,0.0484,0,1,0,0,1,1,1,1,80,0.25,150$ $46,1,45501621,78,1143,07620.0650,0840,0,0,1,1,51,025,30$ $46,3,4563458,1,13.56,1.016,0.28,0.28,0.08,0,0,0,0,0,1,1,1,51,0.25,10$ $46,3,4526414,1,4.9,0.28,0.28,0.28,0.022,0,0,0,0,0,1,1,1,48,0.25,10$ $46,3,4526538,1,2,15,0.28,0.28,0.28,0.022,0,0,0,0,0,1,1,1,1,48,0.25,10$ $463,4530373,1498028028028002200,0,0,1,1,48,025,10$ $46,3,4533568,1,2.56,0.28,0.28,0.28,0.022,0,0,0,0,1,1,1,48,0.25,10$ $46,3,4563492,1,1,78,0.28,0.28,0.28,0.022,0,0,0,0,1,1,1,1,48,0.25,10$ $46,3,4551565,1,1.14,0.28,0.28,0.28,0.0222,0,0,0,0,0,1,1,1,48,0.25,10$

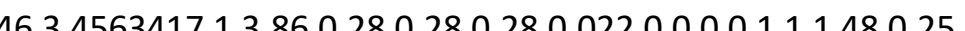
$46,3,4550129,1,2.144,0.28,0.28,0.28,0.022,0,0,0,0,1,1,1,48,0.25,10$ $46,3,45635518,1,1,1.38,0.28,0.028,0.028,0.022,2,0,0,0,0,0,1,1,1,48,0.25,10$ $46,3,4563605,1,6.82,0.33,0.33,0.33,0.036,0,0,0,0,1,1,1,1,48,0.25,10$

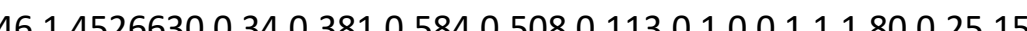
$46,1,4526622,0,21,0.584,0.381,0.991,0.221,0,1,0,0,1,1,1,51,0.25,30$ $46,1,4526676,1,169,0.584,0.381,0.559,0.124,0,1,1,1,0,1,1,1,51,0.25,30$ $46,1,4563872,1,53,0.584,0.381,0.559,0.124,0,1,1,1,0,1,1,1,51,0.25,30$ $46,1,454325,1,1,83,1.143,0.762,0.914,0.796,0,1,0,0,1,1,1,1,80,0.25,30$ $46,1,4529504,1,198,1.143,0.762,0.533,0.464,0,1,0,0,1,1,51,025,30$ $46,1,4567805,1,189,1.143,0.762,0.533,0.464,0,1,0,0,1,1,1,51,0.25,30$ $46,1,4531675,1,34,1.143,0.0762,0.533,0.464,0,0,1,0,0,1,1,1,51,0.25,30$

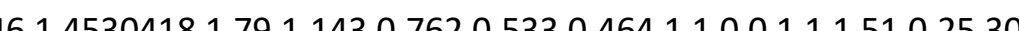
$46,1,4530409,1,161.6,1.143,0.762,0.533,0.464,0,1,1,0,0,1,1,1,51,0.25,30$ $46,1,4550269,1,55.8,1.143,0.762,0.533,0.464,0,1,0,0,1,1,1,51,0.25,30$ $46,1,4526558,1,49,1.143,0.762,0.533,0.464,0,1,0,0,1,1,1,151,0.25,30$ $46,1453042201,1160.58403810559012401,1,1,1,1,01,0.25,30$ $46,1,4532052,1,52,0.584,0.381,0.559,0.124,0,1,1,0,1,1,1,51,0.25,30$ $46,3,4557817,1,14.26,0.432,0.432,0.432,0.081,0,0,0,0,1,1,1,1,48,0.25,10$ $46,3,4526426,1,11.17,0.533,0.406,0.406,0.088,0,0,0,0,0,1,1,1,4,48,0.25$,

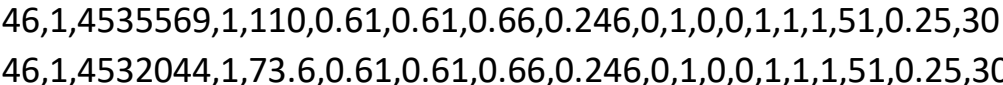
$46,3,4526323,1,10,0.533,0.533,0.432,0.123,0,0,0,0,0,1,1,48,0.25,10$ $46,3,4526555,1,8.43,0.533,0.533,0.432,0.123,0,0,0,0,1,1,1,48,0.25,10$ $46,1,4563706,1,30.8,0.584,0.381,0.965,0.215,0,0,1,0,0,1,1,1,5,1,0.25,30$

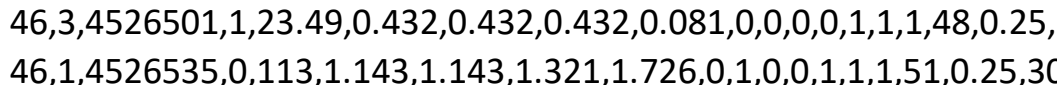
$46,14550130,1,100,1143,1143,0965,12610,0,0,1,1,51,01,02530$ $46,1,4550207,1,93,1.143,1.143,0.965,1.261,0,0,1,0,0,1,1,1,51,0.25,30$ $46,1,4526619,1,85,1.143,1.143,0.965,1.261,0,1,0,0,0,1,1,1,151,0.25,30$

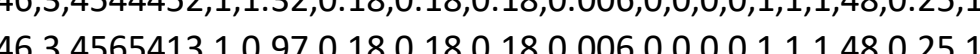
$46,3,4526298,1,0.81,0.18,0.18,0.18,0.006,0,0,0,0,1,1,148,0.25,10$ $47,1,4446321,0,120,1.88,0.33,0.33,0.205,0,1,1,0,1,1,1,80,0.25,150$ $47,1,4500971,0,112.5,1.88,0.381,0.381,0.273,0,1,2,2,0,1,1,1,80,0.25,150$ $47,3,426419,14,030202828,0281,0.028,0,1,0,0,1,1,1,1,0.025,30$ $47,1,4526170,0,115,1.143,1.143,0.33,0.431,0,1,0,0,1,1,80,0.25,150$ $47,1,4526171,0,115,1.143,1.143,0.33,0.431,0,1,0,0,1,1,1,80,0.25,150$ $47,1,4526172,0,104,1.143,1.143,0.33,0.431,0,0,1,0,0,1,1,1,180,0.25,150$ $47,1,4566755,172,1143,1.762,09140.07960,0,0,0,1,1,1,180,0.25,150$ $47,1,4526678,0,59,1041,0.305,0.406,0.129,0,1,0,1,1,0,580,025,150$ $47,1,4526666,1,130,0.584,0.381,0.559,0.124,0,1,1,0,1,1,1,51,0.25,30$ $47,3,4526539,1,19.02,0.33,0.33,0.33,0.036,0,0,0,0,0,1,1,1,48,0.25,10$

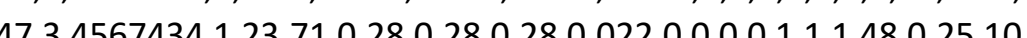
$47,3,4563544,1,13.26,0.28,0.28,0.28,0.022,0,0,0,0,1,1,48,0.25,10$ $47,1,4526690,1,49,0.584,0.381,0.559,0.124,0,1,1,0,1,1,1,51,0.25,30$ $47,1,4526626,1,50,0.584,0.381,0.559,0.124,0,0,1,1,0,1,1,1,51,0.025,30$

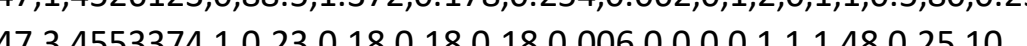

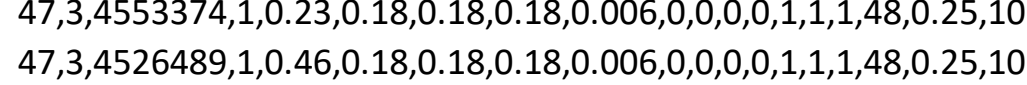
$47,3,4559874,1,8.47,0.432,0.432,0.432,0.081,0,0,0,0,1,1,1,48,0.25,100$ $47,1,4401428,0,215,3.399,0.737,0.711,1.624,0,1,1,0,0,1,1,1,80,0.25,150$

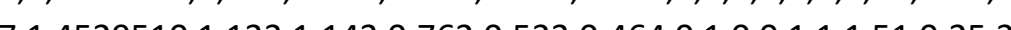

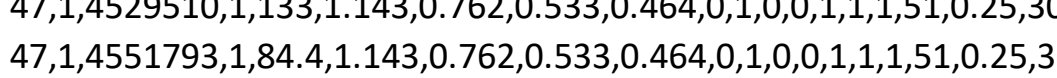

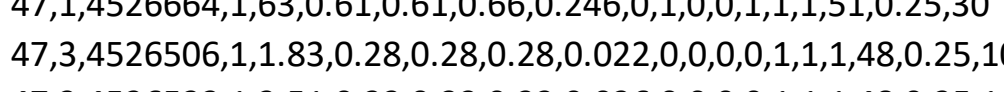

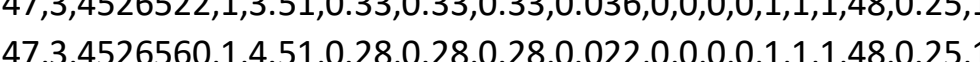
$47,3,4526509,1,1.85,0.28,0.28,0.28,0.022,000,0,1,1,48,0.25,10$ 77,3,4555298,1,0.5,0.18,0.18,0.18,0.006, $0,0,0,0,1,1,1,48,0.25,10$ $47,1,4529509,1,133,1.143,0.0762,0.0333,0.464,0,1,0,0,1,1,1,51,0.25,30$ $47,1,4526658,1,1,1,1.143,0.762,0.533,0.464,0,1,0,0,0,1,1,1,1,51,0.25,30$

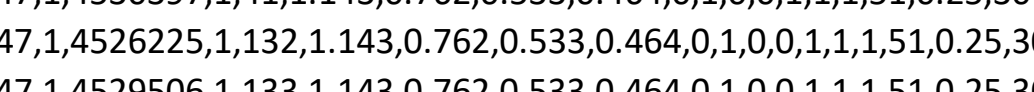

$47,1,4529506,1,133,1.143,0.762,0.533,0.464,0,1,0,0,1,1,1,51,0.25,30$ 
$47,1,4526127,0,1248,1.473,1.372,1.499,3.029,0,1,2,0,1,1,1,1.5,80,0.25,150$ $47,3,4526432,1,5,59,0.432,0.432,0.0203,0.038,0,0,0,0,0,1,1,1,51,0,0.25,10$ $47,1,4526226,1,196,1.1433,0.762,0.533,0.0464,0,1,0,0,1,1,1,1,51,0.25,30$ $47,1,4546583,1,72,1,143,0.0762,0.914,0.796,0,1,0,0,1,1,1,1,80,0.25,30$

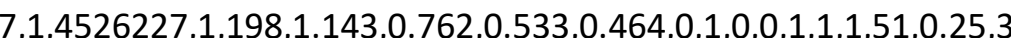
$47,1,4534601,1,70,1.143,0.762,0.914,0.796,0,1,0,0,1,1,1,80,0.25,30$ $47,1,4526562,1,27.6,0.584,0.381,0.559,0.124,0,0,1,1,0,1,1,1,51,0.25,30$ $47,3,4526679,1,169,0.280 .28,028,0.022,0,0,0,0,1,1,48,025,10$ $47,3,4559633,1,6.58,0.33,0.033,0.33,0.036,0,0,0,0,0,1,1,1,48,0.25,10$ $47,3,4526620,1,22.41,0.432,0.432,0.432,0.081,0,0,0,0,1,1,1,48,0.25,10$ $47,3,4526480,1,20.37,0.432,0.432,0.432,0.081,0,0,0,0,0,1,1,1,48,0.25,10$

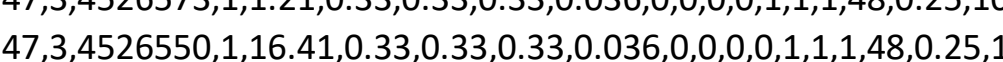
$47,1,4526665,1,107,0.61,0.61,0.66,0.246,0,1,0,0,1,1,1,51,0.25,30$ $47,1,4526518,1,28.06,0.61,0.61,0.66,0.246,0,1,0,0,1,1,1,51,0.25,30$ $47,1,4529524,0,147,1.219,0.813,0.0356,0.353,0,0,1,2,0,1,1,1,1.5,80,0.25,150$ $47,1,4563194,0,852,1.143,0.965,0.61,0.67,0,0,1,2,0,1,1,1,1,5,80,0.25,150$

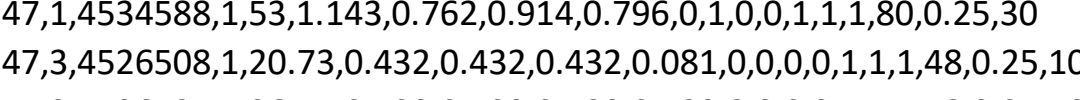
$47,3,4526424,1,26.75,0.533,0.533,0.432,0.123,0,0,0,0,1,1,1,4,48,0.25,10$ (47,1,4526648,1,152, $47,1,4533191,1,1,69,1.143,0.762,0.914,0.796,0,1,0,0,1,1,1,0,0.25,30$ $48,1,4553626,1,79,1.143,0.762,0.014,0.0796,0,1,0,0,1,1,1,1,80,0.25,30$ $48,1,4570929,1,95,1.143,0.762,0.914,0.796,0,1,0,0,1,1,1,1,80,0.25,30$
$48,1,4526855,1,48,1.143,0.762,0.914,0.796,0,0,0,0,1,1,180,0.25,30$ $48,1,4526855,1,48,1.143,0.762,0.014,0.796,0,1,0,0,1,1,1,80,0.25,30$ $48,1,4567812,2,0,59,1.016,0.279,0.406,0.0115,0,0,1,0,0,1,1,1,1,80,0.25,150$ $48,3,4577148,1,7.26,0.33,0.33,0.33,0.036,0,0,0,0,1,1,1,48,0.25,10$ $48,1,4531178,1,59,1.143,0.762,0.914,0.796,0,1,0,0,1,1,1,1,80,0.25,30$

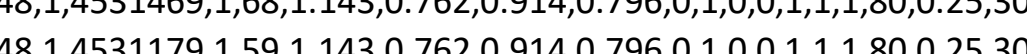
$48,1,4567641,0,89,0.61,0.457,0.457,0.127,0,1,0,0,0,1,1,0,080,0.25,150$ $48,1,4567643,0,067,0.61,0.457,0.457,0.127,0,1,0,0,1,1,0,80,0.25,150$ $48,1,4551799,1,97,0.584,0.381,0.0559,0.124,0,1,1,1,0,1,1,1,51,0.25,30$ $48,1,4531270,1,1,0,1.144,0.062,0.094,0.2496,0,1,1,0,0,1,1,1,180,0.25,30$ $48,1,4531181,1,50,1.143,0.762,0.914,0.796,0,1,0,0,1,1,180,0.25,30$ $48,1,4563667,1,97,0.584,0.381,0.559,0.124,0,1,1,0,1,1,1,51,0.25,30$ $48,1,4526264,0,91,2.997,0.559,0.432,0.724,0,0,2,0,0,0,1,0.5,8,0,0.25,150$ $48,1,4531442,1,58,1.14,0.072,0.014,0.796,0,1,1,0,0,1,1,1,80,0.25,30$ $48,1,4531182,1,50,1.143,0.762,0.914,0.796,0,1,0,0,1,1,80,025,30$ $48,1,4551881,1,59,0.584,0.381,0.559,0.124,0,1,1,0,1,1,1,51,0.25,30$ $48,1,45345855,1,53,1.143,0.762,0.914,0.796,0,1,0,0,0,1,1,1,80,0.25,30$ $48,1,4553520,1,54,1,143,0.762,0.914,0,076,0,1,0,0,1,1,180,0.25,30$ $48,1,4567541,1,25.4,0.584,0.381,0.559,0.0124,0,1,1,0,1,1,1,51,0.25,30$ $48,1,4526139,0,571,5.944,0.635,0.0559,2.11,0,1,1,1,0,1,1,80,0.25,150$ $48,1,4534587,1,51,1.143,0.762,0.914,0.796,0,1,0,0,1,1,1,80,0.25,30$

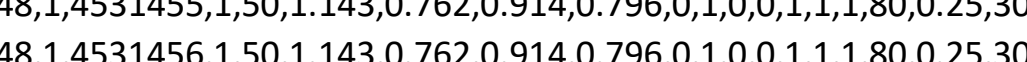

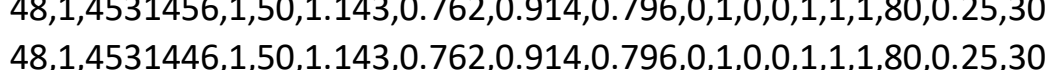
$48,1,4531452,1,50,1.143,0.762,0.914,0.796,0,1,0,0,1,1,1,0,0.25,30$ $48,1,4446664,0,731,6.452,0.635,0.457,1.872,0,1,0,0,0,1,1,80,0.25,150$ $48,3,4577255,1,10.34,0.432,0.432,0.203,0.038,0,0,0,0,0,1,1,1,51,0.25,10$ $48,14531459,150,143,0.762,0914,0.7960,0,0,0,1,1,1,0,025,30$ $48,1,4534599,1,50,1.143,0.762,0.914,0.796,0,1,0,0,1,1,1,80,0.25,30$ $48,1,4563627,1,106,1.143,1.143,0.965,1.261,0,1,0,0,1,1,1,51,0.25,30$ $48,1,4387969,0,0329,2.972,1.219,0.66,2.2991,0,1,1,0,1,1,1,1,00,0.25,150$

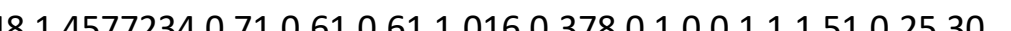
$48,1,4567754,0,63,0.635,0.635,0.813,0.328,0,1,0,0,1,1,1,80,0.25,150$ $48,3,4577166,0,17.9,0.381,0.559,0.813,0.173,0,0,0,0,0,1,1,1,51,0.25,10$ $48,3,4563905,1,16.03,0.028,0.28,0.28,0.022,0,0,0,0,0,1,1,1,48,0.25,10$ $48,144738620,106342930.711,1067,3257,01,1,1,1,1,5,50,025,150$ $48,1,4546758,172,1.143,0.762,0.965,0.84,0,1,0,0,1,1,1,51,0.25,30$ $48,1,4532043,0,0,100,1.143,0.762,1.194,1.04,0,0,1,0,0,1,1,1,51,0.25,30$ $48,1,4553490,1,55,1.143,0.762,0.965,0.84,0,1,0,0,1,1,1,51,0.25,30$ ${ }_{48,1,1,4567750,1,145,0.061,0.61,0.66,0.0 .246,0,0,1,0,0,1,1,1,1,51,0.25,30}$ $48,1,4563952,0,89,1.143,0.762,0.66,0.575,0,1,0,0,1,1,1,51,0.25,30$ $48,1,4551740,1,73.8,1.143,0.762,0.533,0.464,0,1,0,0,1,1,1,51,0.25,30$ $48,1,4577158,0,0,58,0.61,0.61,0.9655,0.359,0,1,0,0,1,1,1,1,51,0.25,30$ $49,146104350,1661,1431,143,1,4221,058,0,1,0,1,1,1,51,025030$ $49,1,4610483,0,140,1.143,1.143,1.168,1.526,0,1,0,0,1,1,1,51,0.25,30$ $49,1,4610482,1,52,1.143,0.762,0.914,0.796,0,1,0,0,1,1,1,1,80,0.25,30$ $49,1,4610429,0,165,1.143,1.143,1.549,2.2024,0,1,0,0,1,1,1,51,0.25,30$ $49,14610419,1197,611430762053304040,0,1,0,1,1,51,250250$ $49,1,4610430,0,0165,1.143,1.143,1.549,2.024,0,1,0,0,0,1,1,151,0.25,30$ $49,1,4610432,0,165,1.143,1.143,1.549,2.0244,0,1,0,0,0,1,1,1,5,0.0 .25,30$ 4.,

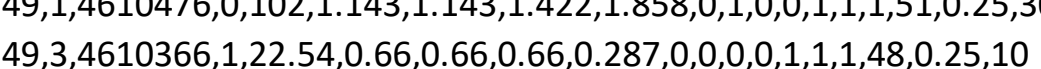
$49,3,4610407,0,8.32,0.533,0.406,0.305,0.066,0,0,0,0,1,1,1,51,0.25,10$ 99,3,4613754,1,2.1, $0.28,0.28,0.28,0.022,0,0,0,0,0,1,1,1,48,0.25,10$ 年, (9., $49,3,4610371,1,15.89,0.635,0.635,0.432,0.174,0,0,0,0,1,1,1,48,025,10$ $49,3,4610458,1,10.87,0.533,0.406,0.406,0.088,0,0,0,0,1,1,1,48,0.25,10$ $49,1,4610478,0,100,1.1433,1.143,1.422,1.858,0,1,0,0,1,1,1,14,0.25,30$ (4), $49,1,4610443,0,166,1.143,1.143,1.575,2.058,0,1,0,0,1,1,51,0.25,30$ $49,1,4610414,0,65.6,0.635,0.635,0.787,0.317,0,1,0,0,1,1,1,1,80,0.25,150$ $49,1,4610408,1,136.4,0.061,0.061,0.06,0.246,0,0,1,0,0,1,1,1,1,0,0.25,30$ 49, $14610418,01231,143,1,143,1,49,1$ $49,1,4610436,0,166,1.143,1.143,1.422,1.858,0,1,0,0,11,1,51,0.25,30$ $49,1,4610481,1,111,1.143,1.143,0.965,1.261,0,1,0,0,1,1,1,151,0.25,30$ $49,4,4615403,0,011.65,1.143,0.152,0.102,0.018,0,0,0,0,0,1,1,1,32,0.125,10$ $49,1,4610409,0,119,1.143,1.143,1.321,1.726,0,0,0,0,0,1,1,1,1,51,0.25,30$ $49,1,4610404,0,116,143,1.143,1321,1726,0,1,0,0,1,1,51,0.25,30$ $49,1,4610485,1,77,1.143,0.762,0.965,0.84,0,1,0,0,1,1,1,51,0.25,30$ $49,1,4610406,0,115,1.143,1.1433,1.321,1.726,0,0,1,0,0,1,1,1,51,0.25,30$ 年, $49,1,4610484,1,122,1.143,0.762,0.914,0.796,0,1,0,0,1,1,1,1,80,0.25,30$ $49,1,4610459,1,237,1.143,0.762,0.965,0.84,0,1,0,0,1,1,1,51,0.25,30$ $49,4,4615444,0,21,1.168,1.168,0.152,0.207,0,0,0,0,0,1,1,1,151,0.25,10$ 年, $49,1,4610491,0,0,3,1.143,1.143,1.041,1.36,0,0,0,0,1,1,1,1,51,0.25,30$
$49,1,4610385,0,113,1.143,1.143,1.321,1.726,0,0,0,0,1,1,1,51,0.25,30$ $49,1,4610479,0,96,1.143,1.143,1.016,1.327,0,1,0,0,1,1,1,151,0.25,30$ $49,1,4610426,0,0,165,1.143,1.1443,1.549,2.2024,0,1,0,0,1,1,1,1,51,0.25,30$ $50,1,4526371,1,146,1.143,1.143,0.965,1.261,0,1,0,0,1,1,1,51,0.25,30$

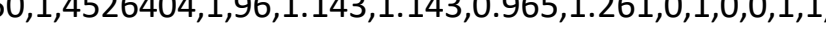
$50,1,4526435,0,0114,1.143,1.143,1.1321,1.726,0,0,0,0,1,1,1,1,51,0.25,30$ $50,1,4526670,1,1,5,1.143,0.762,0.965,0.84,0,1,0,0,1,1,1,1,1,0.25,30$ $50,3,4595464,10860.28028,0280,0220,0,0,1,1,4,0,25,10$ $50,3,4579160,1,0.25,0.18,0.18,0.18,0.006,0,0,0,0,0,1,1,1,48,0.25,10$ $50,1,45877777,1,61.5,1.143,0.762,0.533,0.4644,0,1,0,0,1,1,1,51,0.25,30$ $50,1,4553479,0,113,1.143,1.143,1.321,1.726,0,1,0,0,0,1,1,1,1,01,0.25,30$

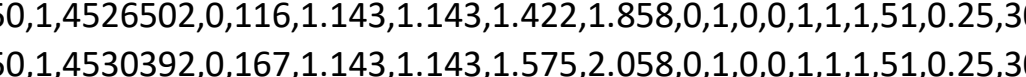
$50,1,4563631,0,116,1.143,1.143,1.422,1.858,0,1,0,0,1,1,1,51,0.25,30$ 
